\title{
Engins de pêche dans les collections du Musée de l'Homme
}

The fishing tools in the Musée de l'Homme (Paris) collections

Serge Bahuchet, Pauline Rameau et Nastassia Reyes

\section{OpenEdition}

Édition électronique

URL : http://journals.openedition.org/ethnoecologie/3533

DOI : 10.4000/ethnoecologie.3533

ISSN : 2267-2419

Éditeur

Laboratoire Eco-anthropologie et Ethnobiologie

Référence électronique

Serge Bahuchet, Pauline Rameau et Nastassia Reyes, " Engins de pêche dans les collections du Musée de l'Homme », Revue d'ethnoécologie [En ligne], 13 | 2018, mis en ligne le 25 juin 2018, consulté le 20 avril 2019. URL : http://journals.openedition.org/ethnoecologie/3533 ; DOI : 10.4000/ ethnoecologie.3533

Ce document a été généré automatiquement le 20 avril 2019.

Revue d'ethnoécologie est mis à disposition selon les termes de la licence Creative Commons Attribution - Pas d'Utilisation Commerciale - Pas de Modification 4.0 International. 


\title{
Engins de pêche dans les collections du Musée de l'Homme
}

\author{
The fishing tools in the Musée de l'Homme (Paris) collections
}

Serge Bahuchet, Pauline Rameau et Nastassia Reyes

\section{Introduction}

1 Nous avions inauguré cette rubrique consacrée aux objets ethnographiques relevant de l'ethnobiologie, des collections du MNHN conservées au Musée de l'Homme, par un premier catalogue des engins de pêche en eau douce d'Afrique centrale, pour accompagner le numéro collectif Inland traditional capture fisheries in the Congo Basin, dirigé par E. Dounias et T. Oishi (Revue d'ethnoécologie 10; Bahuchet \& Rameau 2016). Nous le poursuivons ici avec les engins et accessoires associés à la pêche des autres régions d'Afrique et du Monde.

2 Nous prenons en compte dans ce catalogue les embarcations, en ce qu'elles sont très souvent nécessaires pour réaliser une partie des pêches, et quelques objets associés, après la pêche (préparation des prises) ou liés à la production par élevage (conchyliculture).

3 Nous associons ici deux ensembles administratifs, les objets enregistrés dans la collection de recherche d'ethnobiologie (indexés ETB), et ceux de la collection d'ethnologie (indexés MNHN-E) ${ }^{1}$.

4 Malgré sa diversité et son aspect fragmentaire, notre collection de quelque 80 engins de pêche, près d'une centaine si l'on y ajoute les accessoires annexes liés aux activités halieutiques, permet d'évoquer et d'illustrer l'ensemble des techniques de pêche.

5 Pour les deux-tiers, cette collection est nourrie des missions et séjours de recherche réalisés depuis plus d'une vingtaine d'années, consacrés à l'étude des relations des sociétés humaines avec leur milieu naturel, rendant compte de la diversité des cultures, et notamment de la culture matérielle et des techniques à travers le monde, recherches qui comportent souvent collecte d'objets-témoins. 


\section{Qu'est-ce que la pêche ? Qu'est-ce qu'un poisson?}

6 On désigne par ce terme de "pêche » l'ensemble des pratiques de capture des animaux aquatiques, le terme de «chasse » étant usuellement réservé à la capture des animaux terrestres ou volants. Toutefois la similitude des armes ou ustensiles de capture, entre la chasse, la pêche et même la guerre, a déjà été relevée depuis longtemps (Leroi-Gourhan (1945) 1973); ce qui compte ce n'est pas le type d'animal, mais son mouvement et son milieu de vie.

7 Ainsi, la prise d'un phoque depuis un kayak, avec un harpon, n'est-elle pas différente, du point de vue ethnoécologique, du harponnage d'un requin, d'une baleine, ou d'un hippopotame.

Cette remarque renvoie à la définition de la proie aquatique. Qu'est-ce donc qu'un " poisson »? Le terme usuel de "poisson » désigne tout ce qui vit et se meut dans l'eau, quelle que soit sa morphologie, et son appartenance taxonomique. Si l'on consulte ce qui est considéré comme le tout premier traité d'ichtyologie, L'histoire entière des poissons de Guillaume Rondelet publié en 1558, il y a dedans ce que nous considérons actuellement comme poissons, mais aussi des mammifères (dauphins, phoques...), des reptiles (tortues), des mollusques... (Rondelet 2002). C'est également l'acception du terme dans le grand Traité général des pêches de Henri Duhamel du Monceau, rédigé deux siècles plus tard (1769), et consacré à « l'histoire des poissons ou des animaux qui vivent dans l'eau ».

D'ailleurs dans la classification phylogénétique actuelle, on chercherait en vain quelque chose qui regroupe "les poissons» du sens commun, lesquels sont dispersés dans plusieurs clades, tels que Chondrichthyens, Actinoptérygiens ou Sarcoptérygiens (Lecointre \& Le Guyader 2001 : 531).

\section{Mer, rivière?}

Dans le même ordre d'idée, du point de vue technique, il n'y a pas vraiment nécessité de distinguer pêche en mer ou pêche en eau douce. Certes, pêcher à Paris depuis un pont de la Seine, s'inscrit dans un contexte a priori totalement différent de celui dans lequel se trouve un pêcheur embarqué en mer, mais l'action menée par l'Homme reste assez similaire quelque soient les lieux ou les régions du monde. On trouvera difficilement une différence, dans les gestes et les engins employés, entre un pêcheur européen exerçant en mer et celui pêchant sur un lac ou au milieu d'un fleuve équatorial telle l'Amazone, même à plusieurs milliers de kilomètres de son embouchure (Figure 1). L'acte de pêche se pratique depuis le bord que ce soit d'une rivière, d'un étang, depuis une grève ou depuis une embarcation; on disposera des engins de pêche autant sur l'estran que dans les marais. D'ailleurs la similitude des outils employés de par le monde suscite un constant étonnement sur les adaptations technologiques semblables de tous les hommes. 
Figure 1 : Aspect de l'Amazone à lquitos, Pérou

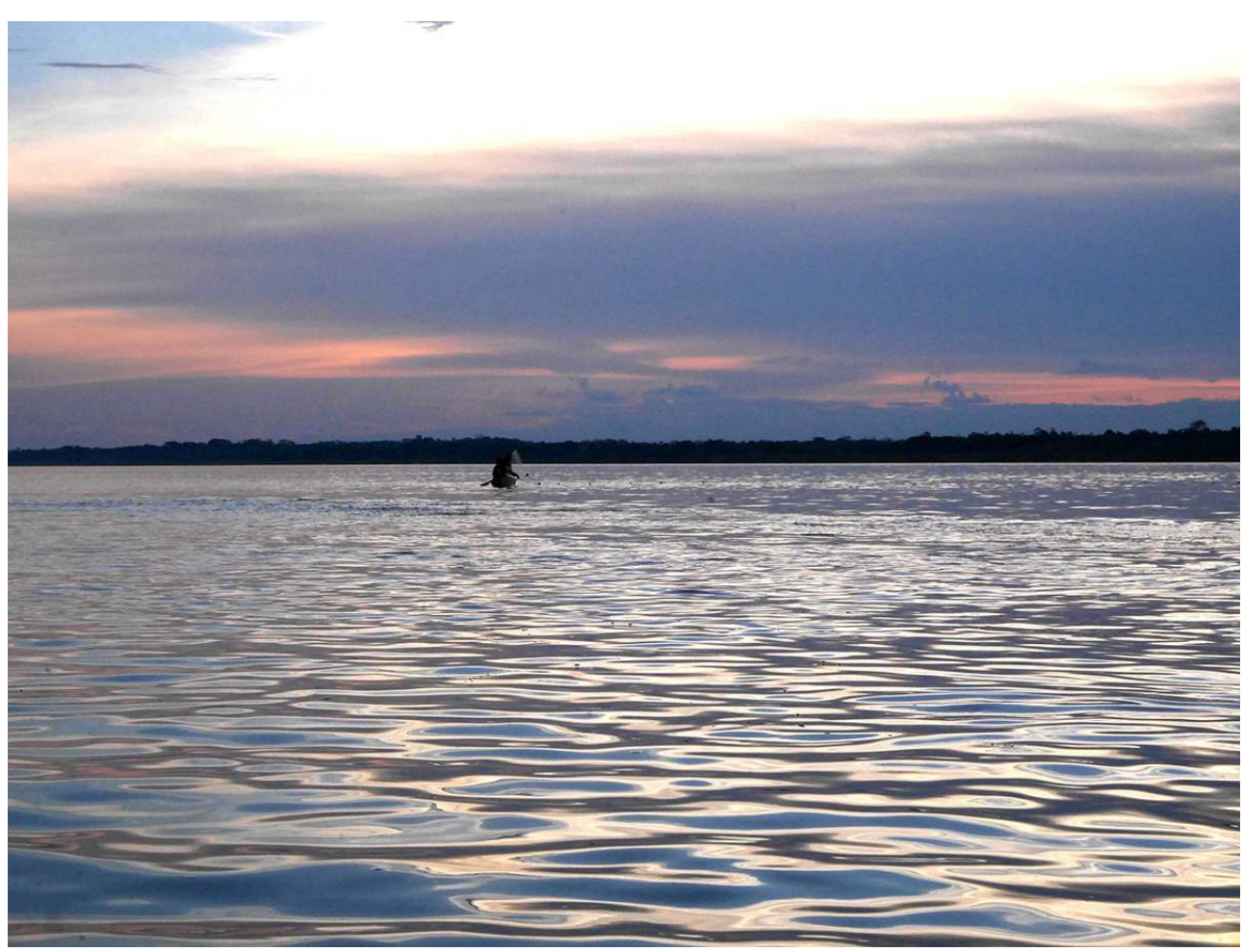

(c) S. Bahuchet

\section{Plan de classement}

11 Pour organiser les éléments de la collection des engins de pêche, nous avons choisi la classification fonctionnelle de Théodore Monod (1973), qui met en avant selon sa judicieuse expression «le point de vue du poisson». Basée sur le comportement de la proie ou l'effet sur elle, et non sur la forme technique de l'engin, elle nous semble relever de critères ethnoécologiques, conformes à notre approche.

12 Monod organise ainsi les engins en fonction de leur place et leur mouvement par rapport au corps du poisson, qui est perforé ou contenu par des parois immobilisantes, lesquelles sont animées ou fixes. Il définit 12 classes, qui se divisent en sous-classes puis en familles, selon une terminologie empruntée à la zoologie (Tableau 1). 
Tableau 1 : Résumé de la classification (Monod 1973 : 225)

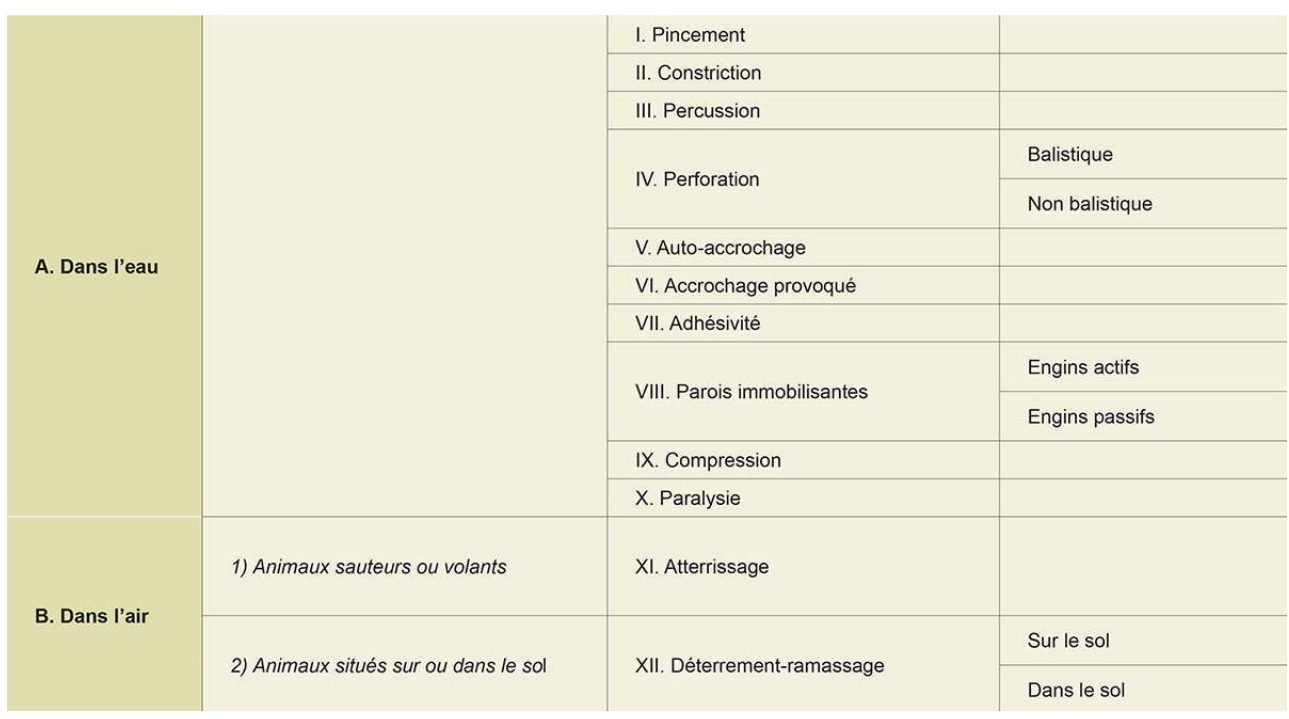

Ces classes ne mettent pas toutes en jeu des engins, aussi ne sont-elles pas toutes présentes dans les collections ethnographiques. Dans notre catalogue, nous faisons état de six classes, dont deux dominent très largement, la IV-Perforation, où l'on distingue la perforation balistique (harpons, flèches) et non balistique (hameçons), et la VIII-Parois immobilisantes, actives (épuisettes, chaluts...) ou passives (filets calés, nasses...).

La collection recensée ici compte 77 engins de pêche (objets individuels ou lots), qui sont inclus dans cinq des classes proposées par Théodore Monod :

IV- Perforation : 39

V- Auto-accrochage : 1

VI- Accrochage provoqué $: 1$

VIII- Parois immobilisantes : 32

X-Paralysie : 2

XII- Déterrement-ramassage : 2 .

On peut comparer cette répartition des techniques avec le matériel de pêche d'Afrique centrale (Bahuchet \& Rameau 2016). Nous avons présenté, dans ce précédent article, une collection de 22 objets, dont les deux-tiers sont d'ailleurs des vanneries : 6 de la classe IVPerforation, 8 de la classe VIII-Parois immobilisantes, mais 8 de la classe XII-Déterrementramassage, ce qui dans ce cas précis correspond aux techniques d'écopage, qui pourraient bien apparaître, avec les nasses incluses dans la classe VIII, comme une particularité de l'Afrique centrale.

Ce petit exercice de comparaison permet d'attirer l'attention sur certaines techniques, qui mobilisent une culture matérielle spécifique.

Les objets réunis dans les collections, rapportés de différentes régions du monde, ne forment qu'une partie des activités de pêche, un reflet; en effet, certaines activités halieutiques n'impliquent pas l'usage d'objets, et d'autres engagent des engins totalement intransportables - ainsi il y a une part circonstancielle non négligeable dans le fait que le chercheur rencontre et rapporte du terrain un engin plutôt qu'un autre. Aussi nos collections ne prétendent-elles pas à une exhaustivité des pratiques pour chaque communauté visitée. Mais elles permettent de mettre en regard des objets qui présentent des similitudes, parfois très étonnantes, et des différences. Ces contrastes témoignent de 
la richesse des cultures humaines, et suscitent des interrogations des plus captivantes pour anthropologues et ethnoécologues.

18 L'objet lié à une technique de capture permet plusieurs niveaux de lecture. En premier lieu, on peut observer et analyser sa forme et son fonctionnement mécanique, dans la réserve du musée, hors du contexte du terrain; c'est l'enquête de technologie culturelle. L'enquête sur place situe l'objet dans son contexte écologique (à quel endroit est-il placé dans le milieu aquatique ?) et précise son mécanisme éthologique (quel comportement de la proie met-il en action?). En cela, il relève d'une approche ethnoécologique.

\section{Le catalogue}

Chaque objet est présenté sous forme d'une fiche, accompagnée d'une photographie, comportant les éléments suivants :

Nom de l'objet

Nom de l'ethnie. Pays ; village, région (département)

Description, usage, matières

Date de collecte ; nom du collecteur

Dimensions en centimètres

Numéro d'inventaire : dans la collection d'ethnobiologie (ETB-code ISO pays-initiales du collecteur-année-n ${ }^{\circ}$ objet); dans la collection d'ethnologie (MNHN-E-année.n ${ }^{\circ}$ collection. $n^{\circ}$ objet).

\section{L'embarcation}

\section{1) Canot en roseaux des côtes du Pérou}

Figure 2: Canot en roseaux des côtes du Pérou MNHN-E-2010.1.1

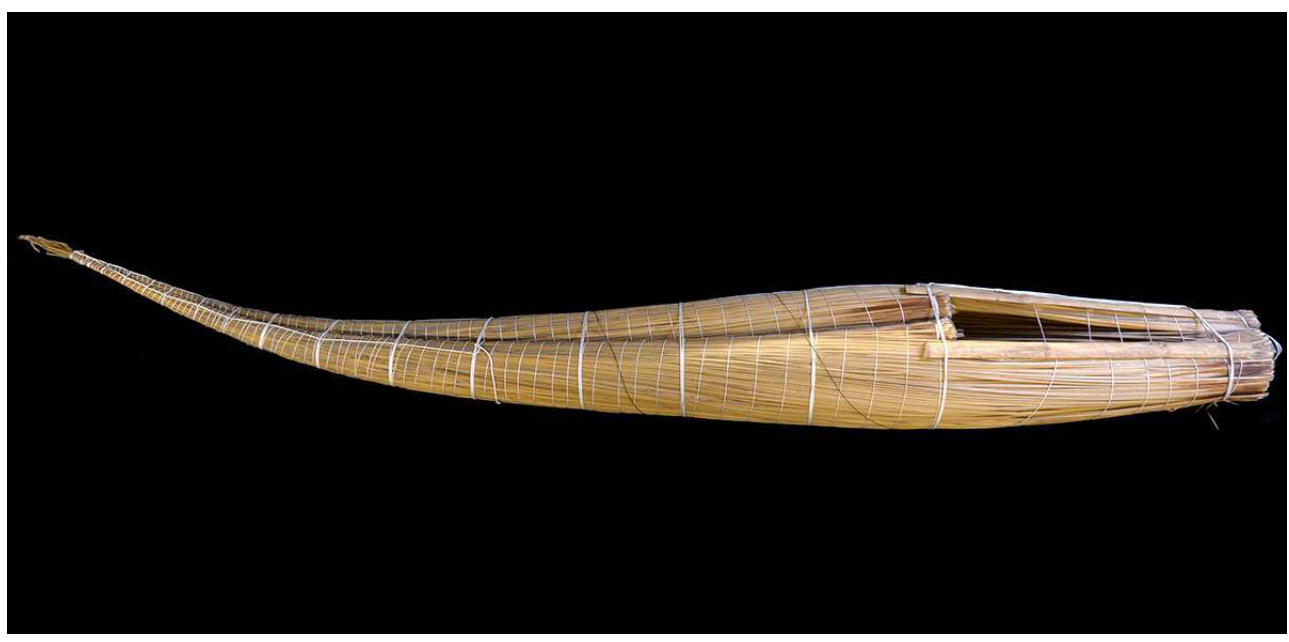

(c) J.-C. Domenech

- Pérou, Huanchaco, département La Libertad; province Trujillo

- Caballito de totora. Il est fait de tiges de totora (Schoenoplectus californicus, Cypéracées). C'est un bateau de mer individuel fait en roseau, destiné à la pratique d'une pêche artisanale traditionnelle et millénaire. Sa légèreté lui permet de franchir la barre. La forme des barques côtières est différente de celle des barques de roseau fabriquées sur le 
lac Titicaca. Un caballito est composé de deux faisceaux de cannes en forme de fuseau, attachés l'un à l'autre, chacun étant lui-même composé de deux parties enchâssées l'une dans l'autre, la mère madre (à l'extérieur) et le fils hijo, à l'intérieur (Figures 3, 6). Ainsi est défini une sorte d'habitacle (la « boîte » caja) dans laquelle le pêcheur s'agenouille. De nos jours, toujours utilisée pour la pêche, cette embarcation trouve une nouvelle vie avec la pratique du surf sportif. Pour l'alléger et diminuer la quantité de tiges nécessaires, la barque est souvent remplie de formes en mousse ou bien de bouteilles de plastique vides (Figure 5)

-26/08/2009; collectrice Marina Quiñe

- Longueur $472 \mathrm{~cm}$, largeur $65 \mathrm{~cm}$, hauteur $50 \mathrm{~cm}$

- MNHN-E-2010.1.1 (exposé dans la Galerie de l'Homme)

- Références : Banack et al. 2004, Quiñe 2009, Rondón et al. 2003

Figure 3 : Phases de la construction du CABALLITO
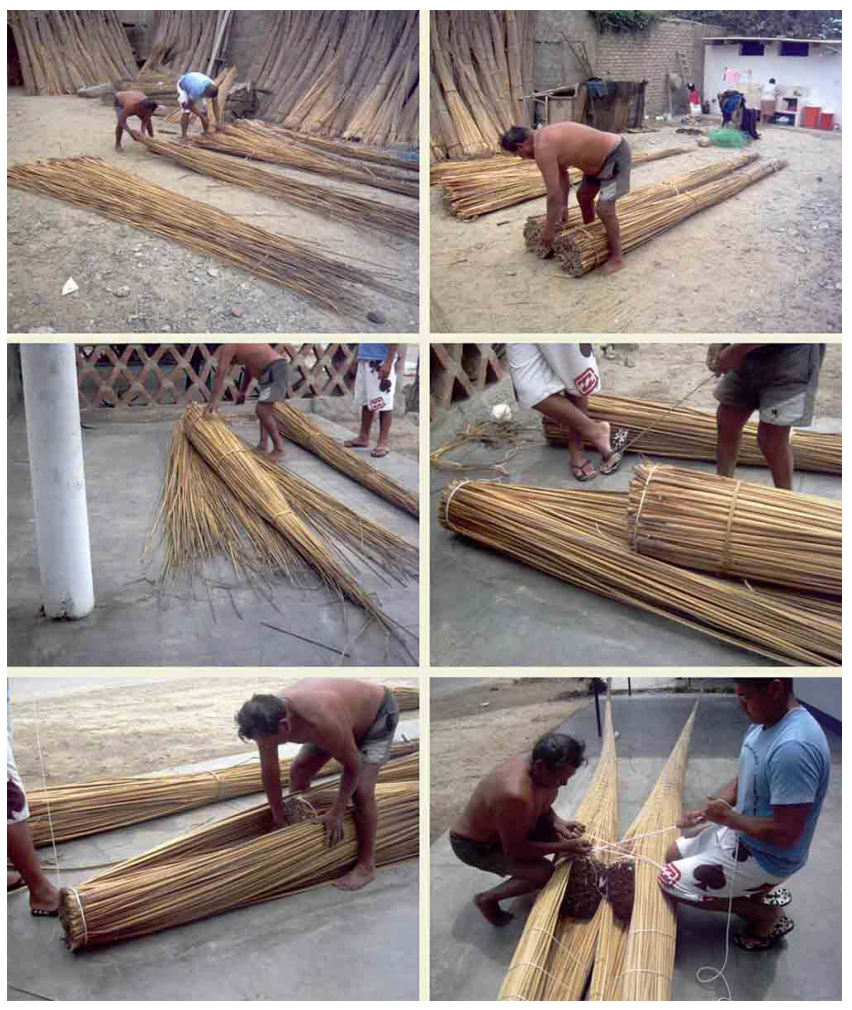

(c) M. Quiñe 
Figure 4 : Les CABALLITOS séchant sur la grève, après la sortie de pêche

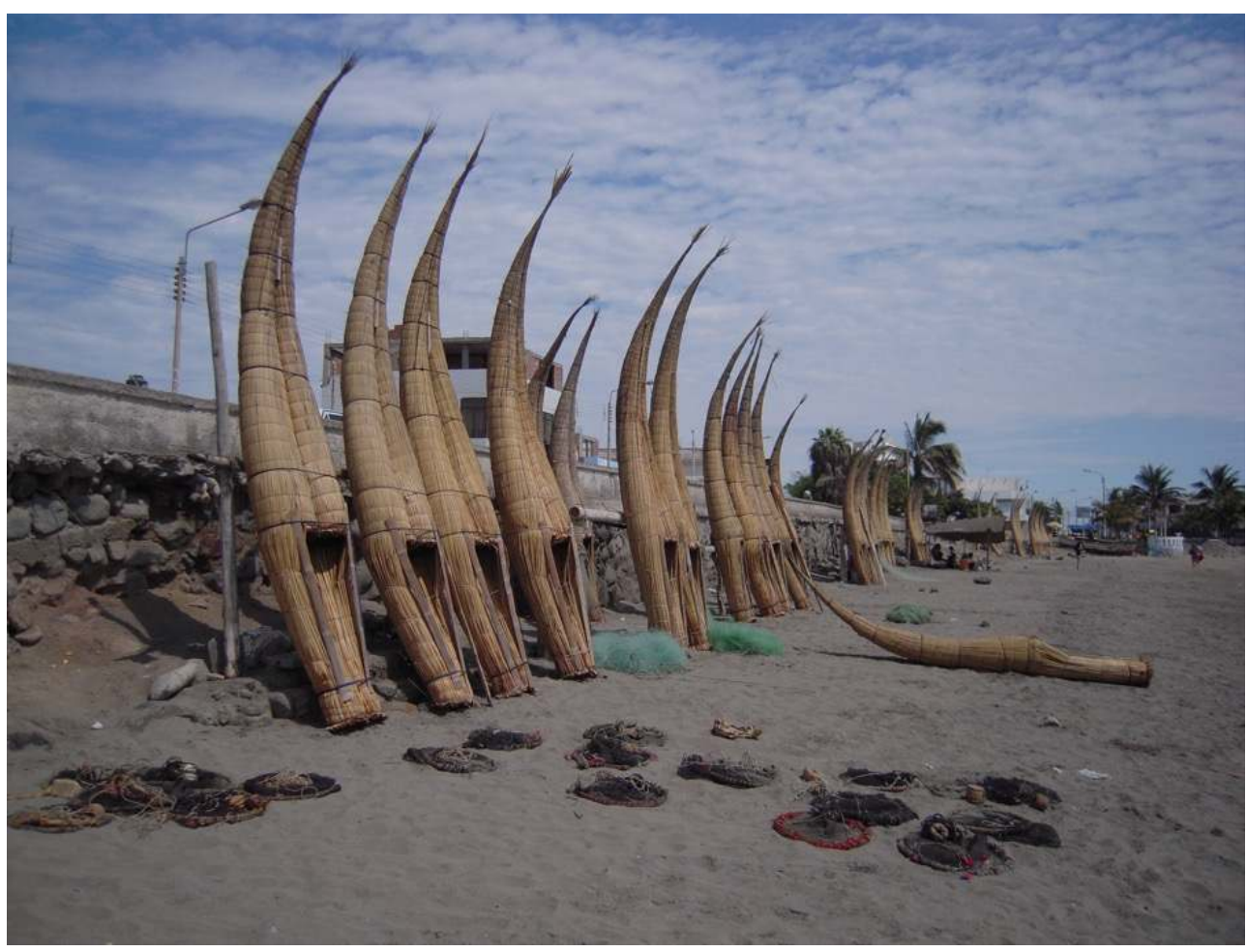

(c) M. Quiñe

Figure 5 : Un CABALLITO « moderne », rempli de flotteurs de polystyrène

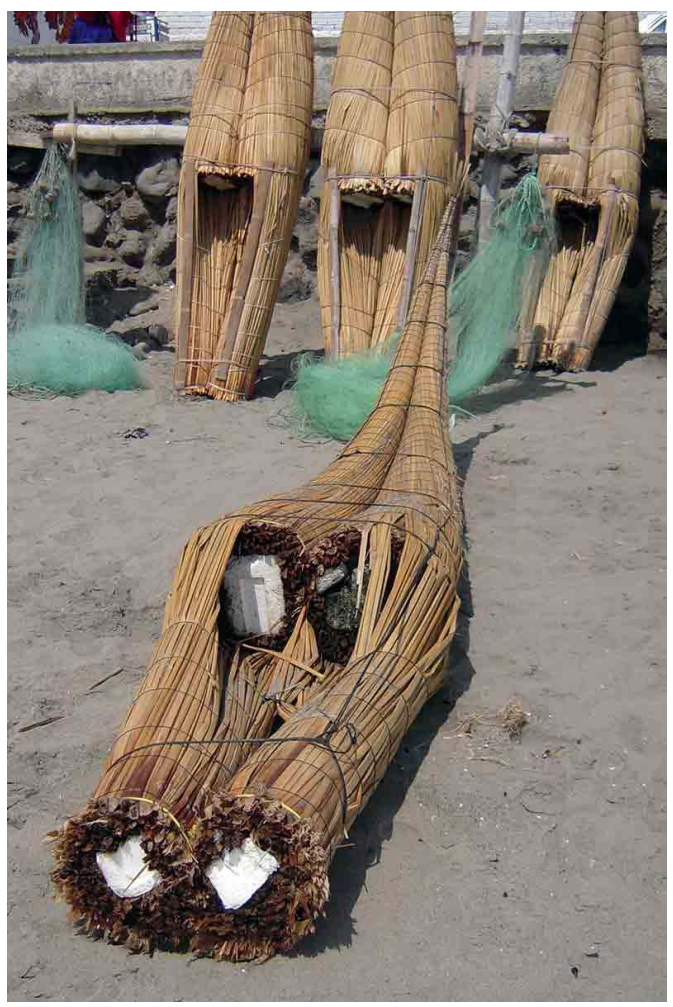

(c) M. Quiñe 
Figure 6 : Les parties du CABALLITO DE TOTORA

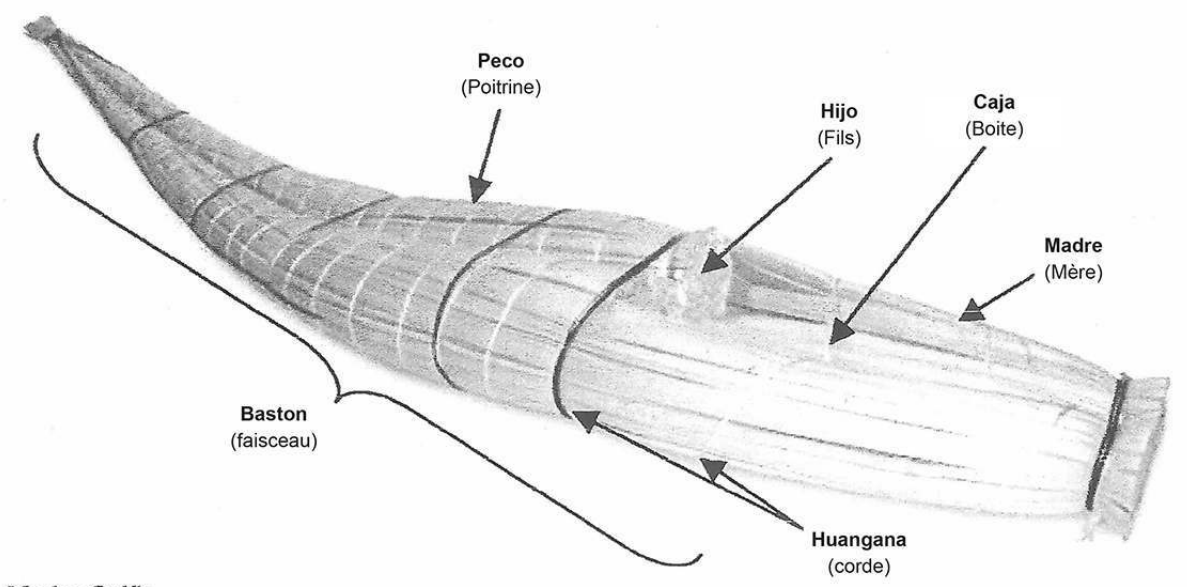

Marina Ouiñe

D’après Quiñe 2009 : 32

\section{2) Maquette de Caballito de totora}

Figure 7 : Maquette de CABALLITO DE TOTORA ETB-PE-MQ-2009-008

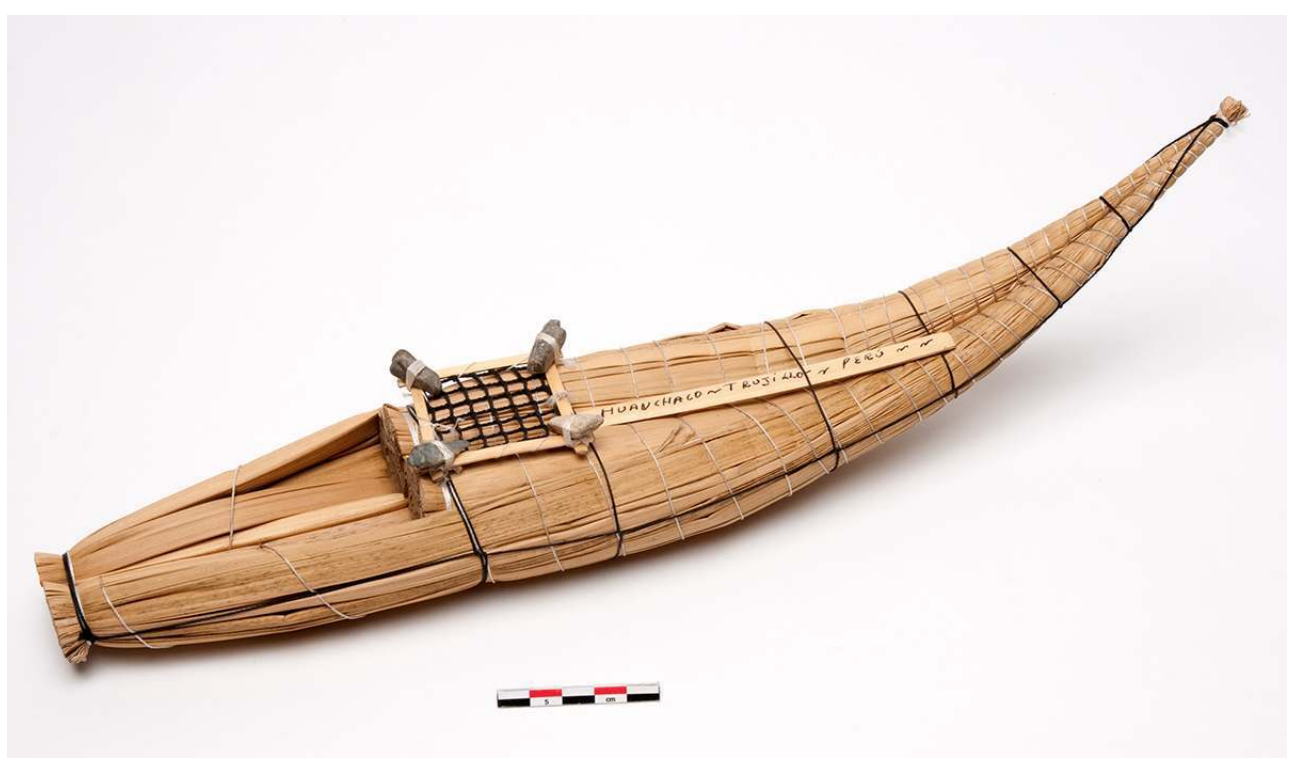

(C) J.-C. Domenech

23 - Pérou, Huanchaco, département La Libertad ; province Trujillo

- Modèle réduit de Caballito de totora. Il est fait de la même façon qu'un vrai, en utilisant comme matière première la totora (Schoenoplectus californicus, Cypéracées). Le Caballito de totora est un bateau individuel fait en roseau, destiné à une pêche artisanale traditionnelle et millénaire. Élément de décoration qui représente un des objets phare de la culture locale

-26/08/2009 ; collectrice Marina Quiñe

- $40 \times 10 \mathrm{~cm}$

- ETB-PE-MQ-2009-008 
Figure 8 : Réplique d'une céramique Chimu ETB-PE-MQ-2009-023

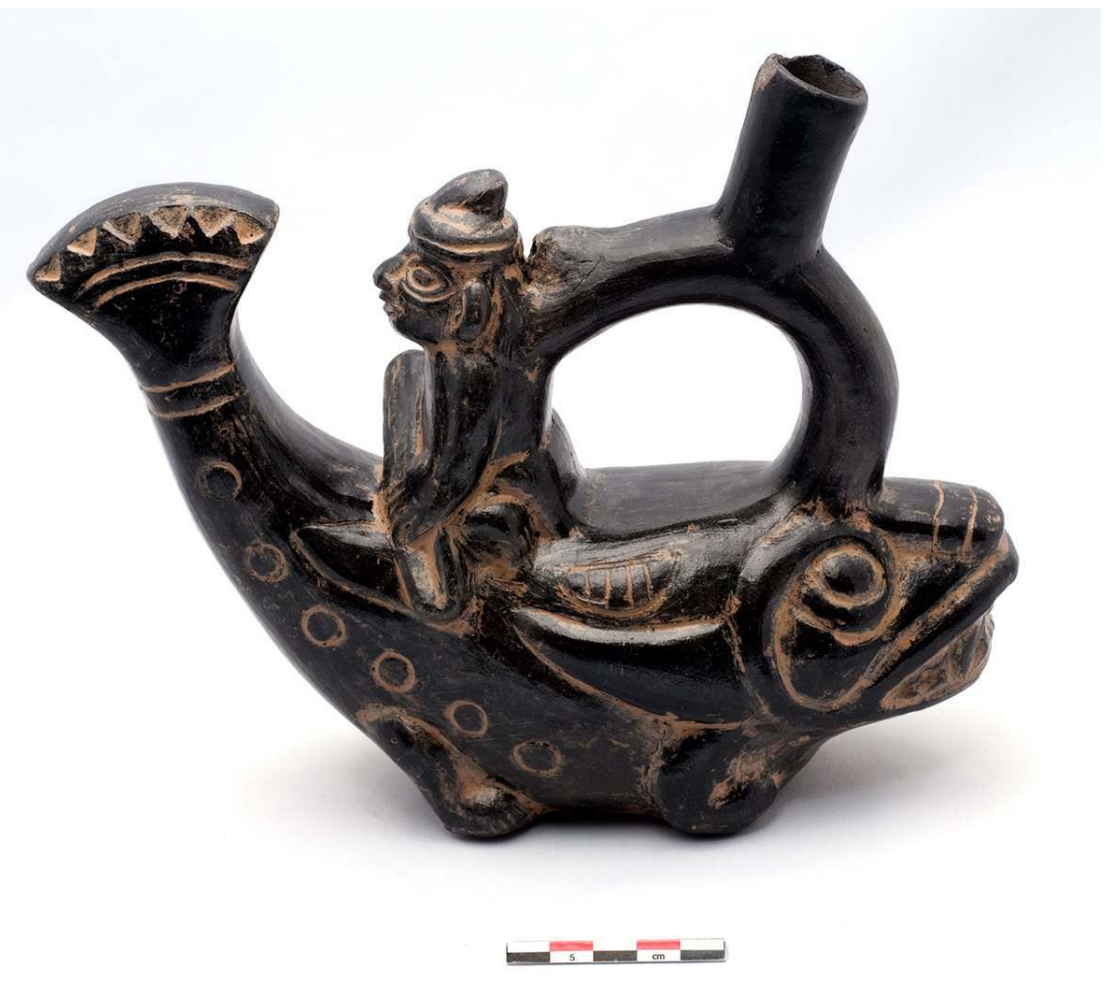

\section{(C) J.-C. Domenech}

- Pérou, département La Libertad ; province Trujillo

- Réplique d'une pièce de céramique de la culture Chimu (XI - XV siècle, nord du Pérou), représentant un pêcheur sur un caballito de totora en forme de poisson géant Réplique destinée à la décoration ou pour des amateurs de céramique

-23/06/2009 ; collectrice Marina Quiñe

- Longueur $25 \mathrm{~cm}$, hauteur $15 \mathrm{~cm}$

- ETB-PE-MQ-2009-023 (exposé dans la Galerie de l'Homme)

4) Pirogue monoxyle du Vanuatu MNHN-E-2012.8.1

[pas de photo disponible de cet objet]

- Vanuatu, village de Lalé, îles Vanikoro

- Pirogue de mer en bois, de type monoxyle, avec deux planches transversales servant de sièges. Manœuvrée à l'aide d'une pagaie, elle servait aux déplacements intervillageois et à la pêche côtière à l'intérieur du lagon. Forme typique des embarcations mélanésiennes à l'Ouest des Iles Vanikoro

- 2008 ; collecteur Yves Gladu ; don SAMH

- Longueur $390 \mathrm{~cm}$, largeur $50 \mathrm{~cm}$

- MNHN-E-2012.8.1

5) Modèle de pirogue du Venezuela 


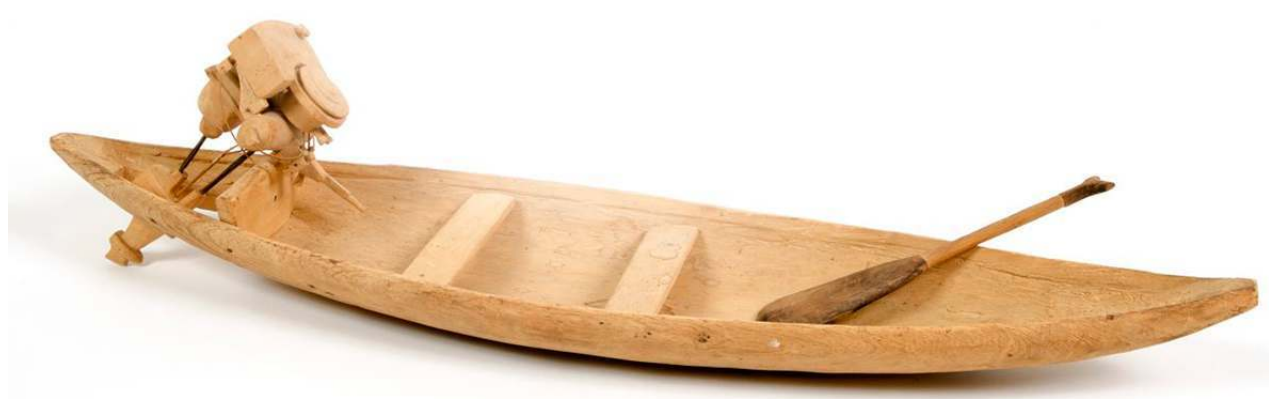

(c) J.-C. DOMENECH

- Ethnie Warao. Venezuela, Delta de l'Orénoque

- Modèle de pirogue en bois tendre avec son moteur, deux banquettes et une pagaie

- 1974 ; collectrice Brigitte Gladu

- Longueur $66 \mathrm{~cm}$, largeur $16 \mathrm{~cm}$, hauteur $10 \mathrm{~cm}$

- MNHN-E-2012.7.6

\section{6) Modèle de Kayak du Groenland}

Figure 10 : Modèle de Kayak du Groenland MNHN-E-2016.6.87

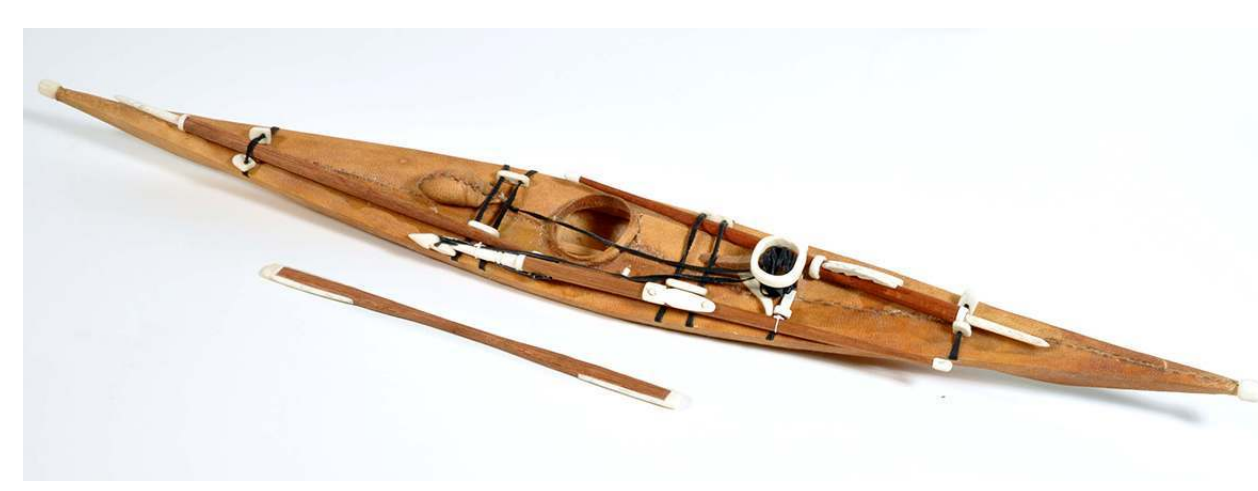

(c) D. Brabant

- Inuit. Groenland, côte ouest. Acquis pour des activités et démonstrations didactiques et éducatives

- Ce modèle réduit de kayak en peau de phoque comporte sur le pont, arrimés, trois projectiles différents, en bois et en os : une pique droite atsingaq (pour les phoques gras, en automne), une lance à trois pointes nukkit, destinée à la capture des oiseaux posés sur l'eau, et enfin un harpon saaqqit dont la pointe amovible est reliée à un flotteur puttarit par un filin de cuir atsinaaq. Celui-ci est soigneusement lové dans un support ovale, devant le chasseur (Robbe 1994 : 252-254). Ce harpon est l'arme employée pour la chasse aux phoques (Figure 11). De nos jours, seul le harpon est encore utilisé, en même temps que le fusil.

- 1988 ; collecteur Didier Dechery, don Pierre et Bernadette Robbe 
- Longueur $54,5 \mathrm{~cm}$, largeur $6 \mathrm{~cm}$, hauteur $3,5 \mathrm{~cm}$

• MNHN-E-2016.6.87

Figure 11 : Lancement de harpon avec le propulseur, pour la chasse au phoque, depuis le kayak

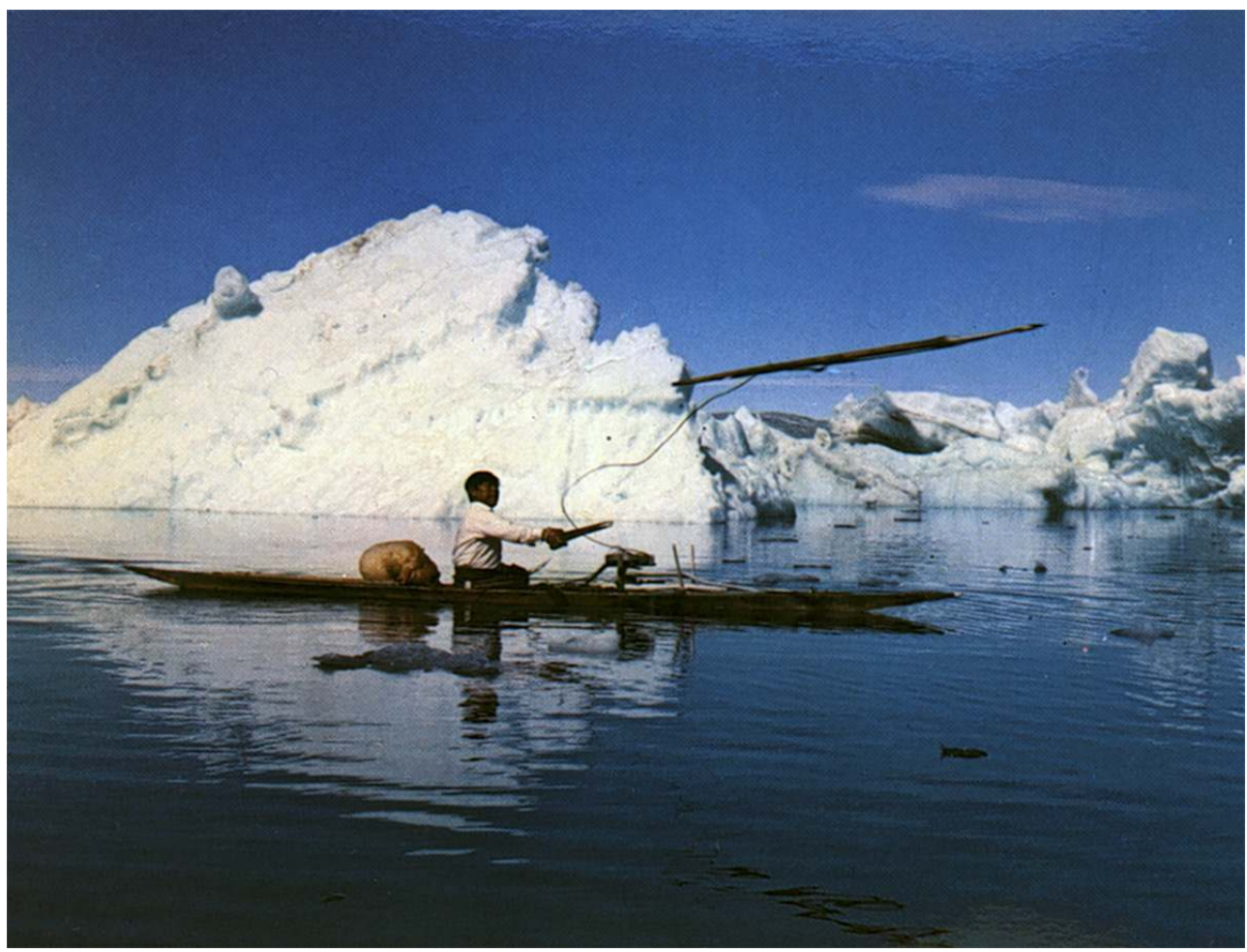

On distingue le support du filin du harpon devant le chasseur, et le flotteur derrière lui. Ammassalik, 1969

(C) P. Robbe

33 Manœuvre de l'embarcation : les pagaies

34 7) Pagaie du Surinam 
Figure 12 : Pagaie du Surinam ETB-SR-MCC-2003-032

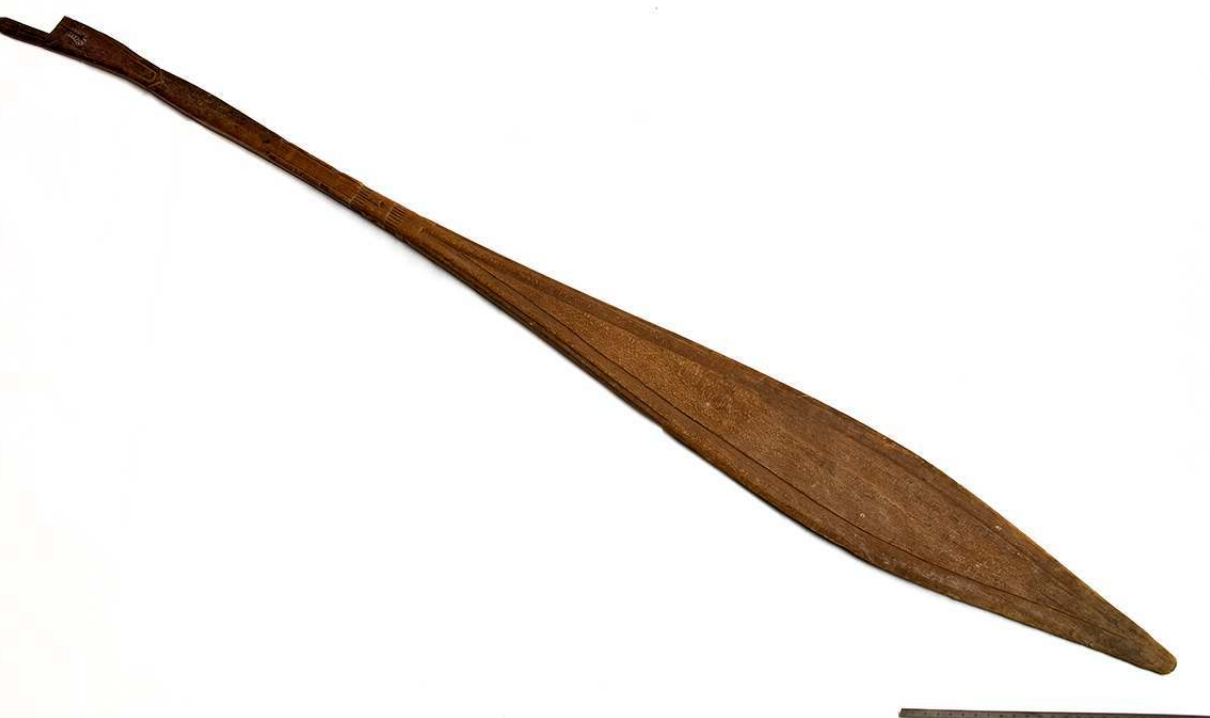

\section{(C) J.-C. Domenech}

- Saramaka. Surinam, village Maipà, rivière Suriname

- Probablement en bois d'acajou amer Cedrela odorata (Meliacées)

- Avril 2003 ; collectrice Monica Castro Carreño

- Longueur $135 \mathrm{~cm}$, largeur $11 \mathrm{~cm}$

- ETB-SR-MCC-2003-032

36 8) Pagaie de Papouasie-Nouvelle Guinée 
Figure 13: Pagaie de Papouasie-Nouvelle Guinée MNHN-E-2006.1.35

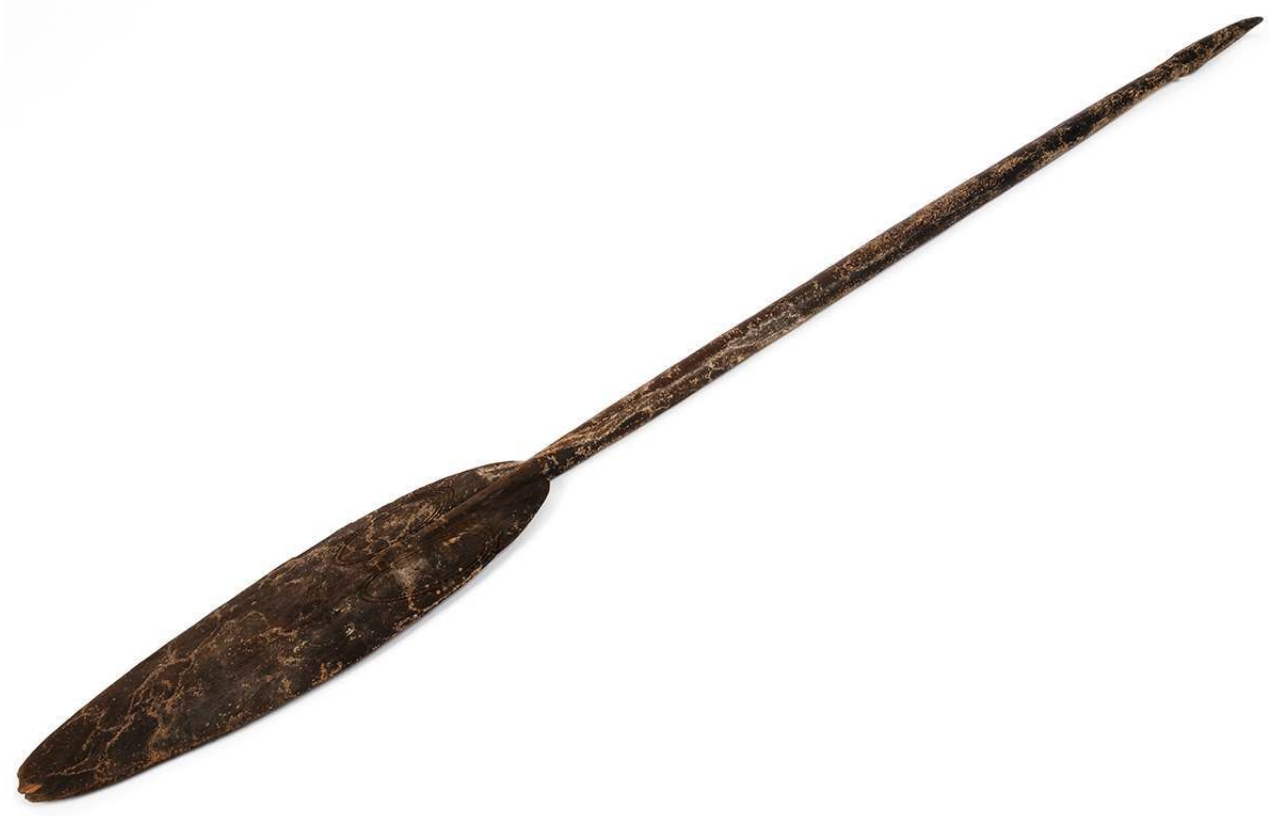

\section{() J.-C. Domenech}

37 - Papouasie-Nouvelle-Guinée, Lac Murik, Sepik

- Cette pagaie est gravée sur la partie inférieure de la pelle de deux séries d'ellipses figurant les yeux ; le nez en relief est constitué par le prolongement d'un long manche dont l'extrémité est sculptée en forme de tête d'oiseau

- Collecteur Christian Coiffier

- Longueur 138,5 cm, largeur $15 \mathrm{~cm}$

- MNHN-E-2006.1.35

9) Deux pagaies de l'Archipel Santa Cruz 


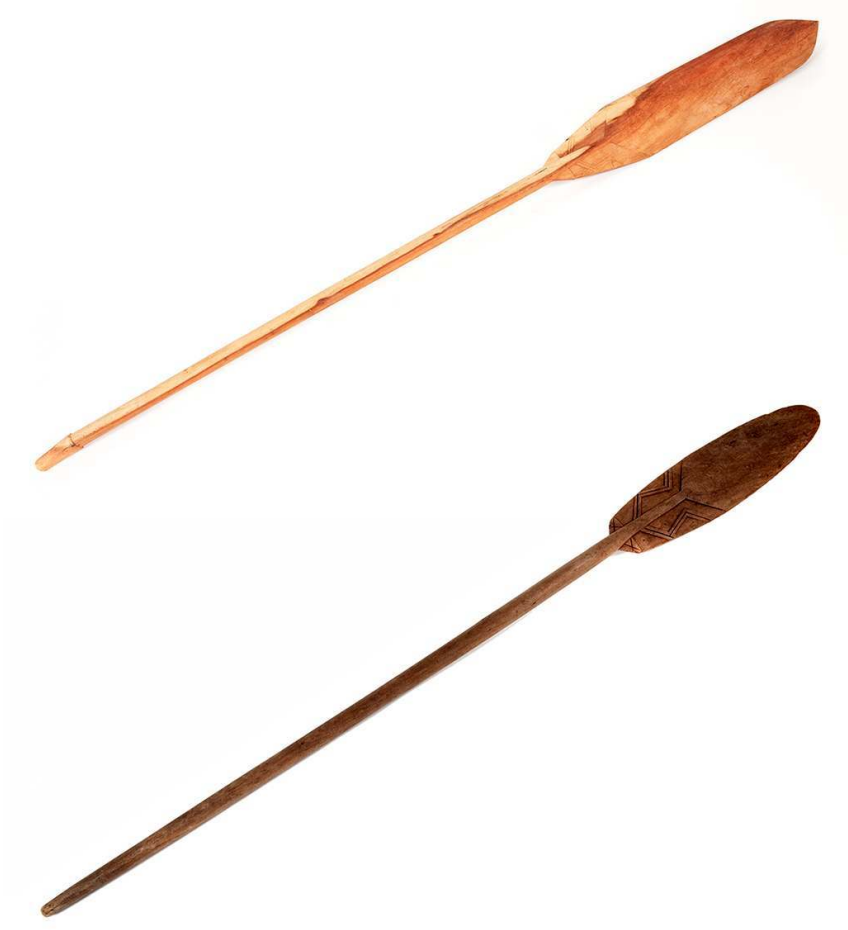

(c) J.-C. Domenech

39 - Îles Salomon, Archipel Santa Cruz.

- 2009.15.6: décor constitué de deux double-zigzags en relief et de deux croix, symétriques, sculptés depuis la base. 2009.15.7 : décoré de deux double-zigzags en relief, symétriques, sculptés depuis la base

- 2008 ; collecteur Christian Coiffier

- 2009.15.6 : longueur totale $151 \mathrm{~cm}$, largeur de la pale $13 \mathrm{~cm} ; 2009.15 .7$ : longueur totale $143 \mathrm{~cm}$, largeur de la pale $12 \mathrm{~cm}$

- MNHN-E-2009.15.6 et 2009.15.7

10) Modèle réduit de pagaie du Pérou 
Figure 15 : Modèle de pagaie du Pérou ETB-PR-ERO-2017-009

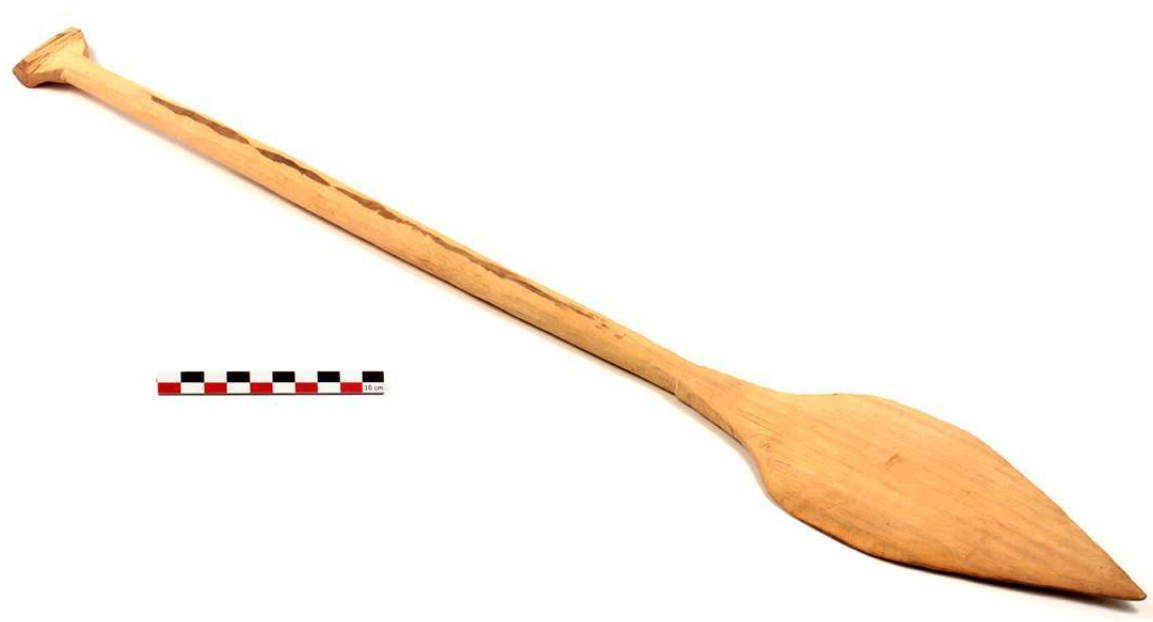

(c) P. Rameau

41

- Ethnie Maijuna. Pérou, communauté de Nueva Vida, rivière Yanayacu, région Loreto, province Maynas

- Modèle réduit de pagaie, fait par Neida Mosoline Peterman. Nom de l'objet yórì. Nom de l'arbre remo caspi (probablement Aspidosperma sp., Apocynacées)

- 14 août 2017 ; collectrice Emmanuelle Ricaud Oneto

- Longueur $67 \mathrm{~cm}$, largeur $10 \mathrm{~cm}$ (taille réelle $1,20 \mathrm{~m}$ )

- ETB-PR-ERO-2017-009 
Figure 16 : La pagaie en usage, de retour du jardin

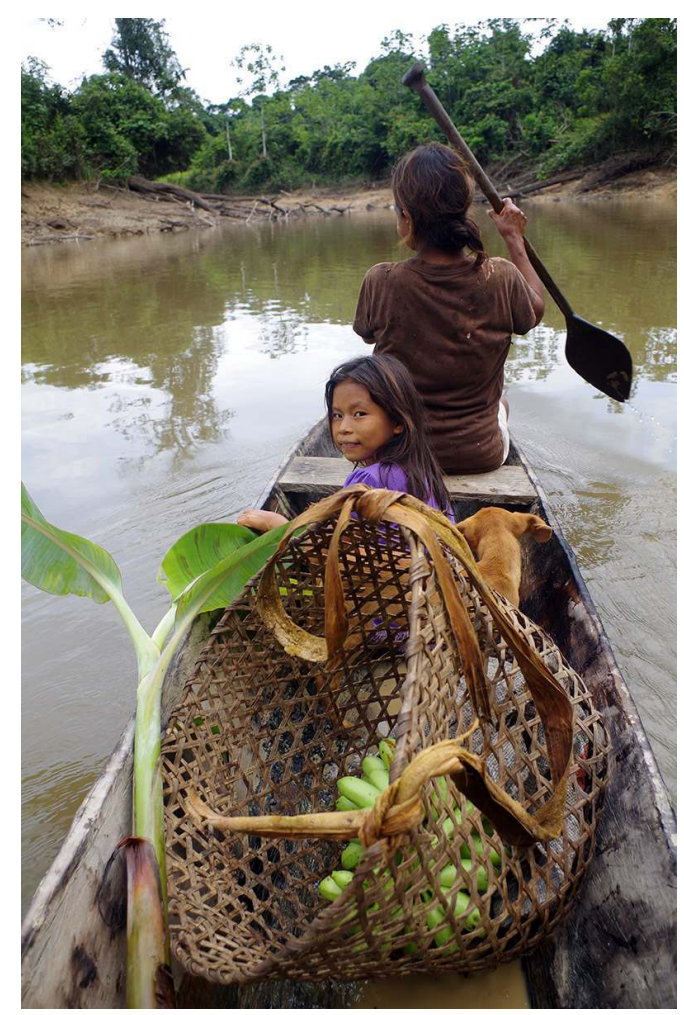

(c) E. Ricaud Oneto

42 11) Deux modèles de pagaie d'Indonésie 
Figure 17 : Deux modèles réduits de pagaie d'Indonésie ETB-ID-ALB-2013-001 et 002

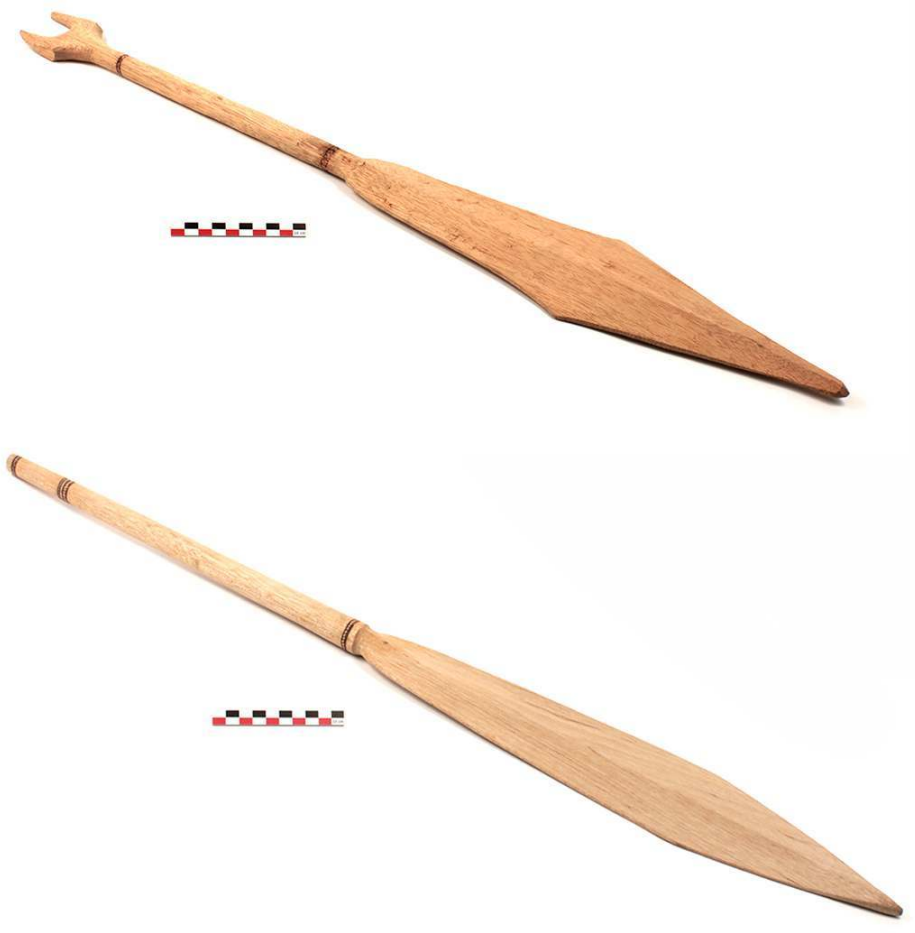

(c) P. Rameau

- Ethnie Mentawai. Indonésie, Sarausau, île de Siberut

- Les deux rames s'appellent luga. Fabriquées par Geremias Sabuku. Hommes et femmes manient les pirogues, notamment pour la recherche quotidienne des mollusques dans la mangrove (Burgos 2013)

- 2013 ; collectrice Ariadna L. Burgos

- $001: 78 \times 11 \mathrm{~cm} ; 002: 88 \times 7,5 \mathrm{~cm}$

- ETB-ID-ALB-2013-001, 002

Figure 18 : Les pagaies des Mentawai
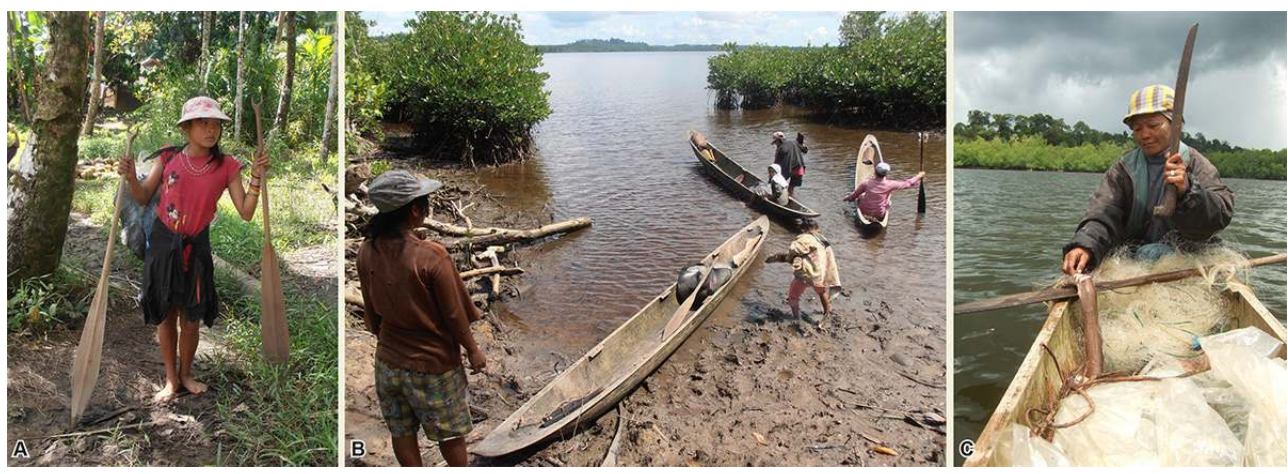

A) Rita attend sa sœur pour partir à la pêche dans la baie de Katurai (2014). B) La pagaie est ancrée dans la vase de la mangrove de Katurai afin de stabiliser et figer le mouvement du bateau (2011). C) Se servant de sa pagaie comme billot, Ina Lucia essaie de tuer un « poisson-serpent » maléfique qui s'est pris dans son filet. Elle ne s'en est débarrassée qu'après une longue lutte et n'en a gardé aucun morceau (Baie de Katurai, 2011)

(c) A. Burgos 
12) Étendards de pêcheurs du Japon

Figure 19 : Étendards de pêcheurs ETB-JP-NC-2013-01, 02, 03, 04

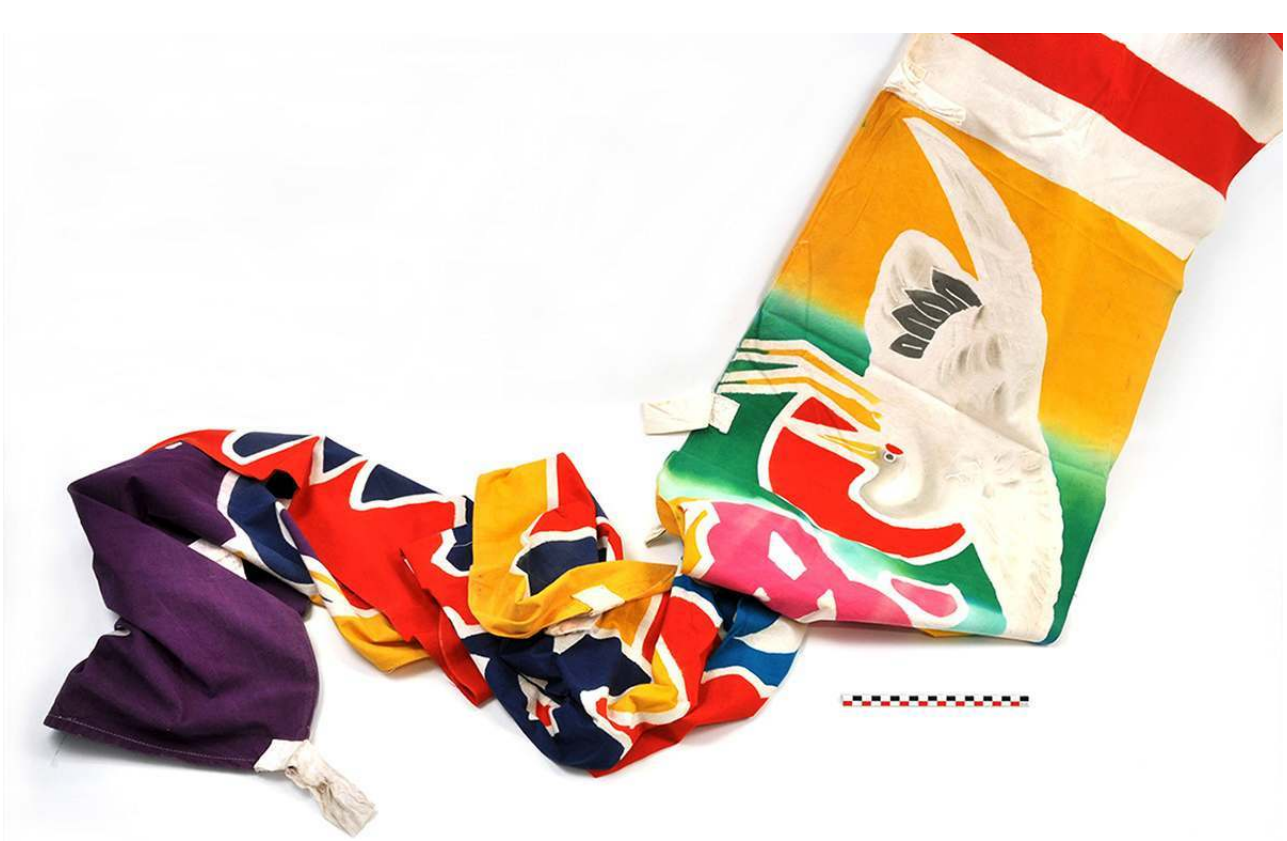

A

(C) P. Rameau

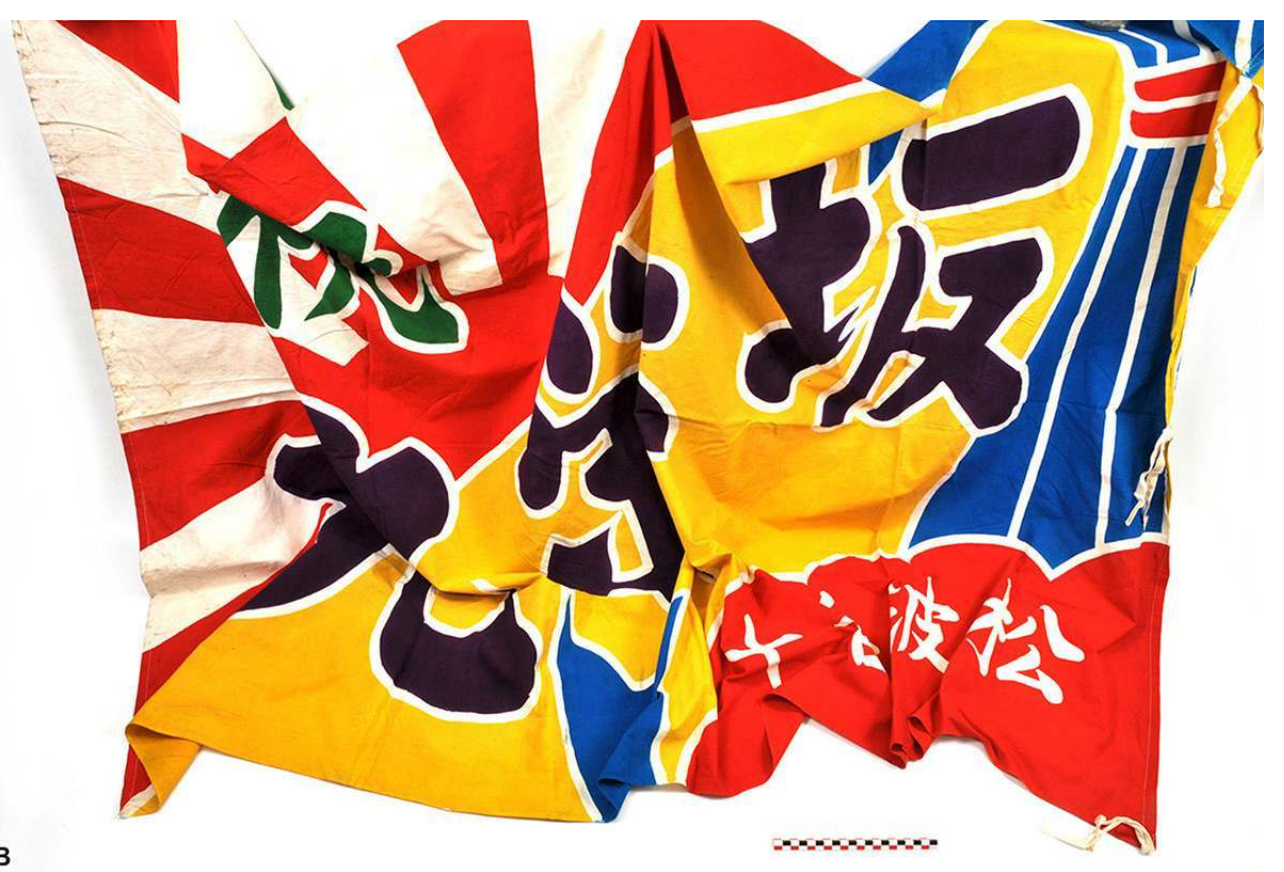

(C) P. Rameau 


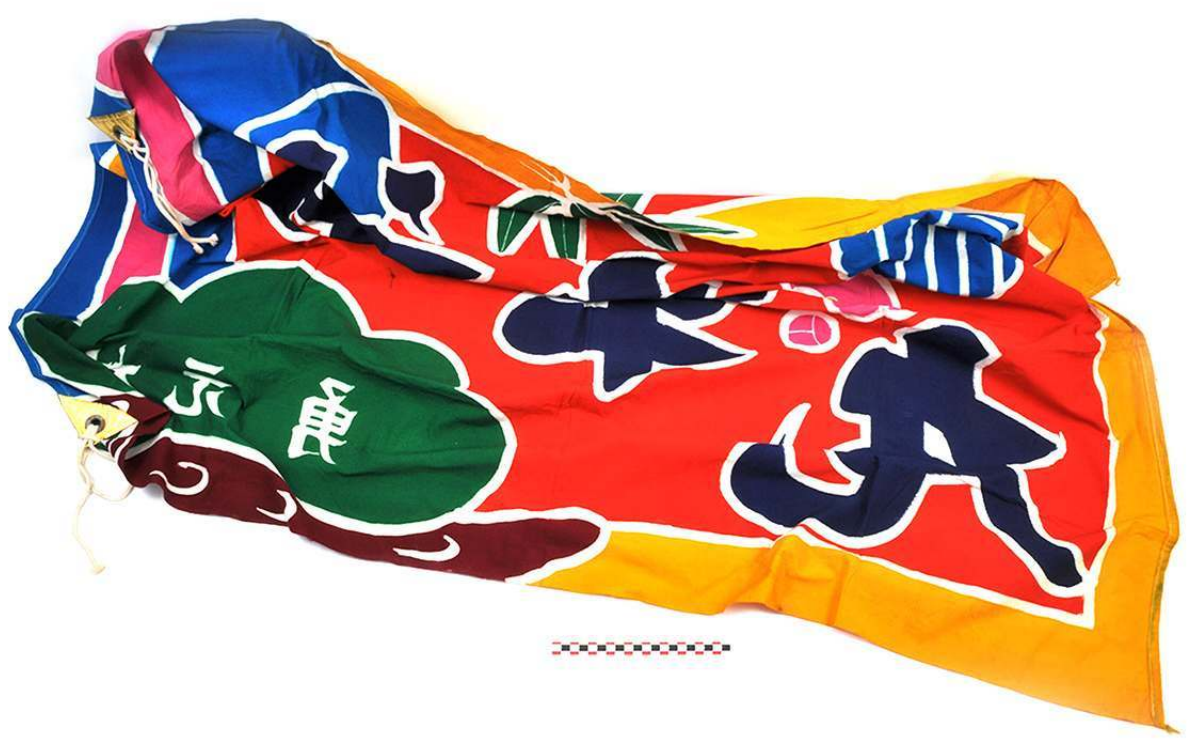

C

(C) P. Rameau

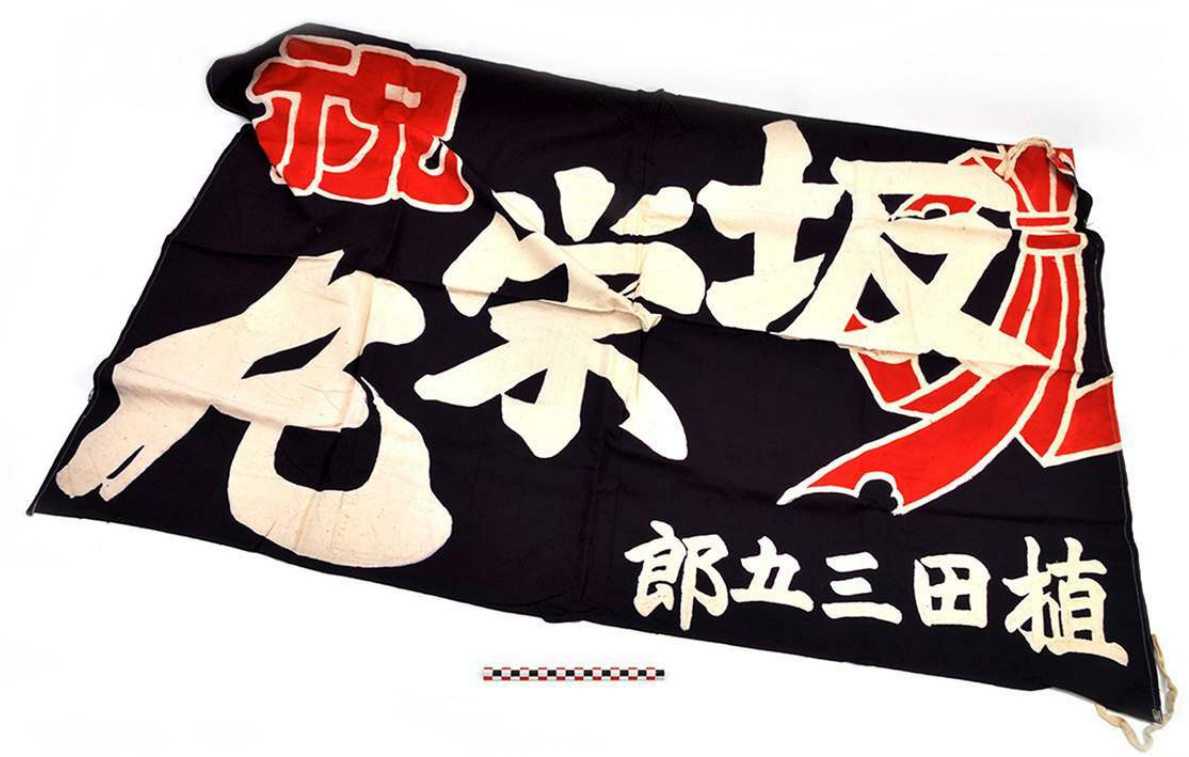

D

(C) P. Rameau

- Japon ; port de Oyanomachi, préfecture de Kumamoto, île de Kyushu.

- Ces étendards nommés Tairyo-bata 大漁旗 ("grande capture-drapeau ») datent des années 1940. Teints à la main, ils portent chance aux pêcheurs. Ils comportent généralement le nom du bateau, avec ou non des dessins traditionnels de bon augure. On les hisse au retour de la pêche pour signifier une prise abondante, ainsi qu'actuellement lors des fêtes de lancement d'un navire.

- Description :

01 : En haut en bas : dessin de grue (tzuru ; Grus japonensis), idéogramme « félicitations » ( iwai), idéogramme " plein de poissons » (tairyo), nom du bateau Yuko maru, et nom du 
commanditaire Kamikawa Zengi. Maintenu verticalement par des ganses, trois sur le petit côté, neuf sur la hauteur, $360 \times 50 \mathrm{~cm}$.

02 : Idéogramme "félicitations"; nom du bateau Sakae maru; nom de personne Senju Matsuba. Attaché par des rubans par le petit côté, $203 \times 124 \mathrm{~cm}$.

03 : Au milieu, nom du bateau Uko Maru ; en bas nom du commanditaire Isamu Kusumoto ; dessins-symboles : bambou (take), prune (ume), épines de pain (matzu). Attaché par des rubans par le petit côté, 156 x $103 \mathrm{~cm}$.

04 : Nom du bateau Sakae maru; nom du commanditaire Sangoro Weda; dessin noshime (symbole de félicité). Attaché par des rubans par le petit côté, 131 x $84 \mathrm{~cm}$.

- 2013 ; collecteur Nicolas Césard

- ETB-JP-NC-2013-01, 02, 03, 04

Figure 20 : Drapeaux TAIRYA-BATA, dans le port de Hachinohe (préfecture d'Aomori) (1/10/2011)

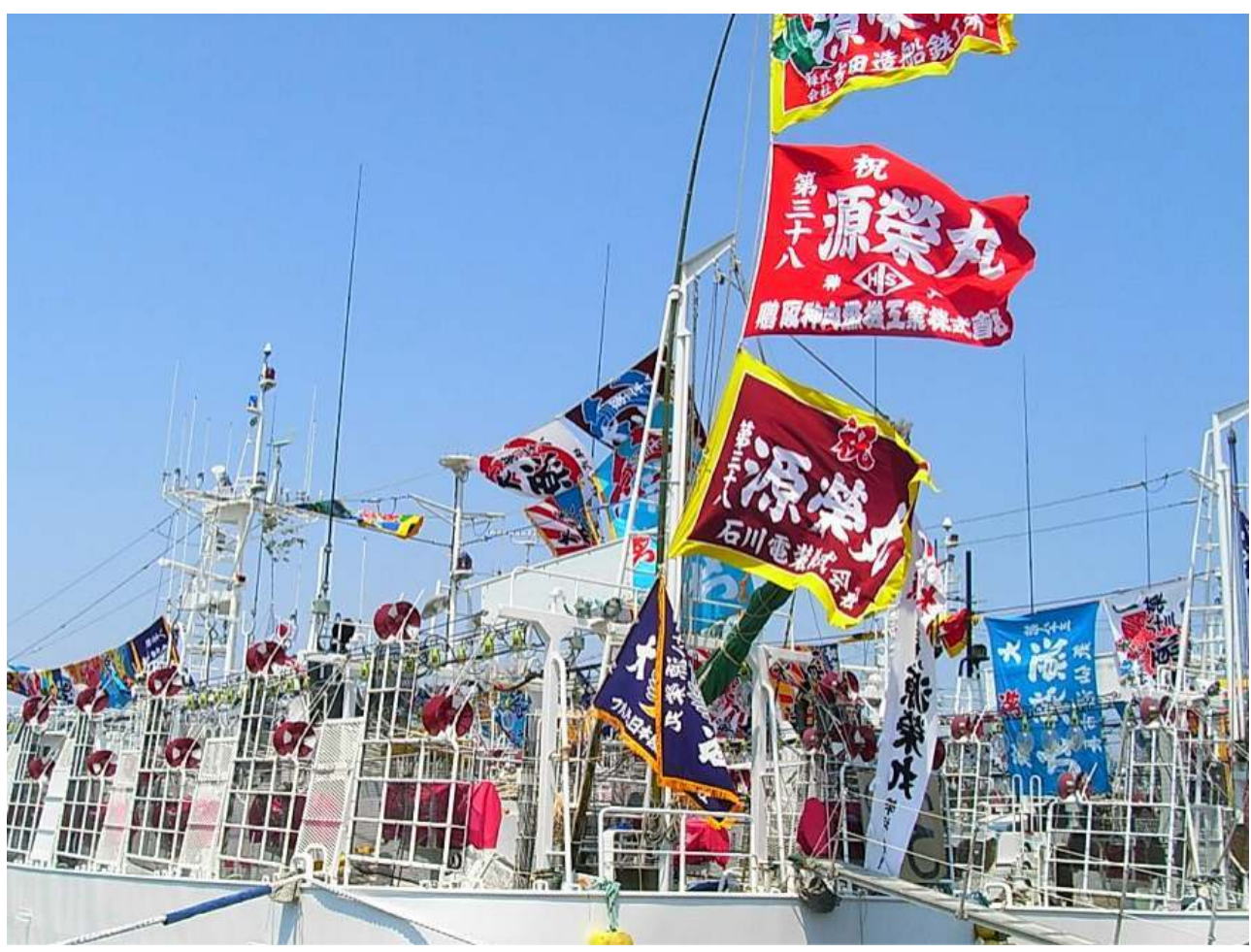

(c) Wikimedia Commons

\section{Techniques de pêche}

\section{Attraction}

Procédé pour attirer

13) Appeau-bruiteur à requins d'Océanie 


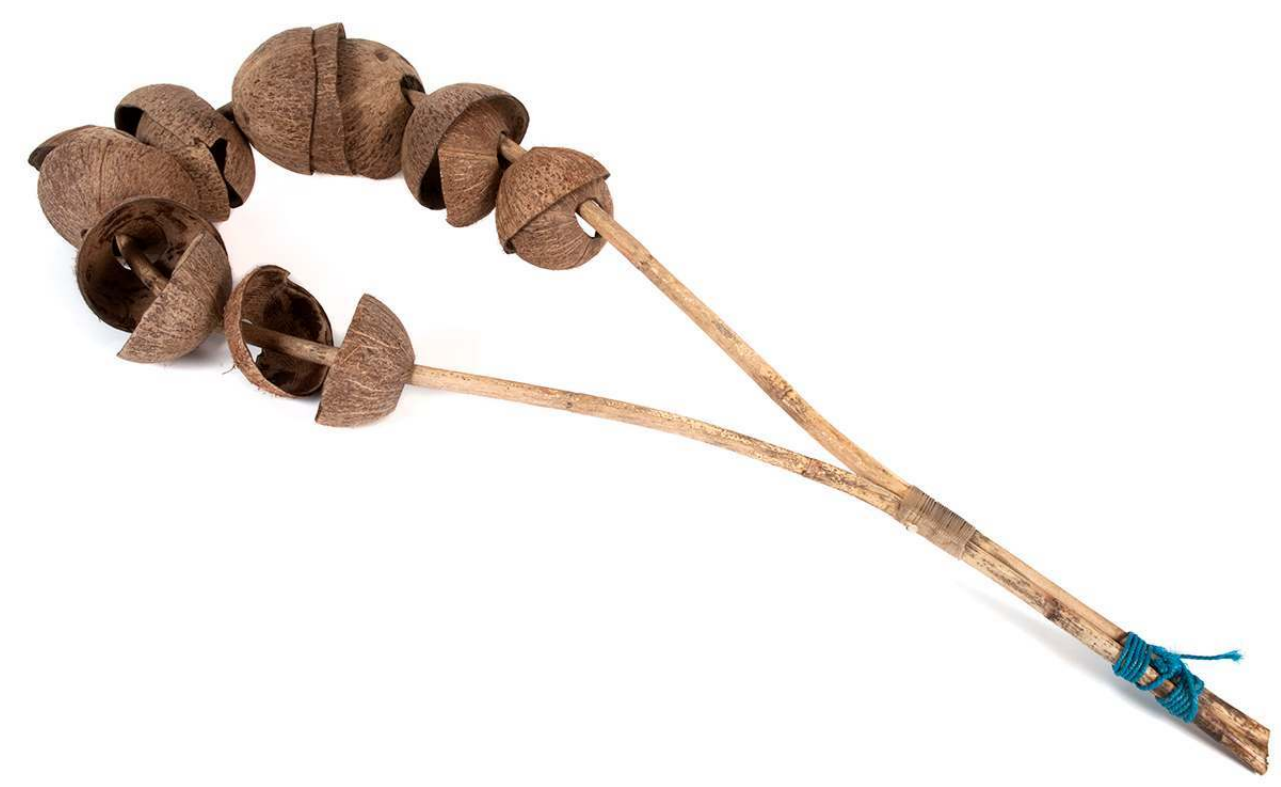

(C) J.-C. Domenech

49 - Îles Salomon; village de Païou, île de Vanikoro ;

- Appeau à requins mango ; fabriqué par Ben Manutaofia, leader de la communauté tikopienne de Païou. L'instrument mango («requin» dans la langue polynésienne de Tikopia) est constitué de demi-coques de noix de coco (fa ngongo) enfilées sur une section de stipe de rotin (loagen) repliée. Cet instrument est utilisé pour la pêche aux requins : le pêcheur l'agite dans l'eau à côté de son embarcation et l'entrechoquement des coques de noix de coco attire les requins qui peuvent alors être ferrés à l'aide d'une ligne munie d'un hameçon appâté avec des morceaux de poissons, ou bien avec un harpon ou même un lasso. Cet instrument est utilisé par les pêcheurs de diverses îles mélanésiennes (Bataille-Benguigui 1981).

-2008, durant la $8^{\mathrm{e}}$ expédition Lapérouse ; collecteur Christian Coiffier

- Longueur $100 \mathrm{~cm}$, largeur $40 \mathrm{~cm}$, épaisseur $15 \mathrm{~cm}$. Manche : longueur $19 \mathrm{~cm}$, largeur $3,5 \mathrm{~cm}$

- MNHN-E-2009.15.19

\section{Capture}

\section{Engins agissant par perforation (Classe IV)}

\section{Perforation balistique}

Engins projetés à la main 


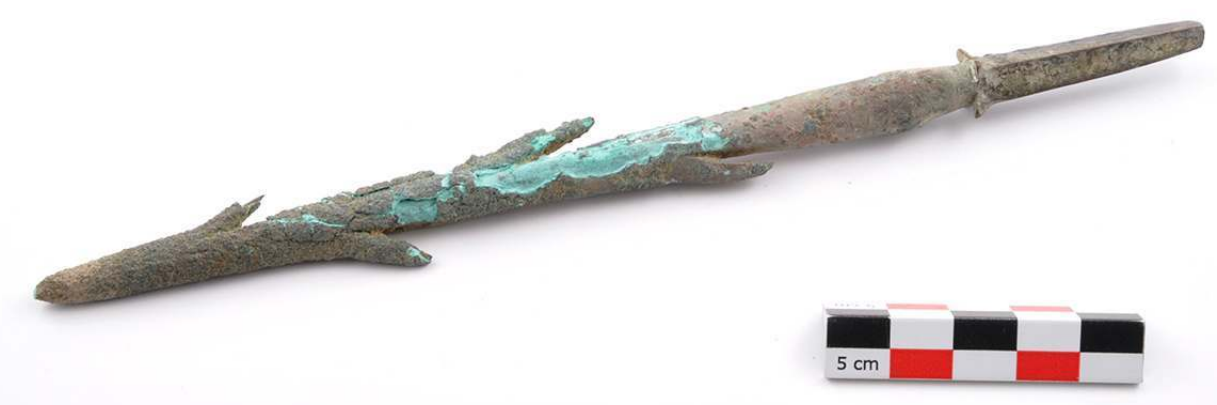

(c) M. Kourdourli

51 - Pérou, Vicús, haut Piura

- Harpon à deux rangs de barbelures en métal avec des traces de cuivre.

- Fouilles 1947-1948, Collecteurs Henry et Paule Reichlen

- Dimensions 20,6 x 1,4 cm

- MNHN-E-2001.40.278.

52 15) Foënes du Brésil

Figure 23 : Foënes du Brésil ETB-BR-MC-2013-005 et ETB-BR-SB-2009-005
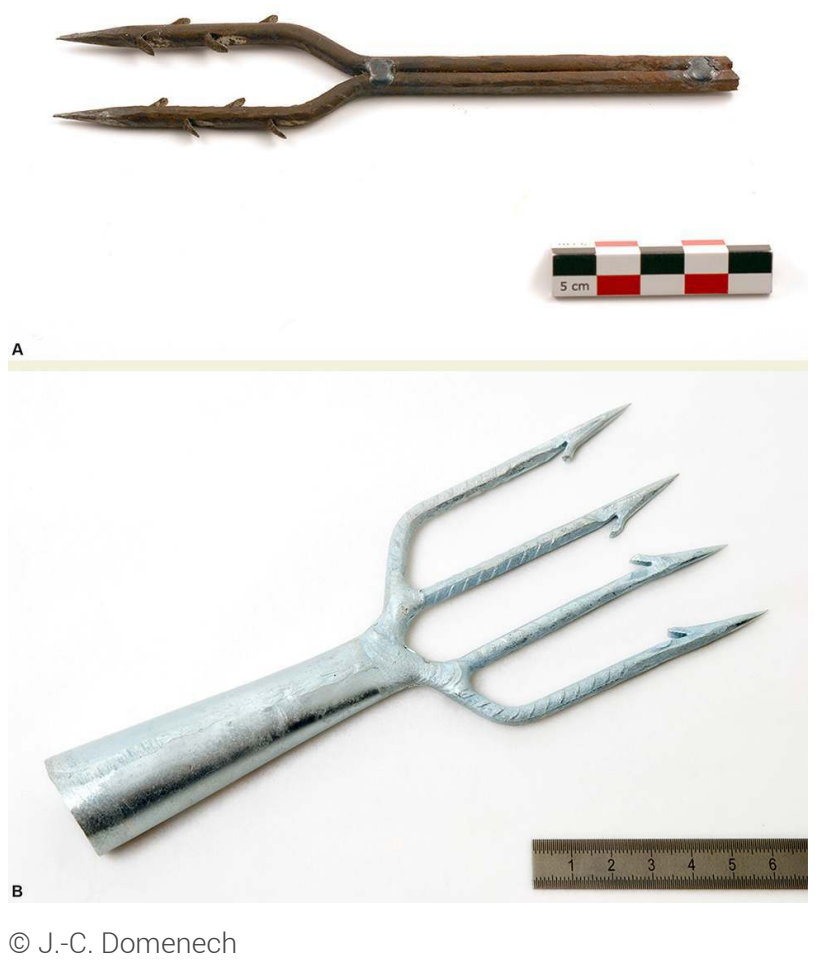

- ETB-BR-MC-2013-005 : Brésil, Parintins, Amazonas

- Harpon en fer à deux pointes pour la pêche en eau douce ; deux tiges de fer soudées 
- 16/04/2013; Achat dans une boutique spécialisée «a casa do pescador»; collectrice Mélanie Congretel

$\cdot 17 \times 3 \mathrm{~cm}$

-ETB-BR-SB-2009-005 : Brésil ; achat à Brasilia

- Foëne en aluminium pour la pêche en rivière

- 2009 ; collecteur Serge Bahuchet

$\cdot 18,1 \times 7,2 \mathrm{~cm}$

54 16) Foënes à anguilles de Vendée

Figure 24 : Foënes à anguilles ETB-FR-RP-2011-069 et 070

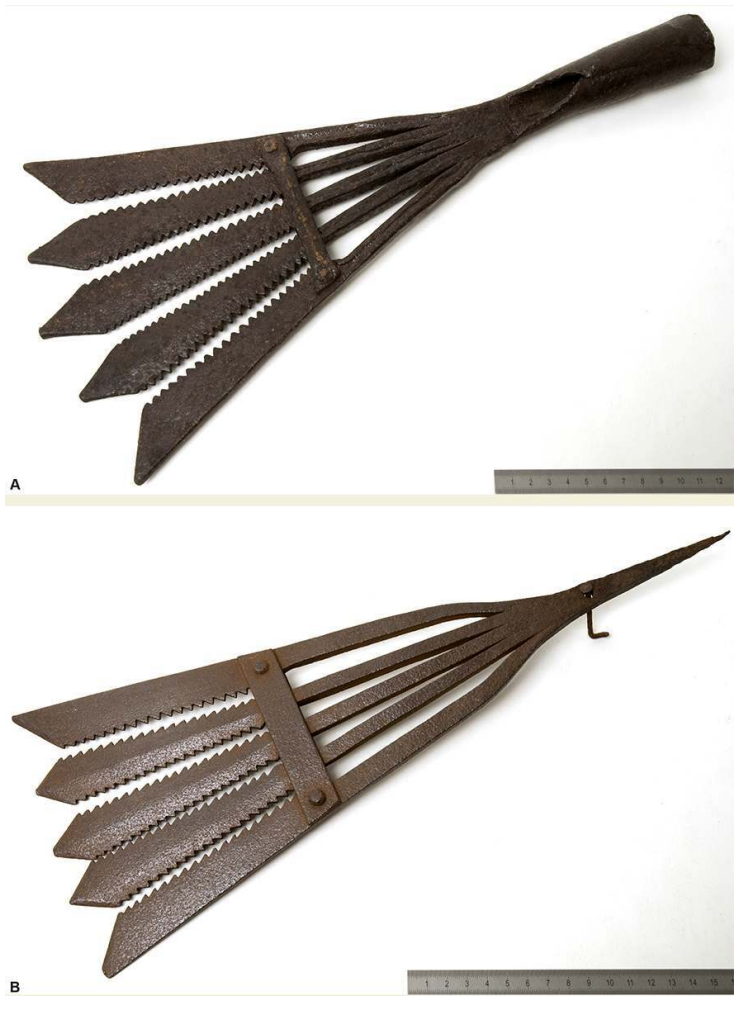

() J.-C. Domenech

55 - France ; la Tranche sur Mer, Vendée

- Foënes plates à anguilles en fer forgé, à 5 lames dentées. ETB-FR-RP-2011-069, emmanchure à douille; ETB-FR-RP-2011-070, emmanchure à soie. Elles étaient utilisées dans le marais poitevin

- Années 1970 ; collecteur Raymond Pujol

- $069: 43 \times 21 \mathrm{~cm} ; 070: 40 \times 16 \mathrm{~cm}$

- ETB-FR-RP-2011-069 et 070 
Figure 25 : La pêche à l'anguille en 1769 selon Duhamel du Monceau (Planche IV Fig. 3)

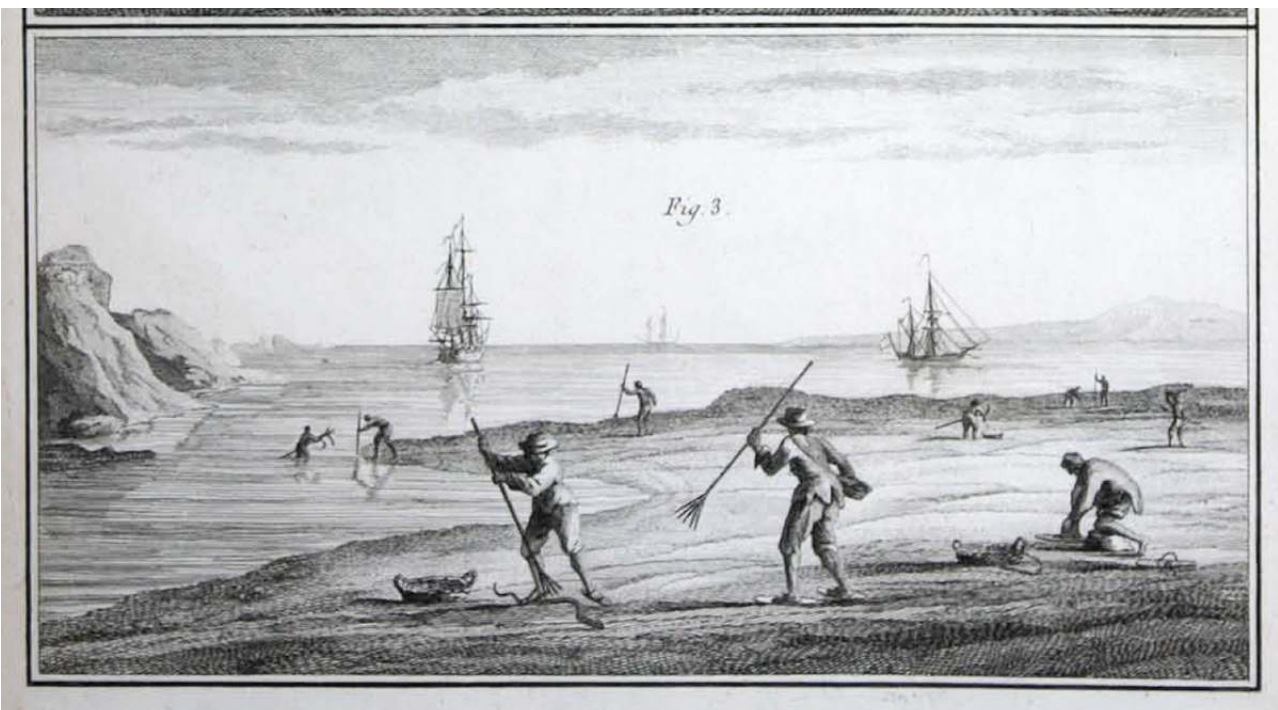

Description de la pêche à l'anguille par Duhamel du Monceau : « En piétinant \& émouvant le terrein sur un fond vaseux, on en fait sortir des Anguilles qu'on prend à la main, qu'on assomme avec un bâton, ou qu'on perce avec une fouanne. Quand les vases sont trop molles pour marcher dessus, les Pêcheurs ajustent sous leurs pieds des planches minces, \& enfonçant au hasard dans la vase des fouannes : ils en retirent des Anguilles \& quelques poissons. » $\left(1769,3^{e}\right.$ section, p. 53).

\section{7) Foëne à anguilles de Vendée}

\section{Figure 26 : Foëne à anguilles ETB-FR-RP-2011-071}

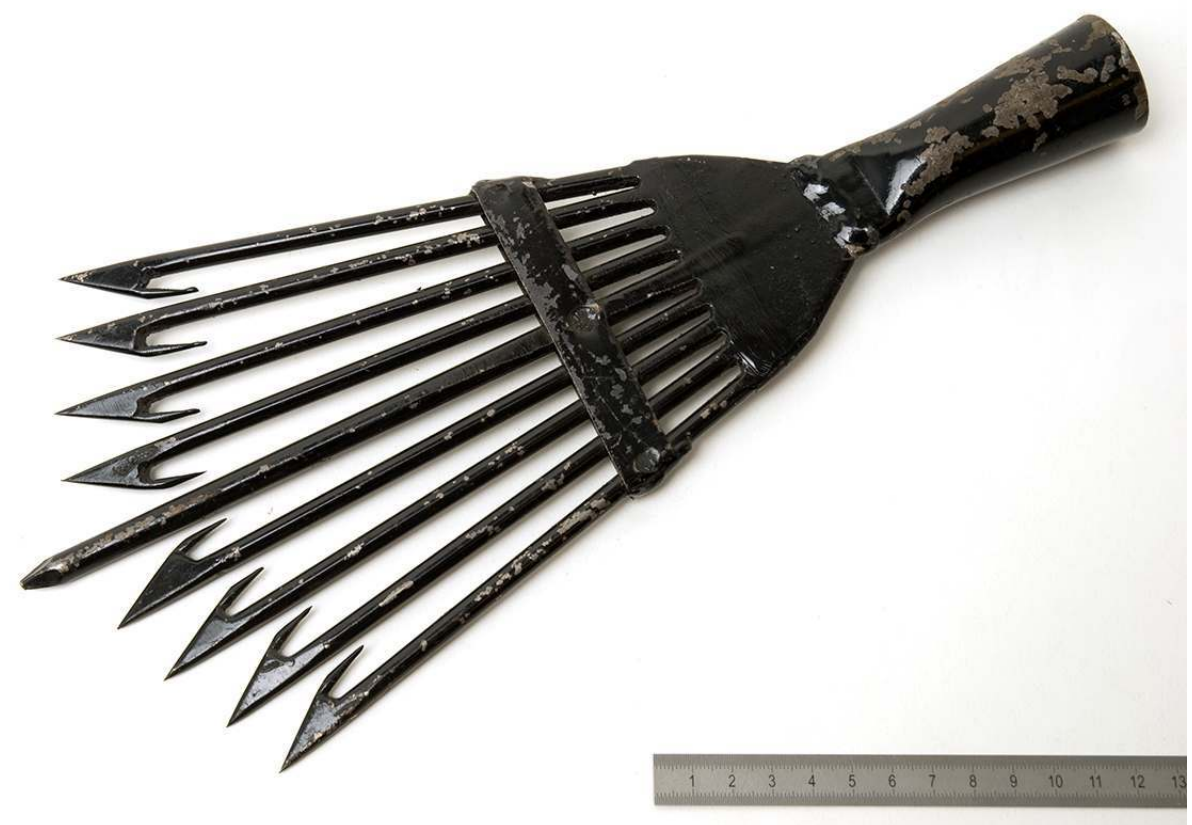

\section{(C) J.-C. DOMENECH}

- France ; la Tranche sur Mer, Vendée

- Foëne à anguilles à 9 pointes en acier forgé et verni. Les fouènes étaient utilisées dans les vasières à marée basse et dans les prairies inondées du marais mouillé 
- Années 1970 ; collecteur Raymond Pujol

- $32 \times 15 \mathrm{~cm}$

• ETB-FR-RP-2011-071

\section{8) Foëne de Croatie}

Figure 27 : Foëne de Croatie ETB-HR-VB-2016-001

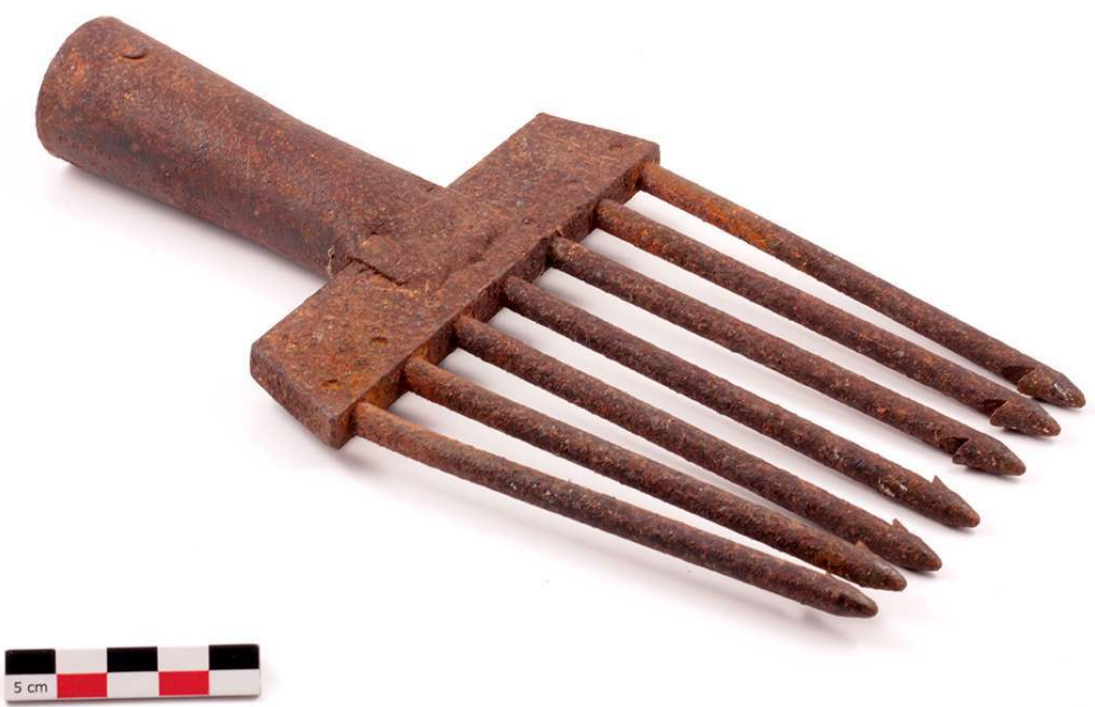

\section{(c) S. Bahuchet}

- Croatie. Île de Lavsa, archipel des Kornati

- Foëne pour lance de pêche osti. Fer usiné. 7 pointes soudées. Se fixe par une douille au bout d'un long manche de bois (ici manquant). La foëne a été trouvée en plongée par $4 \mathrm{~m}$ de fond, près du rivage rocheux de la côte dalmate. Le manche de ce type de lance de pêche mesure de 4 à 8 mètres de long; la pêche se pratique tant du bord que d'un canot, semble-t-il de nuit en attirant les poissons à la lumière. Le nom croate de cette pêche à la lance est lov pod osti ("pêche à la lance ») ou lov pod sviću (" pêche sous la lumière ») (Vuković $2014: 71$ )

- 15 août 2016 ; collecteur Vincent Battesti

- Longueur 24,5 cm, largeur de la foëne $13 \mathrm{~cm}$, diamètre de la douille $3,5 \mathrm{~cm}$

- ETB-HR-VB-2016-001

19) Foëne à main Warao (Venezuela) 
Figure 28 : Foëne à main 2012.7.13(4)

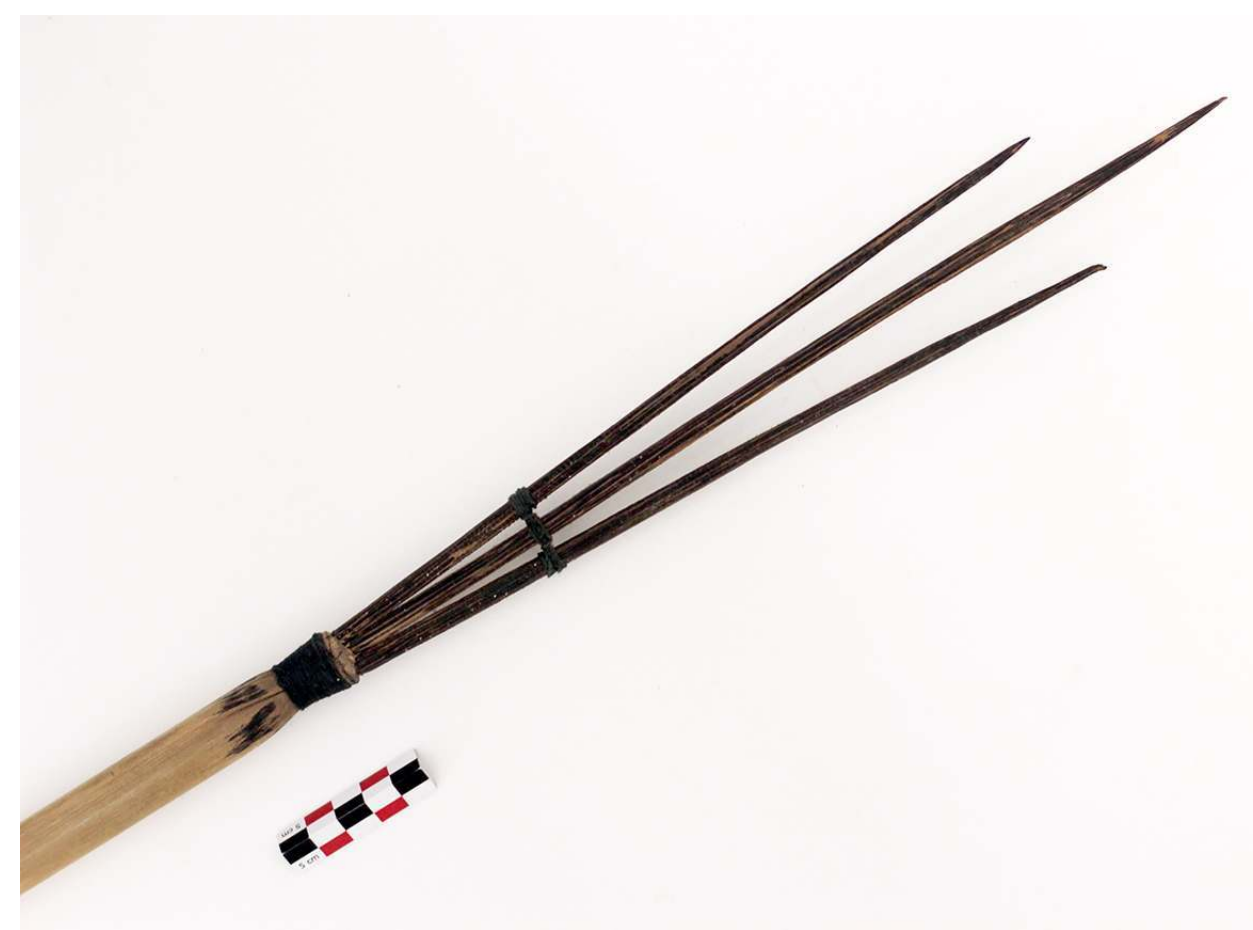

(C) S. Bahuchet

61 - Ethnie Warao. Venezuela, delta de l'Orénoque

- Pointes de bois de palmier, fixées sur une tige de canne à flèche (Gynerium sagittatum, Poacées) et ligaturées avec un fil de coton enduit de résine

- 1974 ; collectrice Brigitte Gladu

- Longueur totale $162 \mathrm{~cm}$, diamètre $2 \mathrm{~cm}$; largeur de la foëne $5 \mathrm{~cm}$

- MNHN-E-2012.7.13(4)

20) Pique à main de Guyane 


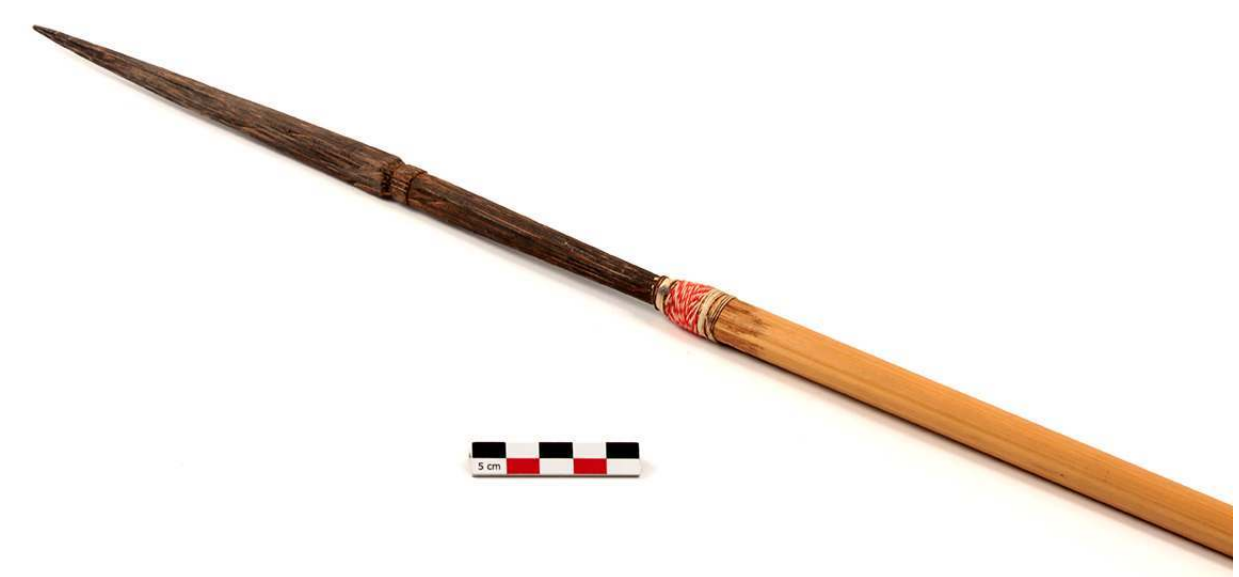

(C) P. Rameau

- Ethnie Wayana. Guyane française, Antecume Pata, Haut Maroni ; acquis au cours de la mission « Nivrée 2000 »

- Pique maniée à la main, notamment lors des pêches collectives à la nivrée, pour recueillir les poissons asphyxiés par le jus de la liane ichtyotoxique Lonchocarpus chrysophyllus (Fabacées) [Sur la pêche à la nivrée, voir plus loin « Paralysie (classe X) », $\mathrm{n}^{\circ}$ 75-76]. Pointe en bois dur de palmier, ligaturée sur une tige de « canne-flèche » (Gynerium sagittatum, Poacées)

- Oct-nov. 2000 ; collectrice Hélène Pagezy

- Longueur $158 \mathrm{~cm}$, pointe $28 \mathrm{~cm}$

- ETB-GF-HP-2000-007

- Références : Pagezy \& Jegu 2004, 2010 
Figure 30 : Usage des piques lors d'une nivrée, à partir de la pirogue, sur le haut Maroni (2000)

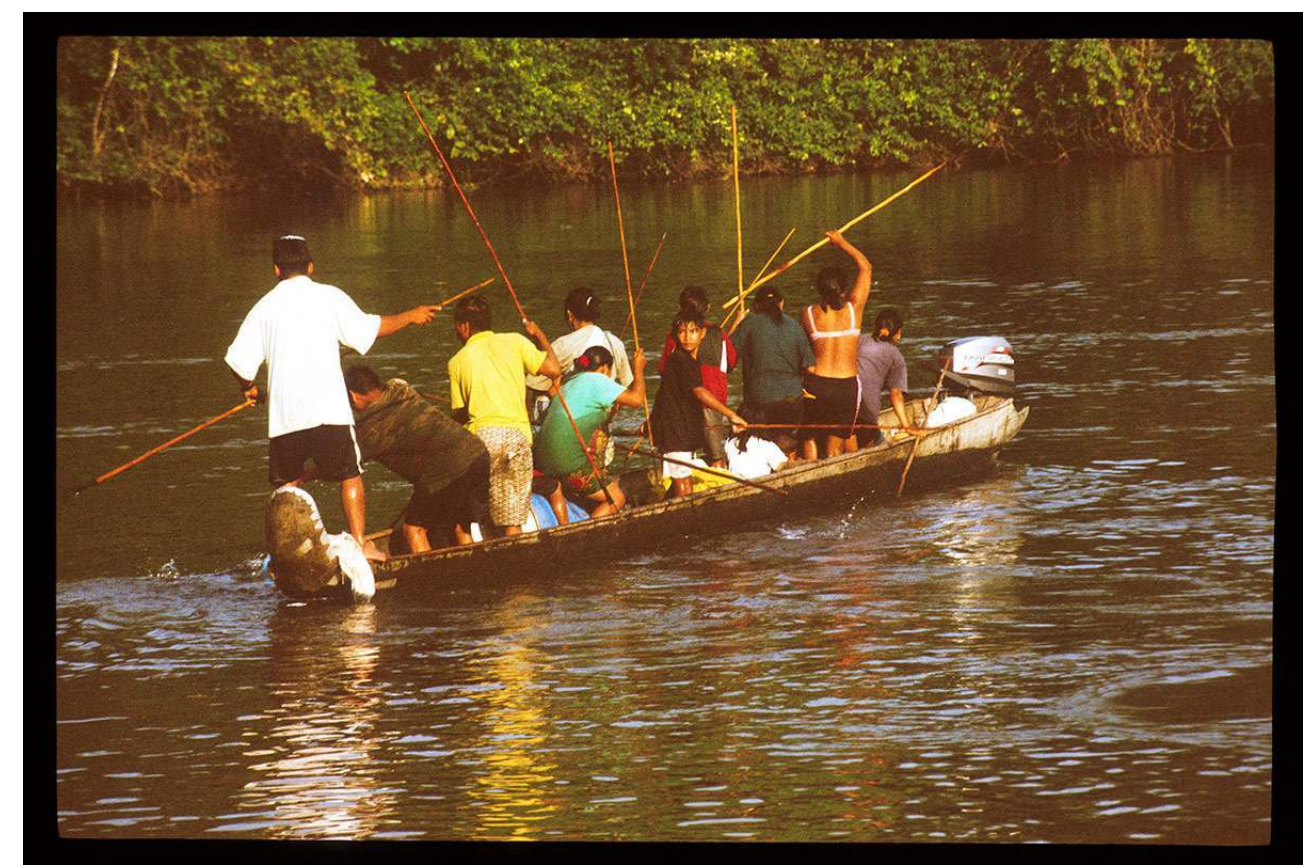

(C) H. Pagezy

\section{Engins projetés à l'arc}

64 21) Flèches de pêche de Guyane

Figure 31 : Trois flèches de pêche wayana ETB-GF-HP-2000-001, 002, 003 et extrémité sans empennage de 001

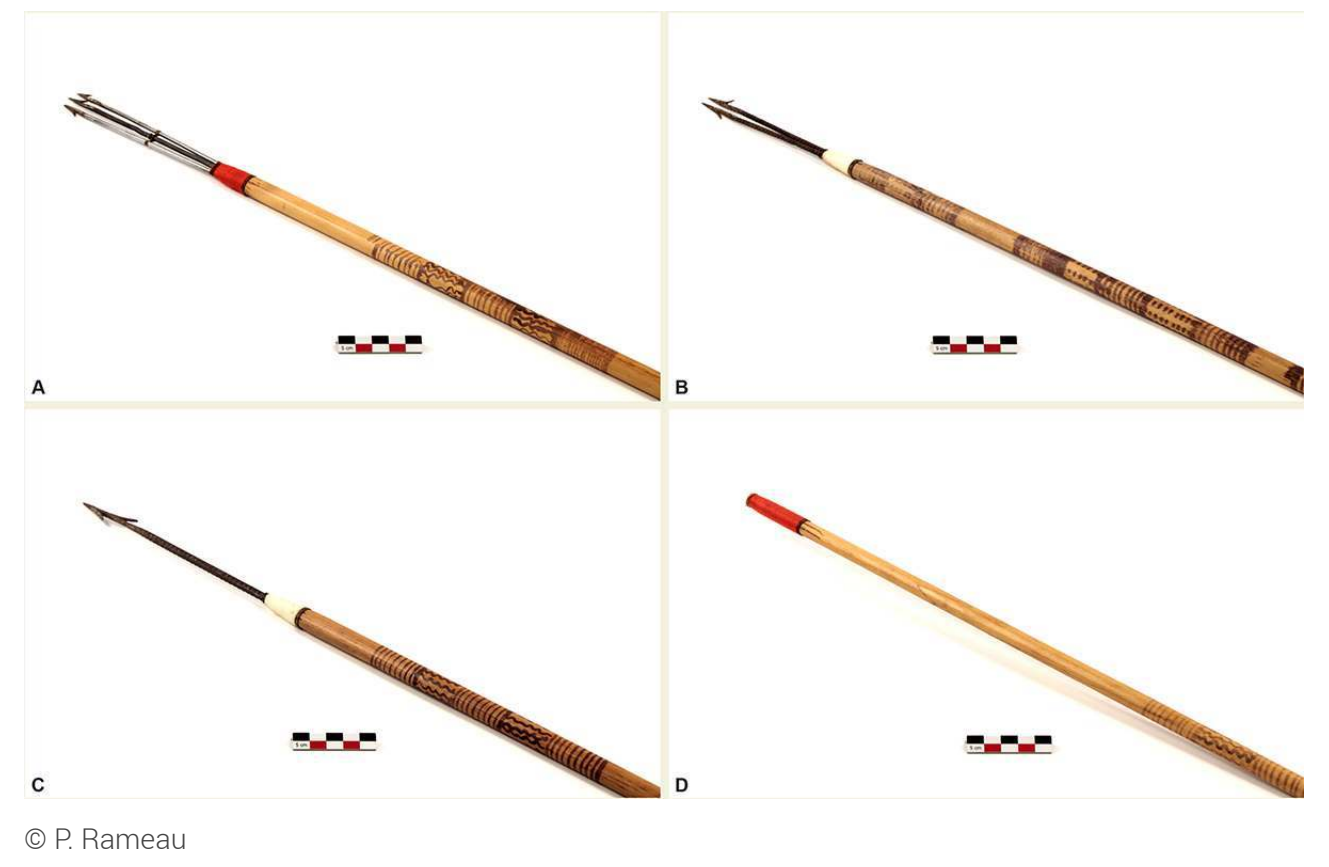

65 - Ethnie Wayana. Guyane française, Antecume Pata, Haut Maroni ; acquis au cours de la mission « Nivrée 2000 » 
- Les pointes sont enfoncées sur une canne à flèche (Gynerium sagittatum, Poacées), dont l'extrémité distale n'est pas empennée, mais seulement ligaturée d'un fil de coton. Elles portent des fers barbelés (de un à trois). Les fûts sont décorés de chevrons teints en sève de Genipa, qui sont aussi des marques personnelles

- Oct-nov. 2000 ; collectrice Hélène Pagezy

- $001: 176 \mathrm{~cm}$, pointe $15 \mathrm{~cm}$; 002 : longueur $169 \mathrm{~cm}$, pointes $12 \mathrm{~cm} ; 003$ : longueur $178 \mathrm{~cm}$, pointe $19 \mathrm{~cm}$

-ETB-GF-HP-2000-001, 002, 003

22) Arc wayana de Guyane

Figure 32 : Détails d'un arc wayana ETB-GF-HP-2000-005

(C) P. Rameau

- Ethnie Wayana. Guyane française, Antecume Pata, Haut Maroni ; acquis au cours de la mission « Nivrée 2000 »

- La partie intérieure de cet arc est plane, l'extérieure est arrondie. Arc en bois de paila ( Brosimum guianense, Moracées), corde en fibres de Bromelia karatas (Bromeliacées) (cf. Grenand 1995 pour l'archerie des Guyanes)

- Oct-nov. 2000 ; collectrice Hélène Pagezy

- Longueur $175 \mathrm{~cm}$

- ETB-GF-HP-2000-005

- Référence : Hurault et al. 1998 
Figure 33 : Wéyoukou pêche avec un de ses fils ; rivière Litani, Saut Poussani (1962)

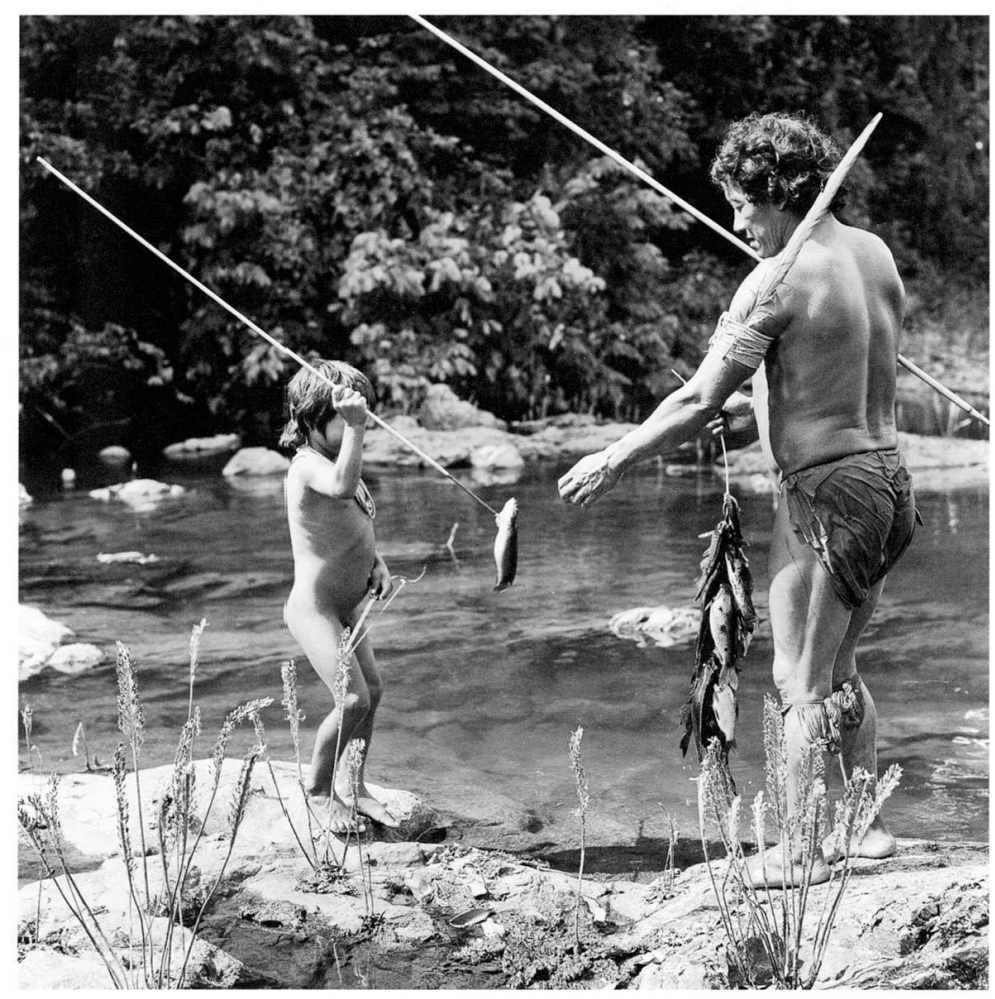

(C) J.-M. Hurault

Figure 34 : Tir à l'arc de pêche pendant la pêche à la nivrée, sur le haut Maroni (2000)

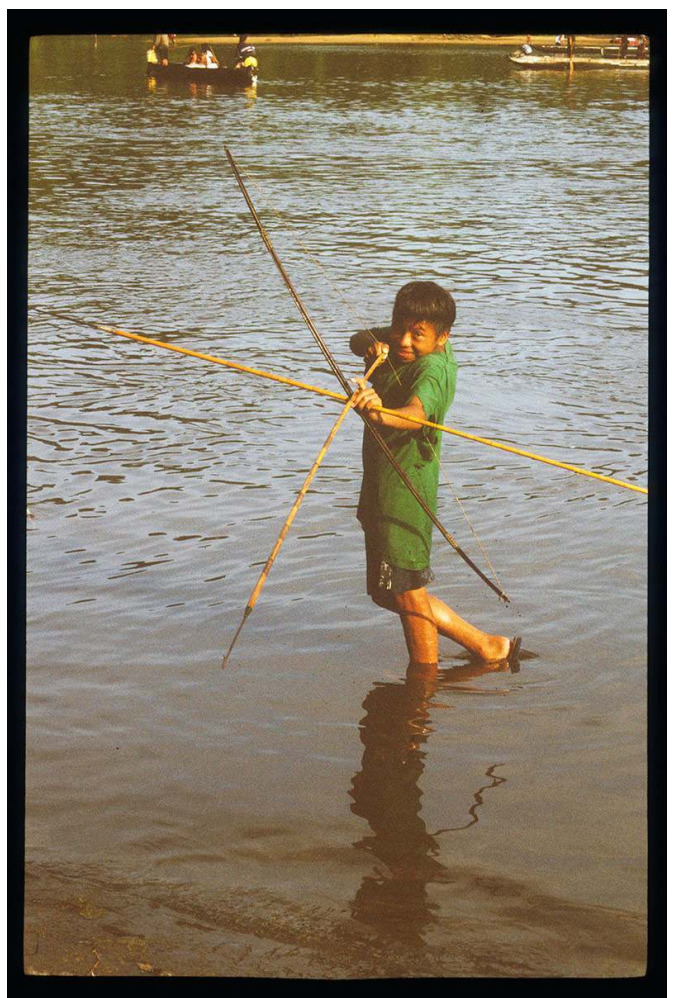

(C) H. Pagezy 
Figure 35 : Pointe de flèche-harpon de pêche kayapo, MNHN-E-2012.3.19

\section{D'après (c) J. Pons-MNHN}

- Ethnie Mebêngôkre. Brésil, village Moikarakô, Terra Indigena Kayapo, État du Para

- Fabriquée par Kaikuare, de la communauté kayapo. Flèche-harpon constituée d'une tige de canne à flèche et comportant une pointe en bois dur avec des entailles crénelées s'amenuisant jusqu'à une pointe, du tiers de sa longueur. Les Kayapo emploient les mêmes flèches, toutes empennées de plumes, pour la pêche aux gros poissons et la chasse au petit gibier terrestre

- 2010-2011; collectrice Pascale de Robert

- Longueur $63 \mathrm{~cm}$, diamètre env. 0,2 cm

- MNHN-E-2012.3.19 (exposé dans la Galerie des enfants à la Grande Galerie de l'Évolution MNHN)

Figure 36 : Pêcheur à l'arc kayapo

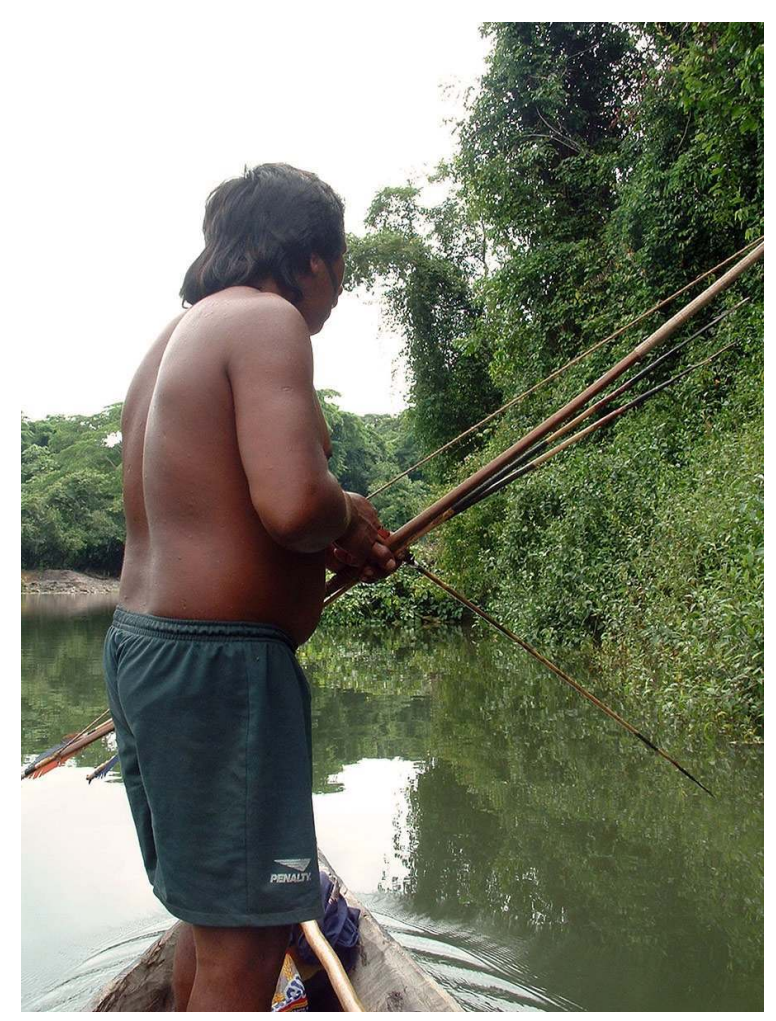

(c) MNHN ; cliché F.M. Le Tourneau

24) Flèches warao (Venezuela) 


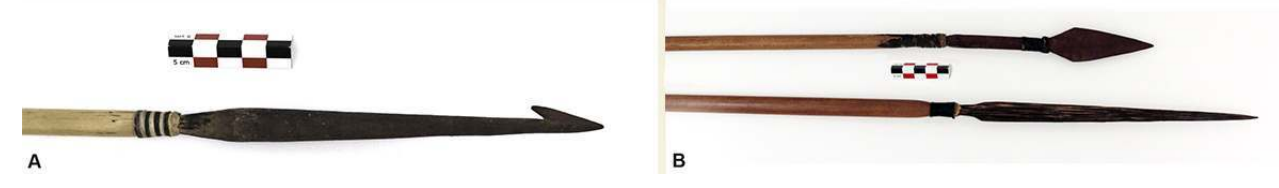

71 - Ethnie Warao. Venezuela, delta de l'Orénoque

- Toutes les pointes sont montées sur de la canne à flèche. MNHN-E-2012.7.13(3) : bois de palmier, avec une barbelure ; MNHN-E-2012.7.11(2) pointe en bois sur un support en bois dur ; (3) bois de palmier

- 1974 ; collectrice Brigitte Gladu

- 2012.7.13(3) : longueur totale $109 \mathrm{~cm}$, pointe $16 \times 1,8 \mathrm{~cm} ; 2012.7 .11(2)$ : longueur totale $134 \mathrm{~cm}$, pointe $17 \times 3 \mathrm{~cm} ; 2012.7 .11(3)$ : longueur totale $141 \mathrm{~cm}$, pointe $24,2 \times 2 \mathrm{~cm}$

- MNHN-E-2012.7.13(3), MNHN-E-2012.7.11(2,3)

\section{Perforation non balistique}

72 L'efficacité de la pêche à " perforation non balistique », donc sans projection vers la proie, repose essentiellement sur deux paramètres : d'une part l'attraction de la proie, c'est-àdire l'appât (boëtte ou esche en langage technique), et d'autre part sur le mouvement de l'hameçon, adapté au comportement de l'espèce ciblée.

73 Dans cette sous-classe d'engins, Théodore Monod distingue les hameçons dont l'action est périphérique et somatique, c'est-à-dire que c'est l'ensemble du corps qui est agrippé (les «turluttes»), de ceux dont l'action est localisée et buccale (le poisson «mordant à l'hameçon » selon l'expression consacrée !).

\section{Action périphérique somatique}

74 Les turluttes sont des engins particulièrement destinés à la capture des seiches et calamars, d'où leur autre nom de «calamarette ». Avec ou sans appât, leur forme ovale et surtout leur mouvement, vertical, attirent les proies, qui les enserrent de leurs tentacules. Elles sont généralement attachées en chaîne. Elles comportent une couronne de pointes nommée "panier ». Le plus souvent on les emploie de nuit, avec une vive lumière qui attire les céphalopodes.

25) Turlutte 


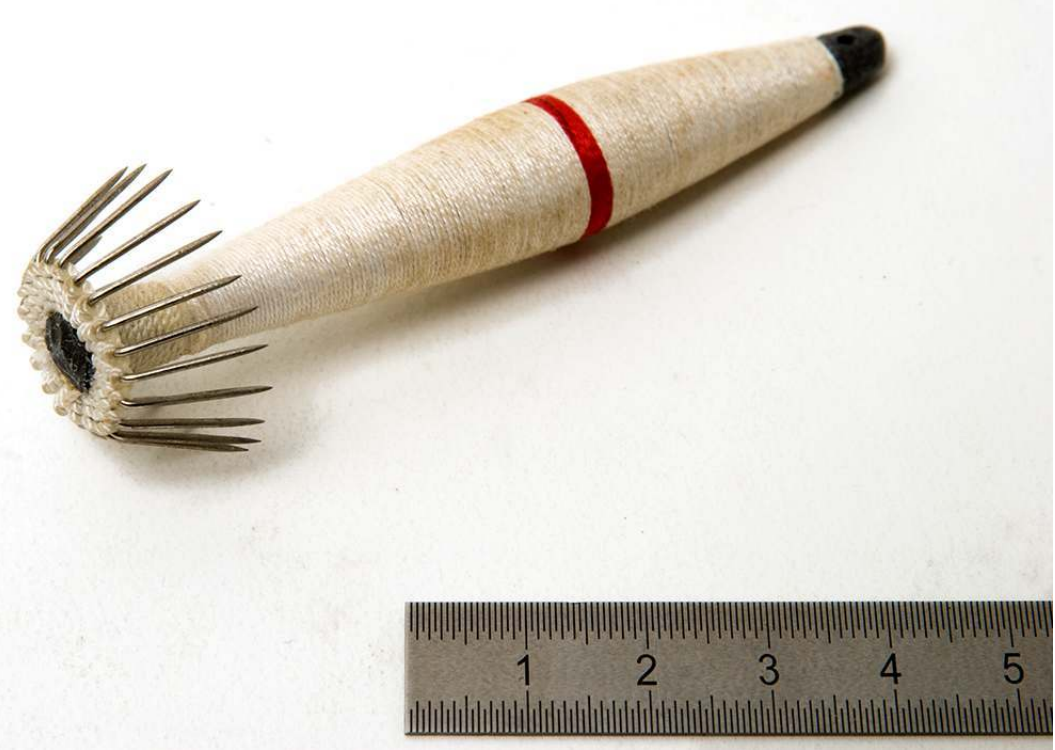

(C) J.C. Domenech

76 - Espagne ; Ibiza

- Turlutte - Corps en plomb, entouré de fil de coton

- Années 2000 ; collectrice Florence Brunois

- 8,5 x 2,5 cm

- ETB-ES-FB-2018-001

77 26) Turlutte-cage 


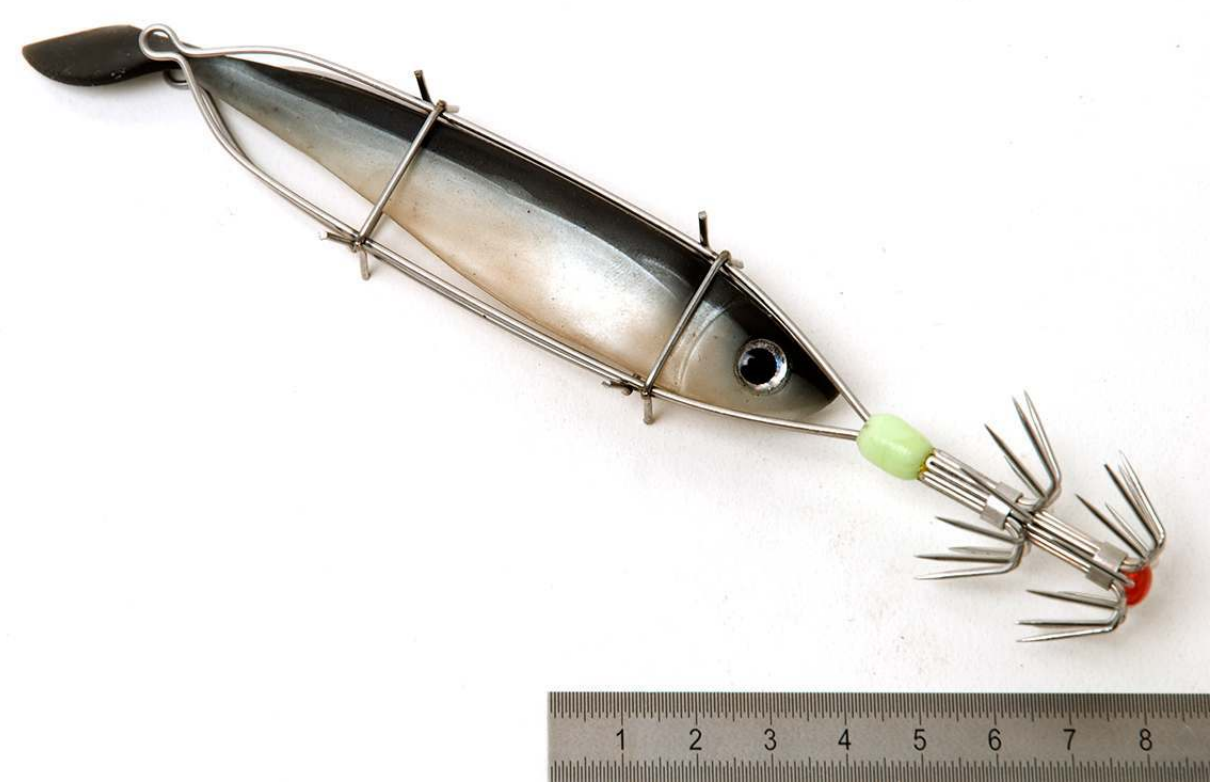

\section{(C) J.C. Domenech}

- France ; Douarnenez, Finistère ; achat à la coopérative maritime

- Turlutte-cage à deux paniers et appât artificiel. 2 éléments différents : la turlutte et l'appât. («Pour boëtter avec un poisson (sardine, sprat, tacaud...»). "Turlutte-cage, totonara ", marque Flashmen. Pour les seiches.

-2006, collecteur Serge Bahuchet

- Cage 16 x 2,5 cm, diamètre panier $3 \mathrm{~cm}$

• ETB-FR-SB-2018-001 (turlutte) ; ETB-FR-SB-2018-002 (appât)

27) Turluttes à seiches

Figure 40 : Trousse de 10 turluttes ETB-FR-SB-2018-003 et détail d'un hameçon
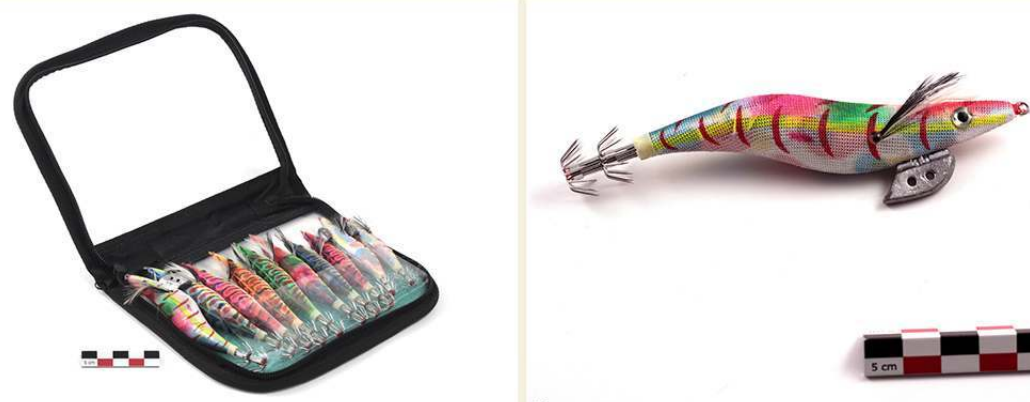

(C) A- P. Rameau ; B- S. Bahuchet

- France, Arcachon, Gironde ; achat à la coopérative maritime

- Trousse de 10 hameçons à seiches avec leurre ; marque Squid Master

- Février 2012 ; collecteur S. Bahuchet (don de la promotion EDTS 2012)

- Trousse : 20,5 x $16 \mathrm{~cm}$, longueur de la plus grande turlutte $13 \mathrm{~cm}$, de la plus petite $8 \mathrm{~cm}$

- ETB-FR-SB-2018-003 


\section{Action localisée (buccale)}

Il s'agit dans cette famille de l'ensemble des techniques utilisant un hameçon, une pièce pointue, souvent courbe, que la proie va ingurgiter et à laquelle elle se trouvera suspendue par la bouche à un fil.

Le mode de maintien de la ligne par le pêcheur, et le type d'accrochage, permettent de distinguer des catégories, telles que les classiques "pêche à la ligne » ou "palangre ». Nous ne les négligerons pas. Toutefois, une grande division nous semble pertinente, car elle implique la relation du pêcheur à sa proie: soit le pêcheur est présent lors de la pêche, la distance avec sa proie est minimale et il anime lui-même la ligne (et l'on parlera alors de "pêche directe») ; soit au contraire la pêche s'effectue en l'absence du pêcheur qui aura placé son engin afin qu'il agisse seul; la distance au poisson est maximale, on parlera alors de "pêche indirecte » (Tableau 2).

3 En revanche, dans les deux cas, le même soin est apporté à l'emplacement de l'hameçon par rapport à l'habitat de la proie, et au type d'appât qui devra l'attirer. En tout premier lieu, la profondeur où doit se placer l'hameçon : à la surface, entre deux eaux ou au fond. Cela se règle subtilement grâce à un flotteur dans lequel la ligne est coincée à la longueur adéquate, des poids plus ou moins lourds et nombreux maintenant l'hameçon enfoncé.

Tableau 2 : Types de pêche avec des hameçons

\begin{tabular}{|c|c|c|}
\hline \multirow{4}{*}{ Pêche directe } & A la main [sans canne] & \\
\hline & \multirow[b]{2}{*}{ Avec canne } & Hameçon plongé, avec plombs et flotteurs \\
\hline & & $\begin{array}{l}\text { Hameçon affleurant, animé à la surface [sans plomb, technique de } \\
\text { " la mouche "] }\end{array}$ \\
\hline & Avec des flotteurs [lignes flottantes, dérivantes, que l'on surveille] & \\
\hline \multirow{3}{*}{ Pêche indirecte } & Flottantes & \\
\hline & Plongées & \\
\hline & «Lignes automatiques" & \\
\hline
\end{tabular}

On distinguera donc:

Pêche directe : techniques où le pêcheur est présent [du bord, d'une embarcation]

À la main, sans canne

Avec canne

Hameçon plongé, avec plombs et flotteurs

Hameçon affleurant, animé à la surface (sans plomb, technique de « la mouche »)

Avec des flotteurs, lignes flottantes, dérivantes, que l'on surveille

Pêche indirecte : techniques où le pêcheur est absent

« lignes sédentaires; dormantes ", disposées et relevées après un espace de temps

Flottantes, lignes dérivantes, palangres de surface

Plongées, corps pesants avec différents supports (palangres calées)

«Lignes automatiques" (pièges à déclencheur: la ligne est maintenue plongée par un dispositif qui s'ébranle quand le poisson tire sur l'hameçon; il se trouve ainsi projeté en l'air ; technique similaire à celle des pièges à collet terrestres).

Toutes ces catégories ne sont pas représentées dans nos collections.

- Les hameçons archéologiques

\section{8) Hameçons archéologiques d'Amérique latine}

Les collections ethnologiques conservent quelques pièces archéologiques résultant des recherches de Henry et Paule Reichlen au Pérou et de Guy Stresser-Péan au Mexique. 
C'est la forme des objets qui par analogie permet de leur attribuer une fonction halieutique. Mais dans certains cas le doute est permis; telle pièce courbe est-elle un hameçon ou un crochet servant à un tout autre usage ? Telle pointe barbelée est-elle un réel harpon de pêche ou une flèche de chasse ( $c f . n^{\circ} 14$ dans ce catalogue) ? Par ailleurs nous n'avons pas trouvé d'informations chronologiques sur ces objets.
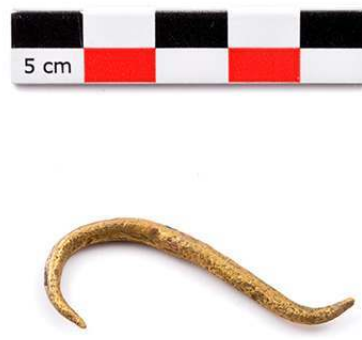

(C) M. Kourdourli
91

- MNHN-E-2001.40.403 : Pérou ; site de Balsas, département d'Amazonas, haute vallée de l'Utcubamba

- Hameçon en cuivre doré. Civilisation Chimu

- Fouilles 1948, Collecteurs Henry et Paule Reichlen

- Longueur 4,1 cm, largeur $0,3 \mathrm{~cm}$

- MNHN-E-2001.40.299 : Pérou ; Vicús, haut Piura

- Hameçon en métal

- Fouilles 1947-1948, Collecteurs Henry et Paule Reichlen

- Dimensions 5 x 0,7 cm 


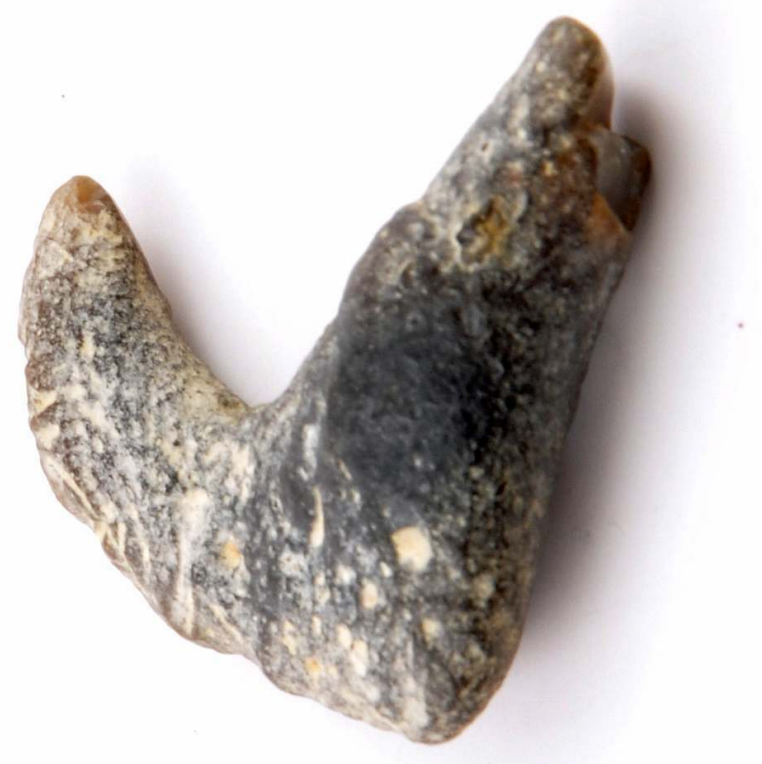

(c) M. Kourdourli

- Mexique ; Tampaxal, État de San Luis Potosi

- Hameçon en pierre

- Fouilles de G. Stresser-Péan

- Dimensions 2,8 x 2,5 cm, épaisseur 0,8 cm

- MNHN-E-2002.2.153

\section{- Les hameçons modernes}

De nos jours, les magasins de pêche de tous les pays du monde vendent des hameçons qui ne sont fabriqués que par une douzaine de compagnies, de quelques pays producteurs seulement (Japon, USA, Chine, Norvège, Grande Bretagne, Finlande et même France). On assiste de ce fait à une diffusion universelle des hameçons industriels. Chaque producteur propose un large catalogue où les hameçons diffèrent par le type de métal et de revêtement, l'angle, le nombre, la longueur et la courbure des pointes, la forme de l'ergot (« l'ardillon ») et le mode d'accroche (anneau ou palette). 
Figure 43 : Terminologie des hameçons dans le catalogue de la marque VMC ${ }^{\circ}$

CHOISIR LE BON HAMECON VMC

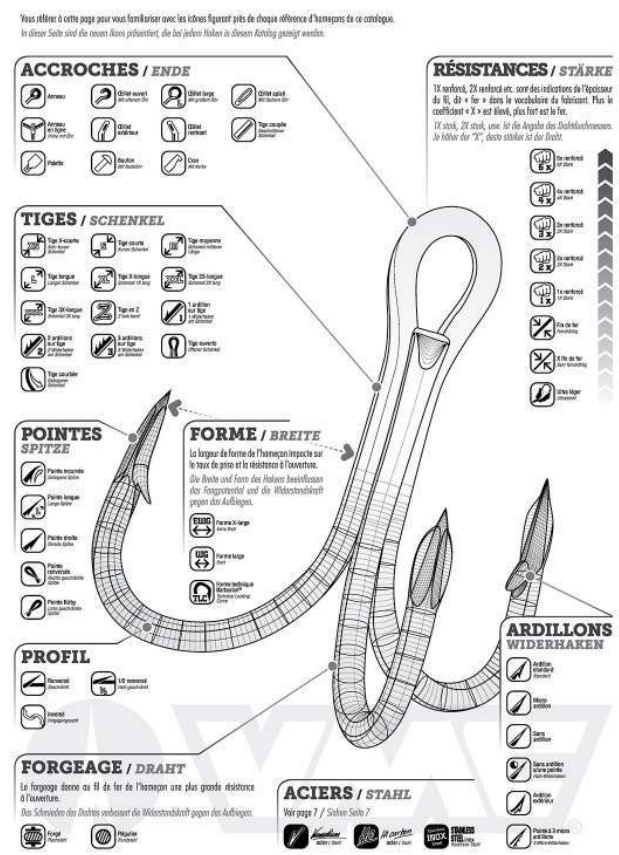

(c) VMC

29) Lots de hameçons de tailles et de formes diverses (Mexique, Pérou, France)

97 Taille et forme des hameçons sont choisies par les pêcheurs en fonction des types de poissons qu'ils convoitent. Selon la taille, les hameçons sont vendus à la pièce ou en lots. 


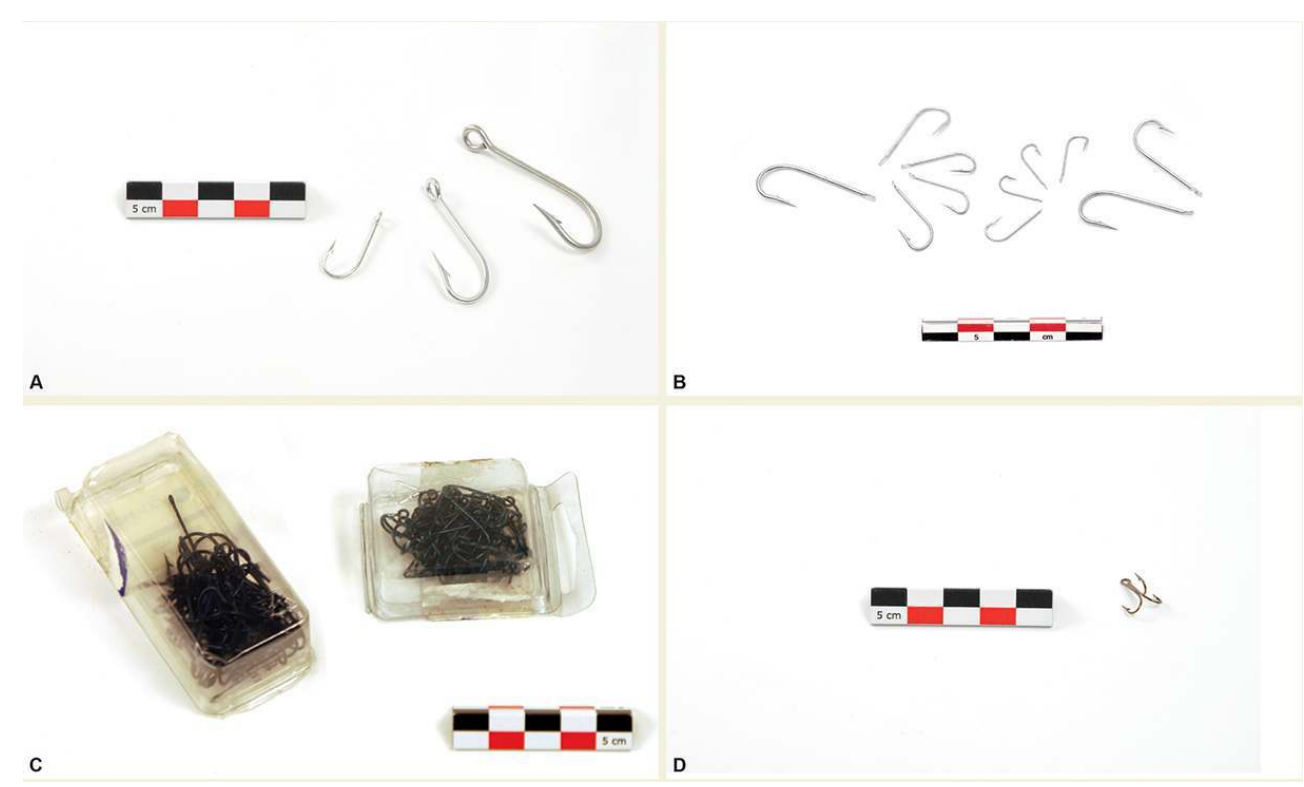

A- ETB-MX-FA-2009-12 (Mexique ; Manzanillo, Colima ; 11/2009; collectrice Françoise Aubaile ; longueur 2,5, 4 et $5 \mathrm{~cm}$ ) ; B- ETB-PE-MQ-2009-030 (ANZUELOS ; Pérou ; Salaverry, La Libertad ; 20/08/2009 ; collectrice Marina Quiñe) ; c- ETB-PE-MQ-2009-044 (Pérou ; Salaverry, La Libertad ; 20/08/2009 ; collectrice Marina Quiñe ; marque Aquahook, deux boîtes de 100) ; D- Hameçon triple ETB-FR-RP-2012-056 (France, collecteur R. Pujol ; longueur 1,5 cm).

(c) P. Rameau et J.C. Domenech

\section{0) Hameçon en nacre d'Océanie}

Figure 45 : Hameçon d'Océanie ETB-VU-FB-2006-001

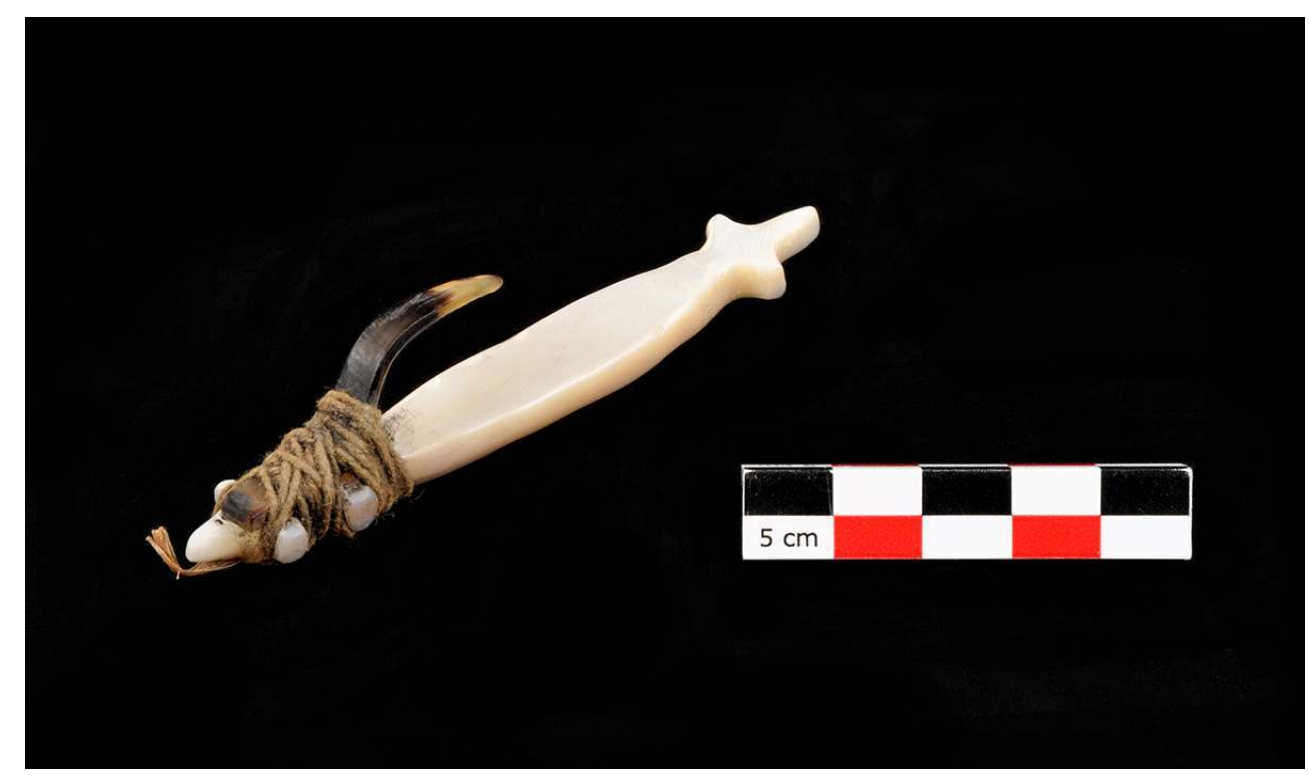

(C) P. Rameau

99 - Vanuatu, île de Santo

- Hameçon en nacre. Nacre et crochet en écaille de tortue ; en même temps le mouvement de la ligne fait tournoyer l'hameçon, leurrant la proie

- Octobre 2006 ; collectrice Florence Brunois 
- Longueur $9 \mathrm{~cm}$, largeur $1,5 \mathrm{~cm}$, épaisseur du crochet $2 \mathrm{~cm}$

- ETB-VU-FB-2006-001

31) Hameçon des îles Salomon

Figure 46 : Hameçon des îles Salomon

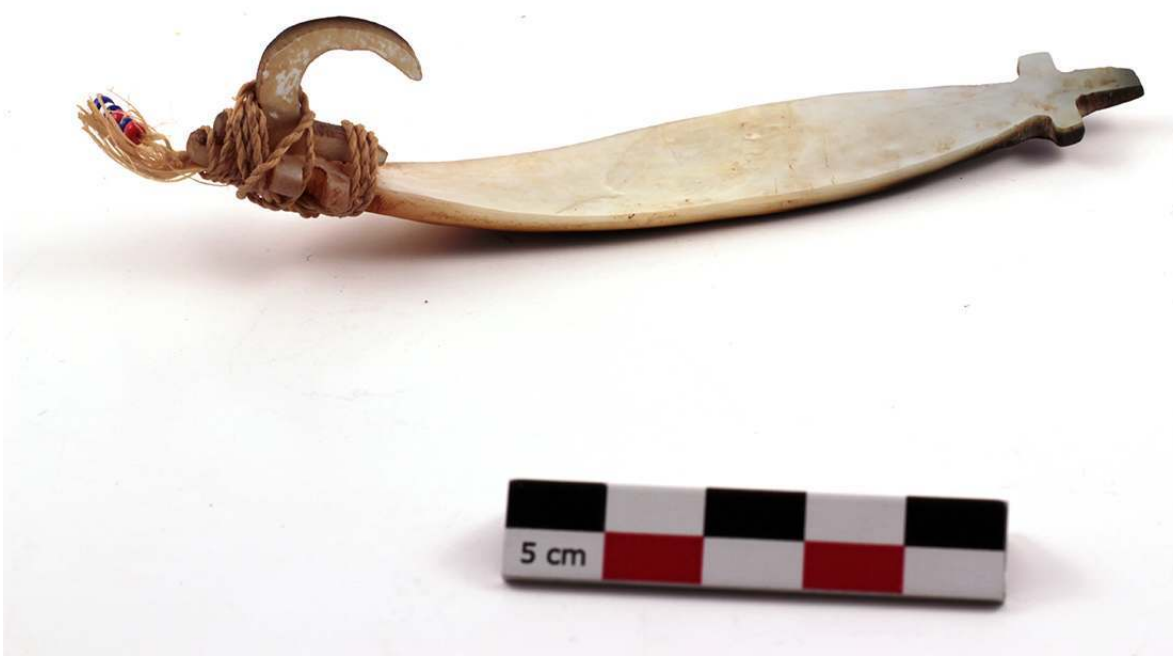

(c) S. Bahuchet

101 • Îles Salomon. Achat à Nouméa

- Hameçon en nacre. Ligature en corde

- 2015 ; collecteur Antoine Lonnet, don Françoise Aubaile

- Longueur $12 \mathrm{~cm}$, largeur $205 \mathrm{~cm}$, hauteur $2,4 \mathrm{~cm}$

- ETB-NC-FA-2018-001

32) Leurre en forme d'un poulpe 


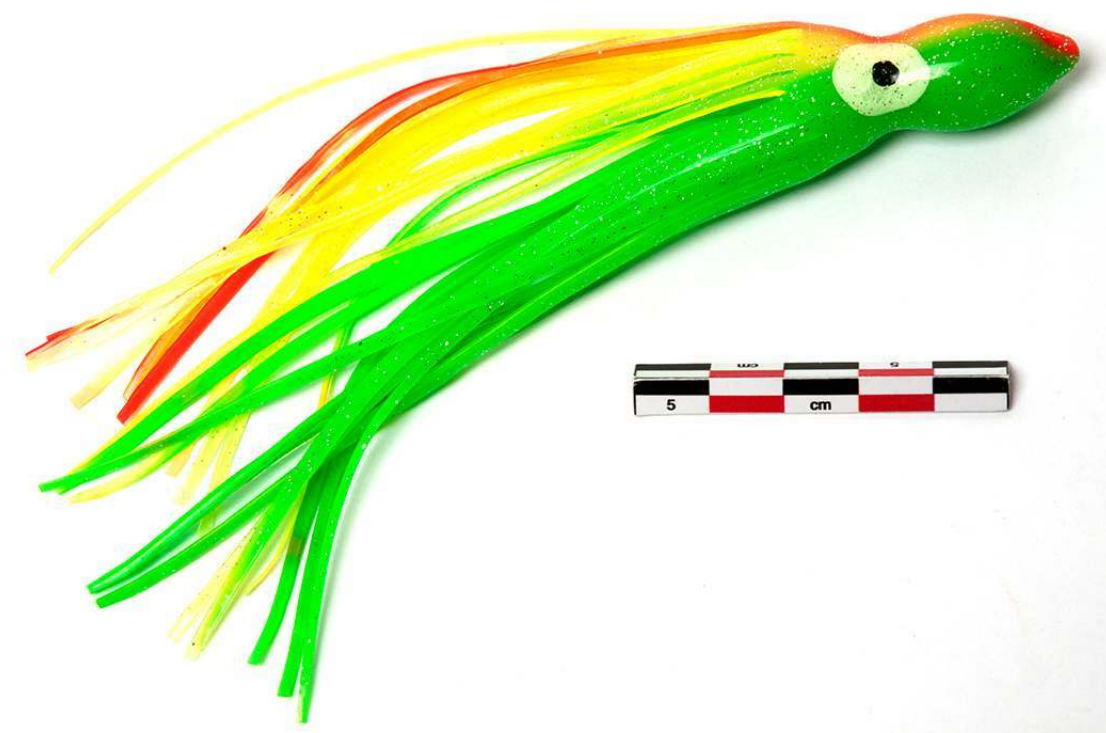

(C) P. Rameau

- Mexique. Guadalajara, État du Jalisco.

- Señuelo. Leurre en plastique coloré en forme de poulpe, pour les grands poissons de mer. Se fixe sur un hameçon

-11/2009 ; collectrice Françoise Aubaile

- Longueur $15 \mathrm{~cm}$

- ETB-MX-FA-2009-010

33) Représentation de la pêche du dieu Aia-Paec 
Figure 48 : Représentation de la pêche sur une calebasse ETB-PE-MQ-2009-009

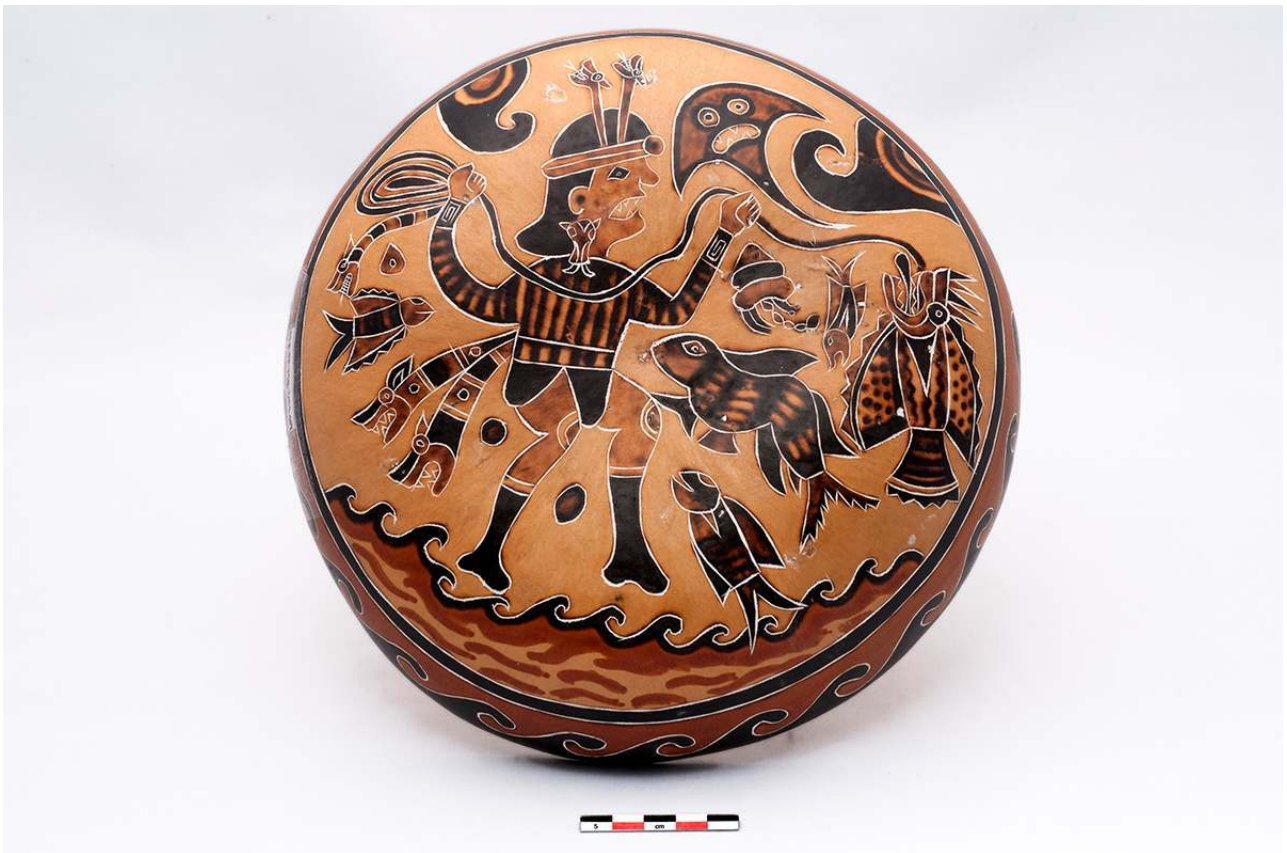

() J.C. Domenech

- Pérou ; province de Chiclayo, région de Lambayeque

- Représentation du dieu de la mer Aia-Paec, entouré de créatures marines sur une calebasse séchée (calabaza, Lagenaria vulgaris). Cette technique de gravure à l'aide d'un burin (mate burilado) a une antiquité de 3500 ans. Il s'agit ici d'une figure imaginée par l'artisan, librement inspirée des peintures mochicas. Objet décoratif

- 01/07/2009 ; collectrice Marina Quiñe

- Diamètre $20 \mathrm{~cm}$

- ETB-PE-MQ-2009-009

34) Ligne avec son plioir 


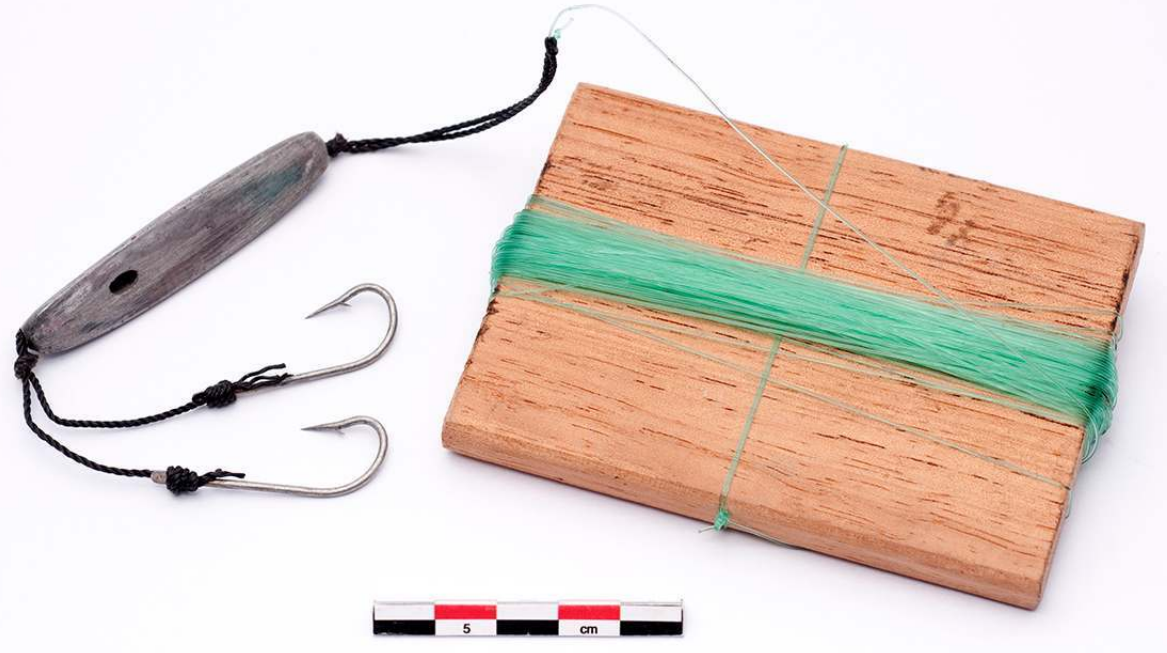

(C) J.C. Domenech

107 • Pérou, Huanchaco, département La Libertad; province Trujillo

- Linea de pesca con anzuelos y señuelo. Ligne avec son plioir

-20/08/2009; collectrice Marina Quiñe

- ETB-PE-MQ-2009-029

35) Hameçon et cuillère du Groenland

Figure 50 : Hameçon et cuillère (Groenland) MNHN-E-2016.6.23

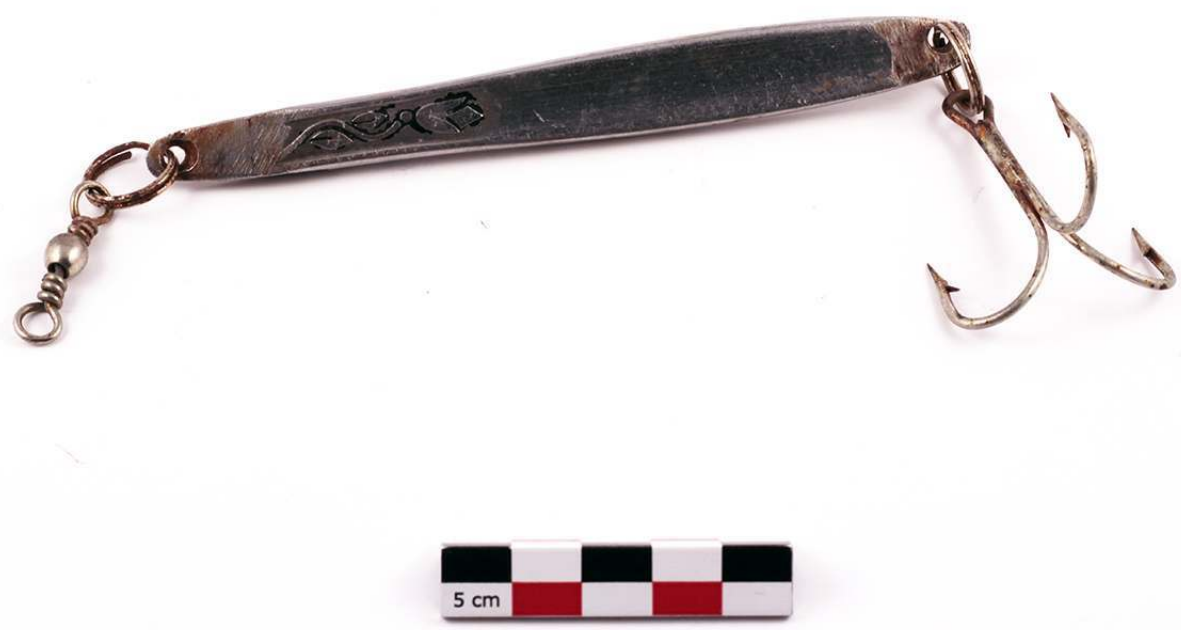

109 - Inuit. Groenland, Ammassalik, Tasiilaq

- Hameçon triple en grappin. La cuillère est faite avec un manche de couteau de table. Ce 
type d'hameçon est utilisé selon la technique de la « dandinette ", un mouvement de va et vient lui étant impulsé en agitant la ligne. L'émerillon à la base de l'hameçon imprime un mouvement rotatoire lorsque la ligne est traînée. On l'emploie en creusant un trou dans la glace sur les lacs gelés pour l'omble chevalier (Salvelinus alpinus, Salmonidés) ou de la banquise pour la morue (Gadus morrhua, Gadidés), dans des failles de la banquise pour le chabot (Myoxocephalus scorpius, Cottidés) ou enfin en kayak en eau libre toujours pour la morue. La pêche à la ligne aalisaat a été introduite par les Danois dans les années 1920 ; elle a donné son nom sur la côte Est à la morue aalisakkat « ceux qui se prennent à la ligne » (Robbe P. 1973 : 137-143)

- 2004 ; collecteurs Bernadette et Pierre Robbe

- Longueur totale $23 \mathrm{~cm}$; longueur de l'hameçon $5 \mathrm{~cm}$, largeur $4,5 \mathrm{~cm}$; longueur de la cuillère $13,5 \mathrm{~cm}$, largeur $1,6 \mathrm{~cm}$

- MNHN-E-2016.6.23

36) Hameçon et cuillère du Groenland

Figure 51 : Hameçon et cuillère (Groenland) MNHN-E-2016.6.89 (face et dos plombé)

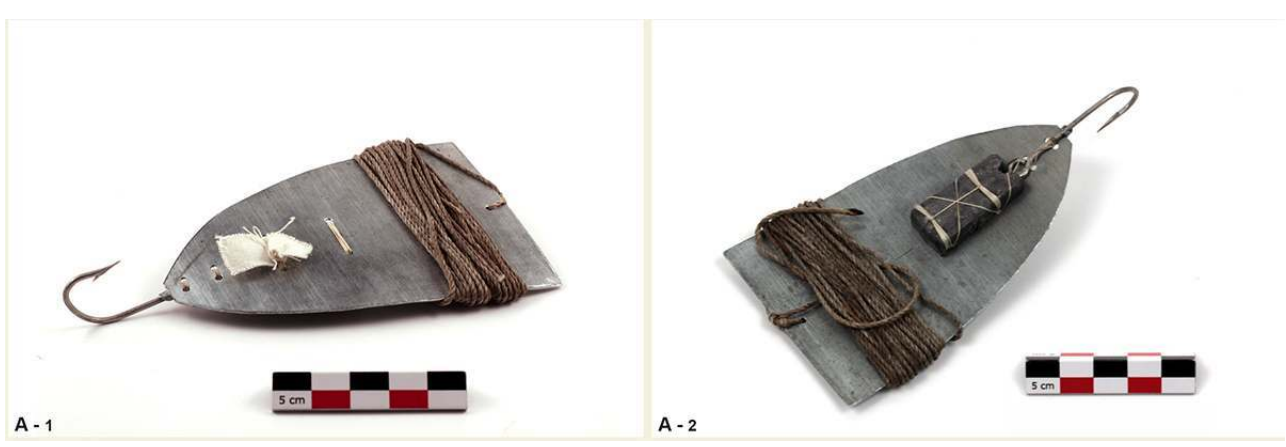

(c) S. Bahuchet

111 - Inuit. Groenland, Ammassalik, Tasiilaq

- Hameçon pour la pêche à la dandinette. L'hameçon est soigneusement cousu sur une plaque métallique rigide en aluminium servant de cuillère, à laquelle est également cousu un plomb rectangulaire de $3 \times 1,6 \mathrm{~cm}$, avec du fil métallique. Le tissu blanc, qui bouge dans l'eau, fait office de leurre. On trouvera un dessin d'enfant de cette pêche à la ligne dans Robbe B. (2010:33)

- 1979 ; collectrice Bernadette Robbe

- Longueur 14,4 cm ; largeur 7,5 cm ; longueur du hameçon 3,1 cm

- MNHN-E-2016.6.89 
Figure 52 : Pêcheurs d'ombles en pleine action dans le torrent de Koorngarmiit (Ammassalik, 2004)

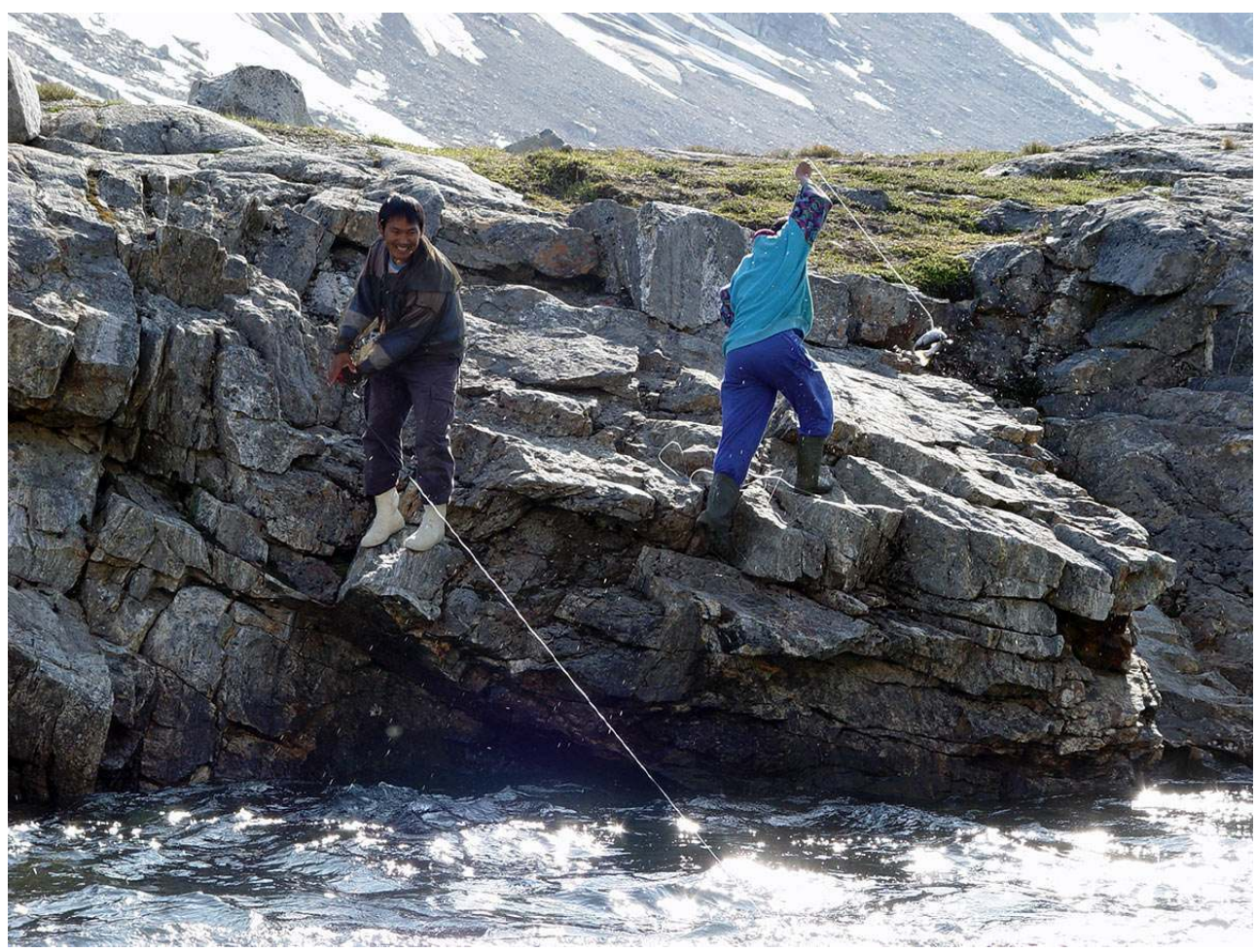

(c) P. Robbe

\section{2 - Pêche directe}

113 - Ligne à canne, flottante

114 37) Hameçons à congre, France

Figure 53 : Hameçons et bas de ligne ETB-FR-SB-2015-003

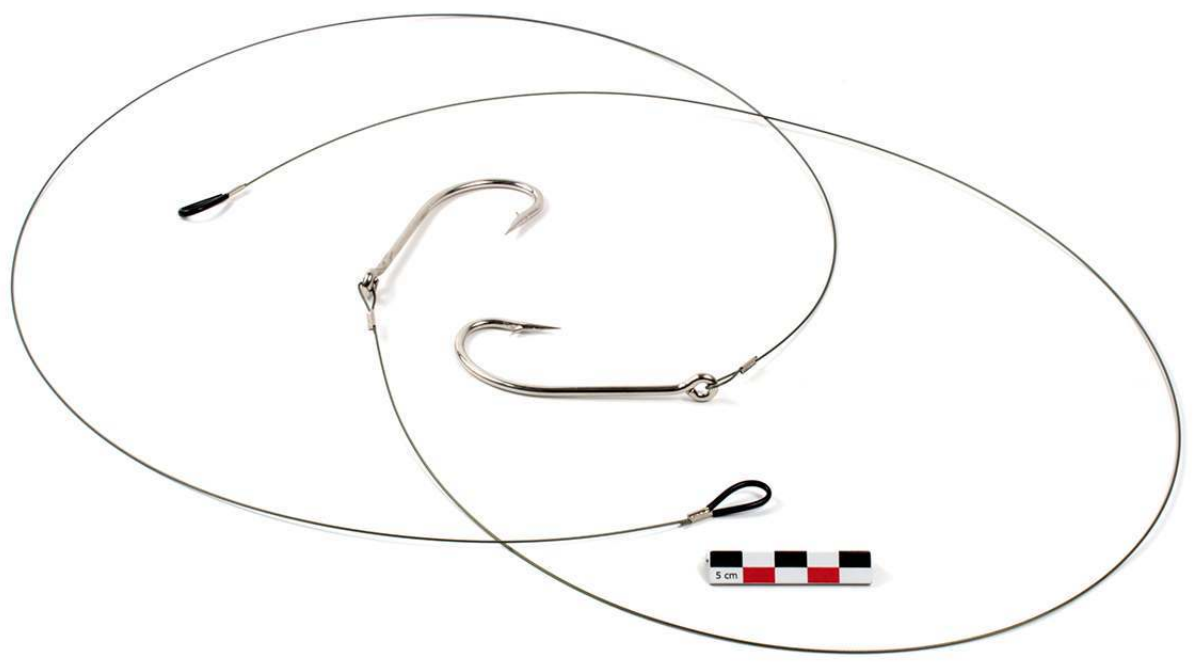

(c) P. Rameau 
115 • France ; Erquy, Côtes d'Armor ; Comptoir de la mer

- Pochette de 3 hameçons sur bas de ligne en métal. Marque Tortue

- 9/2016 ; collecteur Serge Bahuchet

- Hameçon 9,5 x 3,5 cm ; bas de ligne $90 \mathrm{~cm}$

- ETB-FR-SB-2016-003

116 38) Hameçon cuillère

Figure 54 : Hameçon cuillère ETB-FR-MC-2018-001

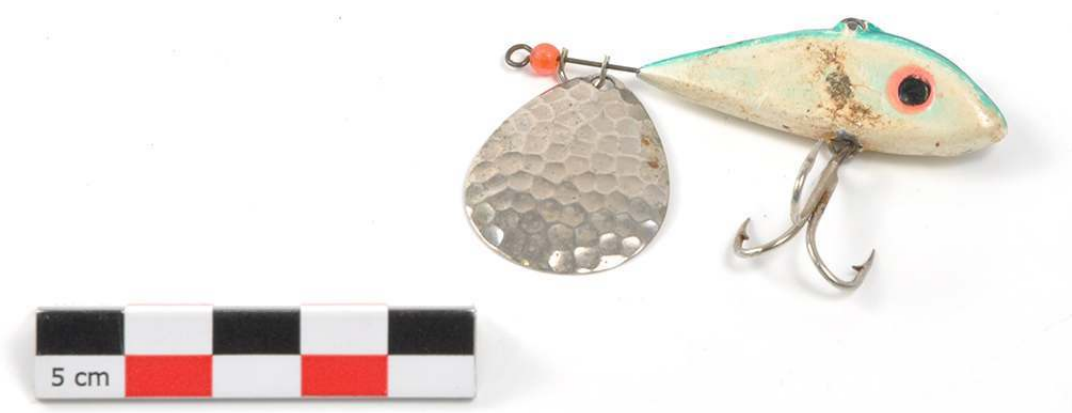

(c) P. Rameau

117 - France ; Carnac, Morbihan

- Hameçon d'une facture caractéristique des années 50. Appât en forme de poisson en métal peint ; cuillère métallique brillante suspendue à la queue ; hameçon triple. Destinée à la pêche à la traîne plusieurs hameçons (six, par exemple) sont accrochés sur une même ligne, traînée derrière le bateau, en suivant les bancs de maquereaux, souvent au soleil couchant. La cuillère maintient les hameçons entre deux eaux, tout en attirant les poissons. • Le pêcheur remonte sa ligne régulièrement

- Date de collecte ; collectrice Mélanie Congretel

- Longueur $6,5 \mathrm{~cm}$; poisson 4,5 x 1,5 cm ; cuillère $3,5 \times 2,5 \mathrm{~cm}$; hameçon $2 \times 1,5 \mathrm{~cm}$

- ETB-FR-MC-2018-001

39) Figurine chinoise d'un pêcheur à la ligne 
Figure 55 : Jouet-figurine représentant un pêcheur à la ligne MNHN-E-2011.9.52

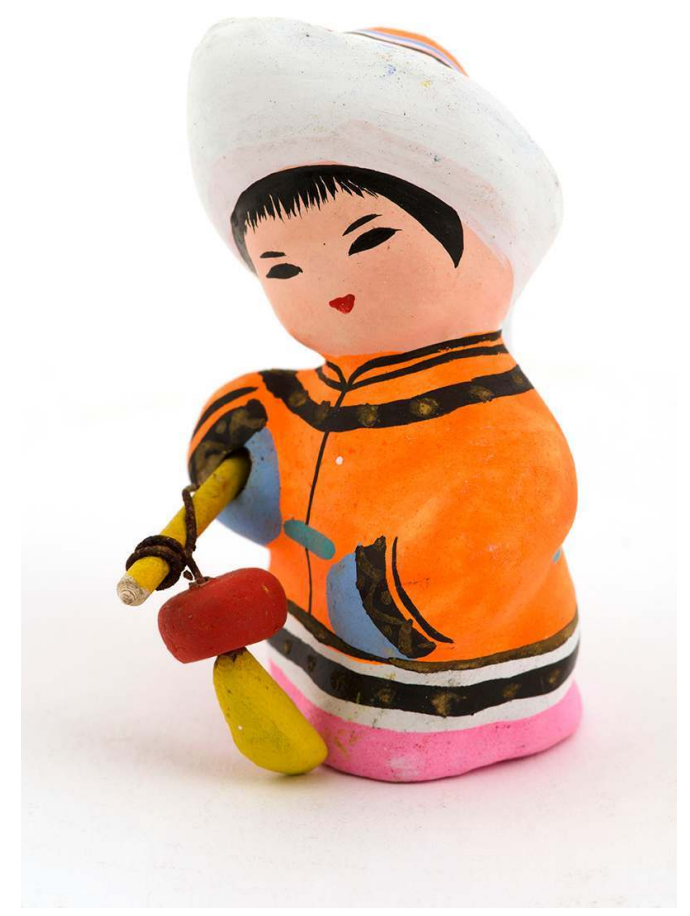

(c) J.C. Domenech

119 - Chine ; Province du Yunnan

- Figurine en argile peinte, partiellement recouverte de papier

- Collectes entre 1970 et 1985 ; collecteurs Jean-Louis Boissier et Liliane Terrier

- Hauteur totale 5,5 cm, largeur max. $4 \mathrm{~cm}$, profondeur max. $4 \mathrm{~cm}$

- MNHN-E-2011.9.52

120 40) Dessin d'enfant algérien 
Figure 56 : Dessin d'enfant MNHN-E-2013.8.136(2)

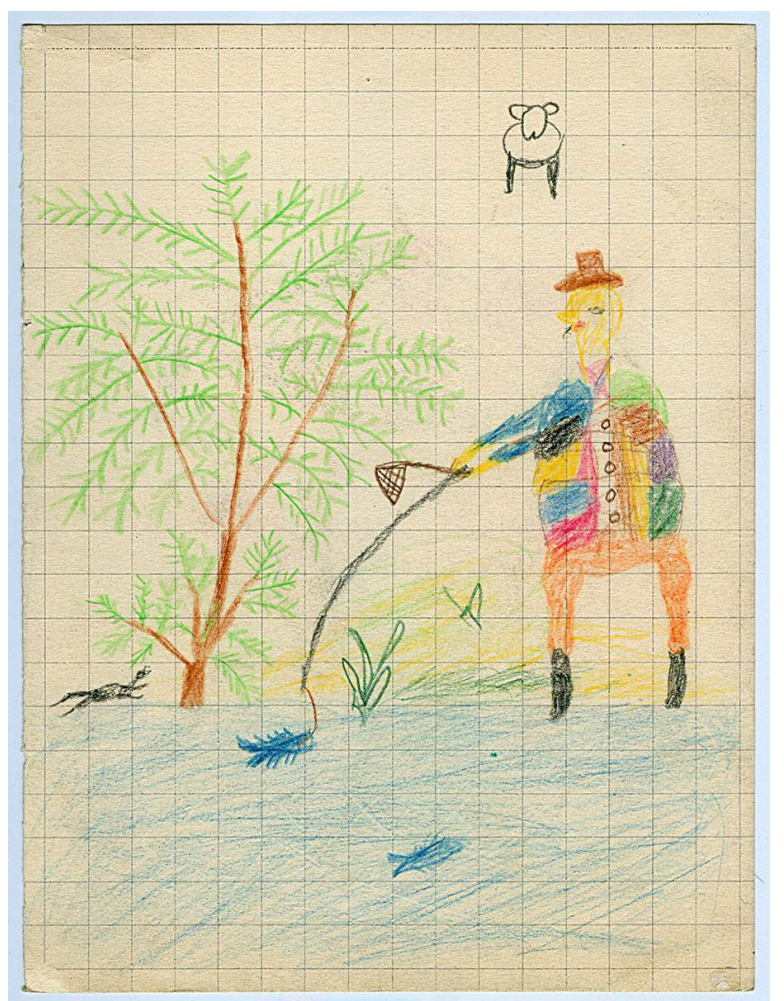

(c) D. Brabant

- Algérie ; Oasis de Tabelbala, région de Saoura

- Dessin d'enfant représentant une pêche à la ligne. Le pêcheur tient à la main droite une épuisette pour récupérer le poisson pris par l'hameçon

- Entre 1948 et 1961 ; collectrice D. Champault; ancienne collection du département d'Afrique du Nord

- Feuille 22 x $17 \mathrm{~cm}$ à carreaux de 1 x $1 \mathrm{~cm}$

- MNHN-E-2013.8.136(2)

Les flotteurs

Le flotteur ou bouchon, complété par les plombs, sert d'abord à régler la longueur à laquelle on veut que l'hameçon plonge, par rapport à la surface, au fond, ou entre les deux. Ensuite, il sert de repère au pêcheur pour déchiffrer le comportement du poisson, s'il s'approche de l'appât par le frémissement du flotteur, surveiller s'il a mordu lorsque le bouchon s'enfonce, et donc décider du mouvement pour accrocher la proie («ferrer ») et la sortir de l'eau.

41) Flotteurs de France à tube en plume d'oie 


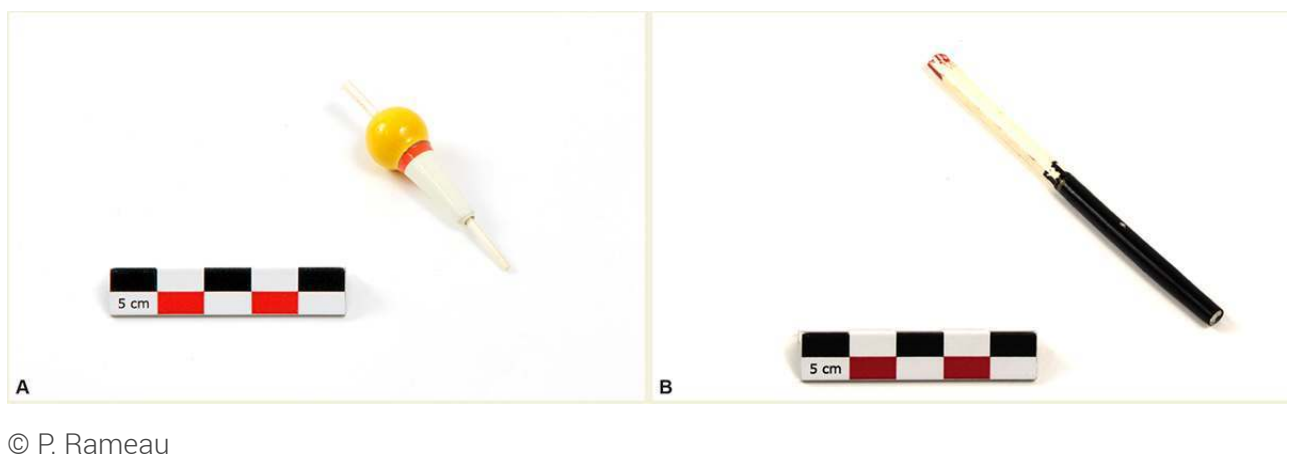

- ETB-FR-RP-2012-040: Bouchon flotteur pour ligne de pêche, vertical, monté sur un biseau de plume du bout de l'aile d'oie appelée « flèche » (réf. Lecuyer \& Pujol 1975)

- Années 1970 ; collecteur Raymond Pujol

- Longueur $6,2 \mathrm{~cm}$, diamètre $1,5 \mathrm{~cm}$

- ETB-FR-RP-2012-041 : Flotteur léger en tuyau de plume d'oie simple, aux extrémités bouchées ; la teinte rouge de l'extrémité supérieure permet de le voir à la surface de l'eau.

- Montchanin, Saône et Loire

-4/3/1977 ; collecteur Raymond Pujol

- Longueur $9,8 \mathrm{~cm}$, diamètre $0,5 \mathrm{~cm}$

42) Trois flotteurs en piquants de porc-épic

Figure 58 : Flotteurs en piquants de porc-épic ETB-FR-RP-2012-055

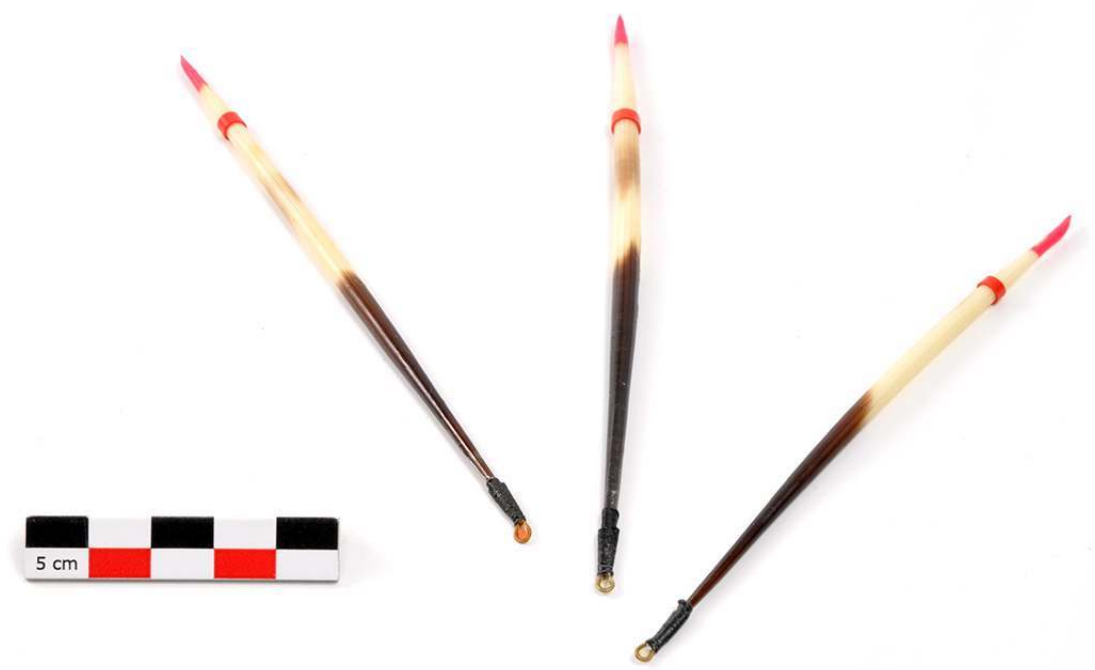

(C) P. Rameau

127 - France

- Trois flotteurs légers («plumes») en piquants de porc-épic d'Afrique centrale (Atherurus africanus), chacun terminé par une boucle métallique. Ces flotteurs étaient réputés car très sensibles à la touche du poisson, dans les eaux tranquilles, et profondes. La ligne passe dans l'anneau et dans la ligature rouge de l'extrémité. On trouve ce type de «plume» en vente dans les catalogues successifs de la Manufacture des armes et des 
cycles de Saint Etienne dès 1912

- Années 70 ; collecteur Raymond Pujol

- Longueur 13 et $14 \mathrm{~cm}$

-ETB-FR-RP-2012-055
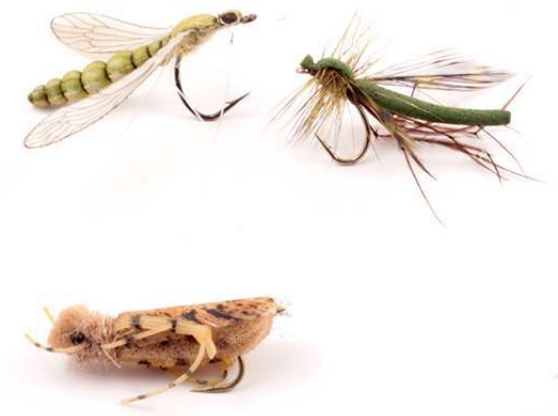

Ligne à canne, lancée entomologiste. fonction de la saison.

\section{3) Lot de 9 mouches pour truite d'eau douce}

Figure 59 : Lot de mouches pour la truite

Cette technique connue sous le nom de pêche au lancé, ou encore de pêche à la mouche, consiste à «faire sautiller l'hain à la surface de l'eau » (entendez l'hameçon, selon la terminologie moderne) pour reprendre l'expression de Duhamel du Monceau. Pour faire affleurer l'appât, il ne faut ni plomb, ni flotteur, et un habile coup de main pour imiter le vol des insectes. Cette technique est destinée aux petits poissons de surface. Pour remplacer les insectes vivants réels, on confectionne des mouches artificielles, avec des fragments de poils et de plumes. Duhamel du Monceau rapporte que ce sont les pêcheurs anglais qui ont inventé cette pratique, qui s'introduisit en France de son temps, au milieu du XVIII ${ }^{e}$ siècle. Les «mouches » imitent la forme d'insectes, mais Duhamel insiste sur le fait que l'on ne saurait reconnaître exactement des espèces réelles, tel que le ferait un

Néanmoins le montage des mouches relève d'un profond savoir-faire et s'appuie sur des observations fines de l'environnement. C'est non seulement la connaissance du comportement du poisson et de son alimentation mais, plus généralement, aussi celle de l'écosystème associé au lieu de pêche qui se reflète dans le choix de cet objet matériel qu'est la mouche de pêche. En effet, les pêcheurs vont adapter le choix du leurre en
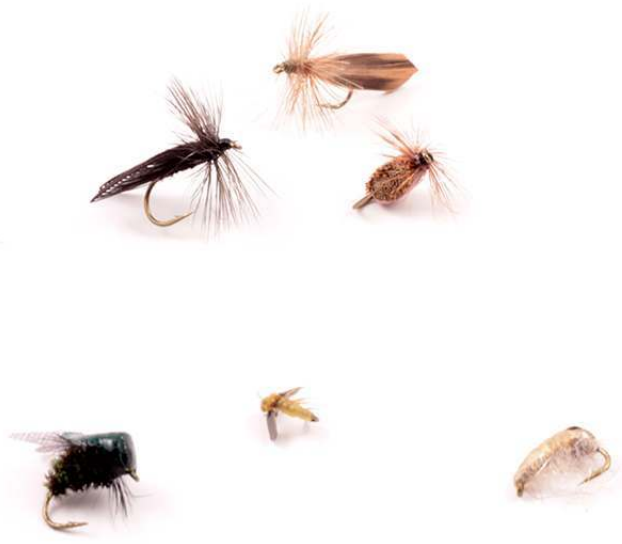

(c) S. Bahuchet

132 - France. Paris; magasin spécialisé La Maison de la mouche-Dubos

- Cet ensemble de mouches est destiné à la pêche au lancer en eau douce (rivière et étang notamment) pour la recherche des truites. La qualité de ces leurres tient à la finesse de leur ressemblance avec les insectes présents habituellement dans la nature. Ce lot comprend : deux cousins, une sauterelle, trois papillons (dont un au stade émergent), une mouche à viande, un scarabée et un gammare. L'imitation de chaque grande famille 
scientifique d'insecte est désignée, dans la terminologie de la pêche à la mouche, par un mot anglais. Par exemple les " papillons » (en réalité des trichoptères) sont appelés sedge. Ces leurres de pêche sont entièrement confectionnés à la main. Certaines de ces mouches de pêche sont montées à partir de matières naturelles. Par exemple le flan du papillon noir est composé de plumes de cane, tandis que sa crête est faite de plume de dinde. Mais d'autres types de plumes et de poils peuvent être utilisés : plume de coq, de canard, poil de lapin, de lièvre, etc. Depuis une vingtaine d'années, les leurres viennent à être aussi confectionnés en matière synthétique. Ce lot a été acquis auprès de la Maison de la moucheDubos

- 2014 et 2015 ; collectrice Nastassia Reyes

- Dimensions du plus petit 8 × $5 \mathrm{~mm}$, du plus grand $31 \times 35 \mathrm{~mm}$

- ETB-FR-NR-2015-001

\section{4) Mouche pour pêche en mer}

Figure 60 : Mouche pour poissons de mer

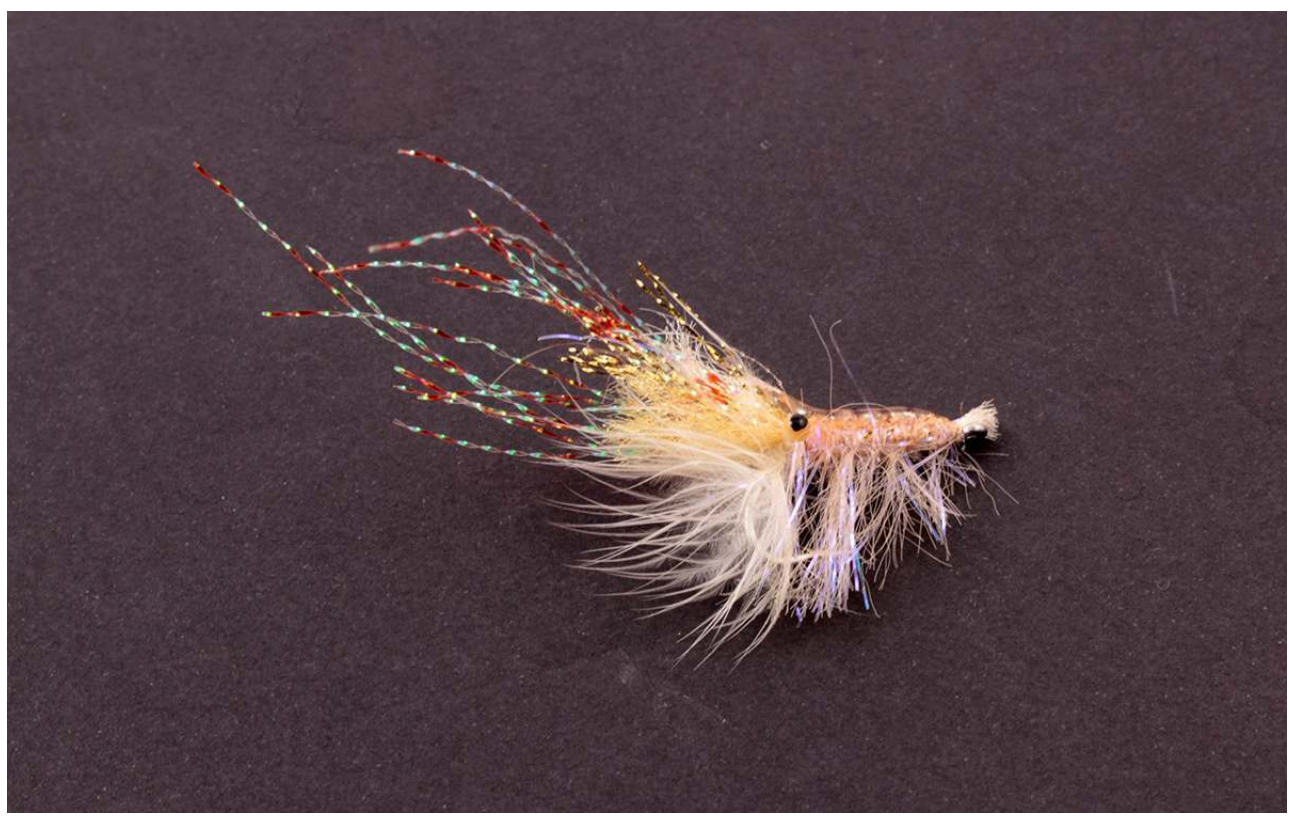

(c) S. Bahuchet

- France. Paris ; magasin spécialisé La Maison de la mouche-Dubos

- Mouche en forme de crevette, destinée à la pêche au lancer en mer du saumon ou du bar

- 2015 ; collectrice Nastassia Reyes

- Longueur du corps $3 \mathrm{~cm}$, longueur totale avec les antennes $7 \mathrm{~cm}$

- ETB-FR-NR-2015-002

- Ligne flottante

45) Ligne flottante de l'Orénoque 
Figure 61 : ligne flottante MNHN-E-2012.7.7 (entière et détail de la ligne)

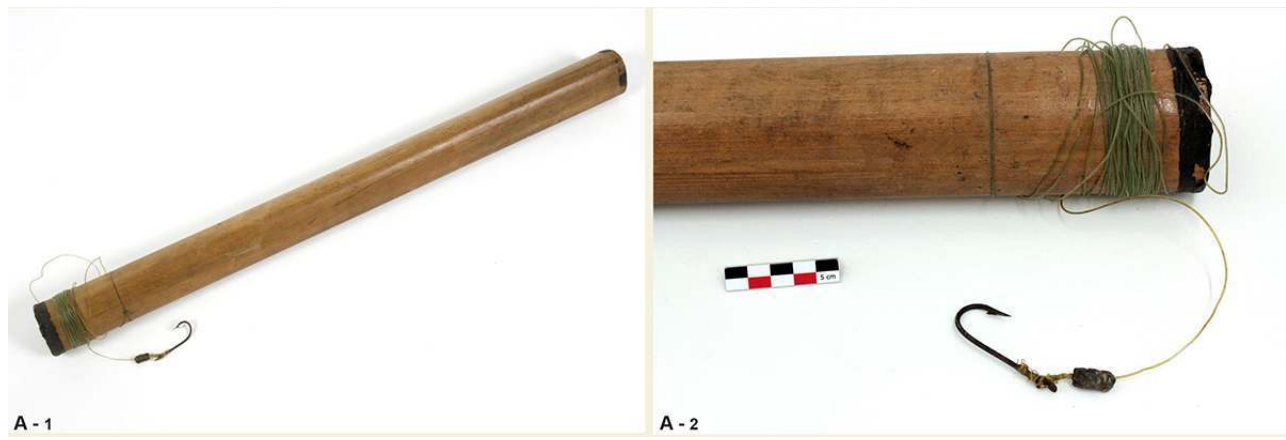

(c) A-1 : J.C. Domenech; - 2 : S. Bahuchet

137 - Ethnie Warao. Venezuela ; Delta de l'Orénoque

- Flotteur de pêche en rachis de palme (Mauritia flexuosa ; cf. Suarez 1966 : 37) avec ligne en nylon et hameçon en fer, lesté d'un plomb. Le fil étant dévidé et appâté, le flotteur est laissé à flotter à la surface du fleuve, en dérivant. De nombreuses lignes sont disposées en même temps ; le pêcheur, de sa pirogue, navigue en surveillant les mouvements :

«La pêche avec des flotteurs consiste à disposer plusieurs engins dans un lieu aux eaux calmes. Le pêcheur, à l'ombre des palétuviers au bord de l'eau, se laisse dériver dans le courant, tout en surveillant d'un œil vigilant le va et vient des flotteurs. Des mouvements légers indiquent qu'un poisson a mordu ; aussitôt il récupère sa proie et l'assomme à coup de bâton. " (Suarez 1968: 81)

- 1974 ; collectrice Brigitte Gladu

- Longueur 79,5 cm, diamètre7 cm. Taille de l'hameçon longueur $6 \mathrm{~cm}$, largeur $2,2 \mathrm{~cm}$

- MNHN-E-2012.7.7

- Techniques indirectes

- Ligne calée, palangre

46) Hameçon pour palangre de fond 
Figure 62 : Hameçon japonais pour palangre ETB-JP-SB-2018-002

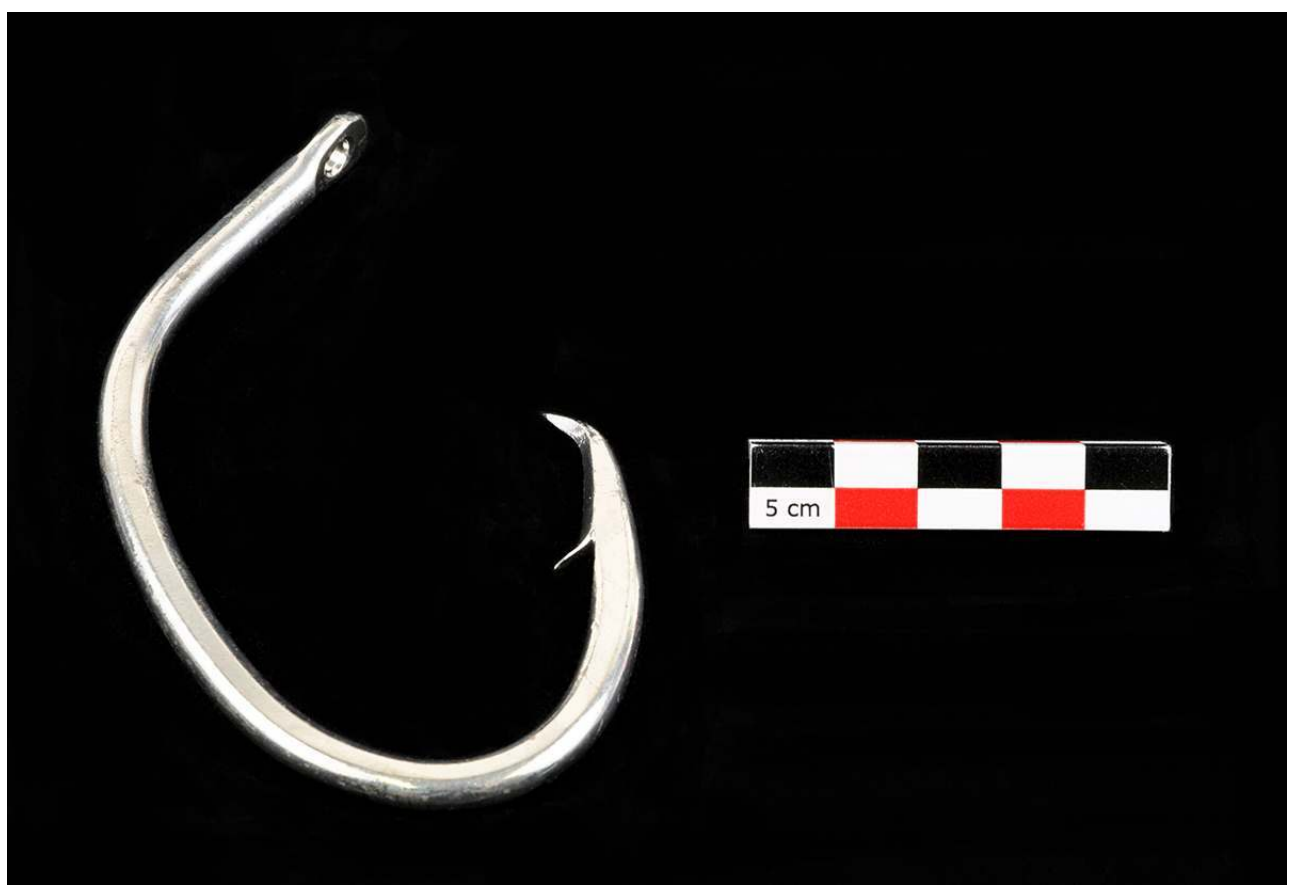

(C) P. Rameau

141 - Japon ; Toba, Préfecture de Mie

- Hameçon pour palangre de fond, pour le Scombrops boops (mutsu), Scombropidés (Scombre; Gnomefish; Japanese blue-fish). Ce poisson assez grand (de $50 \mathrm{~cm}$ à $1,5 \mathrm{~m}$ de longueur) est présent dans tout le Japon et l'est de la mer de Chine, dans les fonds rocheux, à une profondeur de 200 à $700 \mathrm{~m}$

- 1994 ; collecteur Serge Bahuchet

$\cdot 11,5 \times 7 \mathrm{~cm}$

- ETB-JP-SB-2018-002

47) Panier et palangre de Sicile

Figure 63 : Palangre de Sicile dans son panier ETB-IT-NR-2015-003 (vue d'ensemble et détail des hameçons accrochés au bord du panier)
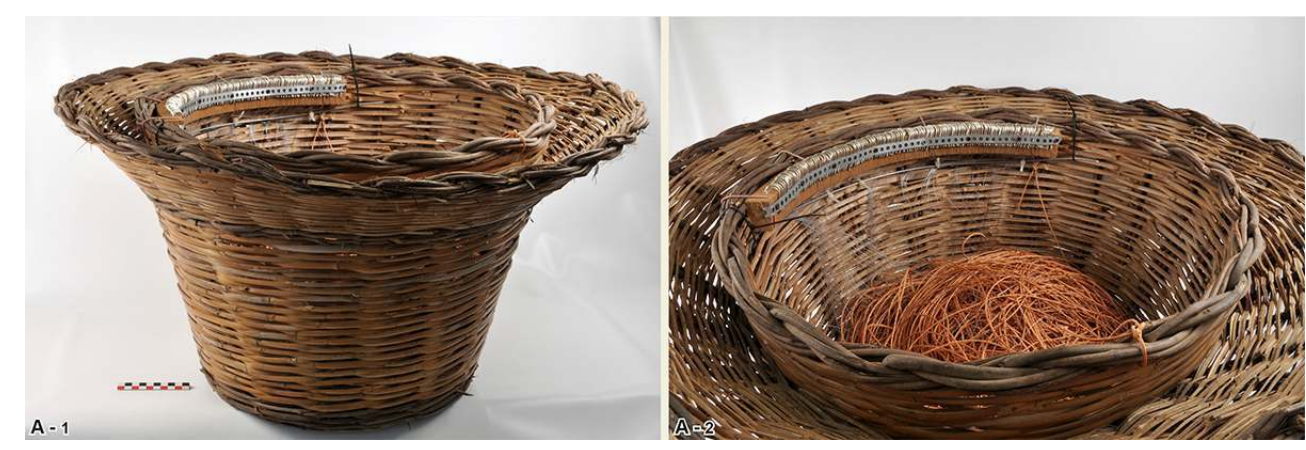

(C) P. Rameau

$143 \cdot$ Italie ; Catane, Sicile

- Cet objet est un engin de pêche employé naguère par les pêcheurs à la piccola pesca de Sicile. Le panier (coffa en italien) est construit à partir de l'entrelacement des roseaux. Il 
est typique des années 1940-1960 (avant l'usage des réservoirs en plastique). Il contient une palangre de 160 hameçons, désignée en italien conzo, également coffa ou palangito. Une palangre est composée d'une ligne principale («ligne-mère») qui peut être constituée d'une corde, d'un monofilament ou d'un fil d'acier. Des lignes secondaires (les avançons) pourvues d'hameçons sont fixées sur la ligne-mère à intervalles réguliers (Fig. 64B). Les lignes de cet exemplaire sont en tresse synthétique. Cet objet a été acquis auprès de Davide Aggnino, pêcheur de Catane. Il était utilisé par son père. Il a été, ensuite, restauré par Aggostino Calogero. Les hameçons ont été fixés au panier par une plaque métallique pour les besoins de l'entreposage dans le musée, pour éviter des accidents de manipulation

- 2015 ; collectrice Nastassia Reyes

- Diamètre $76 \mathrm{~cm}$, hauteur $40 \mathrm{~cm}$; hameçons 3,5 x 1,5 cm

- ETB-IT-NR-2015-00

Sur la côte ionienne de la Sicile, avant l'utilisation du monofilament, la ligne-mère de la palangre était jusqu'au début des années 60 , préparée à l'aide de cordes torsadées ou tressées auxquelles étaient reliés les avançons formés par du crin de cheval entrelacé. De nos jours, la palangre est utilisée comme un outil de pêche de surface et de fond. En fonction de la taille des hameçons utilisés, de l'épaisseur de la ligne-mère ainsi que de l'épaisseur des avançons, cet outil peut permettre la capture d'espèces pélagiques (thons, espadons, daurade coryphènes) mais aussi de poissons benthiques et démersaux, vivant proche $\mathrm{du}$ fond (merlus, dentis, rascasses, congres). Pour la capture des espèces pélagiques, la ligne-mère est équipée de flotteurs, ce qui maintient la palangre en surface, entre-deux eaux ou encore portée par les courants marins; dans ce dernier cas elle est désignée comme palangre dérivante ou semi-pélagique. Enfin, munie de poids, la palangre reste encrée dans le fond et vise la capture d'autres espèces. Cet engin est donc, selon le montage, utilisé tant dans la pêche artisanale que dans la pêche hauturière (Reyes $2017: 235$ ). 
Figure 64 : A) Préparation des palangres. Les flotteurs jaunes indiquent qu'il s'agit d'une palangre de surface. On distingue les sardines-appâts. B) schémas de la structure et de la disposition des palangres.

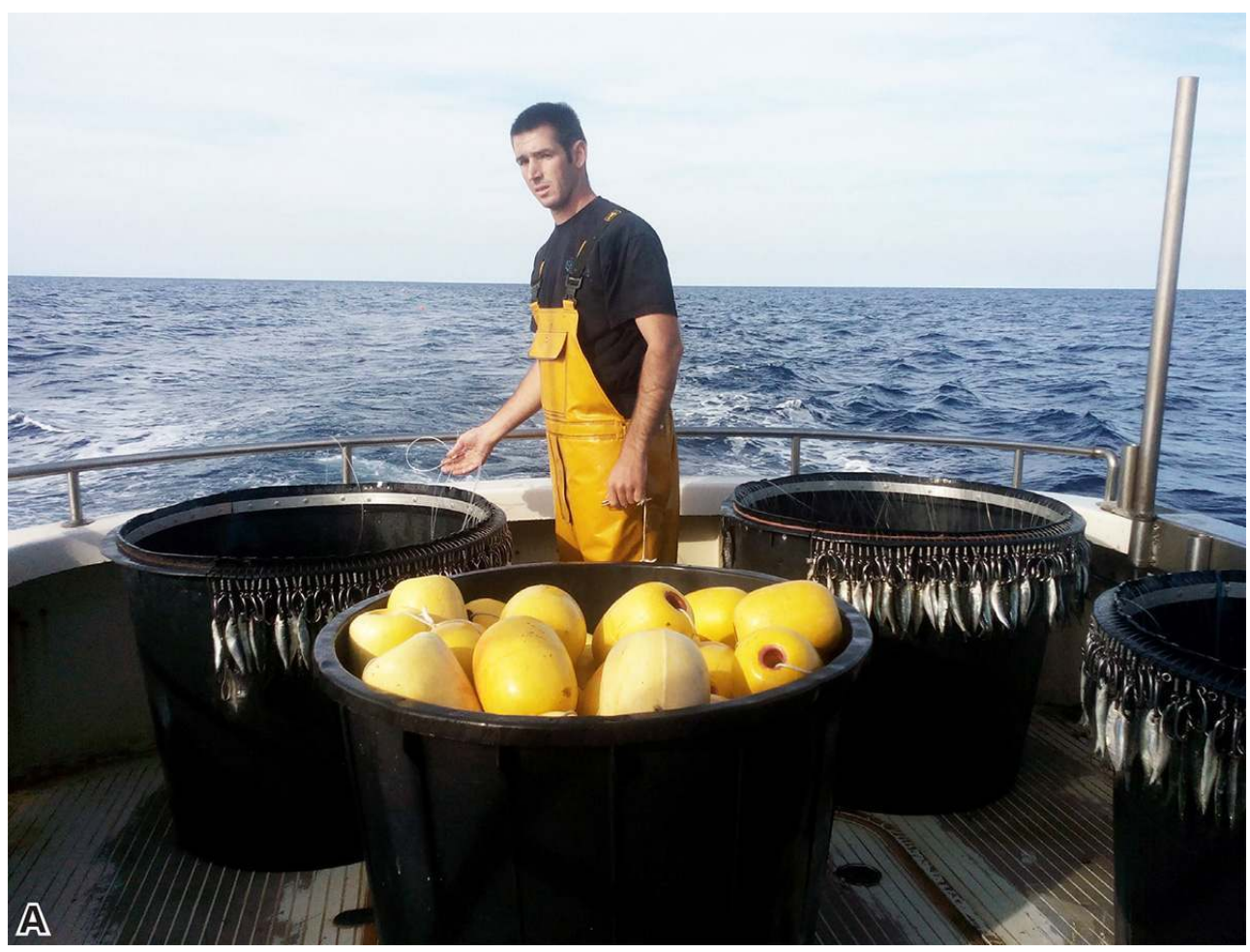

(C) N. Reyes
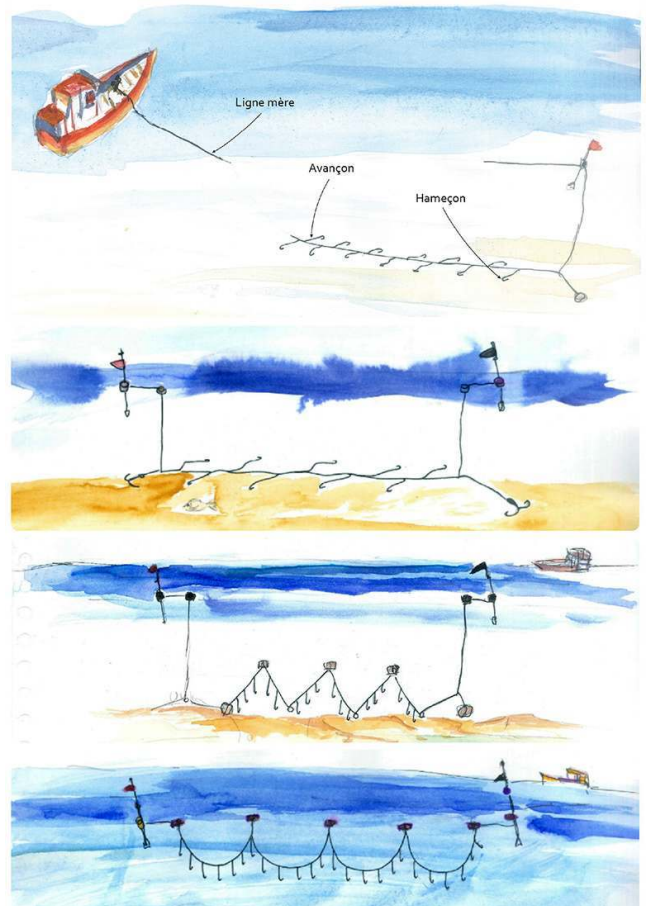

(C) N. Reyes 


\section{Auto-accrochage (embrouillage, coincement) (Classe V)}

\section{8) Pot à poulpe de Provence}

Figure 65 : Pot à poulpe de Provence ETB-FR-SB-2018-005

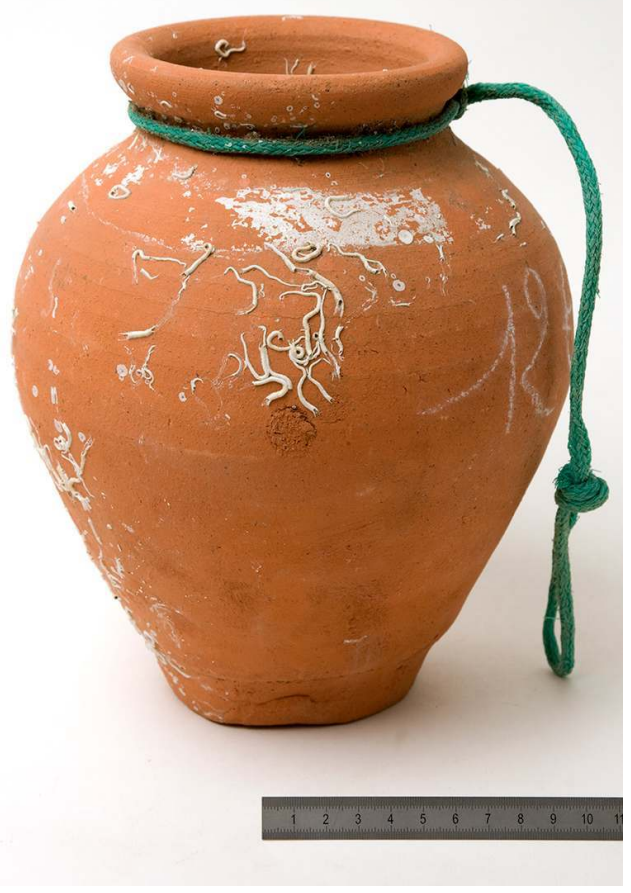

(C) J.-C. DOMENECH

147 - France, Provence (achat en brocante)

- Pot en céramique tournée, disposé en chaînes sur les fonds sableux, dans lequel les poulpes se réfugient. Lorsque le pêcheur remonte sa ligne de pièges, les poulpes ne s'échappent pas mais s'accrochent aux parois du vase

- Années 2000 ; collecteur Serge Bahuchet

- Hauteur $24 \mathrm{~cm}$, diamètre $16 \mathrm{~cm}$

- ETB-FR-SB-2018-005

\section{Accrochage provoqué, (Classe VI)}

T. Monod (1973 : 216) avance avec prudence la proposition d'une classe destinée « un mode d'action impliquant l'extraction de l'animal de son milieu ", qui à ce stade comporte très peu d'engins, dont le crochet pour déloger Cancer pagurus de son trou. Nous aurions 
quant à nous plutôt inscrit cette action dans la classe XII « Déterrement-ramassage ", mais il est vrai que dans ce cas, l'animal réagit directement à l'outil.

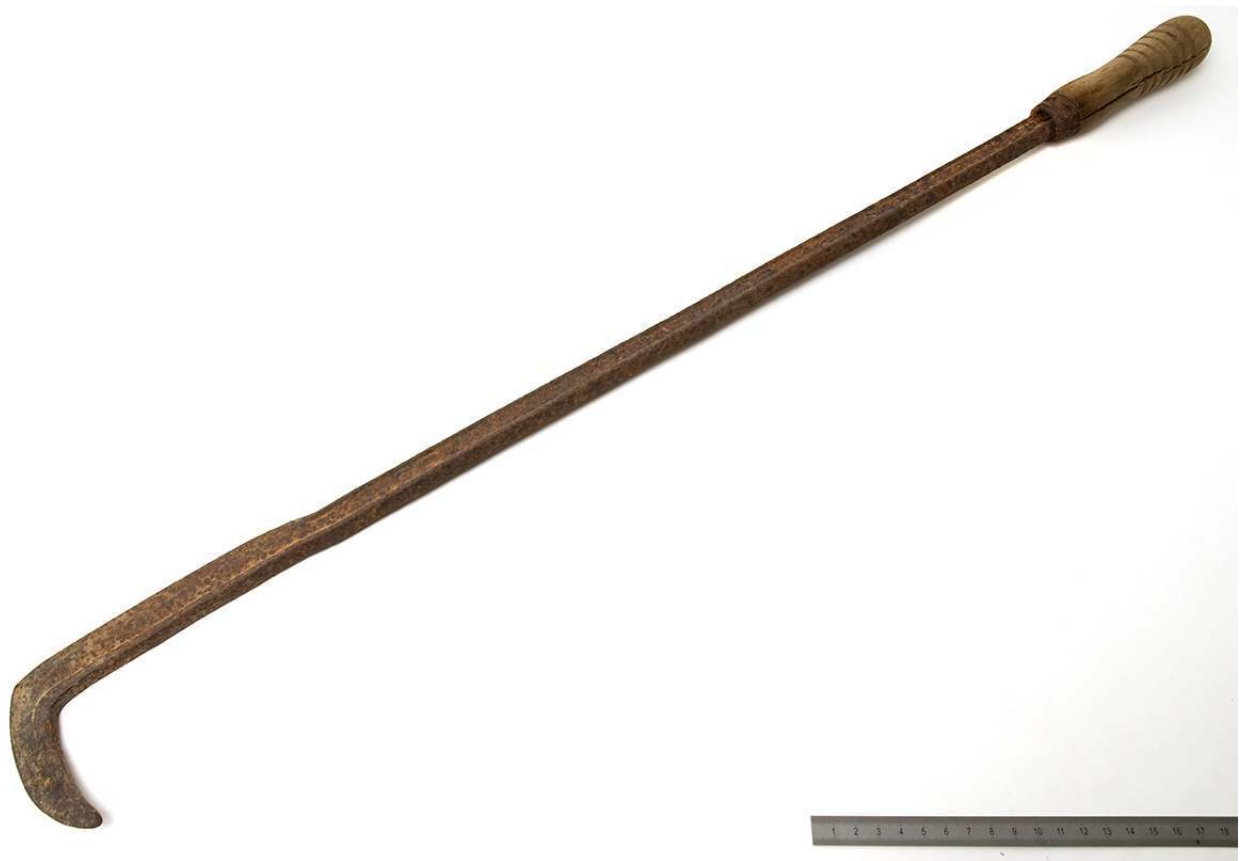

\section{(C) J.-C. Domenech}

- France ; La Tranche sur mer, Vendée

- Grand crochet en métal forgé et manche en bois. Objet ancien. Crochet utilisé pour pêcher les tourteaux et étrilles, auquel ils s'accrochent avec leurs pinces

- Années 1970 ; collecteur Raymond Pujol

- $81 \times 10 \mathrm{~cm}$

- ETB-FR-RP-2011-046

\section{Engins à parois immobilisantes (Cl. VIII)}

151 On distingue dans cette classe les engins actifs (actionnés par le pêcheur), selon l'angle de leur mouvement, des engins passifs, disposés dans l'eau et dans lequel les proies sont maintenues et emprisonnées.

\section{Engin actif, actionné en poussant ou levant}

\section{Recouvrement}

Les engins de ce groupe sont manipulés de haut en bas par le pêcheur, sur la proie. Ils peuvent être projetés et dans ce cas l'engin principal est le filet épervier $\left(n^{\circ} 48\right)$. Le pêcheur lance une nappe conique lestée sur l'eau, emprisonnant les poissons lorsque le filet s'enfonce dans l'eau. 
153 Ce procédé est très largement diffusé sur tous les continents, employé le plus souvent dans les cours d'eau intérieurs; il est singulièrement efficace dans les eaux turbulentes notamment dans les rapides.

154 Les engins peuvent être plongés dans l'eau, tenus à la main, et c'est le cas des panierscloches, fréquents en Afrique comme en Asie ( $\left.n^{\circ} 49\right)$. Généralement ces engins s'emploient collectivement, dans des eaux de faible profondeur ; une ligne de pêcheurs, hommes ou femmes, avancent de front, plongeant à chaque pas une paire de panier devant eux, coiffant ainsi les poissons trop lents (voir Figure 78).

50) Épervier

Figure 67 : Épervier ETB-FR-RP-2011-072

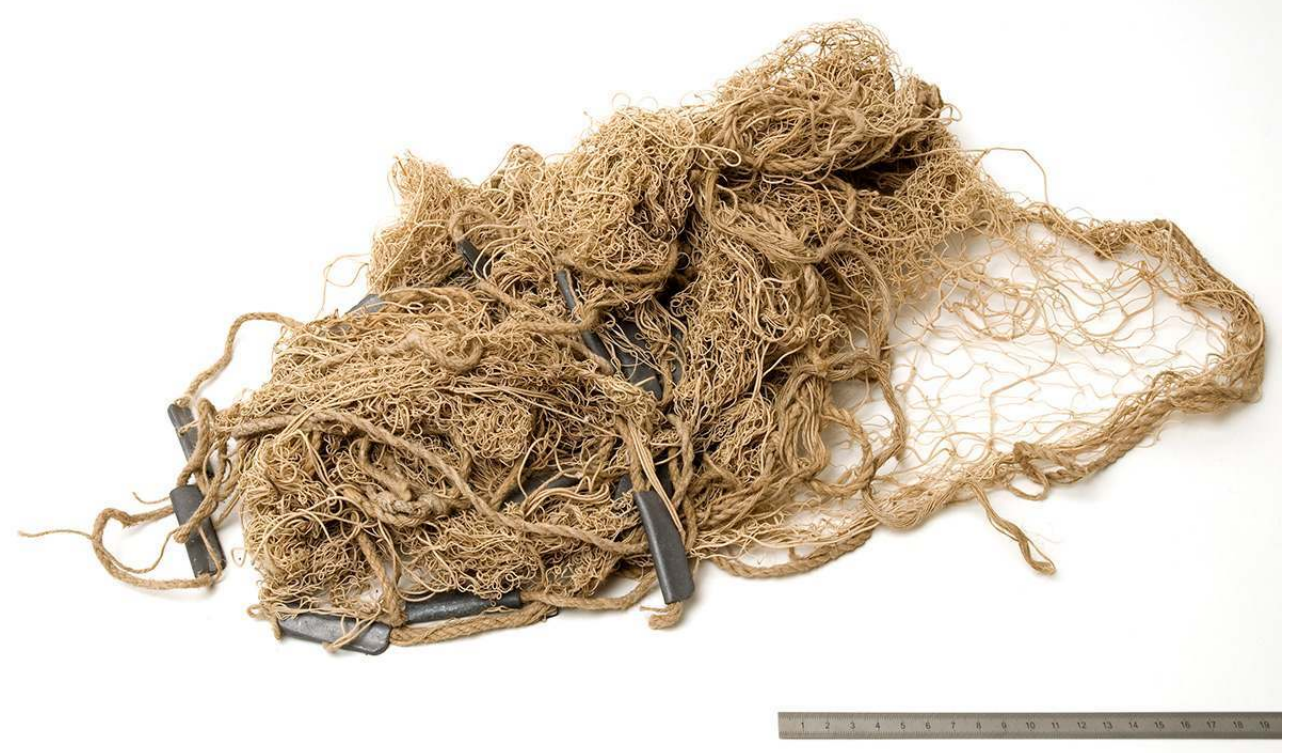

(c) J.C. Domenech

156 - France; Vendée (?).

- Débris d'un épervier, lesté de poids de plomb.

- Années 1970 ; collecteur Raymond Pujol

- ETB-FR-RP-2011-072 
Figure 68 : L'épervier vu par Duhamel du Monceau ( $2^{\mathrm{e}}$ section, pl. VII)

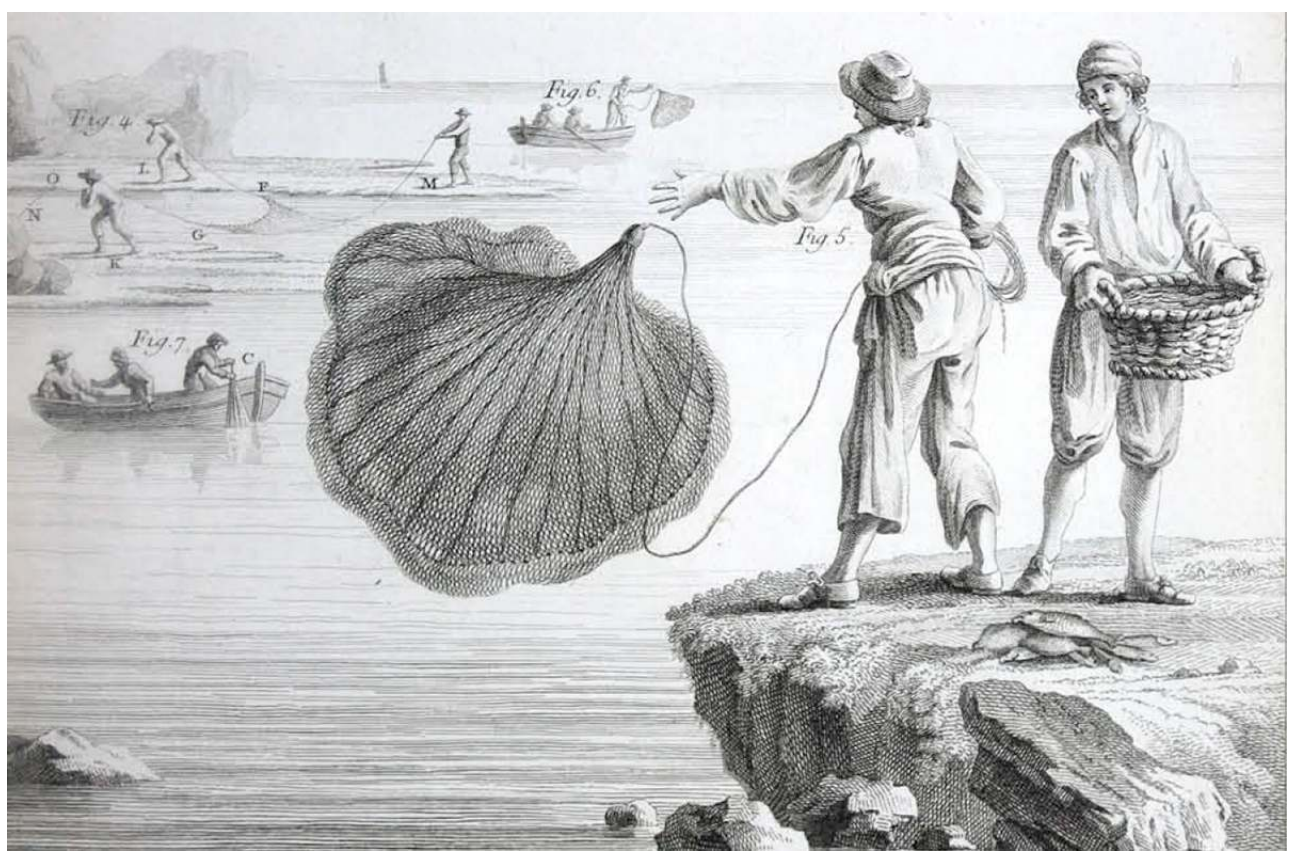

157 51) Panier-cloche du Bénin

Figure 69 : Panier-cloche du Bénin MNHN-E-2009.19.101

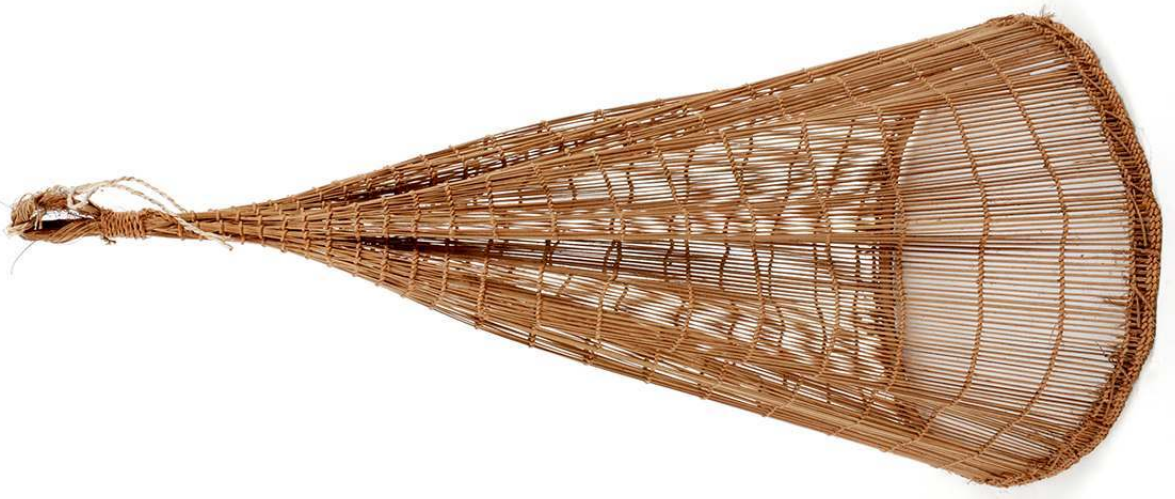

(C) J.C. Domenech

158 • Bénin ; $20 \mathrm{~km}$ au sud d'Abomey, département de Zou

- Panier de pêche, «panier-cloche »

-1980-1990 ; collecteur André Chauvin

- Longueur $105 \mathrm{~cm}$, diamètre $50 \mathrm{~cm}$

- MNHN-E-2009.19.101 


\section{Levée verticale ou oblique}

Figure 70 : Épuisette ETB-BR-SB-2009-010

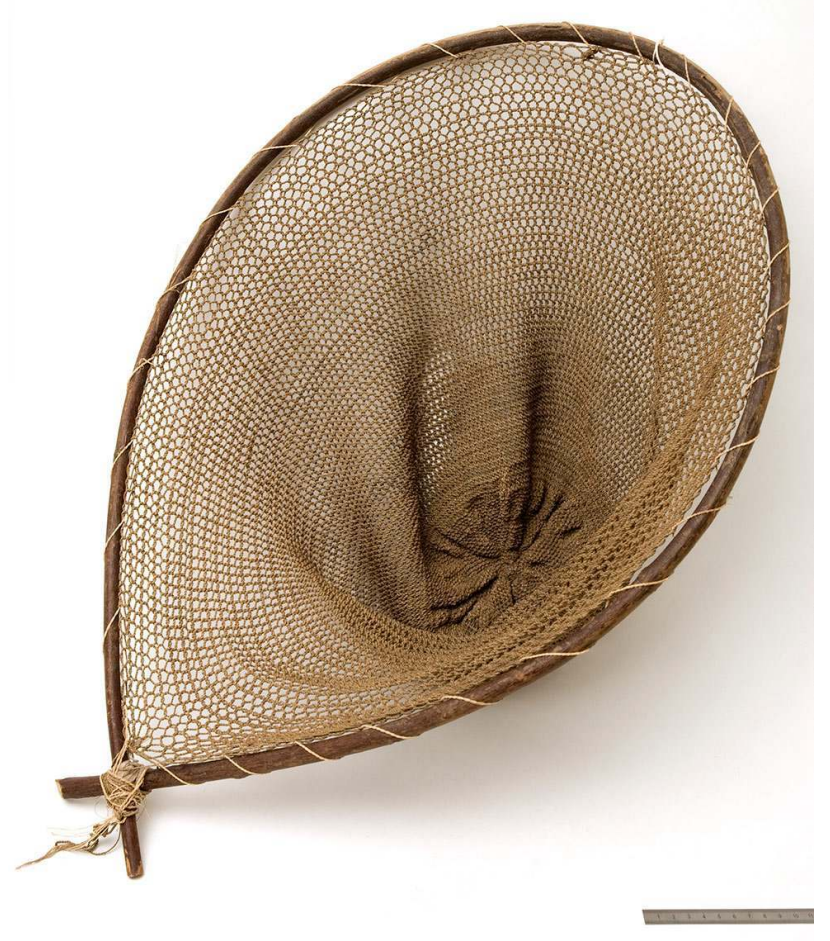

(c) J.-C. Domenech

160 -Ethnie Ikpeng. Brésil ; Région du Xingu ; achat FUNAI, Brasilia

- Épuisette, filet sur cadre puçà. Pêche en rivière pour petits poissons

- 2009 ; collecteur Serge Bahuchet

- $68 \times 46 \mathrm{~cm}$

- ETB-BR-SB-2009-010

161 53) Épuisette, filet sur cadre de Papouasie-Nouvelle Guinée 


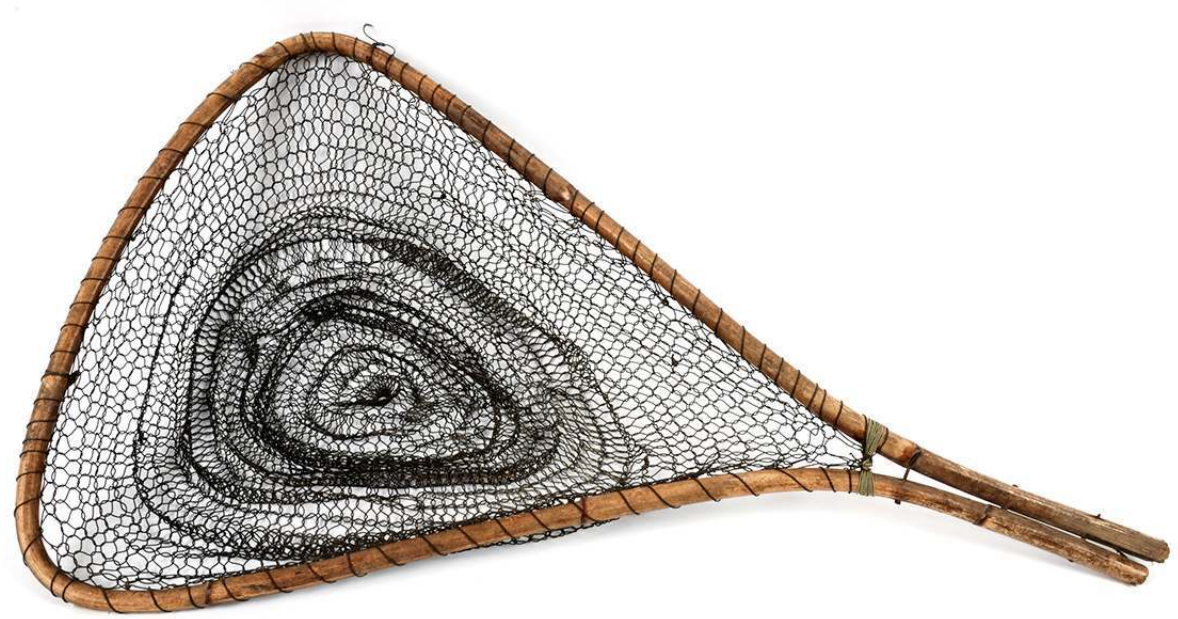

(C) J.C. DOMENECH

162 • Ethnie Mundugomor. Papouasie-Nouvelle Guinée; village de Kinakatem, région de Biwat, province du Sépik Est

- Épuisette ; filet noir fixé sur un cerclage de rotin, utilisé pour la pêche à pied

- Entre 2003 et 2005 ; collecteur Christian Coiffier

- Longueur $80 \mathrm{~cm}$, largeur $47,5 \mathrm{~cm}$, profondeur du filet $40 \mathrm{~cm}$

- MNHN-E-2006.1.28

54) Piège à crabes,

Figure 72 : Piège à crabe du Pérou ETB-PE-MQ-2009-026

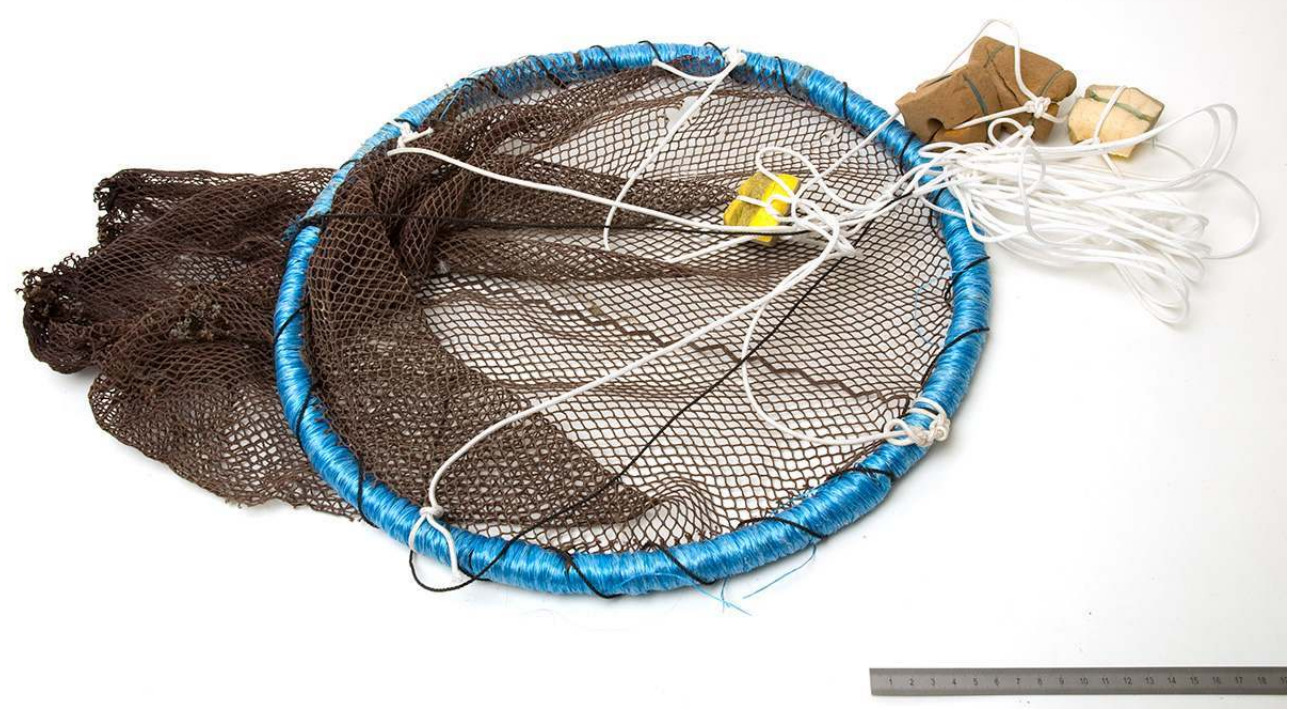

(c) J.C. Domenech 
- Pérou, Huanchaco, département La Libertad ; province Trujillo

- Saca. Piège à crabes fait par les pêcheurs. Sur un lourd cercle de fer, est fixé un sac fait en filet de pêche de fil synthétique. Pour pêcher des crabes, chaque pêcheur transporte 6 pièges dans son caballito de totora. À l'intérieur de chaque piège, il met un appât (têtes et viscères de poissons) et des roches pour faire du poids. Dans un trajet droit, il commence à déposer les pièges l'un après l'autre, que les poids retiennent au fond, à plat. Des flotteurs les relient à la surface, permettant de les retrouver et de les relever. Quand le pêcheur a fini de tous les déposer, il revient au début, pour relever le premier piège, qui est censé avoir déjà attiré des crabes. Ces filets sur cadre sont levés verticalement

-15/08/2009 ; collectrice Marina Quiñe

- Diamètre $40 \mathrm{~cm}$

- ETB-PE-MQ-2009-026

Figure 73 : Les filets à crabes séchant sur la grève, au pied des CABALLITOS

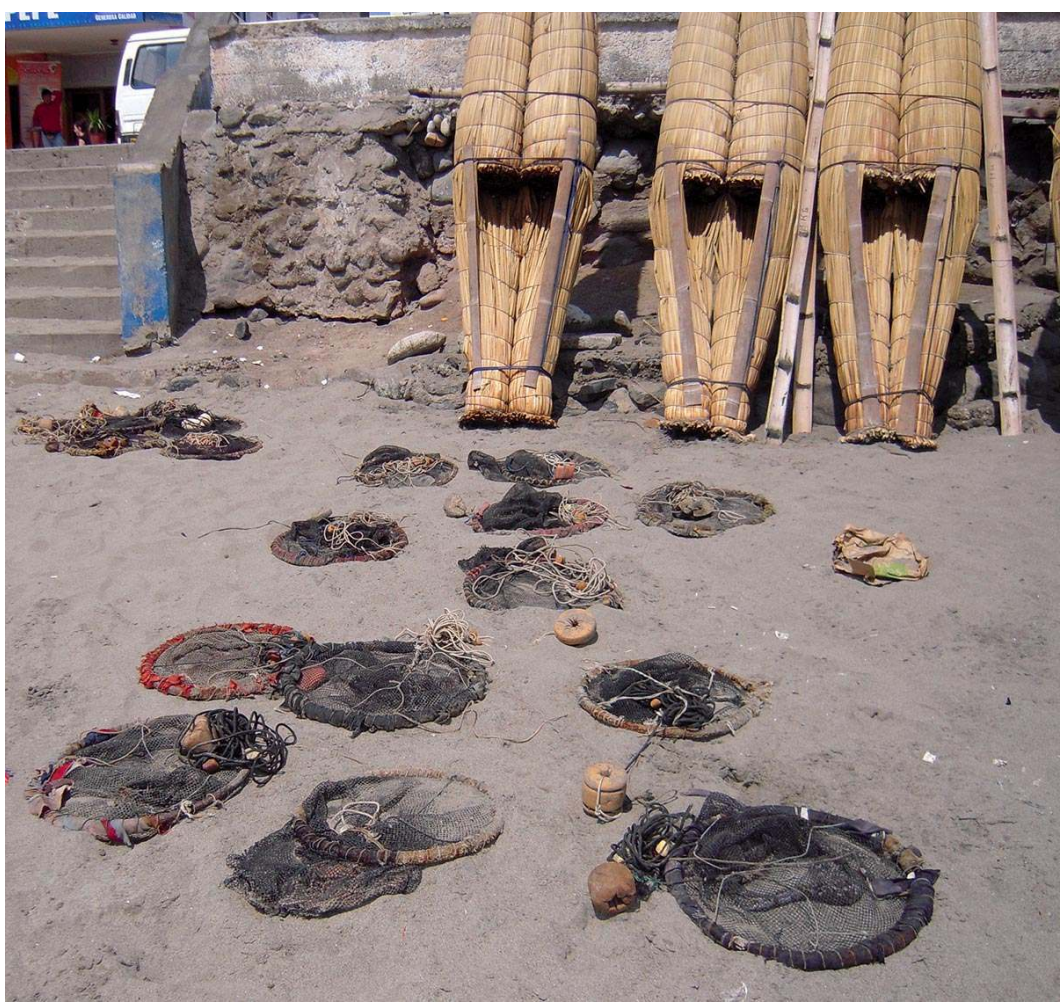

(c) M. Quiñe

55) Deux pièges-nasses à coquillages de Sicile 


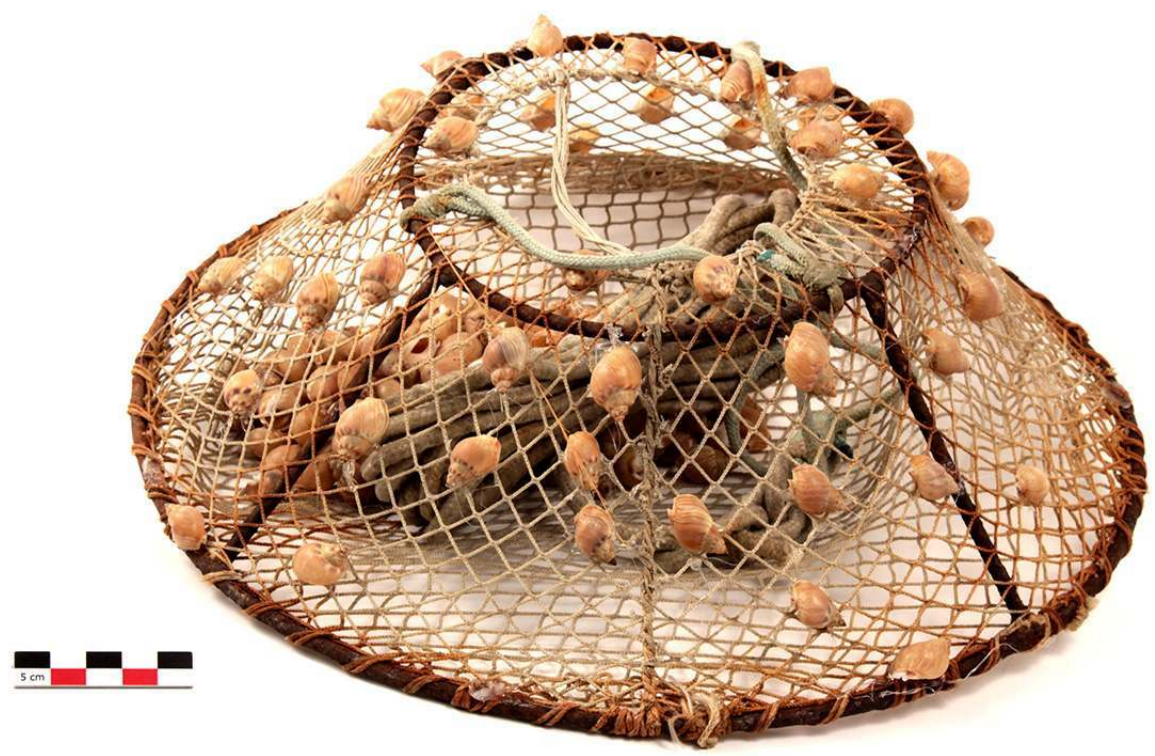

(c) P. Rameau

- Italie ; Catane, Sicile

- Nasse pour les coquillages Tritia mutabilis (ex-Nassarius); nasse per la pesca delle lumachine di mare. Cet engin est utilisé par les pêcheurs à la piccola pesca de Sicile. Ce petit coquillage, lumachine en italien, "nasses changeantes », en français, est un mollusque nécrophage qui s'appâte avec des poissons morts. L'engin de pêche est constitué d'un cadre en fil de fer et d'un corps en toile de filets. Il se pose au fond, et se remonte avec un filin. Nos deux engins ont été offerts par Agostino Calogero, ancien fonctionnaire de la capitainerie maritime de Catane. Le montage des coquillages a été réalisé par lui à titre démonstratif

- Octobre-novembre 2015 ; collectrice Nastassia Reyes

- Diamètre $32 \mathrm{~cm}$, hauteur $10 \mathrm{~cm}$

- ETB-IT-NR-2015-001 et 002

Matériel annexe : récipients pour mettre les prises

56) filet-sac à poisson 


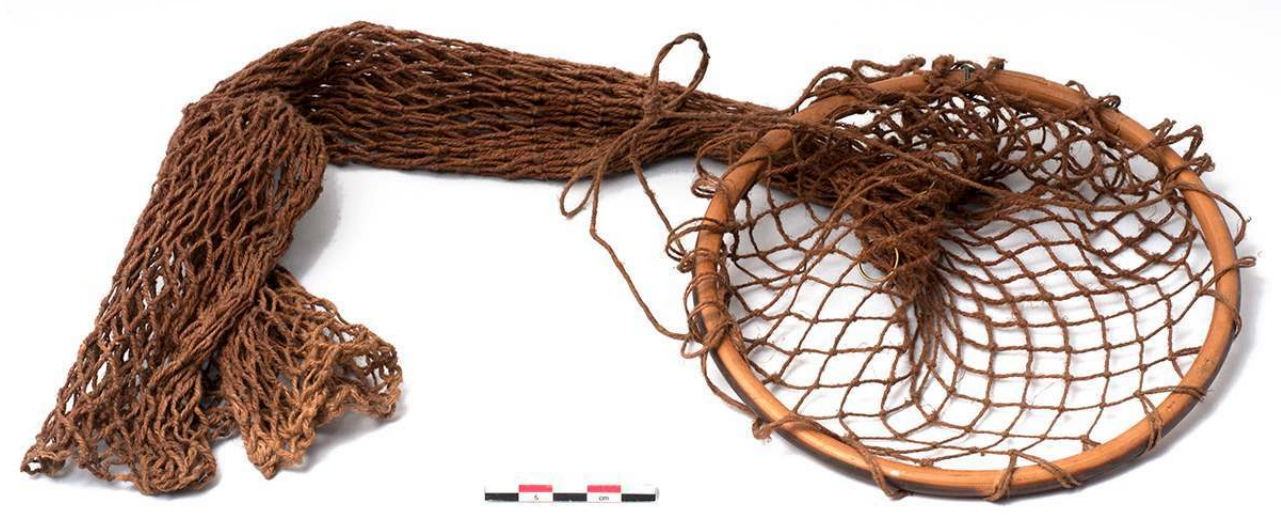

(C) J.C. DOMENECH

- France

- Filet en fibre végétale de lin, avec un anneau en rotin. Sac à poissons pour la pêche dans les rivières, pour mettre les prises

- Années 1970; collecteur Raymond Pujol

- Longueur $57 \mathrm{~cm}$, diamètre $16,5 \mathrm{~cm}$

- ETB-FR-RP-2011-004

\section{7) Paire de paniers du Vietnam}

Figure 76 : Paire de paniers vietnamiens ETB-VN-ALB-2009-003

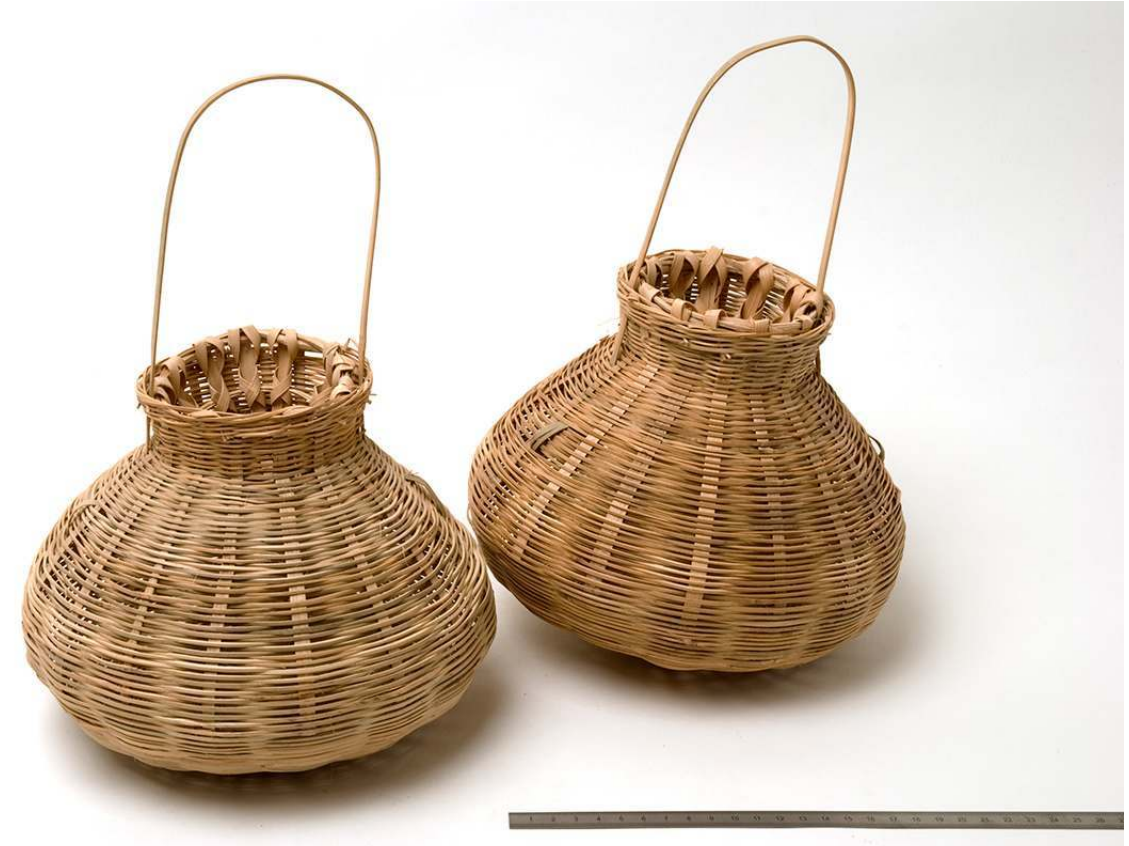

\section{(C) J.-C. DOMENECH}

171 • Vietnam ; Mangrove de Can-Gio, Hô Chi Minh Ville

- Paire de paniers à petits poissons et crevettes. Bambou. Paniers à bouchon en entonnoir, 
portés à la hanche par les femmes et les hommes; destinés à contenir les prises lors des pêches à la main ou à l'épuisette dans les rizières

• 6/2008; collectrice Ariadna L. Burgos

- Hauteur du panier $18 \mathrm{~cm}$, diamètre $22 \mathrm{~cm}$, anse $11 \mathrm{~cm}$

- ETB-VN-ALB-2009-003a et b

58) Panier à petits poissons du Laos

Figure 77 : Panier à poisson du Laos ETB-LA-FA-2017-015

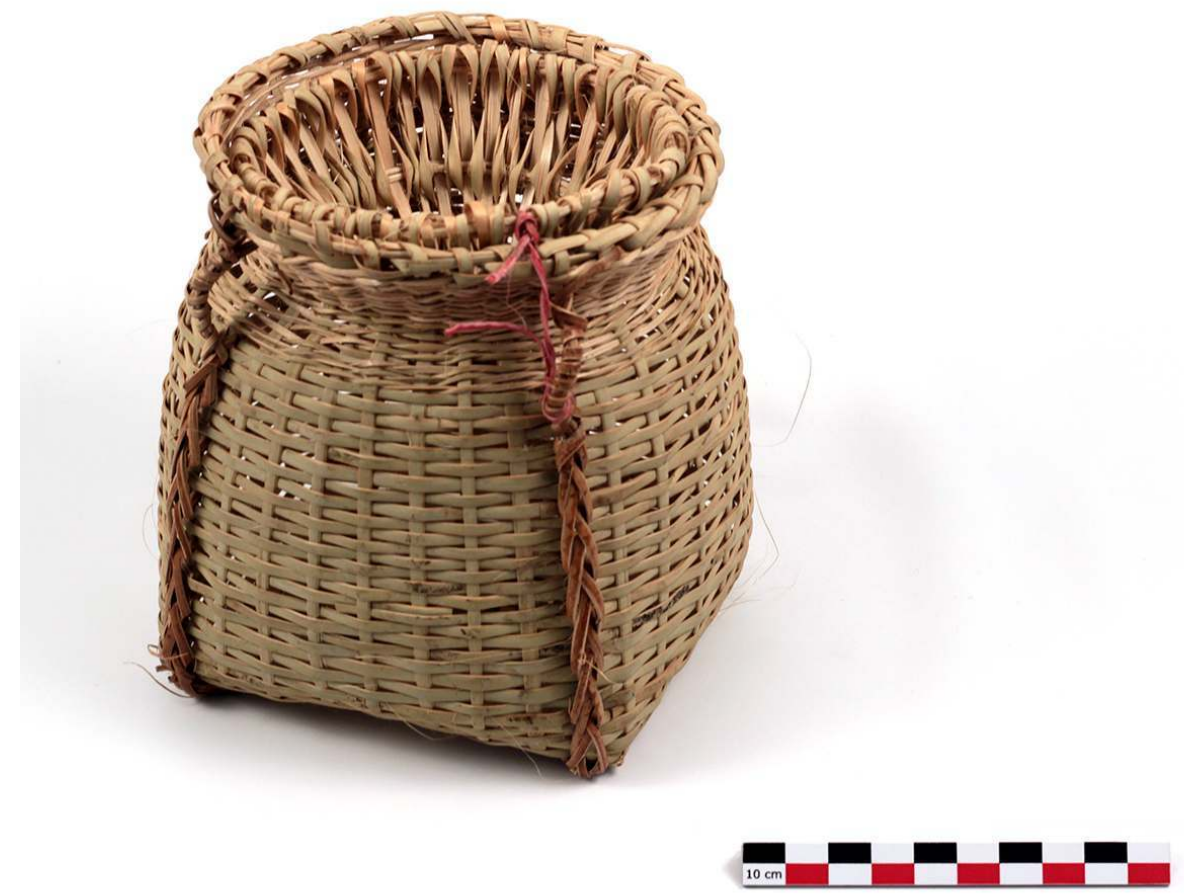

\section{(c) P. Rameau}

173 • Ethnie Lao. Laos ; village Vieng Tong, Province de Huapan

- Bambou. Pendu à l'épaule ou porté à la ceinture, tant par les hommes, les enfants que par les femmes, au cours de leur travail dans les rizières et les petits cours d'eau, pour y placer les prises. Le couvercle en entonnoir les empêche de ressortir - Décembre 2017 ; collectrice Françoise Aubaile

- 13 x $12 \mathrm{~cm}$, hauteur $14,5 \mathrm{~cm}$

- ETB-LA-FA-2017-015 
Figure 78 : Utilisation du panier d'aisselle au cours d'une pêche collective au Laos (d'après Claridge et al. 1997 : fig. 8 p. 37)

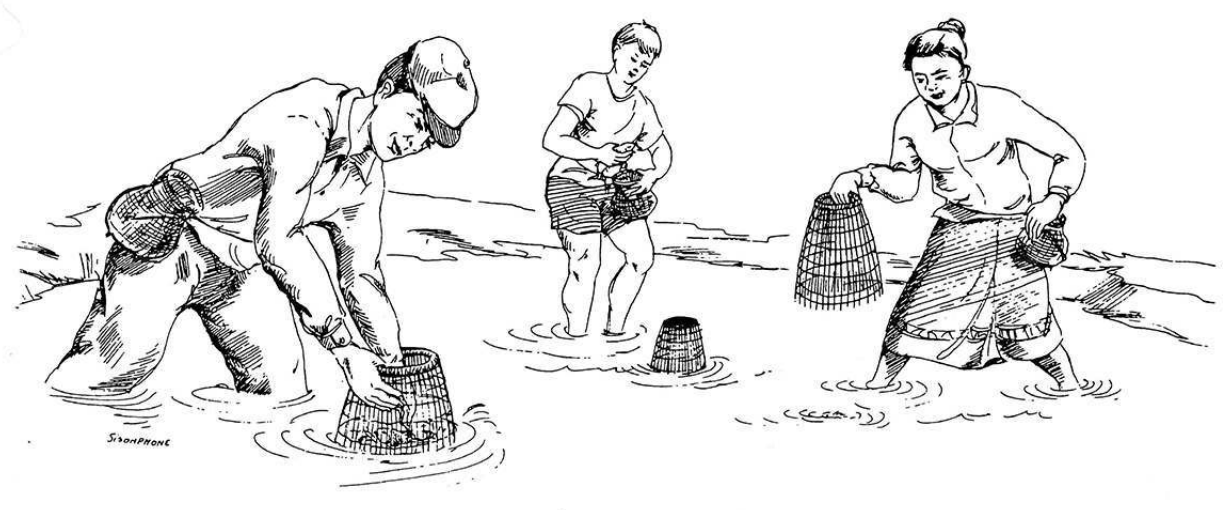

(c) S. Hansai

Engin passif, agissant par emprisonnement

Maillage

59) Modèle de filet du Pérou

Figure 79 : ETB-PE-MQ-2009-027

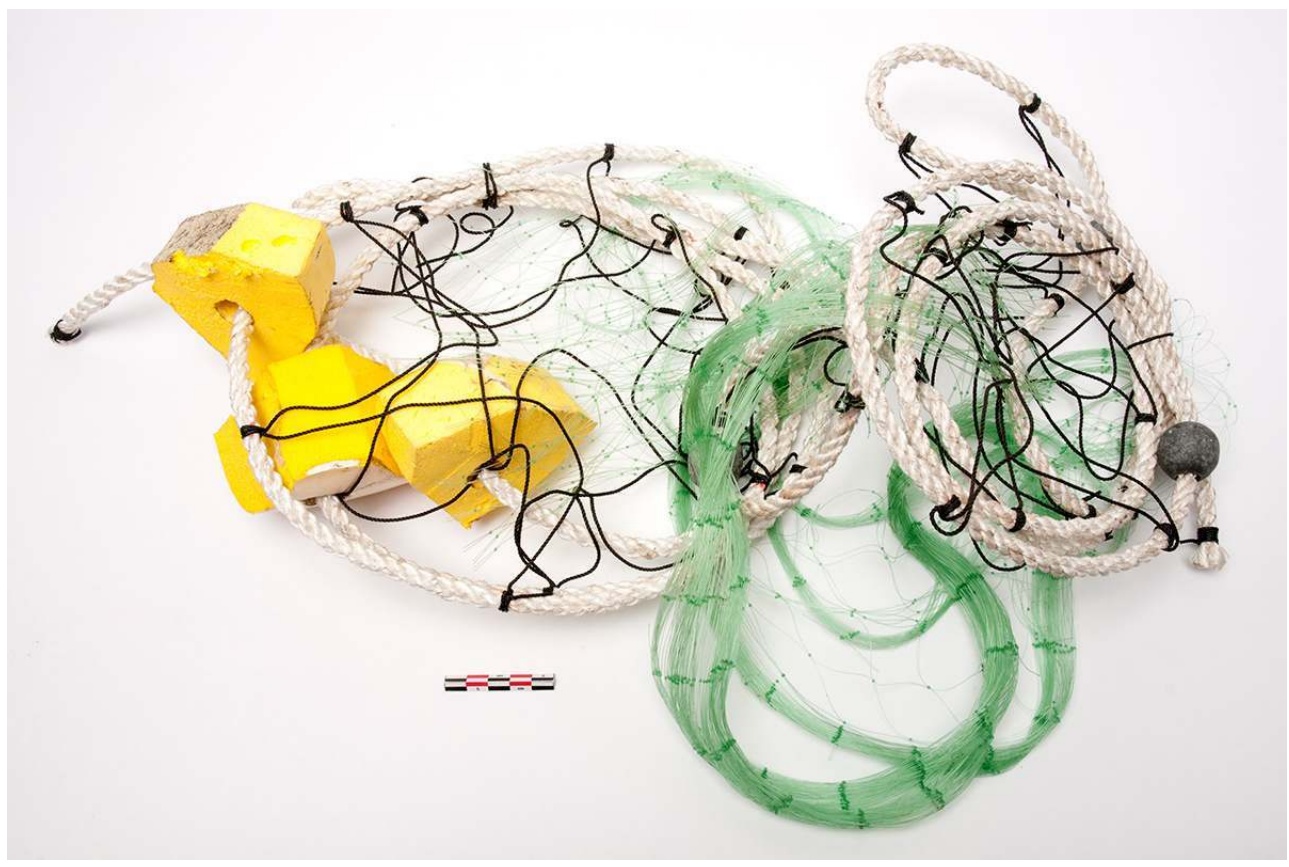

(c) J.C. Domenech

175 • Pérou, Salaverry, département La Libertad ; province Trujillo

- Red de pescar de un solo paño. Échantillon en réduction de filet de pêche à une seule nappe. Il est constitué de fils de nylon, corde synthétique et flotteurs 
-22/08/2009 ; collectrice Marina Quiñe

- ETB-PE-MQ-2009-027

Figure 80 : Ravaudage des filets devant les CABALLITOS, sur la grève

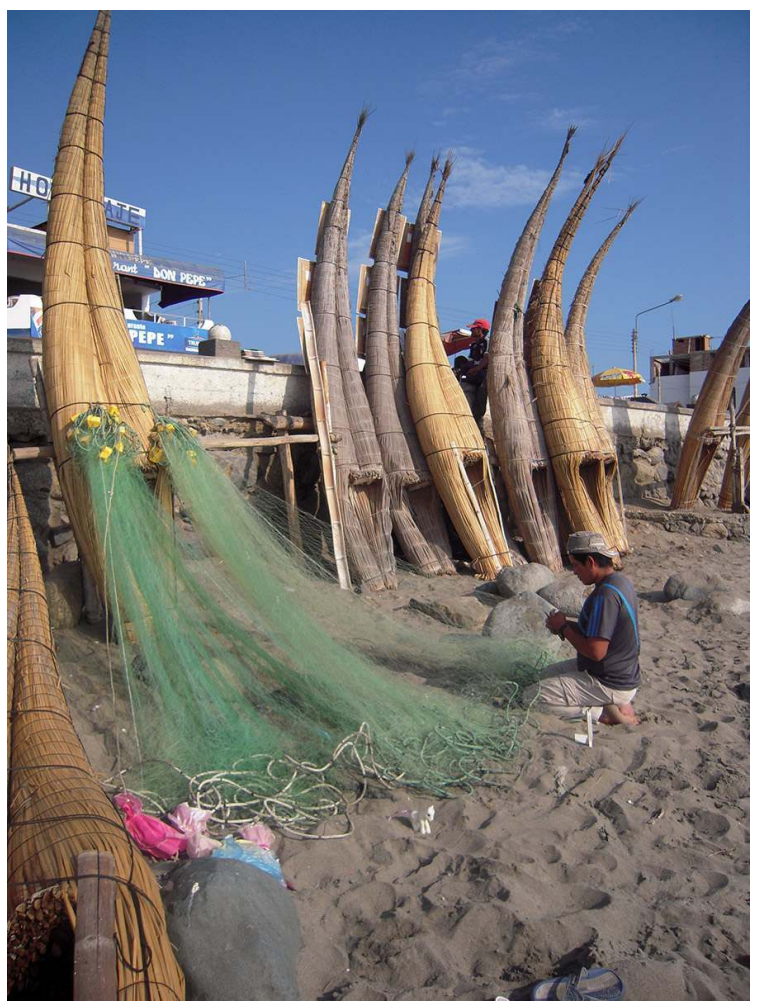

(c) M. Quiñe

60) Filet de pêche muni de flotteurs, Brésil

Figure 81 : ETB-BR-MC-2013-001

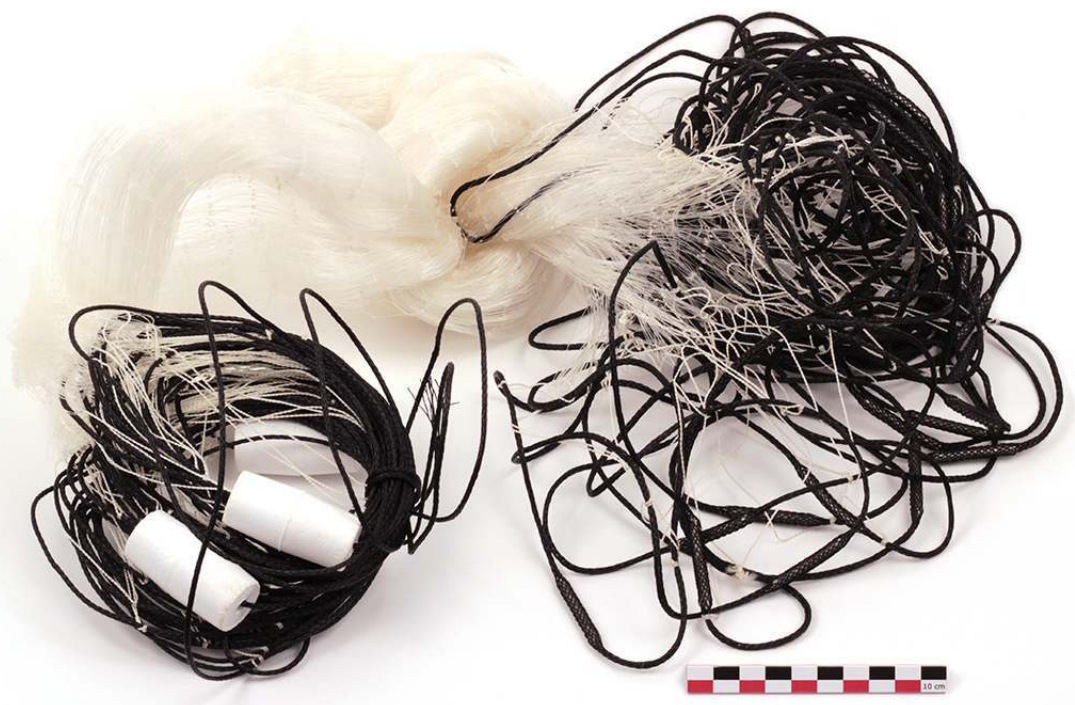


177 - Caboclos ; Brésil. Parintins, Amazonas. Achat dans une boutique spécialisée «A casa do pescador"

- Filet de pêche muni de flotteurs, pour les eaux douces. Pêche en eau douce sur les rivières amazoniennes, depuis des barques à moteur (rabetas) ou à rames

-16/04/2013 ; collectrice Mélanie Congretel

- Hauteur de la nappe $168 \mathrm{~cm}$

- ETB-BR-MC-2013-001

61) Filet à omble chevalier (Groenland)

Figure 82 : Filet à omble chevalier MNHN-E-2016.6.80

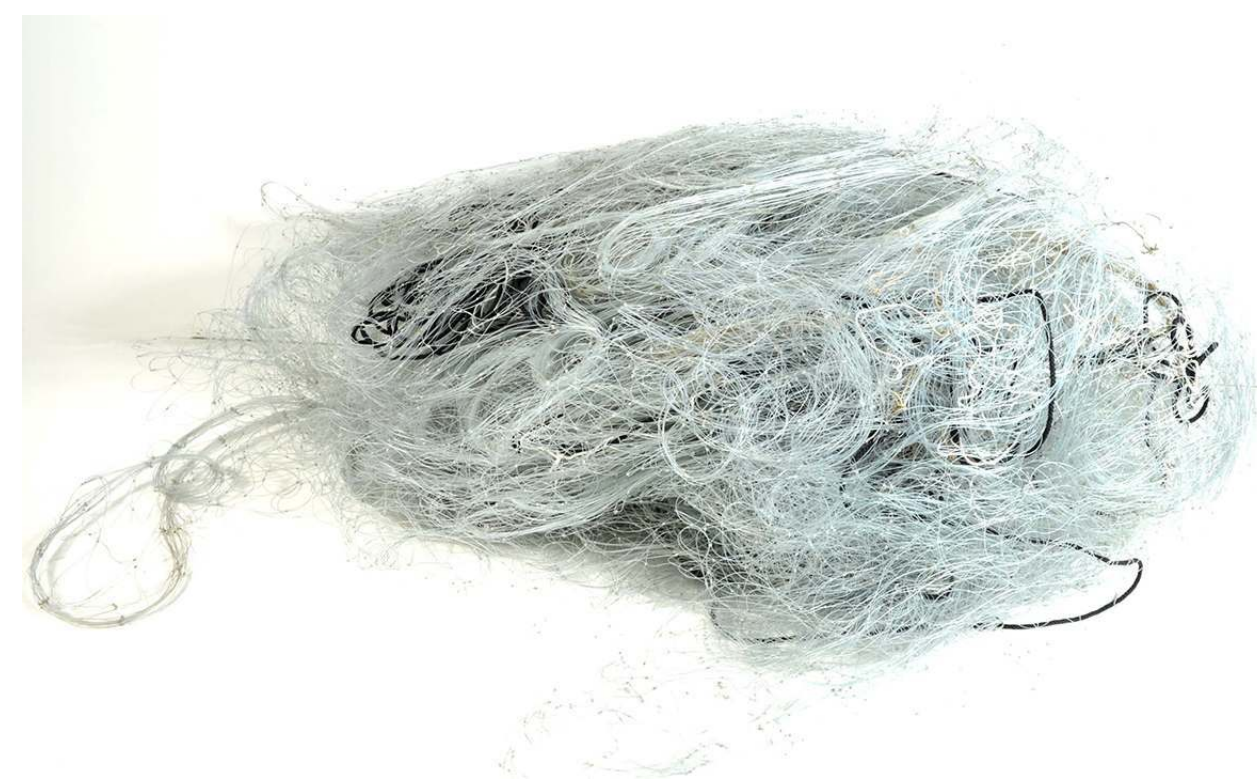

(c) D. Brabant

179 - Inuit ammasalimiut. Groenland, Ammassalik, Tasiilaq

- La pêche au filet des ombles chevalier kaporniangak (Salvelinus alpinus, Salmonidés) se pratique lors de leurs migrations des lacs vers la mer, en période d'eaux libres, à la fin de l'été. Les filets sont tendus à l'embouchure des torrents. (Robbe 1994 : 136-138)

- 2004 ; collecteurs Bernadette et Pierre Robbe

- MNHN-E-2016.6.80 
Figure 83 : En mer, le filet à omble peut aussi capturer de la morue ; c'est le cas ici avec Robartsi Umerineq dans la baie d'Aarngajak (1997)

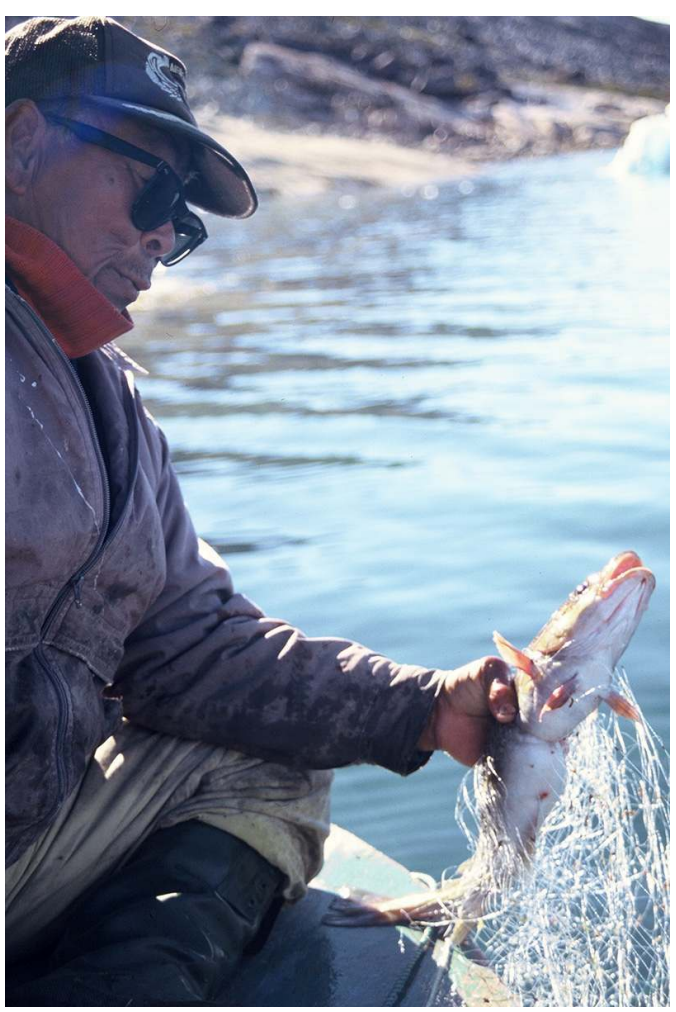

(c) P. Robbe

\section{2) Filet à phoque (Groenland)}

Figure 84 : filet à phoque MNHN-E-2016.6.81

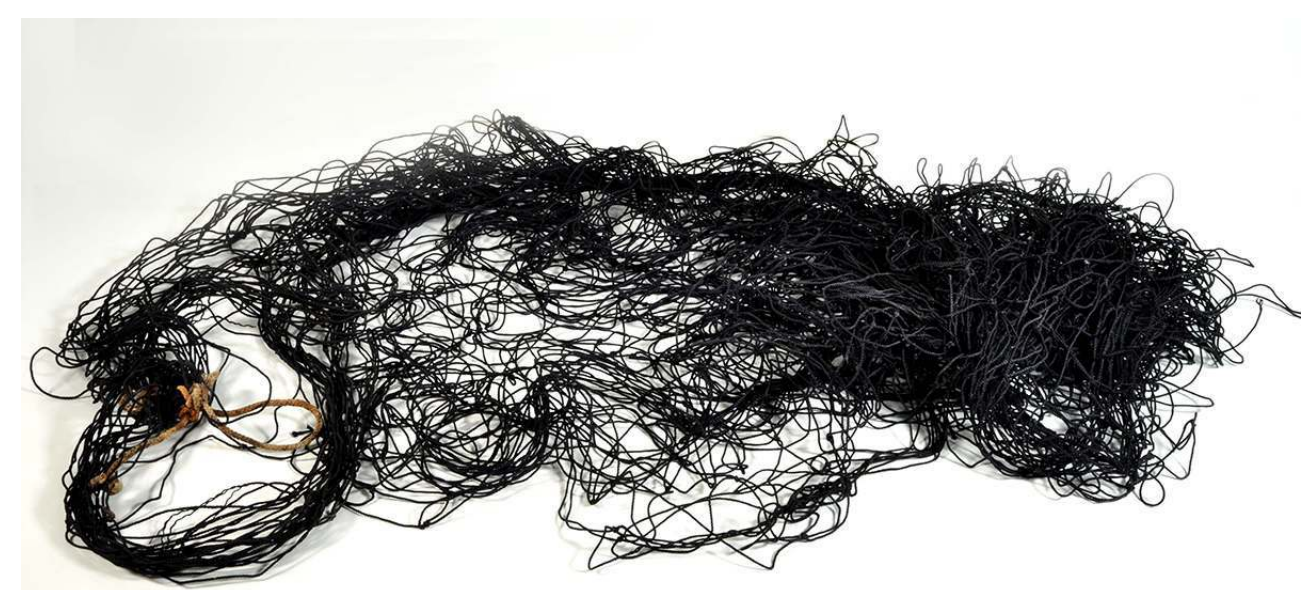

(c) D. Brabant

181 - Inuit ammasalimiut. Groenland, Ammassalik (côte est)

- Qatsulit. Fil de nylon noir, industriel. Capture des phoques au filet : sous la glace de la banquise, pendant l'hiver :

« les filets sont disposés le long de la côte, perpendiculairement à celle-ci [...]. La pose se 
fait par trois trous percés dans la glace. La distance entre les extrêmes correspond à la longueur du filet (10 à 12 m.) [...] Il est solidement accroché sur des blocs de glace ou sur des piquets, puis il est crocheté par le trou central pour poser une suspente intermédiaire qimiaq. Lesté à sa base par une dizaine de plomb, kippisaat, le filet pend sur environ trois mètres. Ses mailles font environ $15 \times 15 \mathrm{~cm}$. [...] le filet doit être relevé au moins une fois tous les deux jours [...]» (Robbe 1994 : 247)

- 2004 ; collecteurs Bernadette et Pierre Robbe

- MNHN-E-2016.6.81

\section{Entortillage}

\section{3) Modèle de filet tramail, Pérou}

Figure 85 : Modèle de filet tramail, Pérou, ETB-PE-MQ-2009-028

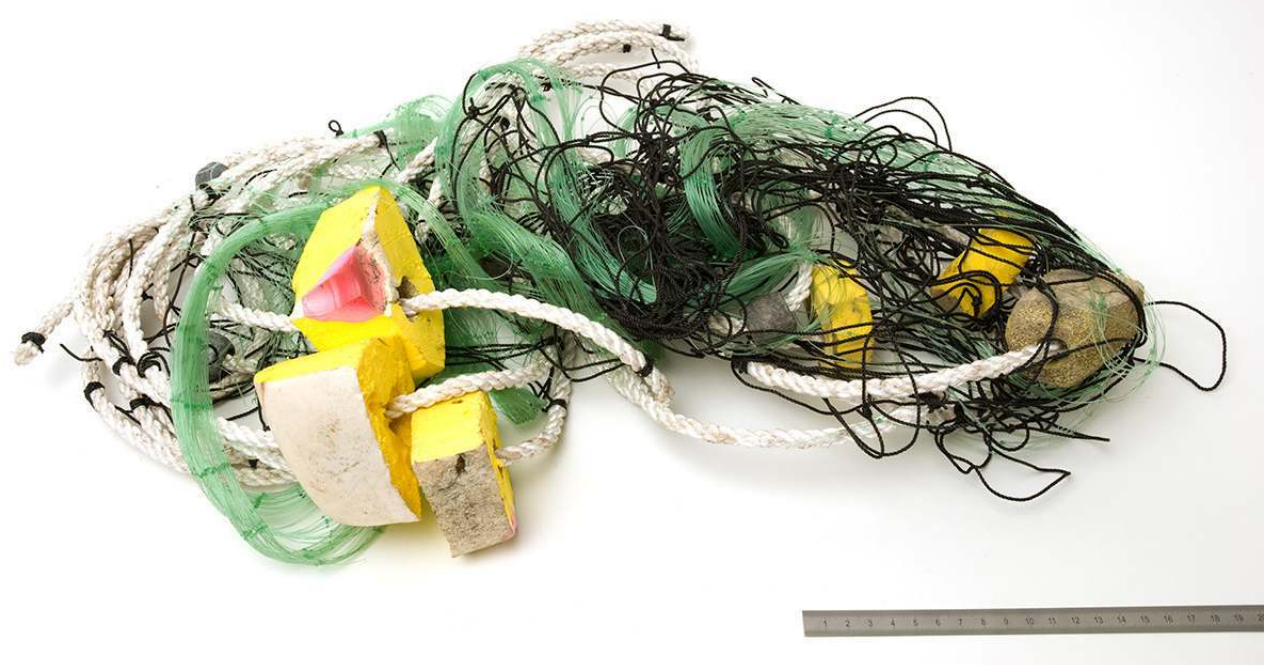

\section{(c) J.C. Domenech}

- Pérou, Salaverry, département La Libertad ; province Trujillo

- Red de transmallo. Modèle de filet tramail. Il est constitué de fils de nylon, corde synthétique et flotteurs. Un tramail (ou trémail) est constitué de trois nappes superposées : deux nappes à larges mailles, de part et d'autre d'une nappe moins tendue, à mailles serrées, de telle sorte que le poisson se trouve emprisonné dans une poche lorsqu'il passe au travers d'une nappe externe (Figure 78)

-22/08/2009 ; collectrice Marina Quiñe

- ETB-PE-MQ-2009-028 
Figure 86 : Comparaison entre un filet à nappe simple (à gauche) et un tramail (à droite)
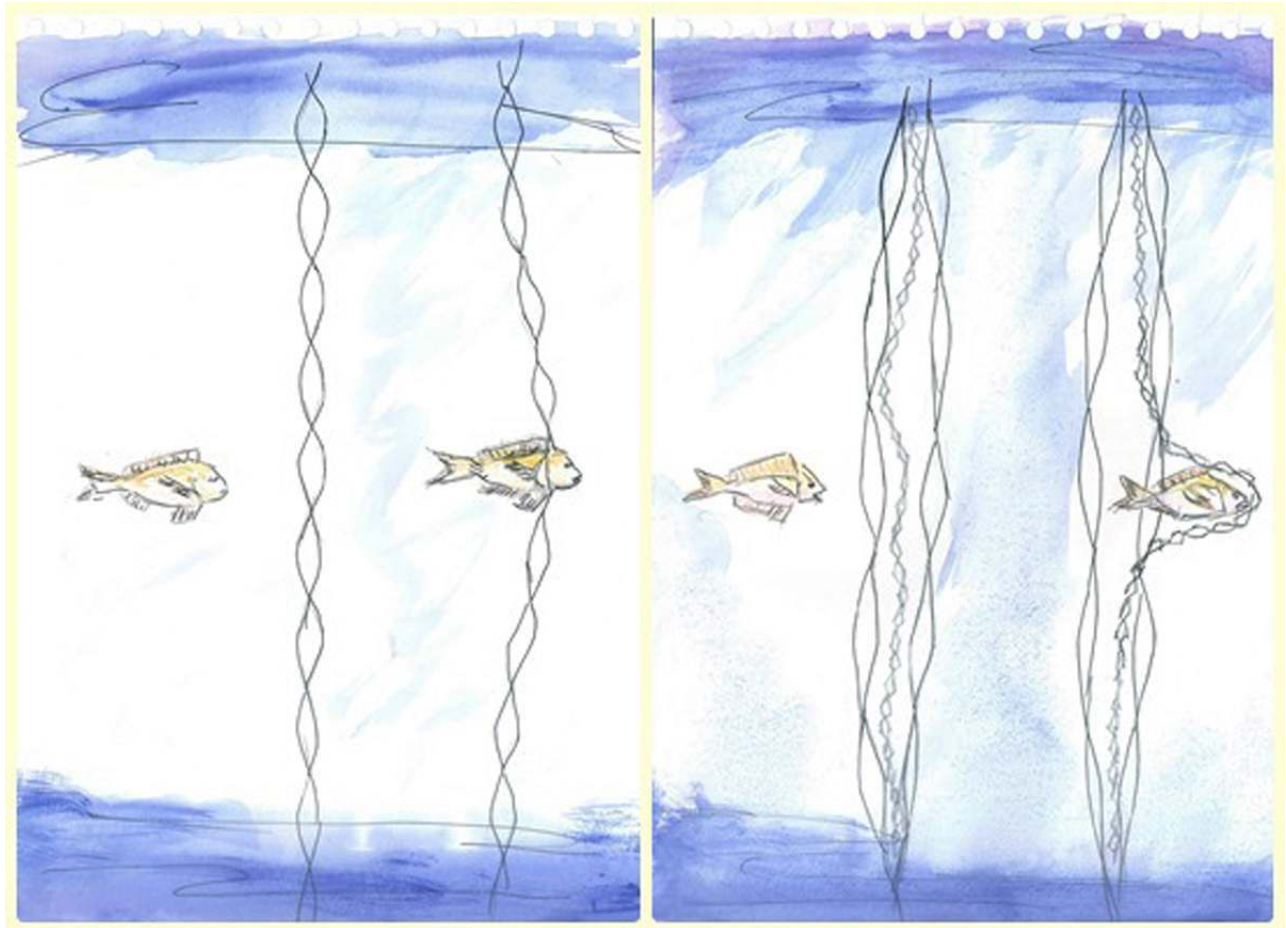

(C) N. Reyes

- Flotteurs pour les filets

64) Flotteurs de filets en verre (France)

Figure 87 : Paire de flotteurs en verre ETB-FR-SB-2018-004 (a et b)

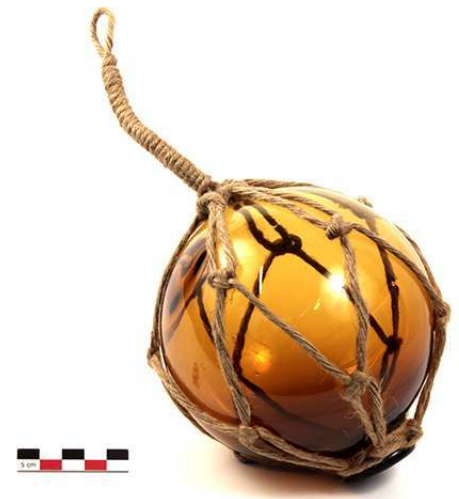

A

(C) P. RAMEAU

- France ; Arcachon, Gironde

- Flotteurs en verre pour filet. De nos jours ces objets classiques servent à la décoration des demeures ou des salles de restaurant

- Février 2012 ; collecteur S. Bahuchet (don de la promotion EDTS 2012)

- Diamètre $14 \mathrm{~cm}$, longueur $30 \mathrm{~cm}$

- ETB-FR-SB-2018-004 (a et b) 


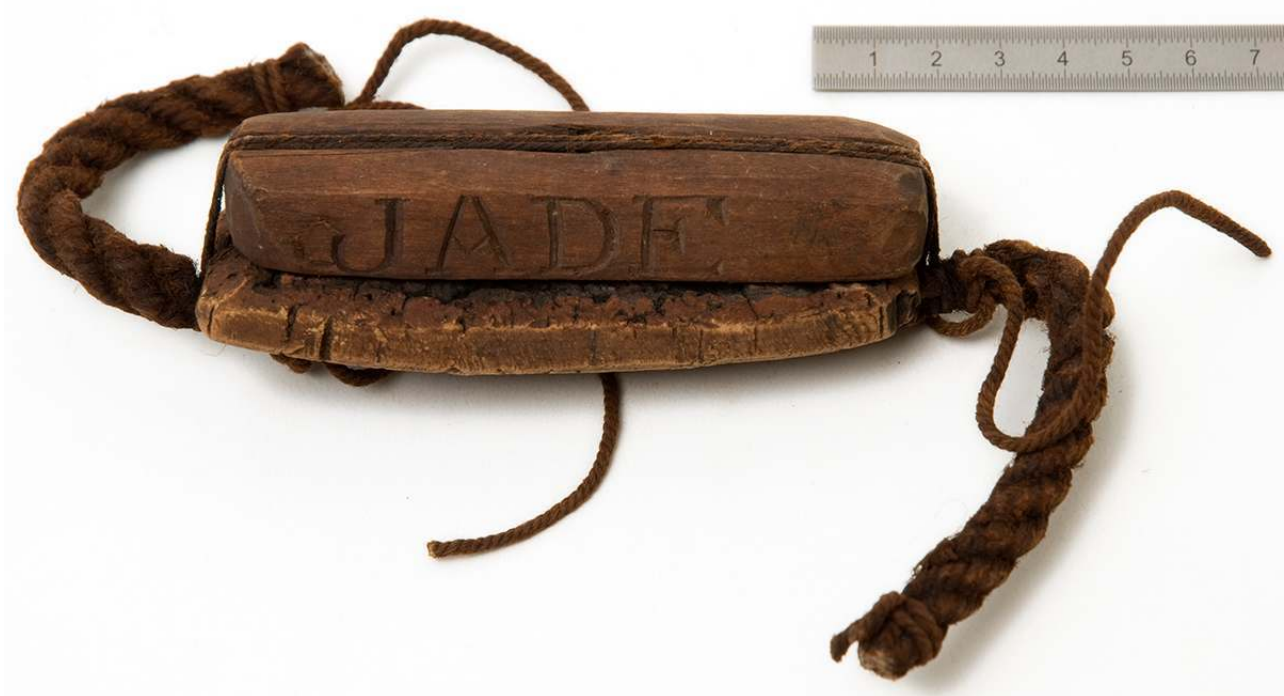

\section{(C) J.-C. Domenech}

188 • France ; Douarnenez, Finistère ; achat en brocante

- Flotteur-marque de filet de pêche ; Flotteur de filet en liège et bois, portant gravé le nom du pêcheur, FRANÇOIS sur une face, JADE sur l'autre

- Août 2008 ; Serge Bahuchet

- Longueur $11 \mathrm{~cm}$, largeur $4 \mathrm{~cm}$, épaisseur $3,5 \mathrm{~cm}$

- ETB-FR-SB-2009-004

66) Flotteur pour filet de pêche (Libéria) 


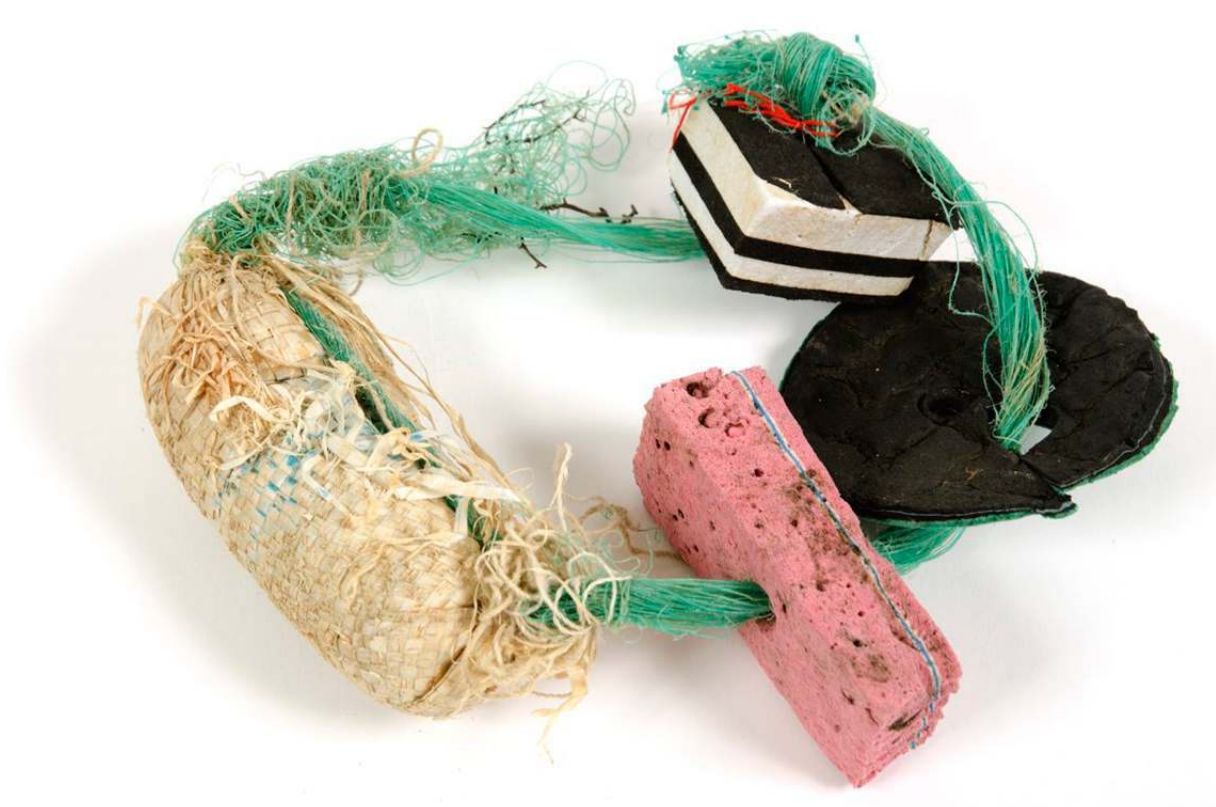

(C) D. Brabant

- Libéria

- Flotteur pour filet de pêche composé d'un lest et de trois flotteurs proprement dits

- 2012 ; collecteur Carlo de' Reguardati

- Ficelle : diam. $16 \mathrm{~cm}$ env. ; Lest : long. 10,5 cm env. ; diam. $5 \mathrm{~cm}$ env. ; Flotteur circulaire : diam. $9 \mathrm{~cm}$ env.; ép. $1,5 \mathrm{~cm}$; Premier flotteur: $8 \times 3,5 \times 2,5 \mathrm{~cm}$; Deuxième flotteur: $6 \times 4,5 \times 3 \mathrm{~cm}$

- MNHN-E-2012.6.14

\section{- Nécessaire de réparation}

67) Crochet pour ravauder les filets de pêche (Libéria)

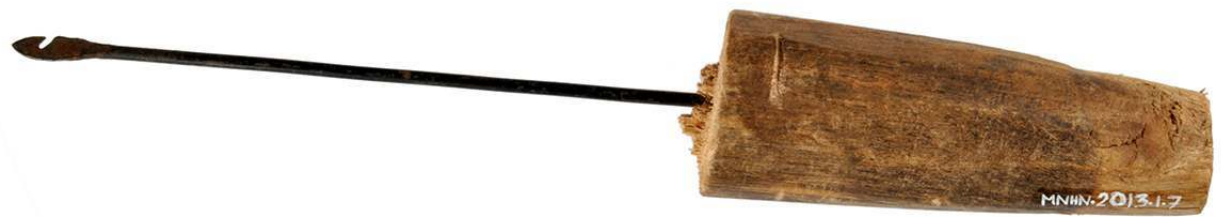

(C) D. Brabant

193 - Libéria, Suakoko, Comté de Bong

- Crochet servant à réparer les filets de pêche. Constitué du crochet proprement dit, en fer, se présentant en fine tige à l'extrémité aplatie et entaillée, et du manche cylindrique en bois. Pour les filets de pêche en rivière. Les pêcheurs sont d'origine étrangère 
(Ghanéens, Ivoiriens...). Fabriqué par Isaac Blamoh, forgeron

-22/10/2012 ; collecteur Carlo de' Reguardati

- Long totale : $15,6 \mathrm{~cm}$; Aiguille : $9 \mathrm{~cm}$, Manche $: 6,3 \mathrm{~cm}$

- MNHN-E-2013.1.7

\section{Nasses, avec un dispositif d'entrée}

\section{Figure 91 : Grand verveux pour filet fixe}

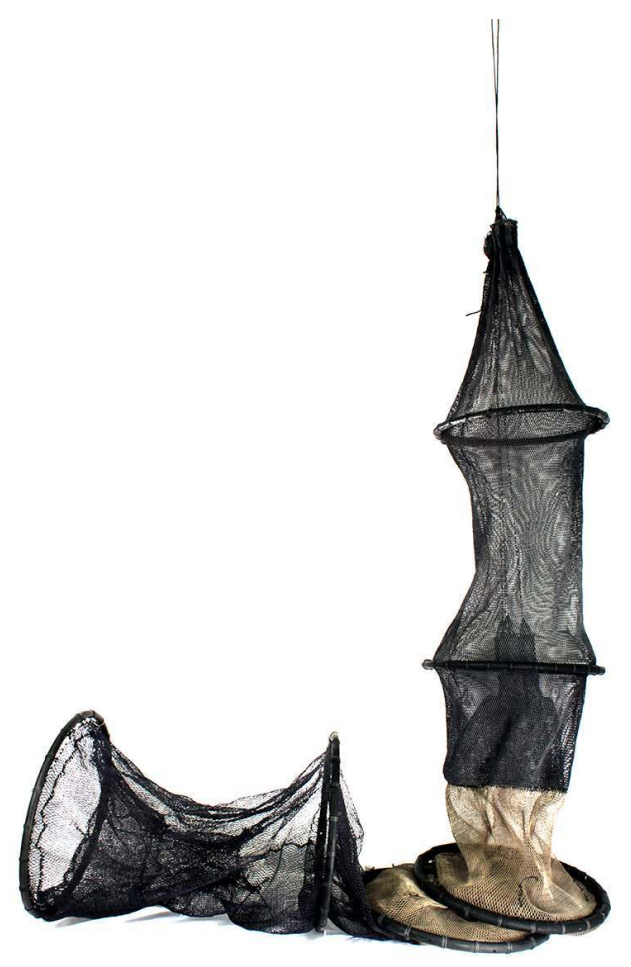

(C) P. Rameau

- France ; Palavas les flots, Hérault

- Les filets fixes font partis des engins de pêche les plus couramment employés en Occitanie. Ils sont destinés à être utilisés dans les lagunes pour capturer différentes espèces de poisson mais notamment, les anguilles (Anguilla anguilla). Ils sont constitués d'une paladière, long filet allant du fond à la surface, tenue par des piquets régulièrement espacés, formant une barrière verticale. Sa fonction est de conduire le poisson vers les tours, lieux où se concentrent les verveux, sorte de nasses en forme d'entonnoir soutenues par des cerceaux, dans lequel est piégé le poisson. Quand il y est entré, il ne peut pas rebrousser chemin. C'est là que les pêcheurs le récupèrent. Le début de la paladière est généralement disposé perpendiculairement au rivage, en bordure de celui-ci et en allant vers les plus bas-fonds. Cet engin a été offert par Bruno Bousquet, pêcheur aux petits métiers de Palavas-les-Flots

- 2013 ; collectrice Nastassia Reyes 
- Diamètre $42 \mathrm{~cm}$, longueur $300 \mathrm{~cm}$

- ETB-FR-NR-2018-001

Pour retirer les poissons de l'extrémité de chacun des verveux où ils aboutissent, les pêcheurs font émerger l'une après l'autre, chacune des extrémités, qu'ils dénouent à l'intérieur de leur embarcation. Le poisson reste vivant et les pêcheurs peuvent, s'ils le souhaitent, relâcher les captures qui ne les intéressent pas. La mise en place de ces engins est relativement longue à effectuer, aussi ces engins ne sont entièrement sortis de l'eau que lorsque les filets sont recouverts de salissures ce qui influe de manière négative sur l'efficacité de la pêche. Ils sont alors mis à sécher sur les étendoirs de manière à ce que ces salissures disparaissent. Les embarcations sont pourvues de petits moteurs mais le travail est encore exercé à la perche, ce qui en fait un métier de pêche très physique. Les pêcheurs considèrent que mis à part le matériau, ce métier aurait peu évolué.

Par ailleurs, les filets fixes peuvent être disposés de diverses manières. Chacune d'entre elles porte un nom particulier parmi lesquels il convient de distinguer : le pénêt, le doublis, la tête, le triangle, la maniguière, les brandines, les capéchades.

Figure 92 : A- schéma des filets fixes ; B- vue des filets dans l'étang ; C- filets séchant, à terre

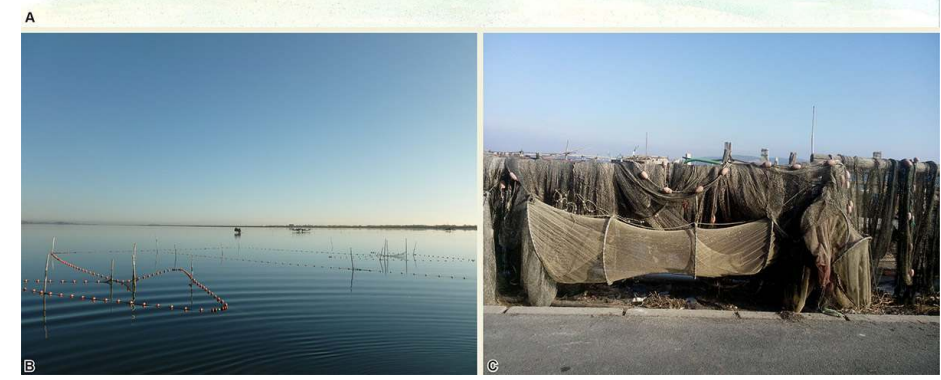

(C) N. Reyes

69) Nasse palikur à crevettes d'eau douce 
Figure 93 : Nasse palikur ETB-GF-SB-2014-42
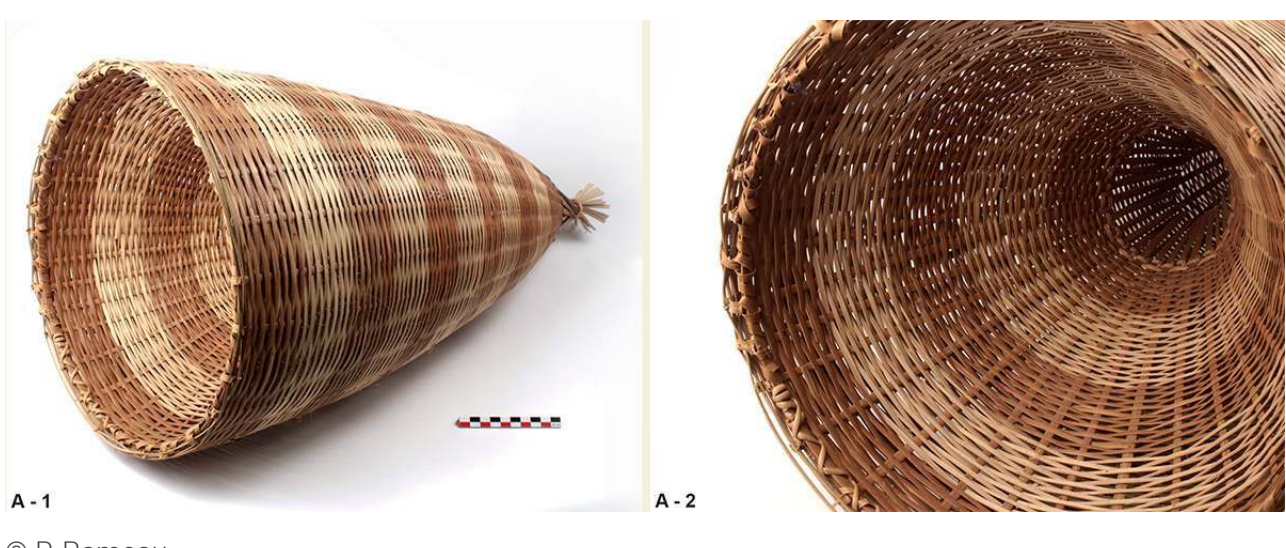

(C) P. Rameau

- Ethnie palikur. Guyane française, village Espérance 3, St Georges

- Takesati, nasse à crevettes d'eau douce; nasse conique en arouman Ischnosiphon sp., faite par Georgina Batista Norino; donnée à Pauline Laval. Fabriquée et utilisée par les Amérindiens palikur, sa forme est inspirée de la nasse à crevettes goli des Créoles de Guyane. En effet, cette forme conique est très largement attestée dans toutes les communautés de Guyane, tant amérindiennes que créoles, bien qu'elle tende à être supplantée par les filets tramail (Davy 2007 : 179). Les Palikur du village de Saint-Georges utilisent le takesati pour capturer les crevettes d'eau douce (appelées takes). On place des appâts à l'intérieur, des morceaux de manioc par exemple. Le piège est déposé dans l'eau, il est surtout utilisé dans les rivières affluentes de l'Oyapock, telles la rivière Gabaret. Pour récupérer les crevettes, on détache le lien situé à l'extrémité pointue de la nasse, les brins se desserrent et les crevettes tombent - 8/2014; collecteurs Pauline Laval et Serge Bahuchet

- Longueur $75 \mathrm{~cm}$, diamètre $35 \mathrm{~cm}$

- ETB-GF-SB-2014-42 
Figure 94 : Préparation des brins d'arouman pour la vannerie d'une nasse

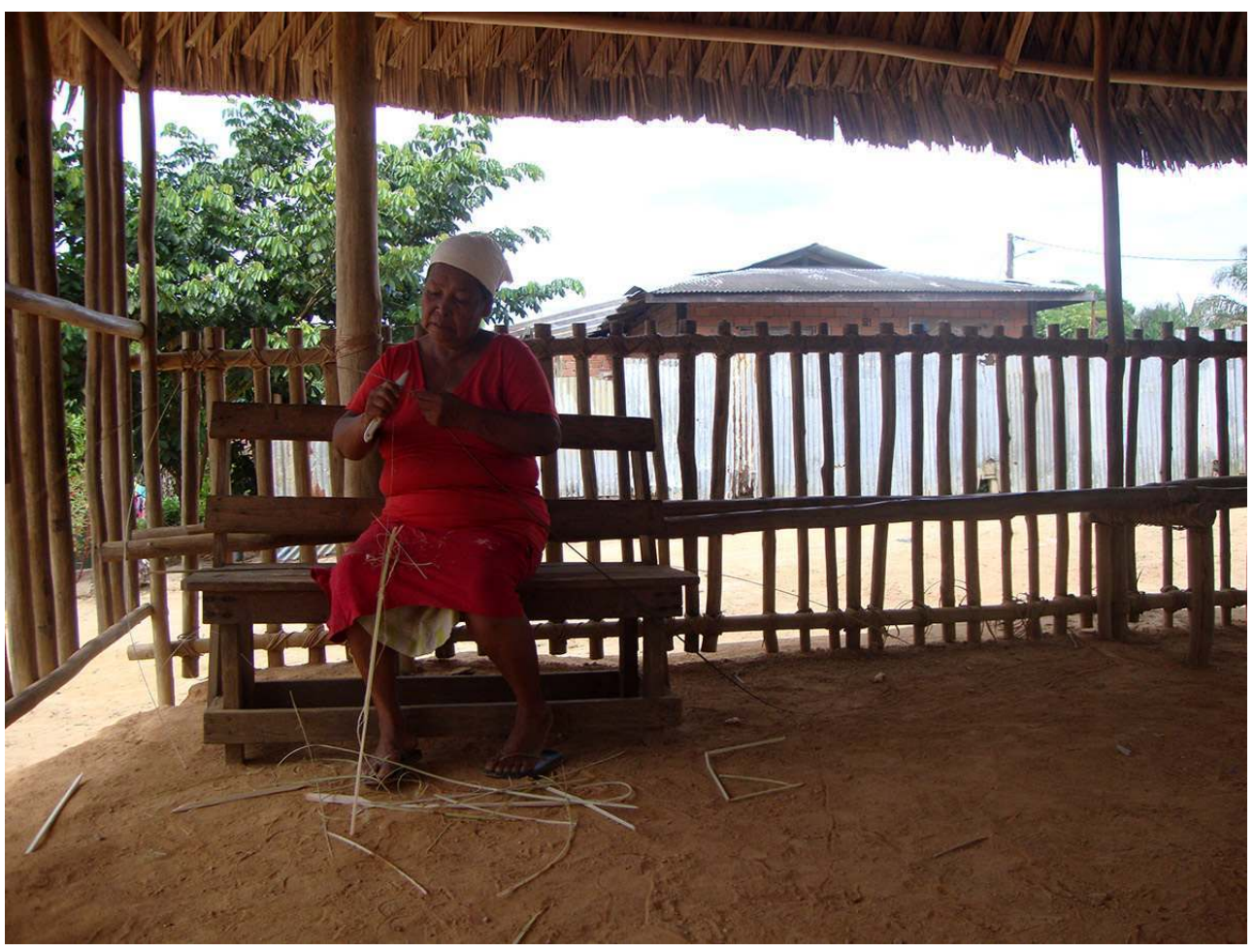

(c) P. Laval

70) Nasse à crevettes palikur

Figure 95 : Nasse à crevettes ETB-GF-SB-2014-15

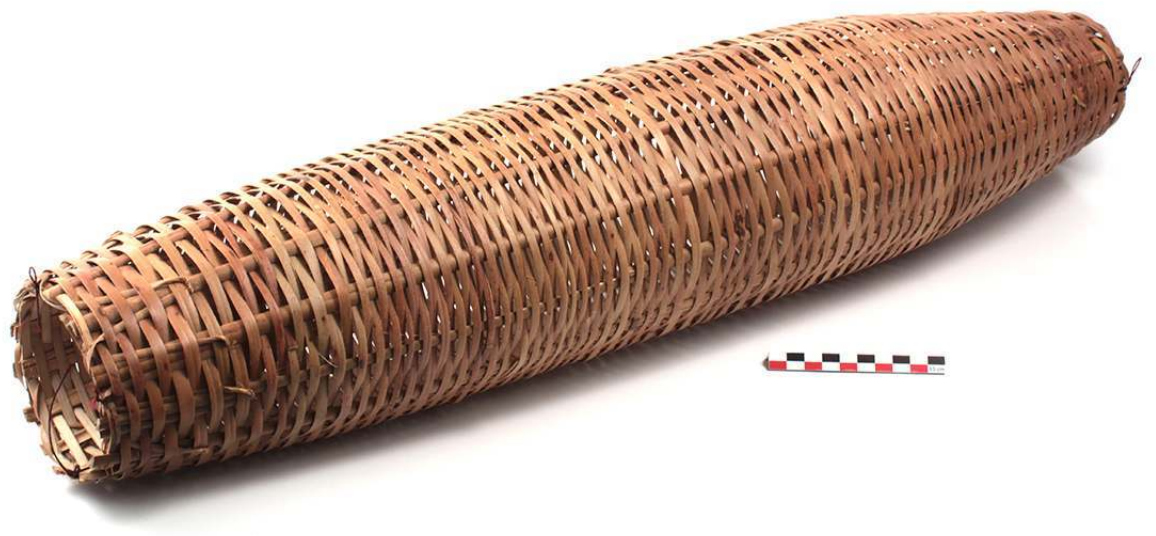

(c) P. Rameau

- Ethnie Palikur. Guyane française ; achat magasin association Gadepam, Cayenne

- Piège à crevettes en fibres d'arouman Ischnosiphon spp. ; fabriqué par Judith Voorburg. Deux entrées

-13/08/14 ; collecteur Serge Bahuchet 
204 - Longueur $73 \mathrm{~cm}$, diamètre $14,5 \mathrm{~cm}$

- ETB-GF-SB-2014-15

Figure 96 : Nasse (Libéria) MNHN-E-2012.6.13

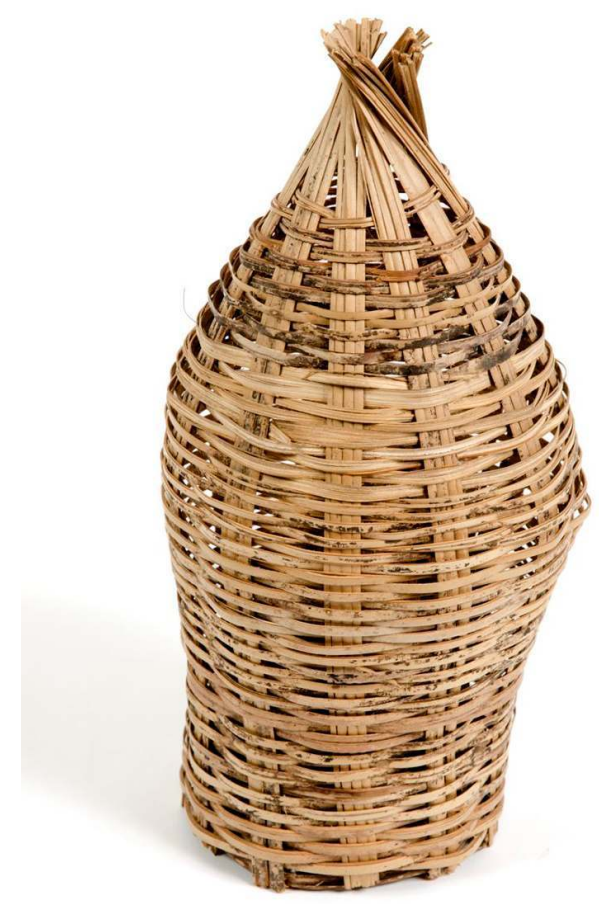

(c) J.C. Domenech

- Libéria

- Nasse à poissons de rivières tressée en fibres de rotin Laccosperma (Arécacées). Forme d'amphore, à ouverture (base) circulaire et sommet en pointe ; la partie interne est en forme d'entonnoir, permettant aux poissons d'entrer dans la nasse mais qui les empêche de sortir

- Date de collecte ; collecteur Carlo de' Reguardati

- Longueur $29 \mathrm{~cm}$, diamètre $16 \mathrm{~cm}$

- MNHN-E-2012.6.13

72) Nasse du Libéria 


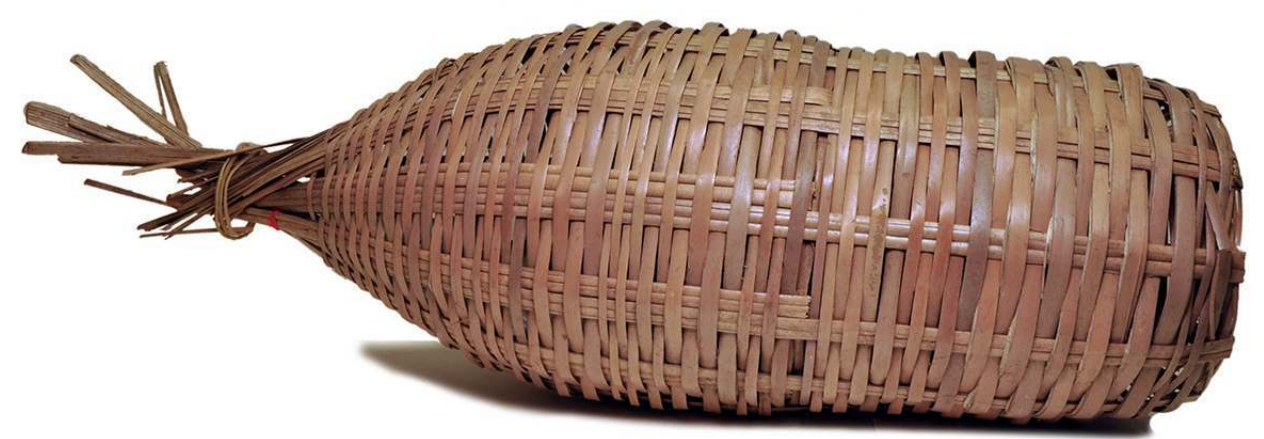

(C) D. Brabant

208 - Libéria, barrage électrique de Firestone

- Cet engin permet de pêcher des perches, des tilapias et de petits gardons. Pour lester la nasse, les pêcheurs mettent des cailloux à l'intérieur et des graines rouges de palmier à huile concassées comme appât. Utilisées par bouquets de 5 à 6 nasses, les nasses sont attachées à leur sommet par une longue liane de 10 à 15m (fichée dans la terre) et sont ensuite jetées dans les cours d'eau et les lagunes le soir. Elles sont récupérées le matin.

-11/2013 ; collecteur Carlo de' Reguardati

- Longueur $57 \mathrm{~cm}$, diamètre $18 \mathrm{~cm}$

- MNHN-E-2014.9.5

73) Nasse de Tunisie 


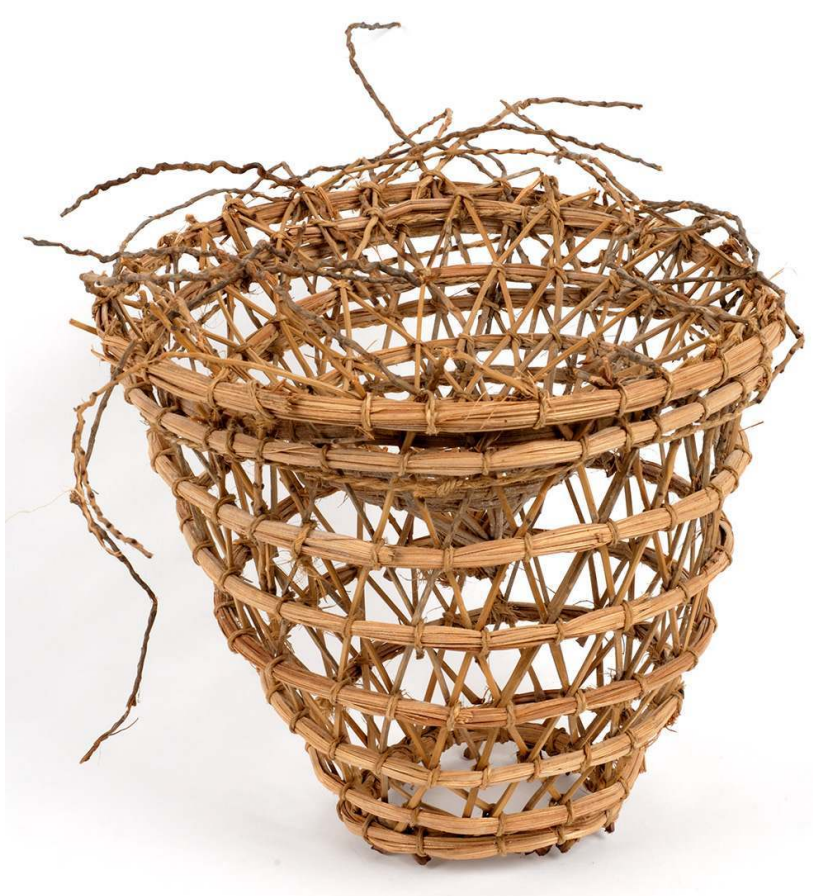

() J.C. Domenech

210 - Tunisie ; Ile de Djerba ; Marché des pêcheurs

- Nasse de Djerba dite drina, en palmier dattier (Phoenix dactylifera, Arécacées) (Romdhane 1998 : 67). Confectionnée à partir de l'inflorescence femelle (le régime de datte) : avec les épillets pour les brins, et les fibres de la hampe du régime, assemblées en faisceaux par de la ficelle, pour les anneaux. Cette nasse de petite taille est destinée aux calamars; des nasses de forme identique mais beaucoup plus volumineuses capturent les poissons. Le fond devra être fermé par un tressage de corde en forme de filet. L'entrée fermée sur cet exemplaire neuf, devra être détachée avant mise à l'eau

- 2003 ; collectrice Tatiana Fougal

- Hauteur $25 \mathrm{~cm}$, diamètre $28 \mathrm{~cm}$

- MNHN-E-2014.17.8

74) Nasse de Papouasie-Nouvelle Guinée 


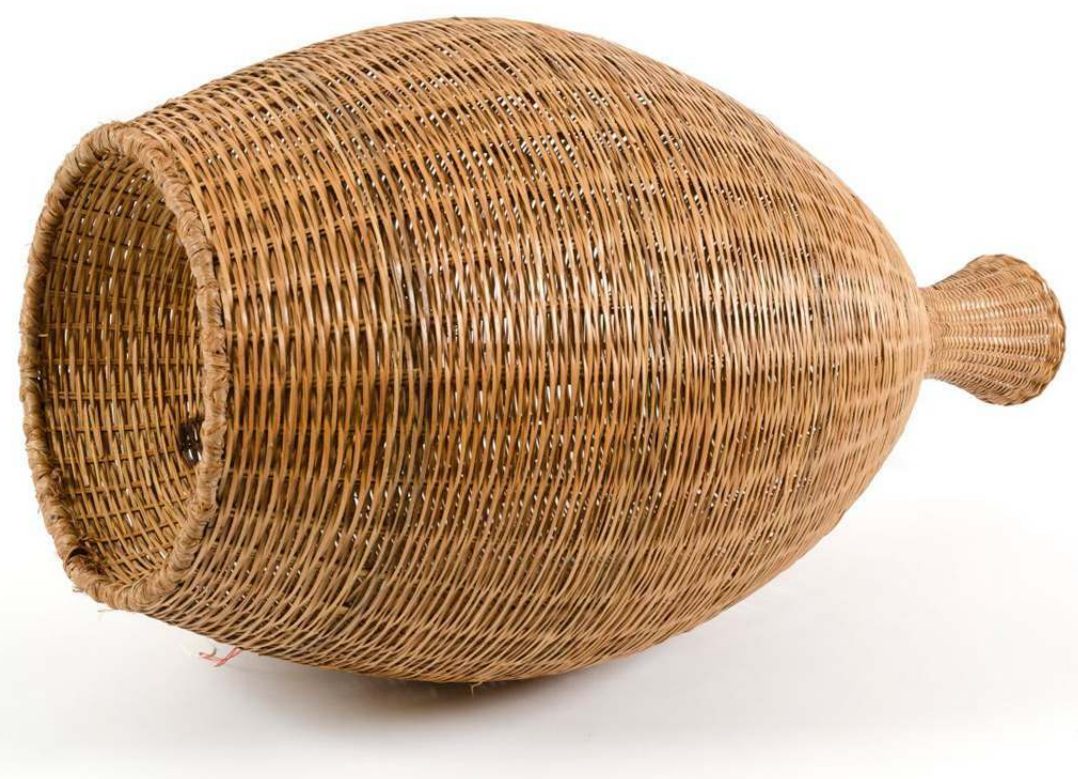

(C) J.C. Domenech

212 - Papouasie-Nouvelle Guinée ; Province de Morobe

- Acheté par le collecteur à des villageois, dans une rue de la ville de Lae. Nasse en vannerie constituée de nervures de folioles de palmier sagoutier (Metroxylon sp.) coupées en lattes verticales, sur lesquelles sont tressées de fines lanières ; renforcée à la base ; haut de la nasse resserré. Le fond est évasé pour permettre l'entrée des poissons, et resserré en entonnoir pour empêcher la sortie

- 1972 ; collecteur Christian Coiffier

- Longueur $80 \mathrm{~cm}$, diamètre ouverture base $29 \mathrm{~cm}$, diamètre ouverture dessus $12 \mathrm{~cm}$, diamètre au centre $40 \mathrm{~cm}$

- MNHN-E-2012.1.8

76) Nasse à crevettes cylindrique (Brésil) 


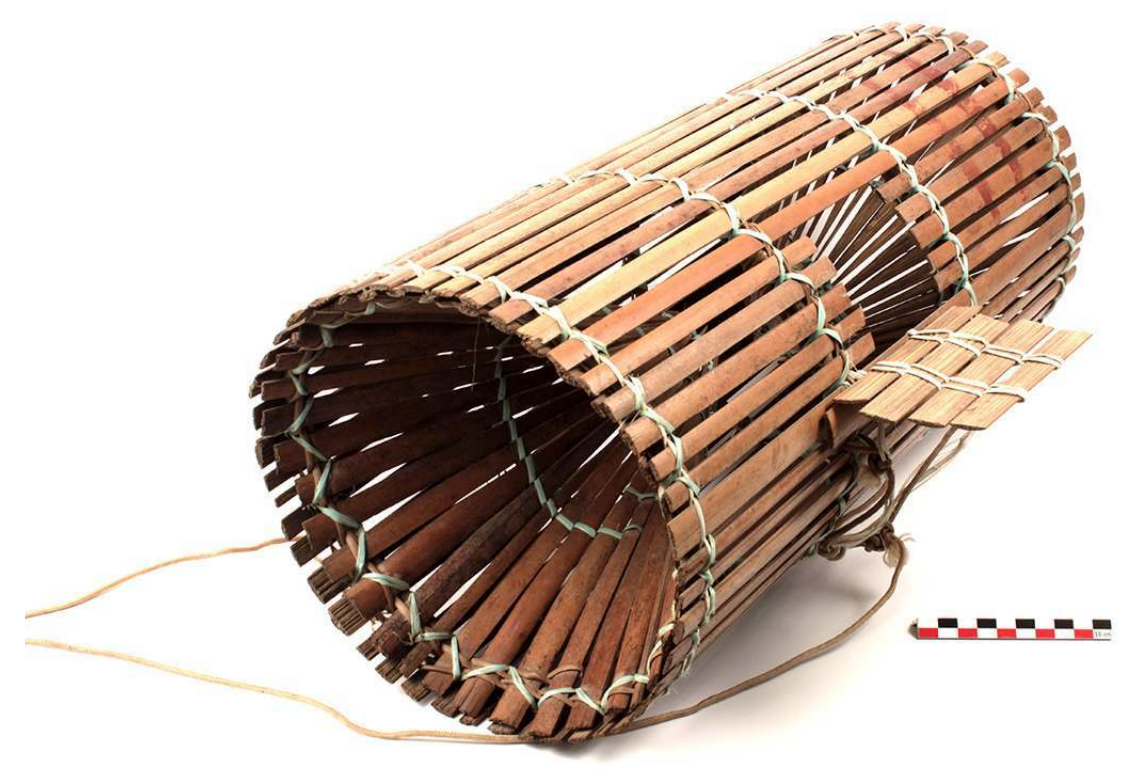

(c) P. Rameau

214 - Brésil ; provient d'Abaetetuba, Pará, acheté au marché de Belém (Pará, Brésil) par un pêcheur. Utilisé à Vila Vitoria (Amapá)

- Nasse à crevettes matapi en tiges de Raphia tardigera (Arécacées), tala de jupati, tandis que les liens, traditionnellement en liane, ont été découpés dans des sacs en plastique. Les deux extrémités sont ouvertes par un entonnoir. Un appât de farine de babaçu, enserré dans un sac plastique percé de petits trous, est déposé dans le piège (fig. 93). La trappe est maintenue fermée par un lien. La nasse est plongée dans le fleuve, tout en étant maintenue au moyen d'une ligne à un piquet planté sur la berge ou sur un ponton. Le pêcheur relève la nasse après quatre à cinq heures. Les crevettes sont évacuées par la trappe. (Laval $2016: 267$ )

-15/09/2013 ; collectrice Pauline Laval

- Longueur $47 \mathrm{~cm}$, diamètre $22 \mathrm{~cm}$

- ETB-BR-PL-2014-001 
Figure 101 : Préparation de l'appât en farine de BABAçU (Attalea speciosa, Arécacées)

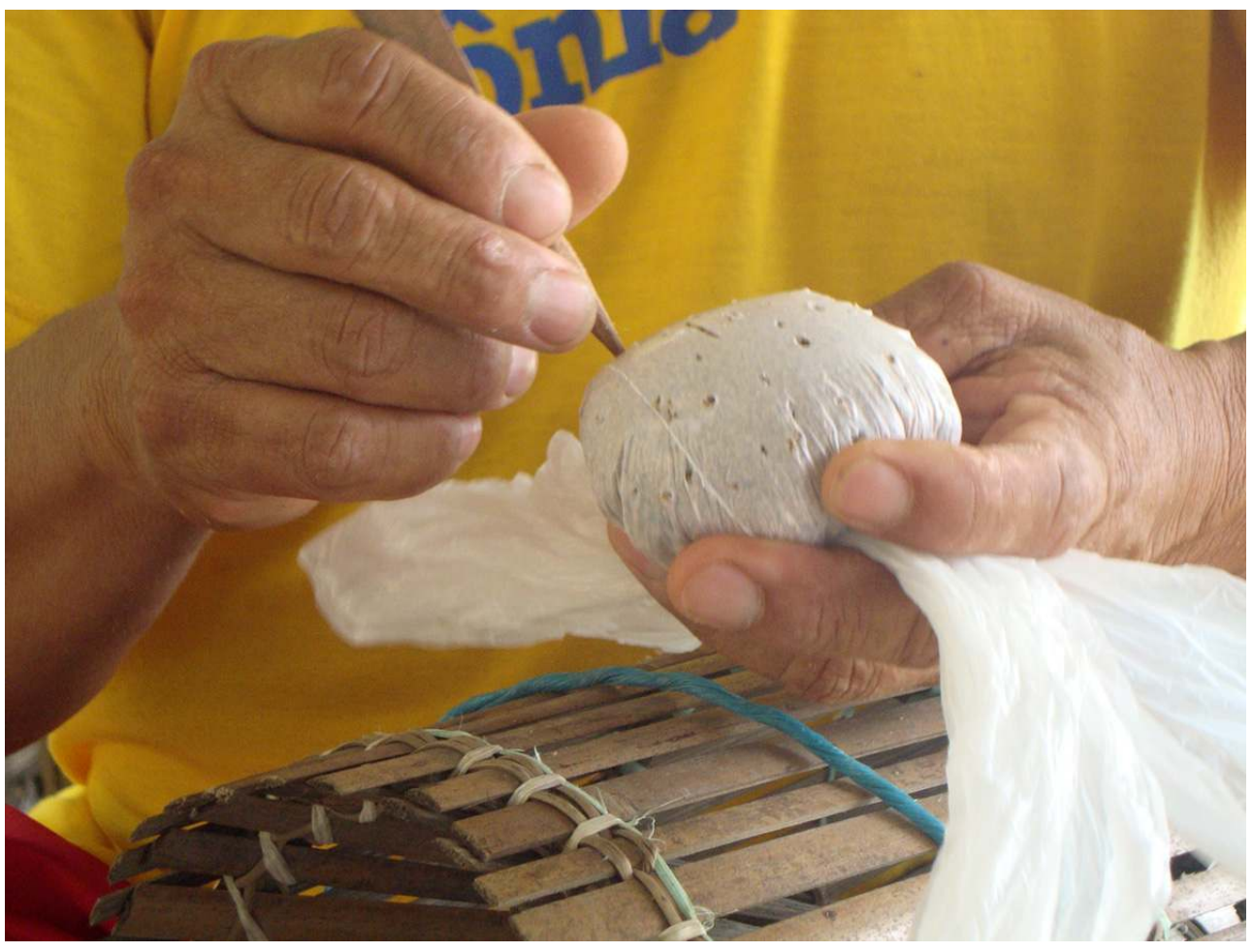

(C) P. Laval

Figure 102 : Les nasses dans l'eau

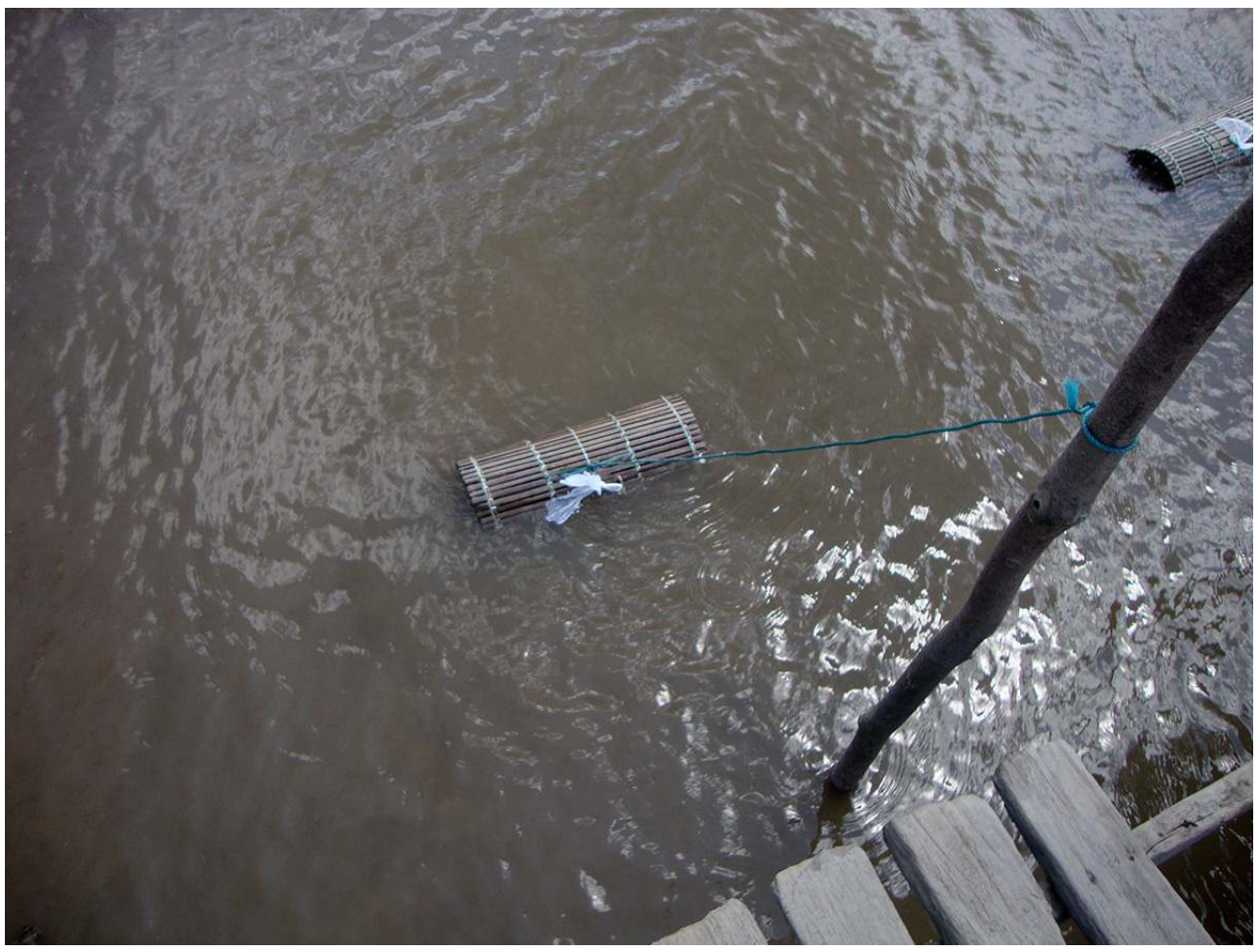

(c) P. Laval 
Figure 103 : Dona Dorialva sortant la nasse et vidant les crevettes sur l'Oyapock, village brésilien de Taparabo (Amapa)
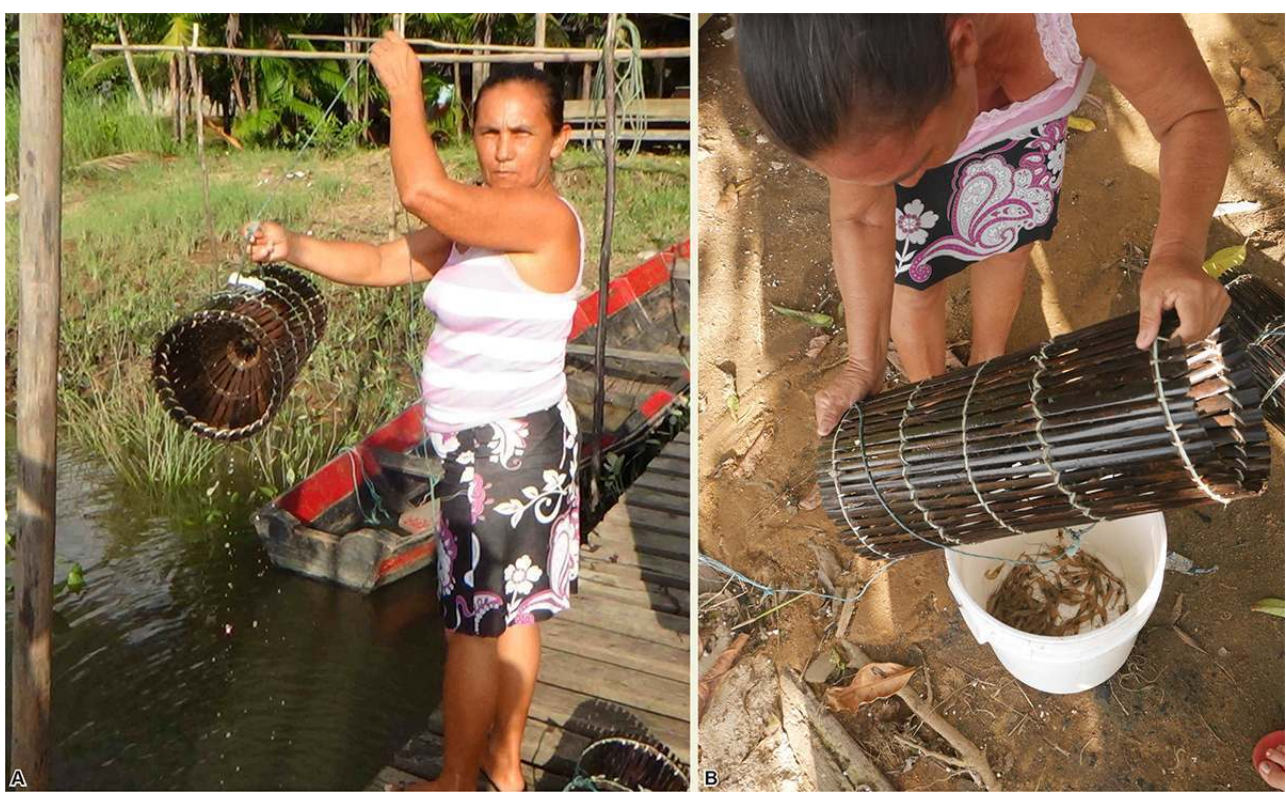

(C) A- P. Laval ; B- S. Bahuchet

215 - À double dispositif d'entrée

76) Deux nasses vietnamiennes

Figure 104 : nasses (Vietnam) ETB-VN-SBF-2016-001 et 002 - vue de profil avec la double entrée, de face et de dos montrant la porte coulissante.

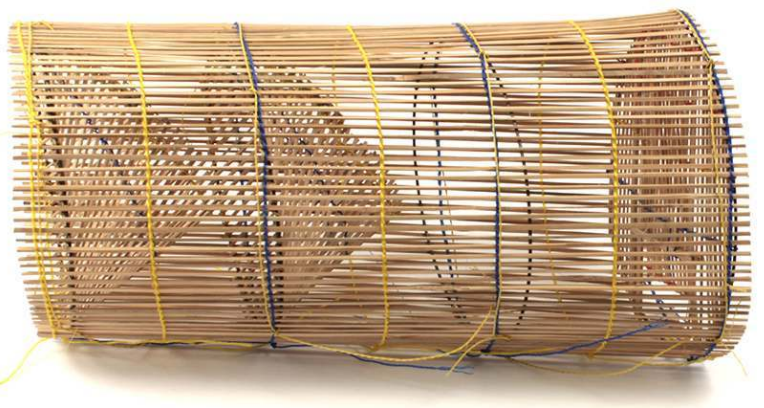

A-1<smiles>[Li][14C]#[14C]</smiles>

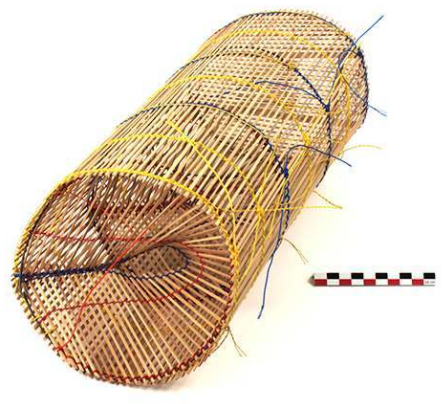

A-2 A. 3

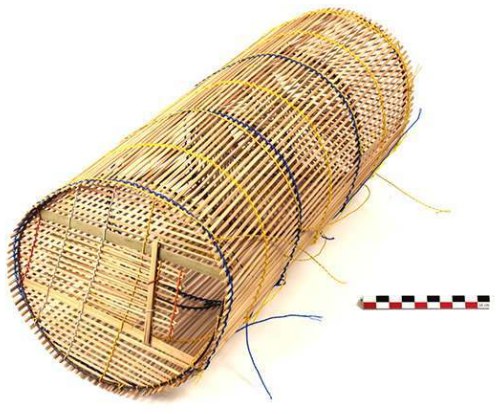

(C) P. Rameau 
217 • Vietnam ; village Binh Hòa Phuöc dans le delta du Mekong, Cấn Thơ, Province Vĩnh Long

- Nasses à double entrée pour les eaux douces. Tiges de bambou et fils de plastique. Une petite porte à coulisse à l'extrémité permet d'extraire les prises, petits poissons ou crevettes

- 07/2016 ; collectrice Sylvie Bahuchet-Fritsch

- Longueur $34 \mathrm{~cm}$, diamètre $15,5 \mathrm{~cm}$

- ETB-VN-SBF-2016-001 et 002

\section{7) Modèle réduit d'anguillère, nasse à anguille à deux chambres}

Figure 105 : Nasse à anguille à deux chambres ETB-FR-RP-2011-073

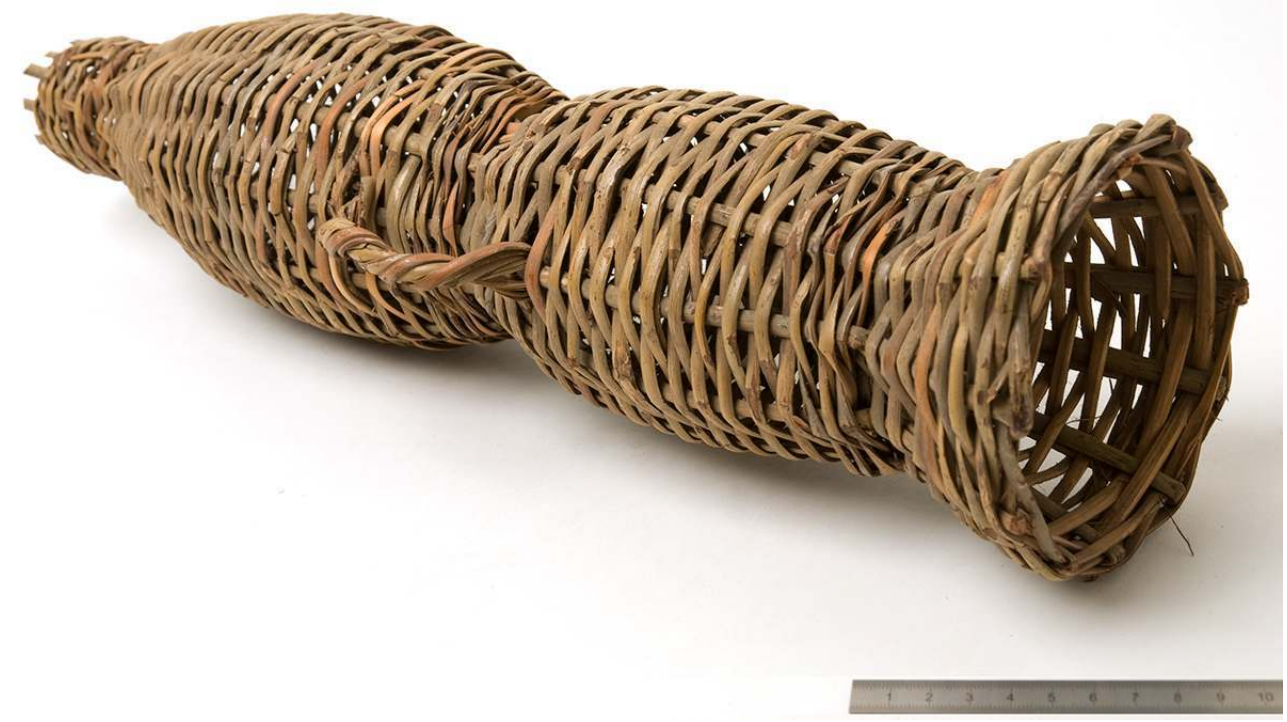

\section{(C) J.-C. Domenech}

219 - France ; Irleau-le Vanneau, marais poitevin, Deux-Sèvres

- En osier de vannier, Salix purpurea. Bourgnin, ou botte. Cette nasse est précédée d'un grand entonnoir, la heurte, augmentant la portée du goulet. Disposée lors du retrait de l'eau des marais, heurtes et bourgnins sont placés au milieu d'un barrage de pieux et de planches. La baisse des eaux suffisait à pousser les anguilles dans le piège; nul appât n'était nécessaire. Lorsque l'eau a quitté le marais, au printemps, c'est «la pêche à la lâche » pour laquelle les bourgnins appâtés de vers de terre, sont posés seuls, le soir, et relevés au petit matin (Fouchier \& Chevalier $1973: 16$ )

- Juin 1972 ; collecteurs Danièle Fouchier et Jean-Pierre Chevalier

- Longueur $42 \mathrm{~cm}$. (Modèle réduit de moitié de la taille normale 85 x $13 \mathrm{~cm}$ )

- ETB-FR-RP-2011-073 
Figure 106 : La nasse bourgnin avec son avancée la heurte, hors de l'eau et à la sortie d'un fossé
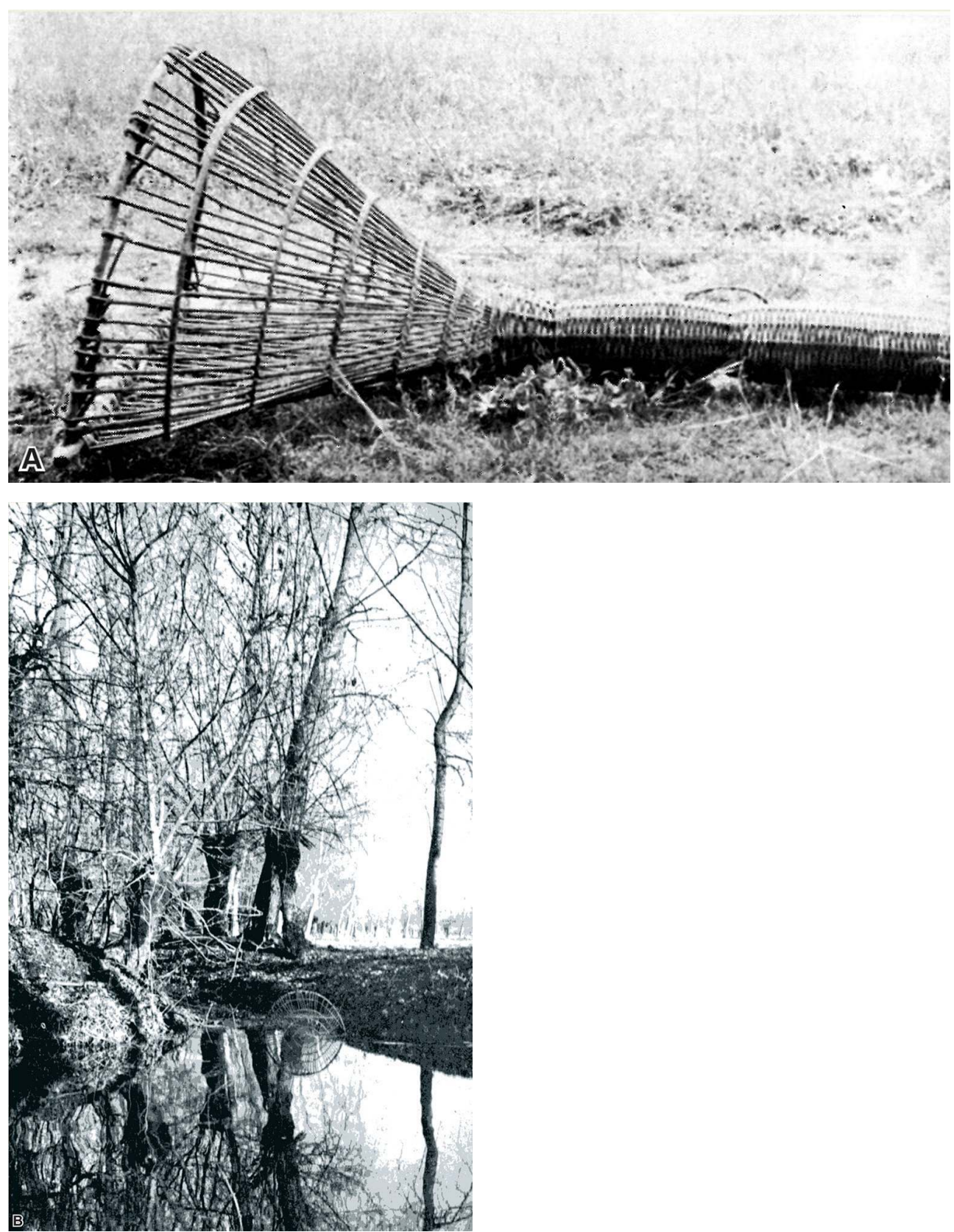

(c) A- D. Fouchier ;B- J.P. Chevalier (D'après Fouchier \& Chevalier 1973)

78) Nasse à anguille « Bourole» 


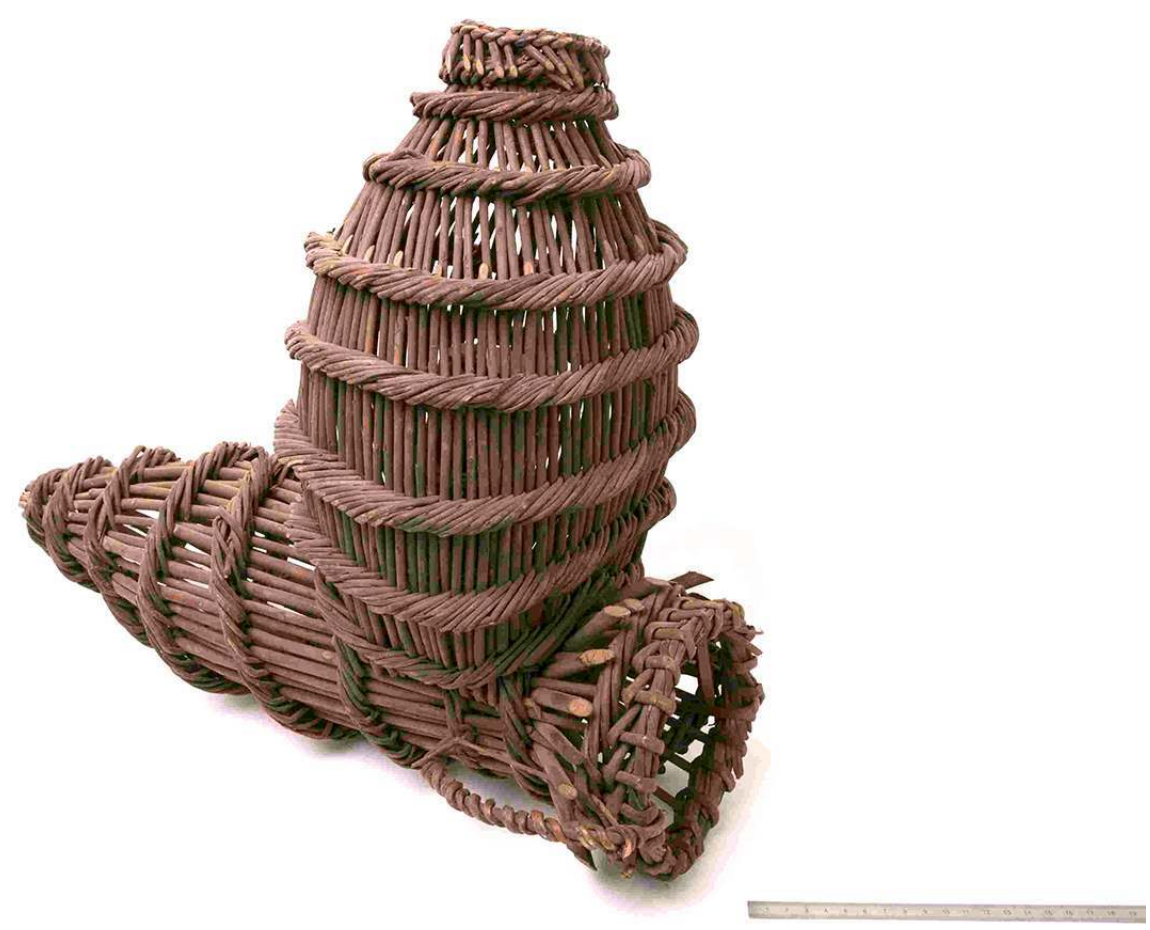

(C) J.-C. Domenech

221 - France; Marais poitevin, Vendée

- «Bourole». En osier jaune Salix alba. Un clapet en tôle à l'entrée de la $2^{\mathrm{e}}$ chambre. «C'était l'engin de pêche le plus caractéristique du marais poitevin. Appâtée avec des crevettes ou des moules, la nasse était placée dans le sens du courant pour piéger les anguilles. » (notice au Muséum de la Rochelle)

- Années 1970 ; collecteur Raymond Pujol

- Chambre horizontale : longueur $58 \mathrm{~cm}$, diamètre $15 \mathrm{~cm}$, hauteur totale $43 \mathrm{~cm}$, diamètre chambre du haut $19 \mathrm{~cm}$

- ETB-FR-RP-2011-074

\section{Tube, sans dispositif d'entrée}

L'étroitesse du tube empêche le poisson de se retourner pour fuir. Monod classe cet engin, décrit comme une "nasse tubulaire à entrée libre", dans la catégorie «emprisonnement (enceintes-pièges) » (p. 221).

Pour notre part, nous pourrions tout aussi bien l'inclure dans la catégorie "filtration ", $\mathrm{du}$ fait que c'est la force du courant qui pousse le poisson dans la nasse tubulaire, et l'y maintient - cependant Monod signale que «tous ces engins fonctionnent dans un courant » (p. 222). Nous en avions donné un exemple pour l'Afrique centrale (Bahuchet \& Rameau 2016, n²14).

79) Panier de capture tubulaire 


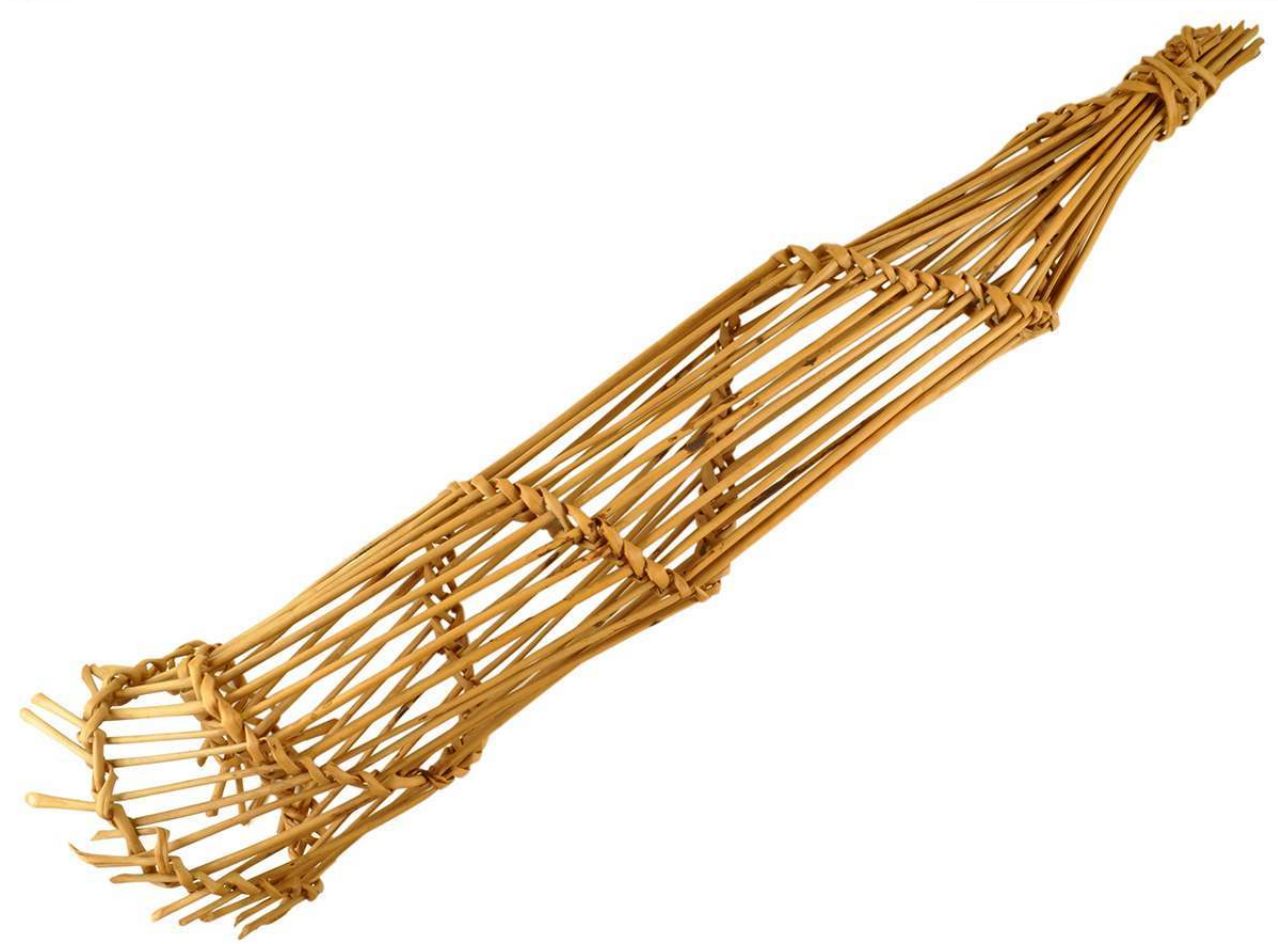

(C) D. Brabant

- Sans origine

- Nasse végétale constituée de 24 tiges d'osier réunies à la base par un lien pris progressivement dans une tresse qui monte en spirale vers le sommet

- Date de collecte inconnue ; ancienne coll. $\mathrm{MH}$, technologie comparée

- Longueur $52 \mathrm{~cm}$, diamètre de l'ouverture $12 \mathrm{~cm}$

- MNHN-E-2009.30.85

\section{Paralysie (Classe X)}

\section{Accessoires pour la pêche à la nivrée, Amazonie}

En Amazonie, la pêche à la nivrée (d'un terme créole dérivé d'énivré) est réalisée collectivement, aux basses eaux, à l'aide de plantes stupéfiantes dont les plus communes sont des lianes du genre Lonchocarpus (Fabacées), qui contiennent des roténones (Moretti \& Grenand 1982). Les plantes sont écrasées et plongées dans les cours d'eau, leur suc vénéneux s'écoule, provoquant la paralysie des organes respiratoires des poissons. Asphyxiés, ils flottent à la surface du cours d'eau, et sont recueillis par l'ensemble des assistants, hommes, femmes, enfants, avec des accessoires divers, épuisettes, flèches ou piques (cf. $\mathrm{N}^{\circ}$ 20). (Pour la Guyane, voir Pagezy \& Jegu 2004, 2010).

80) Filet Wuaorani 


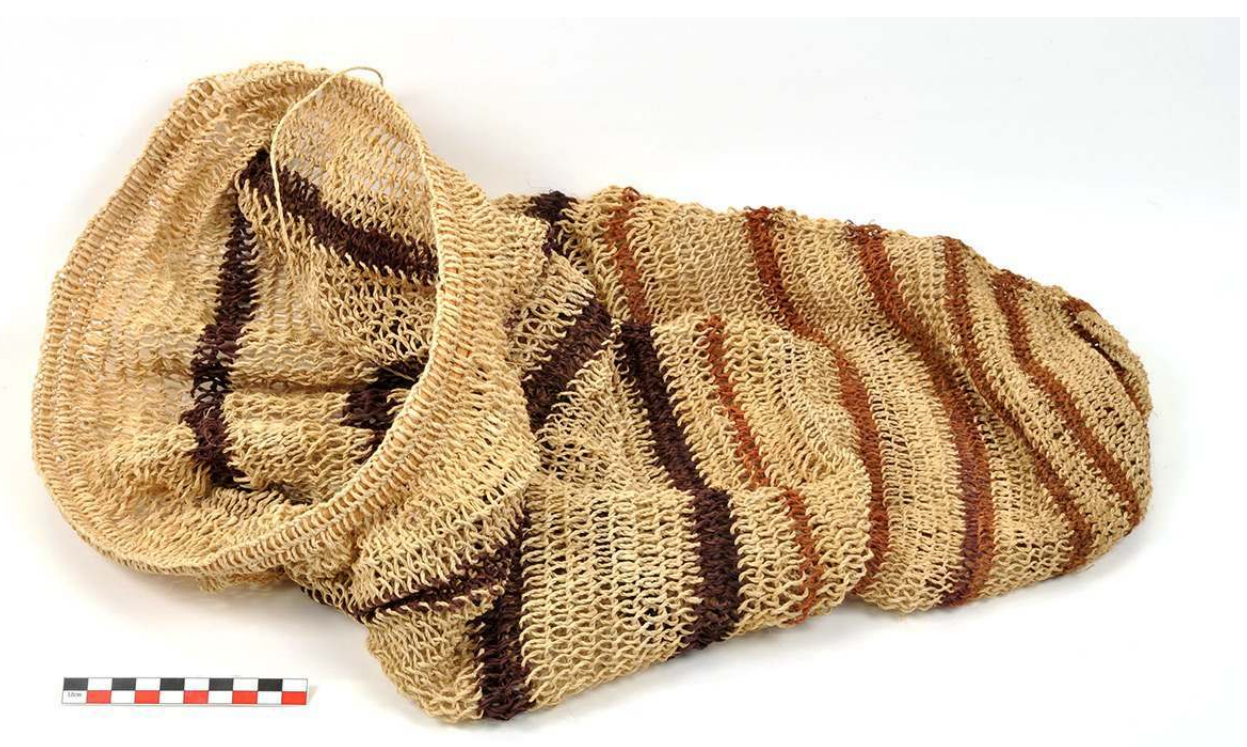

(C) P. Rameau

- Ethnie Waorani. Equateur ; village Tepapare, Provincia Pastaza

- Filet ruintai en fibres de folioles de palmier ongi (Astrocaryum chambira, Arécacées), utilisé lors de la pêche à la nivrée. La teinture rose est due à Guapira sp. (Nyctaginacées), la mauve à Picramnia sellowii (Simaroubacées). Il est manipulé à la main grâce au cercle rigide. Riuntai est le terme employé par les anciens, les jeunes nomment les épuisettes yeye digentai (" poisson-sacoche»), ce qui correspond peut-être aussi à un changement de forme, comme le montrent les photos ci-dessous. La pêche à la nivrée yeye yemongi (littéralement "poisson-lavage ») se pratique souvent dans les ruisseaux interfluviaux. Les pêcheurs pilent des racines de la liane Lonchocarpus spp. (Fabacées) ou celles de Clibadium sp. (Astéracées) et les enserrent dans des paniers; ils plongent en amont, les paniers qui exsudent la sève toxique, puis suivent le cours d'eau. Le suc asphyxie les poissons, qui flottent alors la surface. On les assomme avec le manche de la machette et on les récupère avec le panier-épuisette (Zurita 2014 : 105-106)

-12/2012 ; collectrice Maria Gabriela Zurita

- Diamètre $24 \mathrm{~cm}$, longueur $57 \mathrm{~cm}$

- ETB-EC-GZB-2012-005 
Figure 110 : Une nivrée à Tepapare, en mars 2010
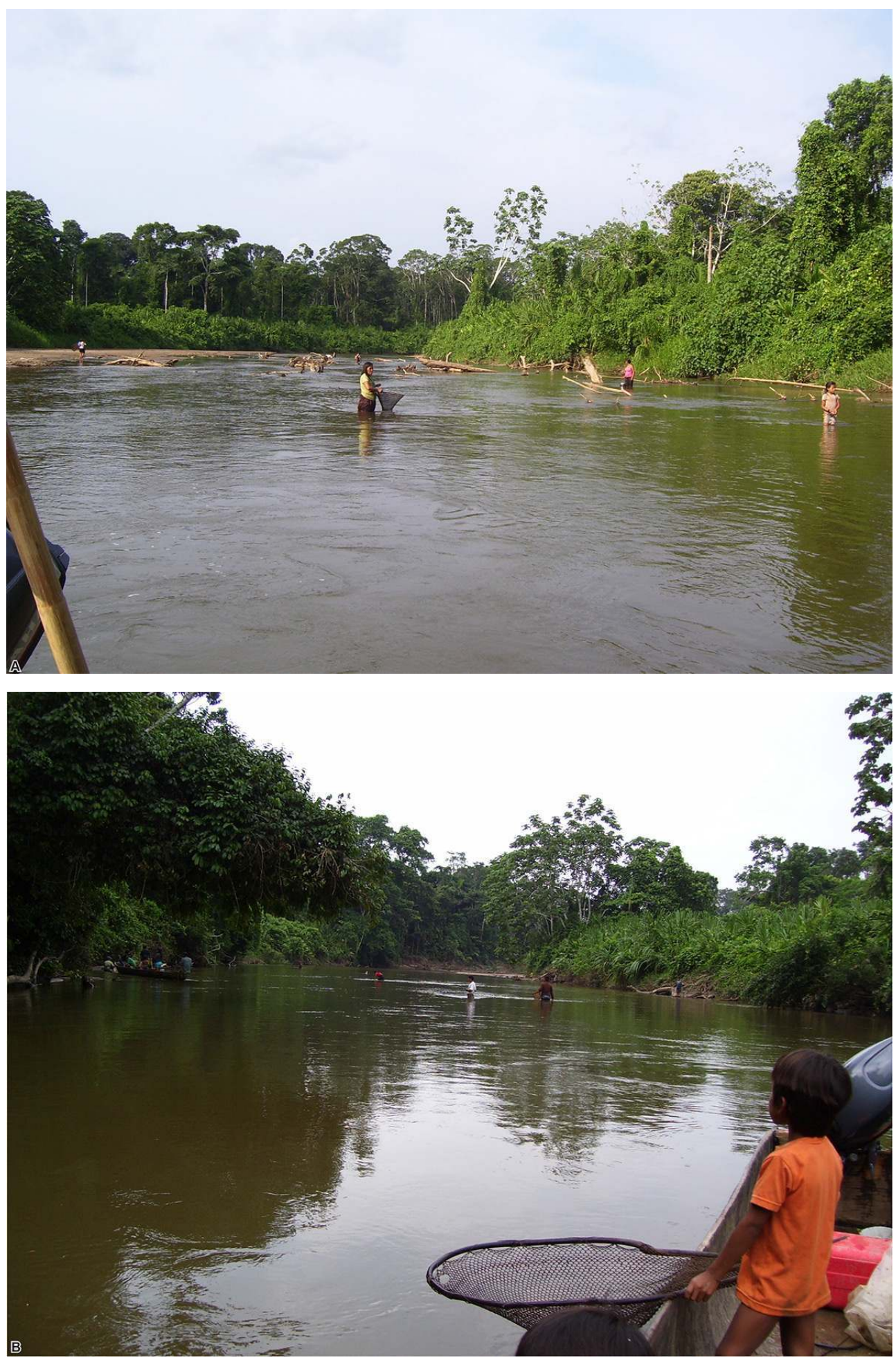

(c) G. Zurita

81) Panier-épuisette wayana 


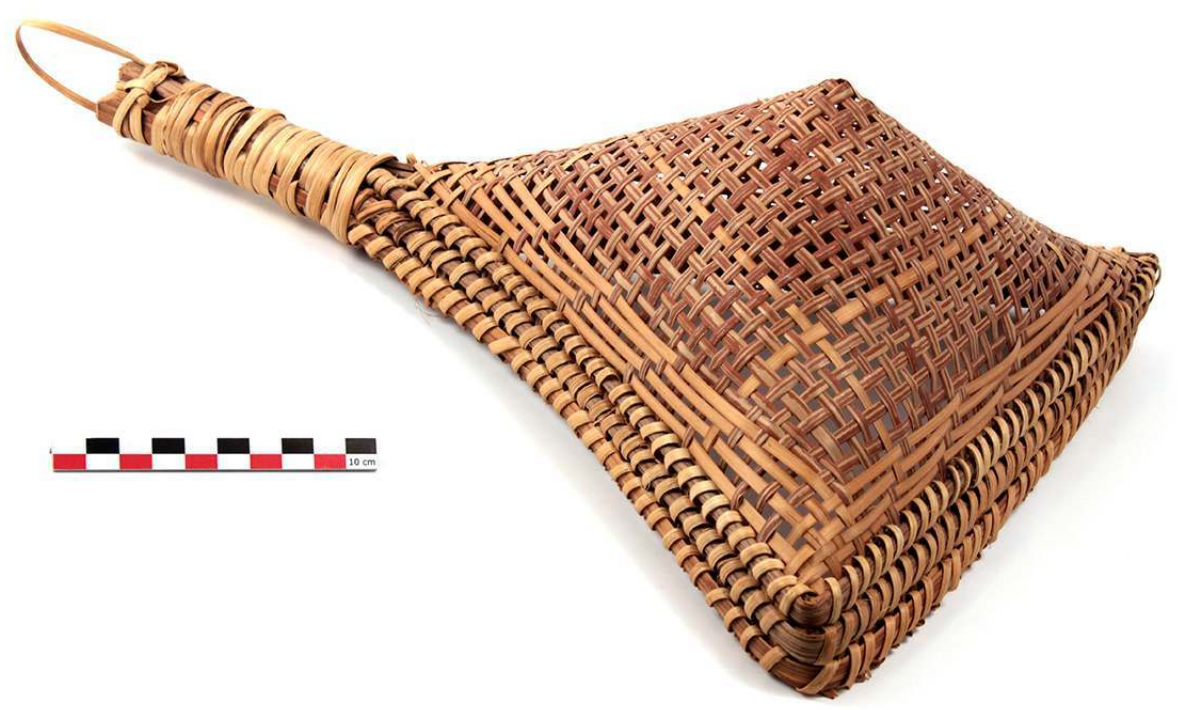

(C) P. Rameau

- Ethnie Wayana. Guyane française ; Antecume Pata, Haut Maroni, ; acquis au cours de la mission « Nivrée 2000 »

- Panier-épuisette triangulaire pilipa ; se fixe sur un long manche. Permet de capturer les poissons étourdis lors de la pêche collective à la nivrée. Cette épuisette est surtout maniée par les femmes (Davy 2007 : 179)

- Oct-nov. 2000 ; collectrice Hélène Pagezy

- Longueur $36 \mathrm{~cm}$, largeur $26 \mathrm{~cm}$, hauteur $12,5 \mathrm{~cm}$

- ETB-GF-HP-2000-008 
Figure 112 : Battage des tiges de la liane Lonchocarpus, dont la sève va enivrer les poissons

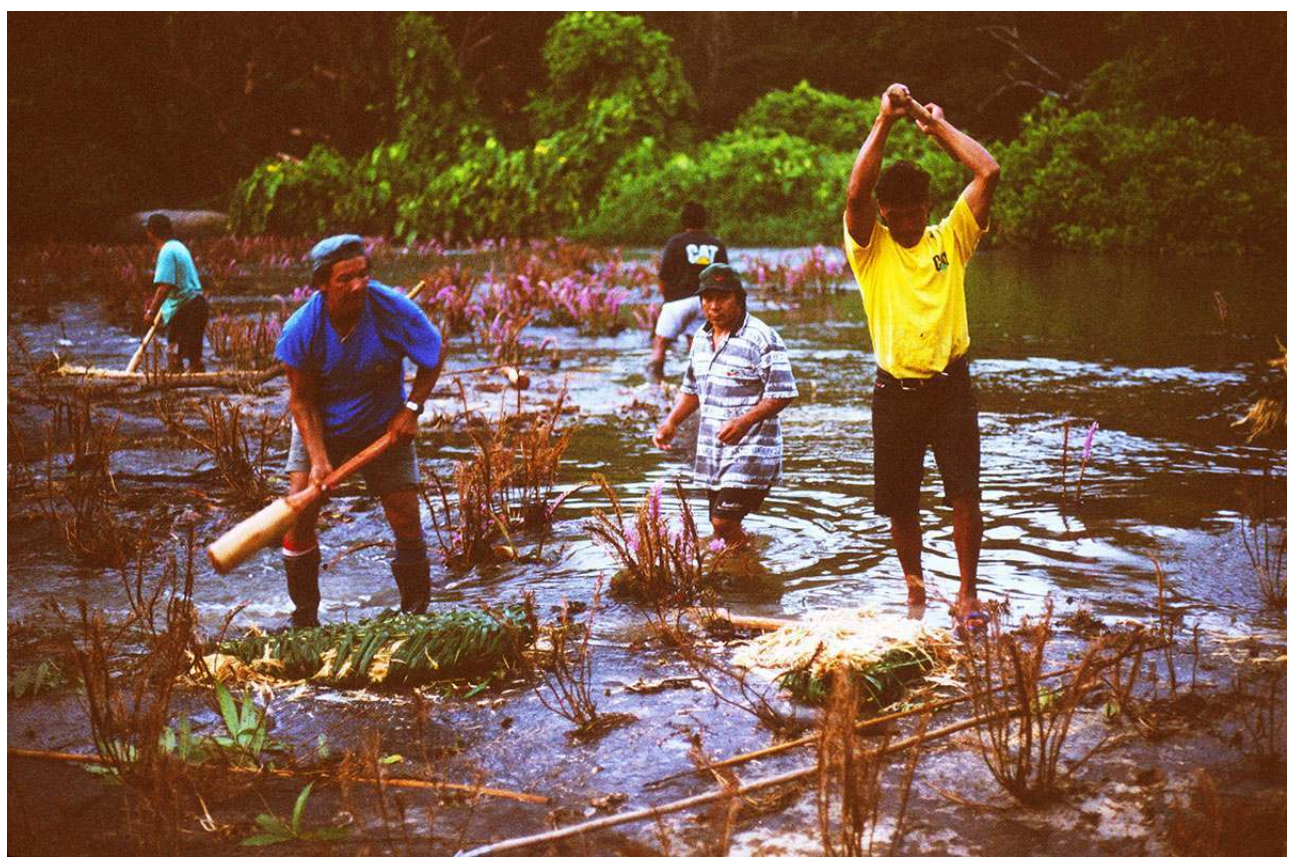

(c) H. Pagezy

Figure 113 : Vue d'ensemble de la nivrée dans les sauts du Haut Maroni, aux basses eaux

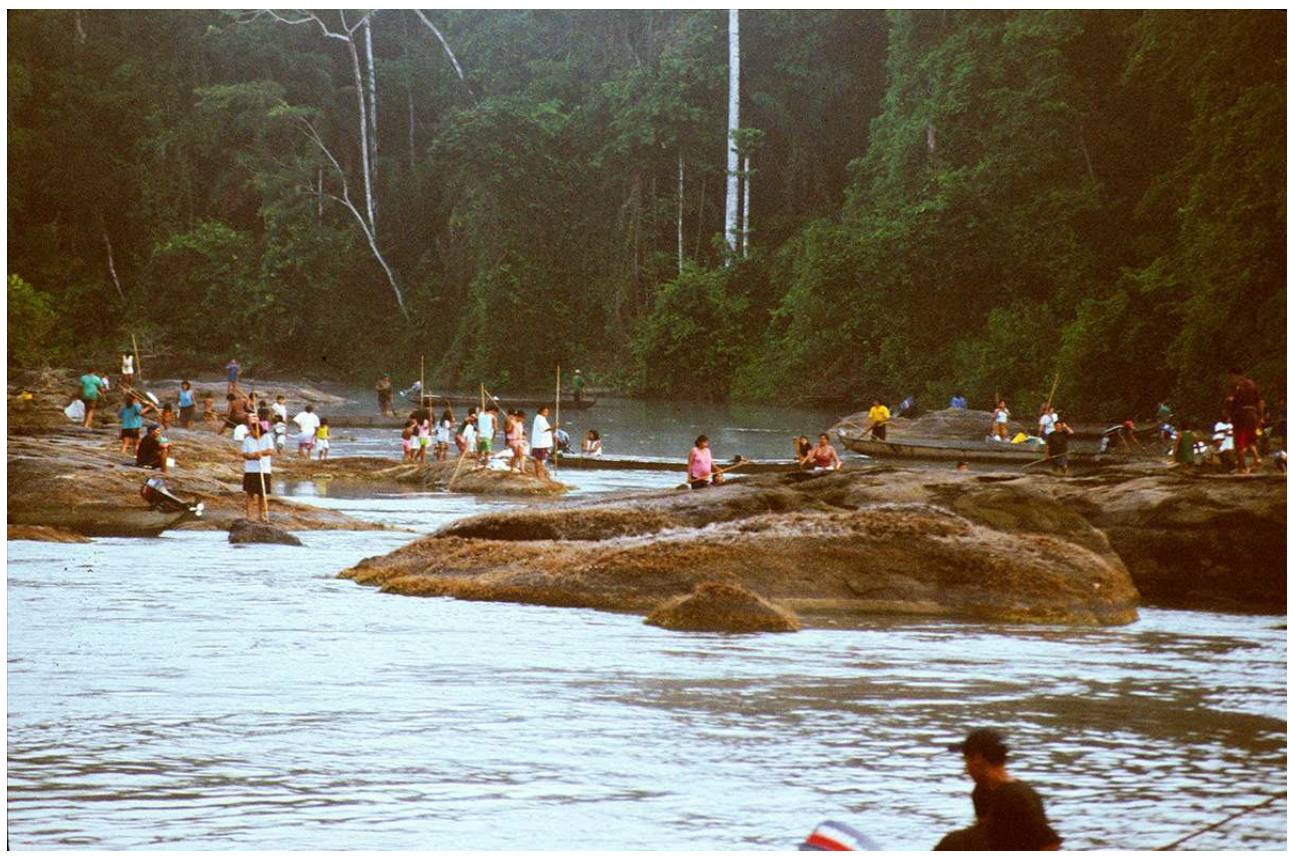

(c) H. Pagezy 
Figure 114 : Dessin d'enfant représentant le fléchage des poissons qui flottent à la surface, pendant la nivrée

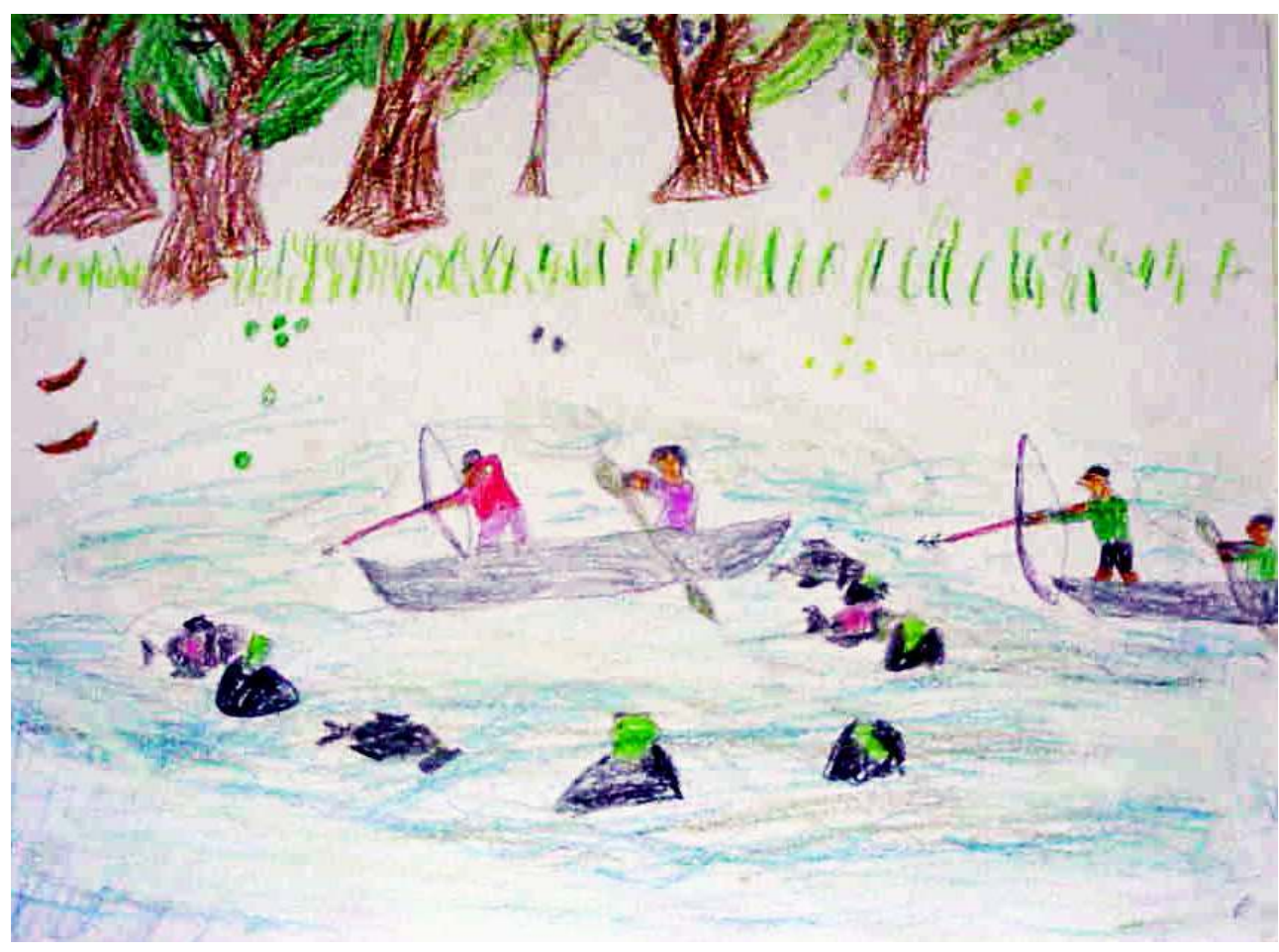

〔 Dessin réalisé par Maurice et recueilli par Hélène Pagezy à Antecoume Pata.

Déterrement-Ramassage (Classe XII)

Ramasser, détacher du substrat

Mytiliculture

82) «Pêchoire », grattoir-épuisette pour récolter les moules 


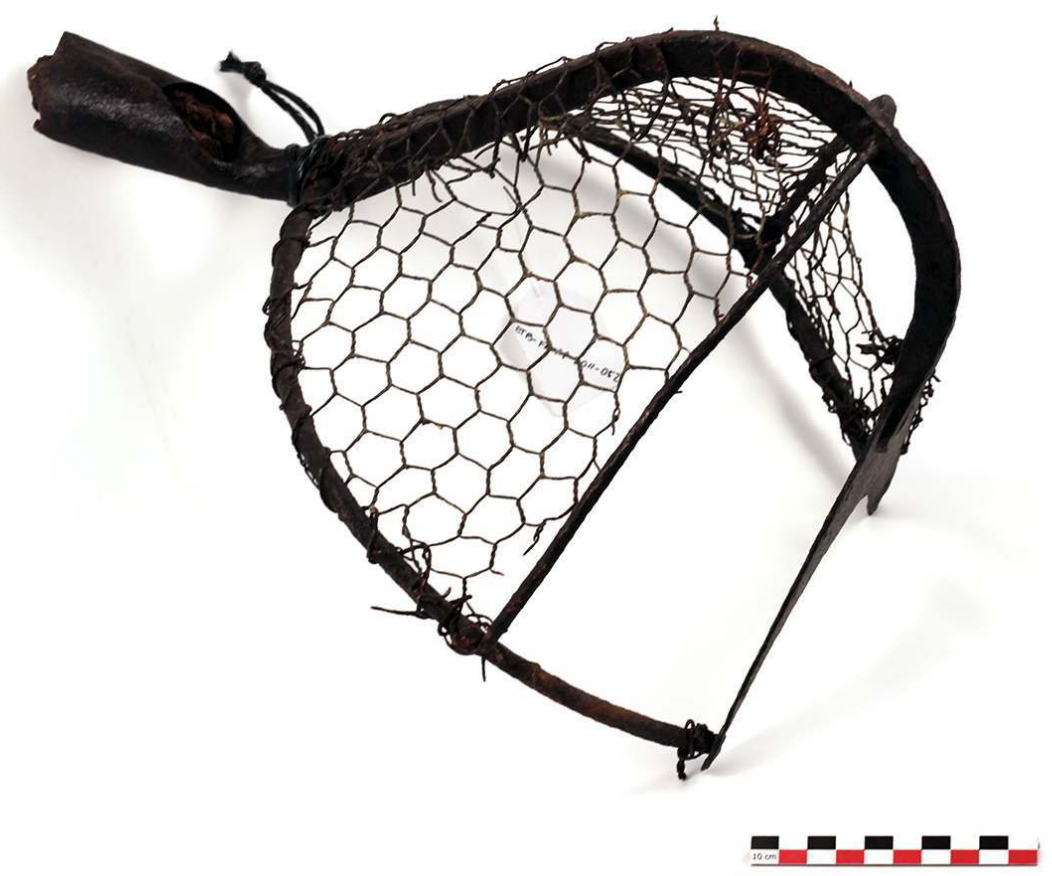

(c) S. Bahuchet

- France ; La Tranche sur mer, Vendée

- «Pêchoire ", grattoir-épuisette pour récolter les moules, en fer forgé, poche en grillage métallique. Se fixe sur un long manche. L'usage de ces ustensiles a été abandonné dans les années 70

- Années 1970 ; collecteur Raymond Pujol

- $30 \times 32 \times 25 \mathrm{~cm}$

- ETB-FR-RP-2011-052

\section{Ostréiculture}

83) Couteau " détroqueur» 


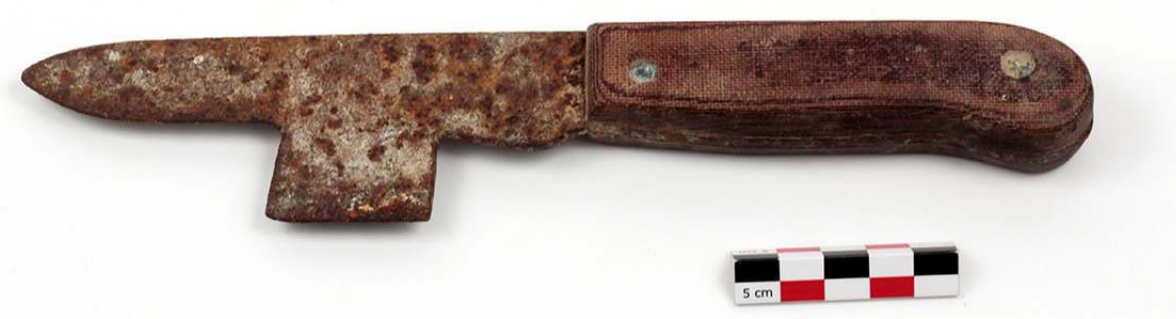

(c) S. Bahuchet

234 - France ; bassin d'Arcachon, Gujan-Mestras, Gironde

- Couteau détroqueur, pour détacher les amas d'huîtres. Don d'un ostréiculteur

- Février 2012 ; collectrices Carole Delamour et Nastassia Reyes

- $25 \times 5 \times 2,5 \mathrm{~cm}$

- ETB-FR-CDNR-2012-002

84) Couteau « déchauleur»

Figure 117 : ETB-FR-CDNR-2012-001

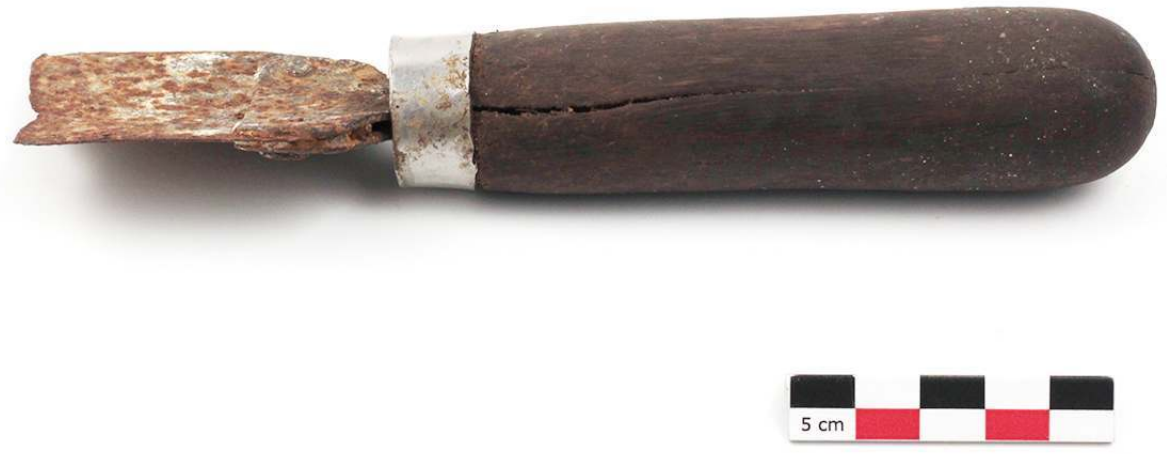

(c) S. Bahuchet

236 - France ; bassin d'Arcachon, Gujan-Mestras, Gironde

- Couteau "déchauleur », utilisé pour racler la chaux présente sur les tuiles servant à collecter le naissain des huîtres. La technique du chaulage des tuiles, inventée en 1865, permet de détacher les jeunes huîtres des tuiles, sans les abimer. Don d'un ostréiculteur - Février 2012 ; collectrices Carole Delamour et Nastassia Reyes

- 13 x $5 \mathrm{~cm}$, diamètre du manche $3 \mathrm{~cm}$

- ETB-FR-CDNR-2012-001 
Figure 118 : Les tuiles enduites de chaux devant la cabane de l'ostréiculteur, avant la mise à l'eau

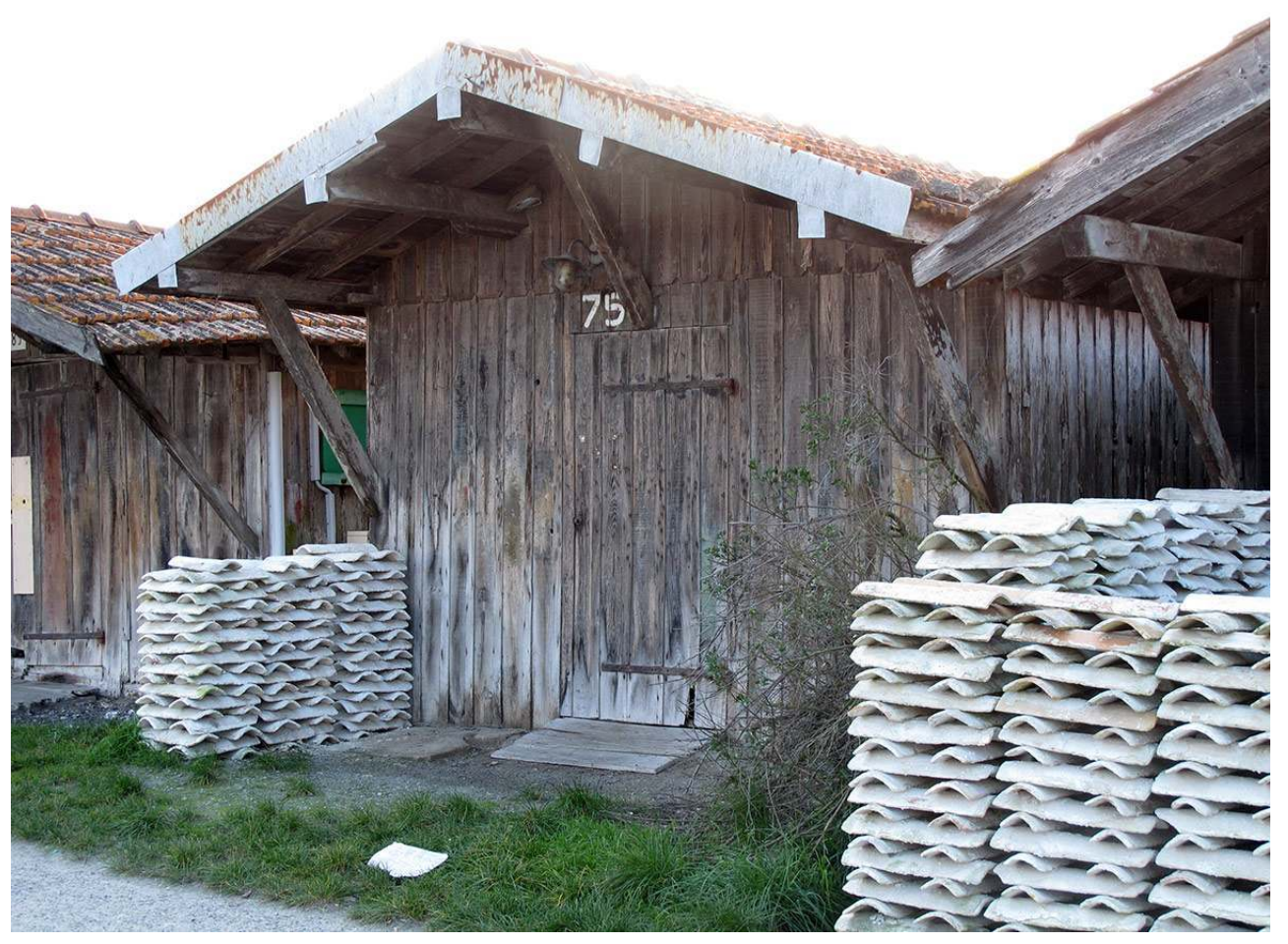

(c) S. Bahuchet

237 85) Coupelles pour collecter le naissain des huitres

Figure 119 : Coupelles pour le naissain des huîtres ETB-FR-SB-2018-006

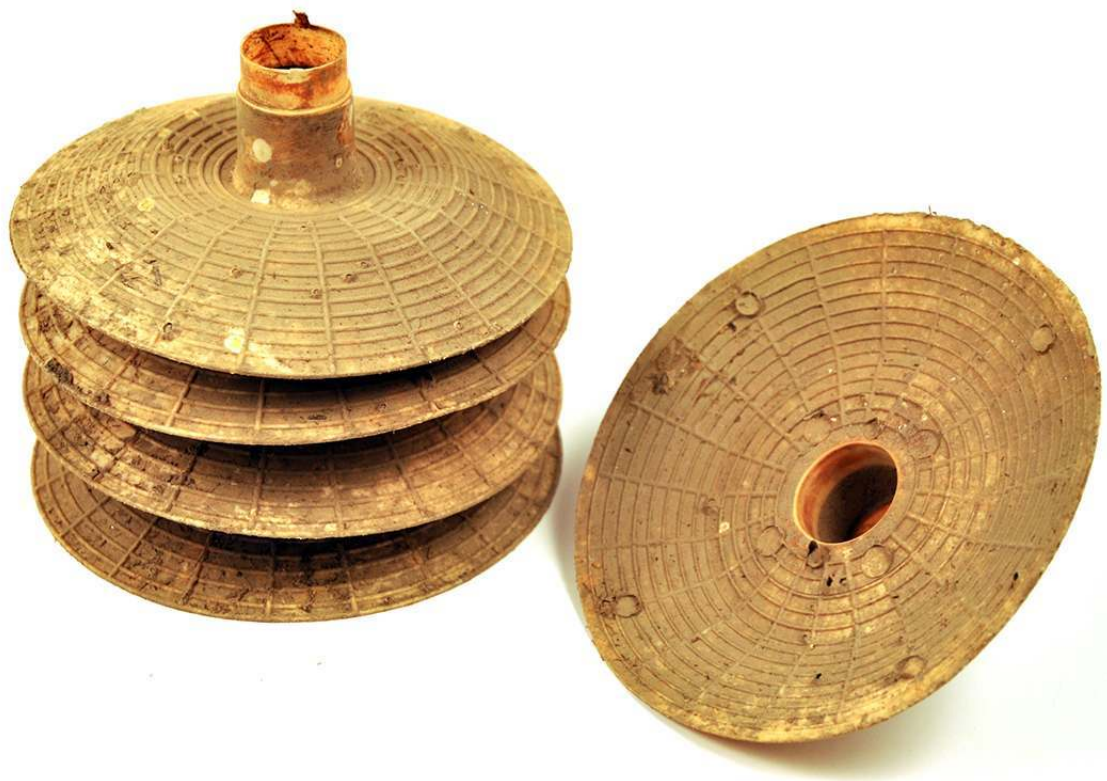

(C) S. Bahuchet 
- France ; bassin d'Arcachon, Gujan-Mestras, Gironde

- Coupelles en matière plastique pour collecter le naissain des huîtres

- Février 2012 ; collecteur Serge Bahuchet

- Diamètre $14 \mathrm{~cm}$, hauteur d'un disque $4 \mathrm{~cm}$

- ETB-FR-SB-2018-006

Figure 120 : Coupelles avant la mise à l'eau

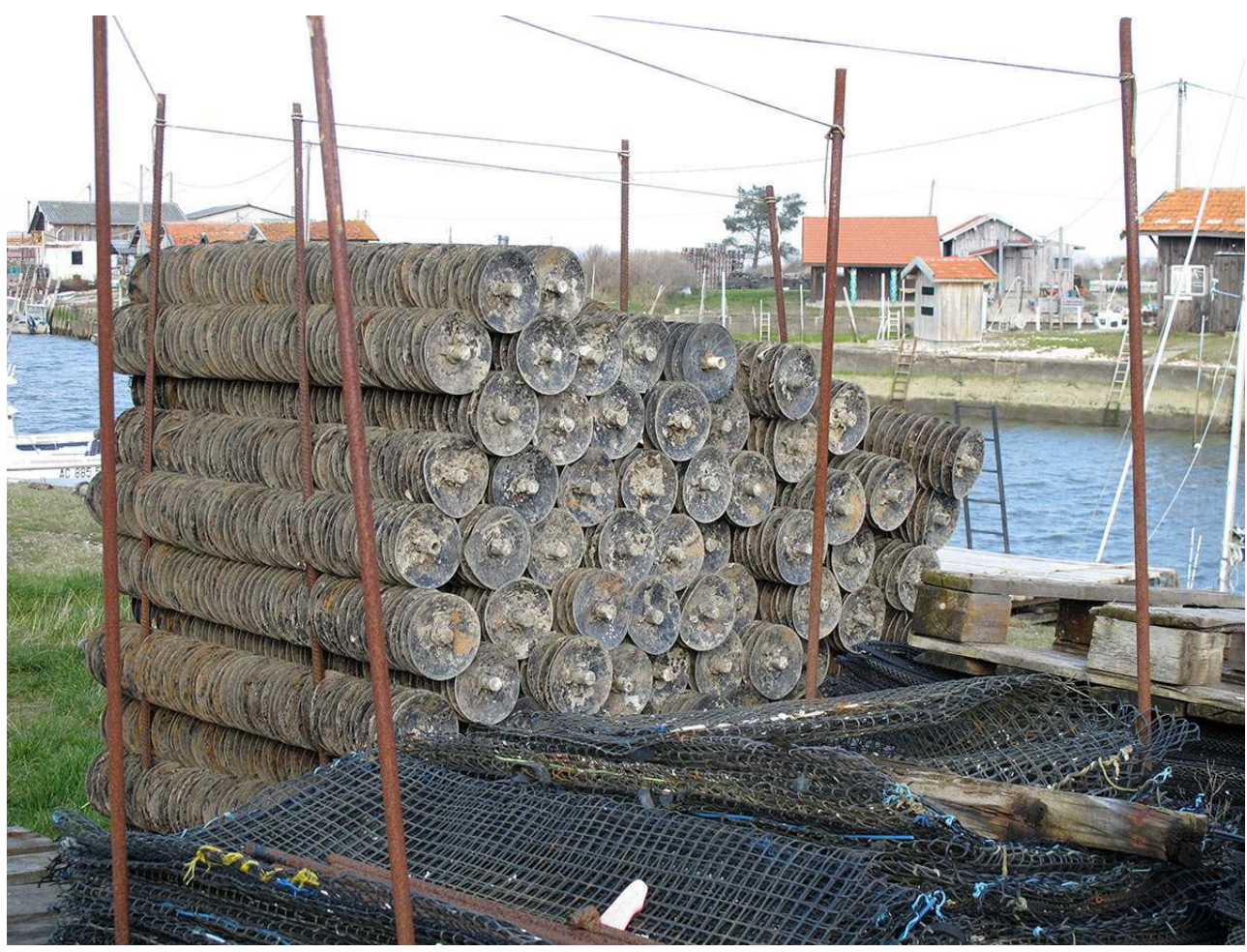

(c) S. Bahuchet

Déterrement (dans le sol)

86) Grand tellinier sicilien 

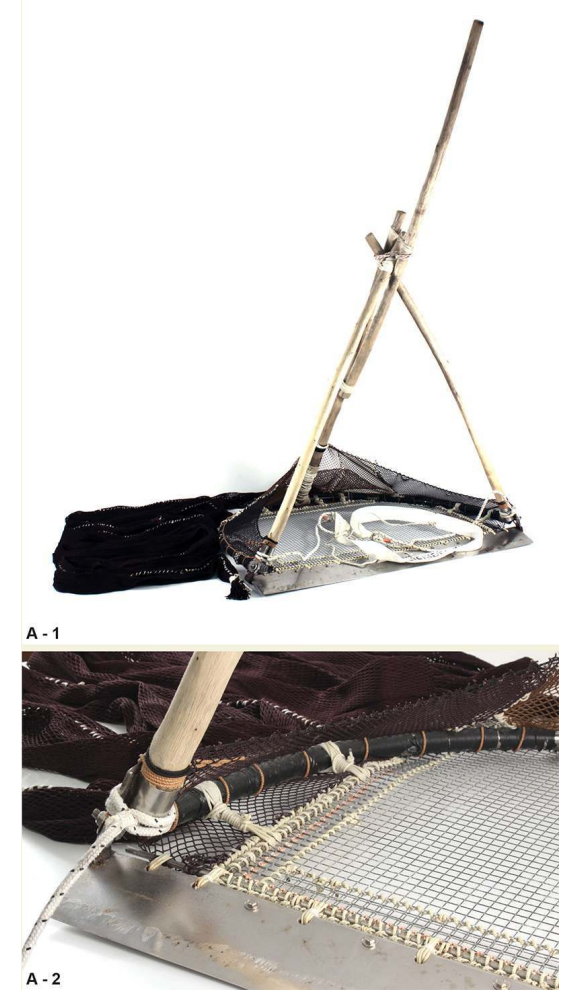

(C) P. Rameau

\section{- Italie ; Catane, Sicile}

- Cet engin des pêcheurs à la piccola pesca de Sicile, est destiné à la pêche aux tellines ( Donax trunculus). Ce mollusque bivalve est présent dans les fonds sableux et s'enlise jusqu'à 2 à 3 mètres de fond. Dans la province de Catane, la pêche de la telline est réalisée par de pêcheurs artisans appelés cuzzularu en dialecte catanais. Le râteau-tellier, rastrello per le telline en italien, est un outil essentiellement artisanal, souvent construit et adapté par les pêcheurs eux-mêmes. Il est formé d'une structure, ici en bois, reliée à une plate-forme de mailles métalliques destinée à être trainée sur le sol. A l'avant se trouve une lame d'acier appelée peigne, tandis qu'à l'arrière une poche constituée d'un filet de pêche permet de récupérer les tellines. Cet engin de pêche a été recueilli auprès d'Agostino Calogero, ancien fonctionnaire de la capitainerie de Catane

- 2015 ; collectrice Nastassia Reyes

- Largeur de la base $98 \mathrm{~cm}$, profondeur $67 \mathrm{~cm}$; hauteur du manche $155 \mathrm{~cm}$; longueur du filet $412 \mathrm{~cm}$

- ETB-IT-NR-2015-004

La pêche aux tellines est effectuée manuellement, par un pêcheur qui, debout dans l'eau, active le manche central en marchant à reculons. Ce faisant il effectue une traction plus grande et uniforme de l'outil pour labourer le fond sablonneux (Figure 122B). Pendant la traction, le pêcheur secoue le râteau d'avant en arrière et fait pénétrer l'engin sous une profondeur de 15 à $20 \mathrm{~cm}$ dans le sable, permettant ainsi aux tellines situées les plus à la surface d'affleurer. C'est une activité physique très fatigante. Elle est pratiquée dans des fonds allant jusqu'à un mètre vingt. Au-delà de cette profondeur, la pêche de la telline peut être effectuée à l'aide d'un bateau. Dans ce cas, un outil à dent est aussi utilisé mais 
de plus grande taille et avec une tige de remorquage plus longue. Sur la zone ionienne, ce type de pêche est une activité artisanale, encore pratiquée aujourd'hui, qui n'utilise pas d'équipement mécanique.

Figure 122 : Pêcheur de telline
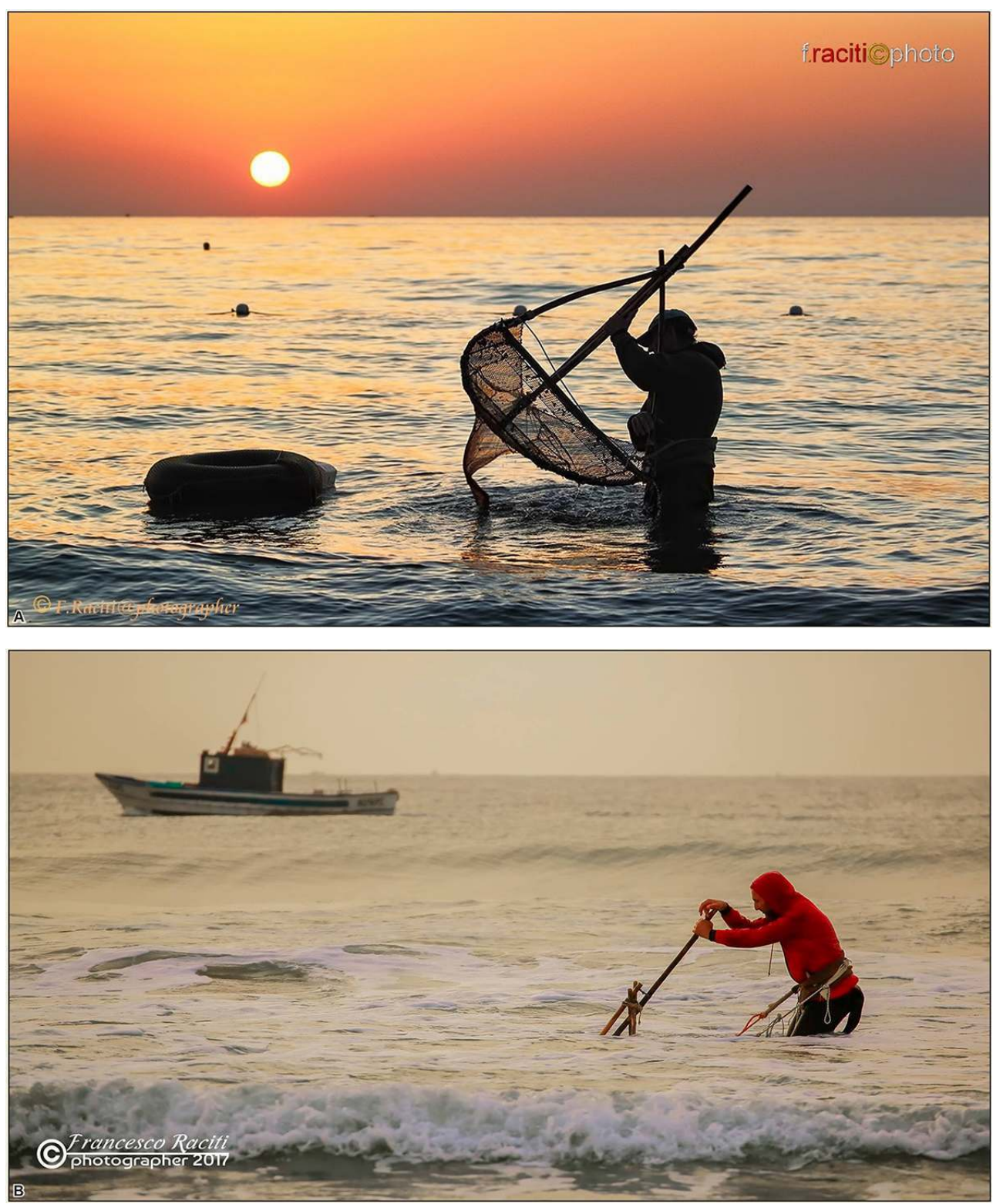


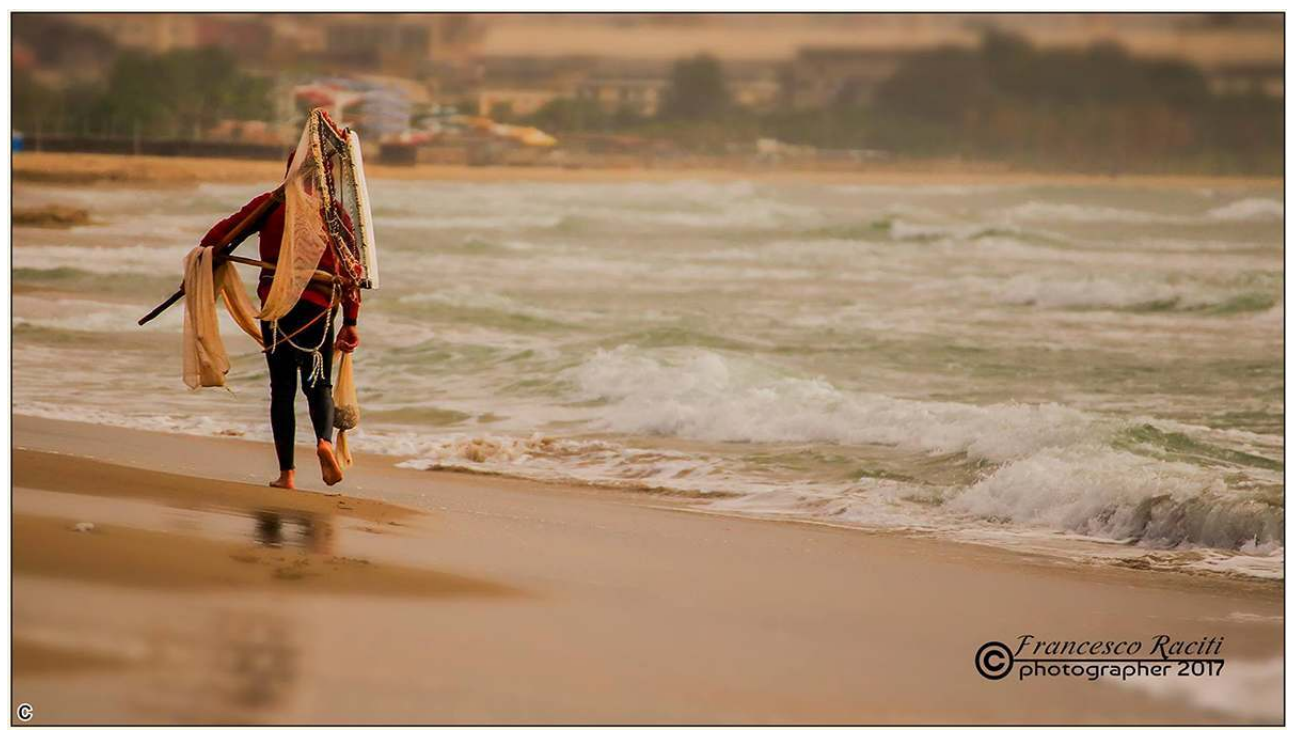

(c) F. Raciti, Catane

\section{7) Panier à nettoyer les coquillages}

Figure 123 : Panier à nettoyer les coquillages
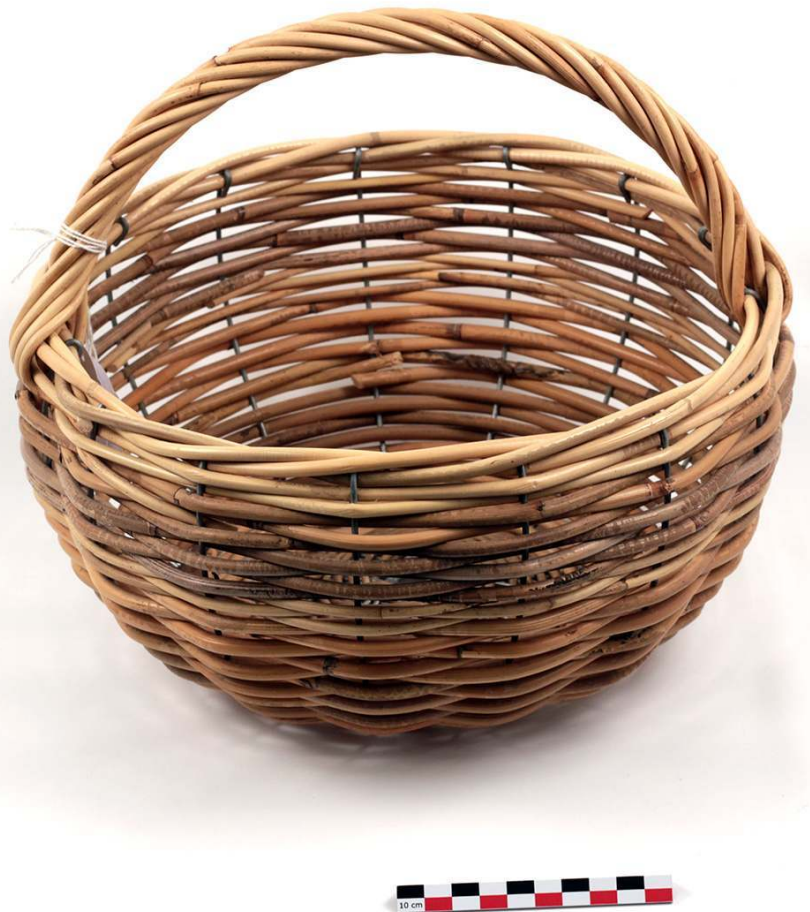

(c) S. Bahuchet

- France. Bretagne, Tréboul, Finistère. Achat au marché

- «baz koden ». En rotin d'Asie, sur une armature de fil de fer. On le plonge dans l'eau pour nettoyer le sable des coquillages. Fait par Robert Lossouarn, de Pont Labbé. (Hérisset $2014: 109)$.

- Août 2006 ; collectrice Jeanne Le Duchat 
- Diamètre $29 \mathrm{~cm}$, hauteur totale $25 \mathrm{~cm}$, hauteur du panier $17 \mathrm{~cm}$

- ETB-FR-JLD-2006-001

Réglementation

En France la collecte par des amateurs des coquillages, sur les plages, est autorisée mais soumise à une réglementation portant sur la période, sur la taille des individus prélevés, et la quantité par ramasseur.

88) Gabarit pour crustacées et coquillages

Figure 124 : gabarit « crabe-toiseur » ETB-FR-SB-2016-001

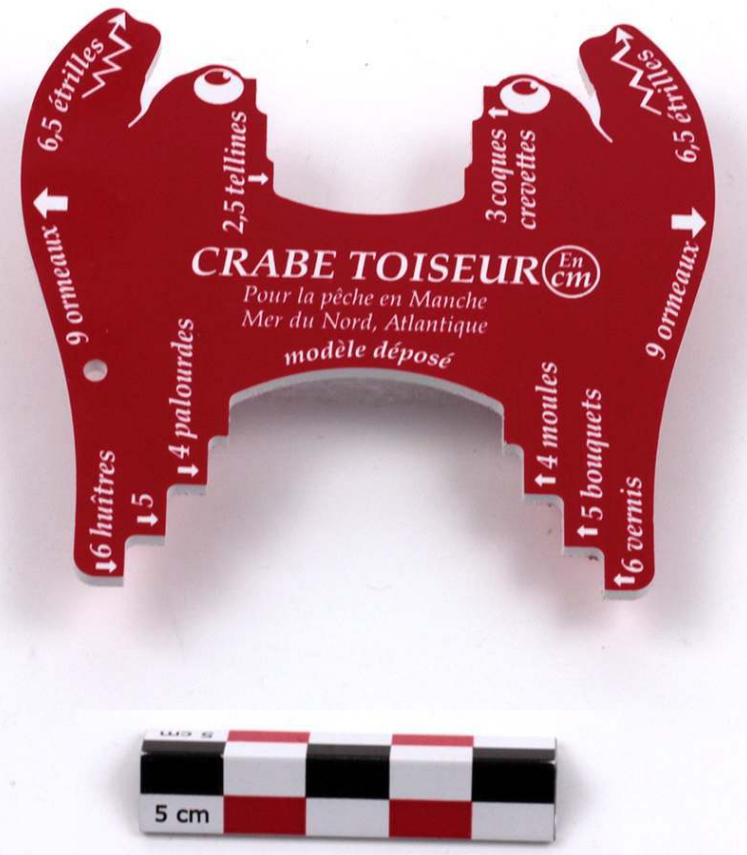

(c) S. Bahuchet

246 • France ; Erquy, Côtes d'Armor ; Comptoir de la mer

- «Crabe toiseur » en matière plastique ; pour la région Atlantique nord, marque Beastuce (29120 Combrit). Découpé en forme de crabe, des échancrures dans la plaque de plastique permettent de mesurer des crustacées (crevettes, bouquets, étrilles) et des coquillages (huîtres, moules, palourdes, ormeaux, coques, vernis et tellines)

-07/2016; collecteur Serge Bahuchet

- 9 × $8,2 \mathrm{~cm}$

- ETB-FR-SB-2016-001

89) Gabarit pour crustacées et coquillages 

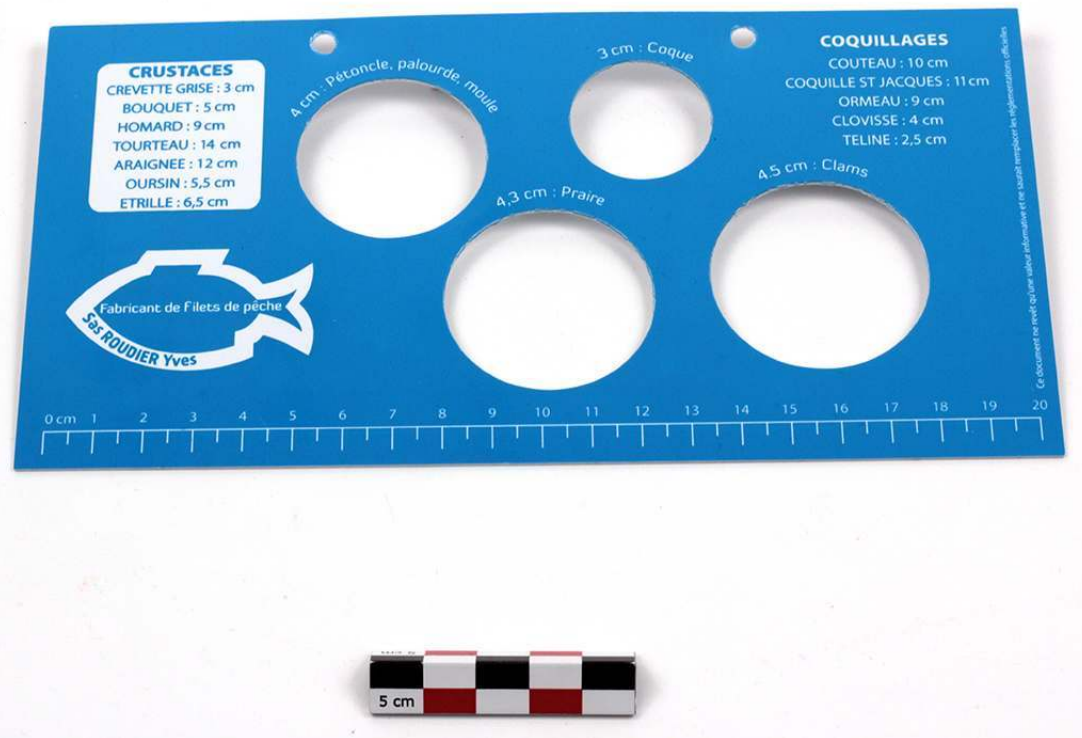

(c) S. Bahuchet

- France ; Erquy, Côtes d'Armor ; Comptoir de la mer

- "Plaquette calibreur", gabarit pour crustacées et coquillages en matière plastique. Marque SaS Roudier Yves (17120 Brie-Sous-Mortagne; Charente Maritime). Plusieurs ouvertures permettent de vérifier la taille des coquillages (pétoncles, coques, clams, praires). Une règle de $20 \mathrm{~cm}$ permet de mesurer les poissons

- 07/2016 ; collecteur Serge Bahuchet

- $21 \times 11 \mathrm{~cm}$

- ETB-FR-SB-2016-002

90) Panier-calibreur pour la pêche à pied 


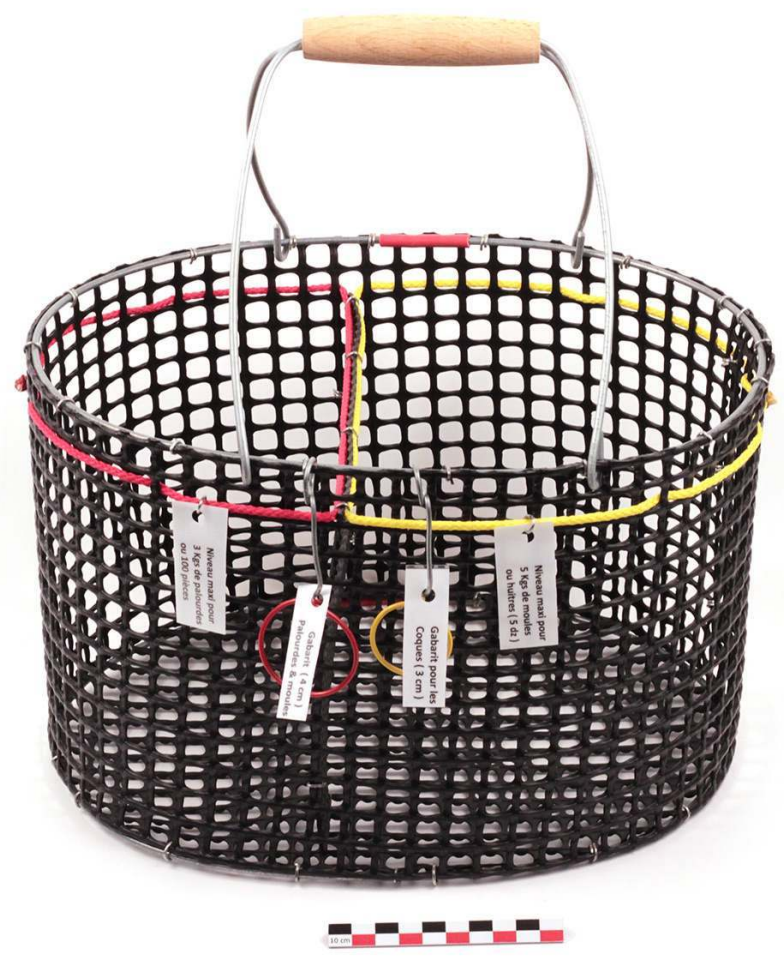

(c) S. Bahuchet

- France ; Bretagne ; Achat au marché de Dinard

- Panier-calibreur pour la pêche à pied des coquillages. Inventé par Mohamed Teffahi (St Nazaire, Loire-Atlantique). Fabriqué avec des poches à huîtres recyclées. Ce panier composite est constitué de deux parties inégales, l'une pour mesurer $3 \mathrm{~kg}$ de palourdes, l'autre pour cinq douzaines d'huîtres ou de moules. Les deux anneaux suspendus sur le côté sont des gabarits pour vérifier la taille réglementaire des coques $(3 \mathrm{~cm})$ ou des moules et palourdes $(4 \mathrm{~cm})$

- 09/2016 ; collectrice Sylvie Le Bomin

- 33 x 23,5 cm, hauteur du panier $21 \mathrm{~cm}$, hauteur totale $36 \mathrm{~cm}$

- ETB-FR-SLB-2016-001

\section{Après la pêche}

Assommer le poisson

91) Massue de pêcheur 


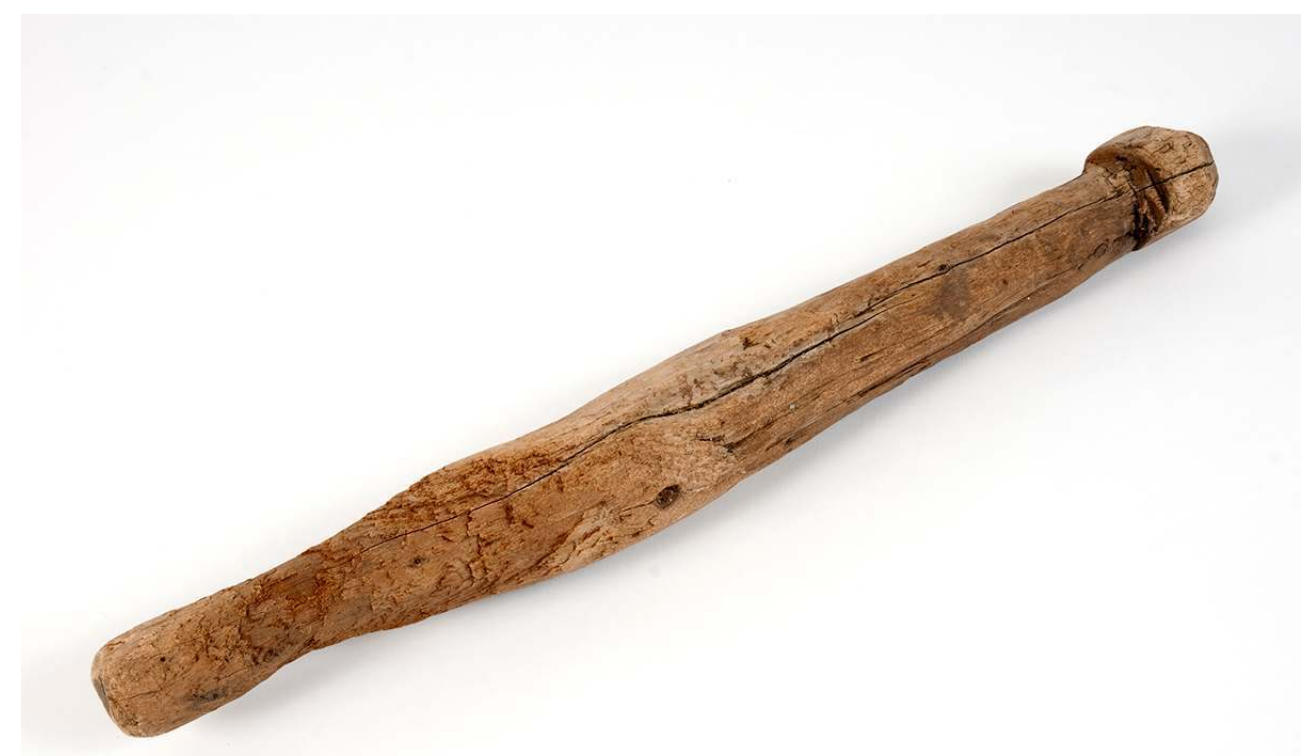

(c) MNHN-Pôle multimédia

252 - République Dominicaine ; Village de Buen Hombre, Région de Monte Cristi. Trouvée au fond de l'eau, lors d'une plongée (du fait de la forte densité de ce bois) sous les bateaux de pêche

- Massue de pêcheur macana (langue taïno) en bois de manglier monoxyle aminci au niveau du manche et renflée à son extrémité. Destinée à la pêche aux poissons (barracuda...) ou à l'ouverture des coquilles de lambis pour en extraire le mollusque - Juin-juillet 2010 ; collecteur François Gendron

- Longueur $41 \mathrm{~cm}$, diamètre max. $5 \mathrm{~cm}$

- MNHN-E-2010.3.1

Préparation des prises

92) Couteau « Piqueur » à morue 


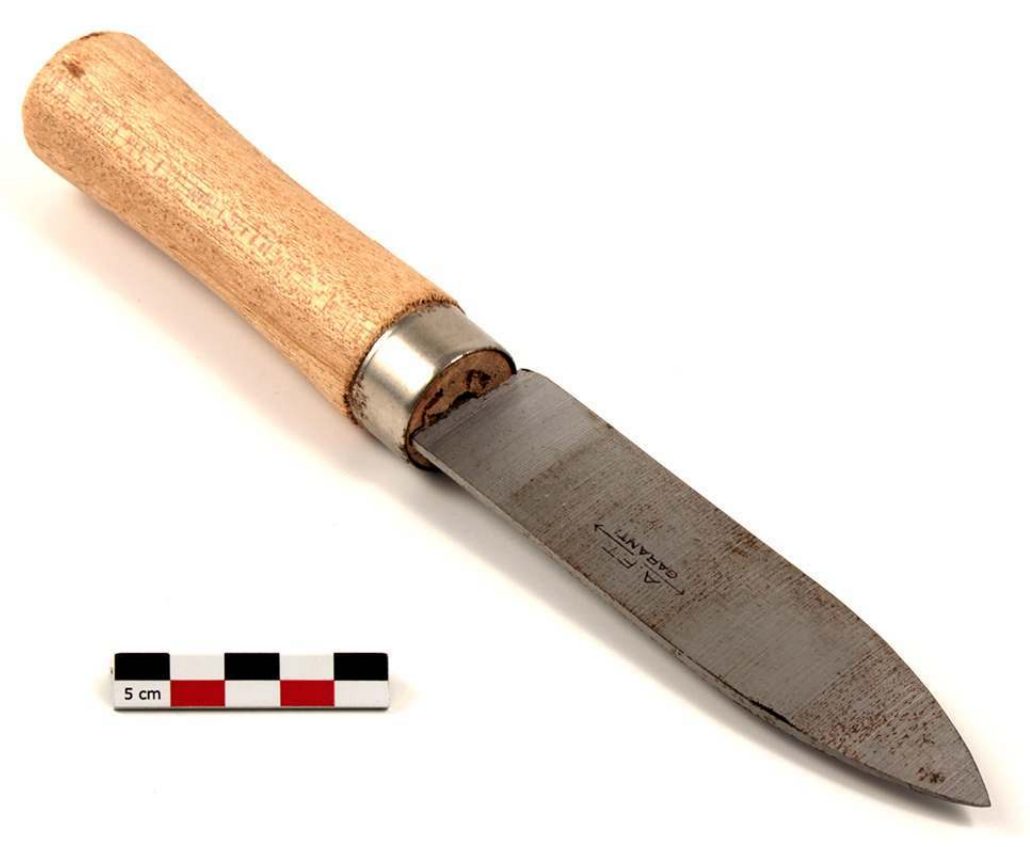

(c) P. Rameau

254 - France ; Fécamp, Seine-Maritime ; achat au Musée des terre-neuvas et de la pêche

- «Couteau Piqueur »; lame à virole. Marque AFT (A. Fourré-Triquet, Sourdeval-la-Barre, Manche). Utilisé lors des campagnes de pêche à Terre-Neuve ou en Islande, ce couteau sert à vider les morues, éventrées d'un seul coup de bas en haut. La fabrique AFT ayant fermé, un stock de couteaux resta disponible au Musée des terre-neuvas et de la pêche

- Années 2000 ; collecteur Richard Dumez

- Longueur $27 \mathrm{~cm}$, largeur $3,5 \mathrm{~cm}$, diamètre du manche $3 \mathrm{~cm}$

- ETB-FR-RD-2018-001

Jean Recher décrit ainsi le travail du piqueur, qui vide la morue avant de la passer au trancheur :

«Attrapant la morue de la main gauche, le piqueur la fiche par la nuque, devant lui à hauteur de sa poitrine, sur un morceau de fer pointu des deux bouts, lui-même enfoncé dans le madrier formant la partie supérieure de la séparation entre le parc des piqueurs et celui des décolleurs. D'un coup de couteau, le marin fend la gorge juste sous les opercules, il ouvre ensuite le ventre jusqu'au cloaque, extrait les viscères qu'il laisse tomber dans la goulotte légèrement inclinée vers l'arrière $d u$ navire et par laquelle circule de l'eau de mer qui entraîne la tripaille vers les ramasseurs de foie. [...]» (Recher $1977:$ 73) 


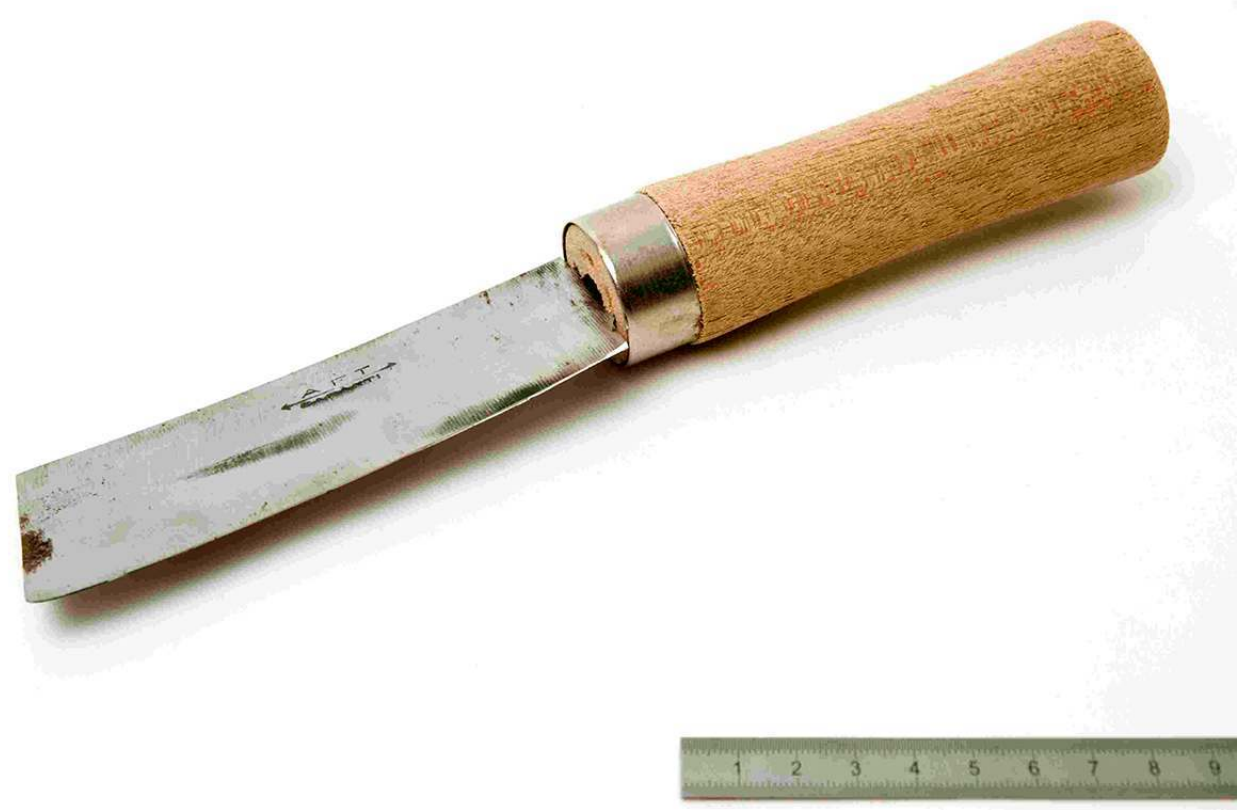

\section{(c) J.-C. Domenech}

- France ; Fécamp, Seine-Maritime ; achat au Musée des terre-neuvas et de la pêche

- Couteau à morue, « Trancheur Grand ». Lame « vrillée » à virole. Marque AFT (A. FourréTriquet, Sourdeval-la-Barre, Manche). Les morues ayant été vidées par un premier matelot, un deuxième fait deux entailles profondes avec le Trancheur, dit aussi Habilleur, le long de l'épine dorsale, qu'il arrache ensuite d'un seul coup. Le corps aplati est ensuite jeté au saleur, dans la calle.

- Années 2000 ; collecteur Richard Dumez

- Longueur totale $25 \mathrm{~cm}$; largeur lame $5 \mathrm{~cm}$, diamètre manche $3,5 \mathrm{~cm}$

- ETB-FR-RD-2018-002

Selon J. Recher, le trancheur reçoit du piqueur la morue vidée et sépare la chair du squelette :

«Le trancheur est l'artiste de la chaîne de travail. [...] Son geste est souple et sûr, son coup de couteau précis et rapide. [...] il transforme le cabillaud en morue ou, pour employer une autre expression, la morue ronde en poisson plat. [...] La saisissant de la main gauche et la plaçant parallèlement au bord de l'étal, d'un coup de couteau partant du chignon et aboutissant à la queue, le trancheur ouvre la morue côté ventre, laissant suffisamment de chair le long de l'épine dorsale pour réunir les deux flancs. Puis retournant son instrument de cent quatre-vingt degrés, il désosse l'arête principale depuis l'endroit où sa section est triangulaire et l'enlève d'un mouvement glissé. La morue, qui n'est plus qu'un triangle présentant un côté peau et un côté chair, tombe dans la baille où elle sera lavée et l'arête (le nôs) reste dans la main du marin qui la jette sur le pont et du même mouvement tournant du bras se saisit de la morue suivante. » (Recher 1977 : 74-75) 
Figure 130 : Prospectus (recto et verso) de la coutellerie AFT

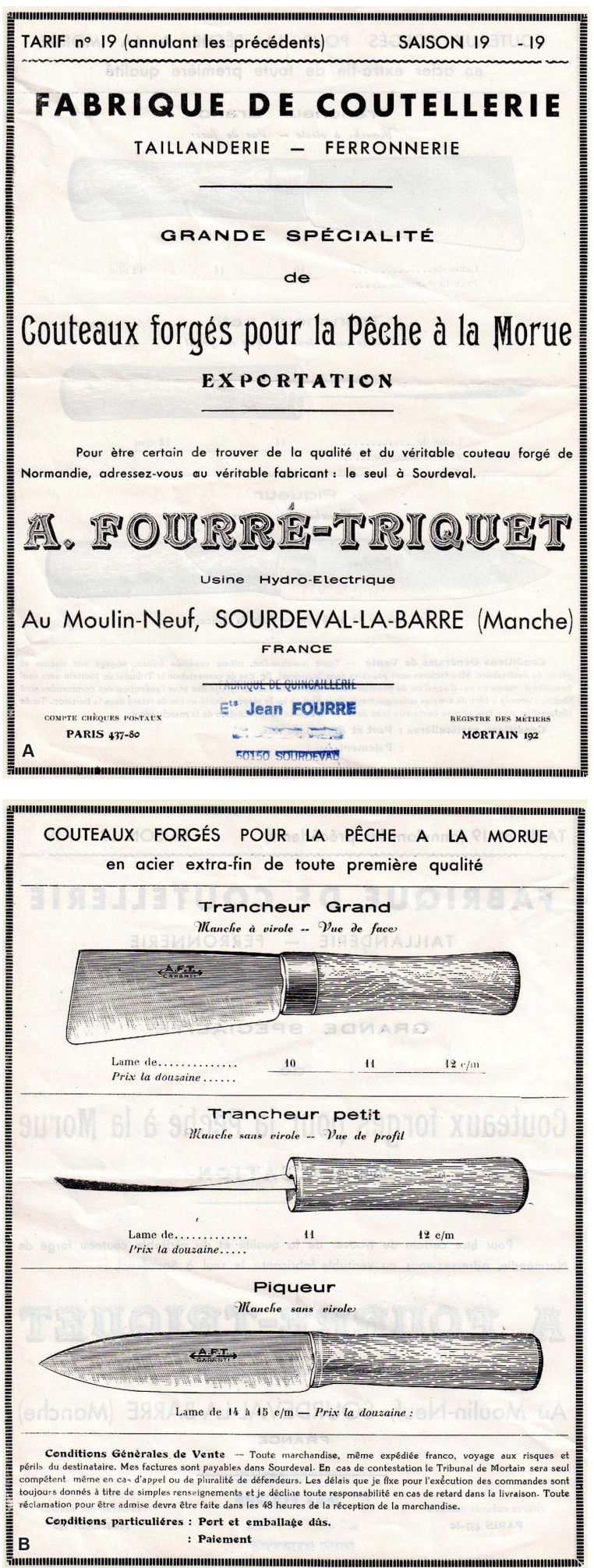


Figure 131 : L'organisation de la préparation des morues expliquée dans Duhamel du Monceau. A) ustensiles nécessaires à la pêche. B) Appareillage du navire

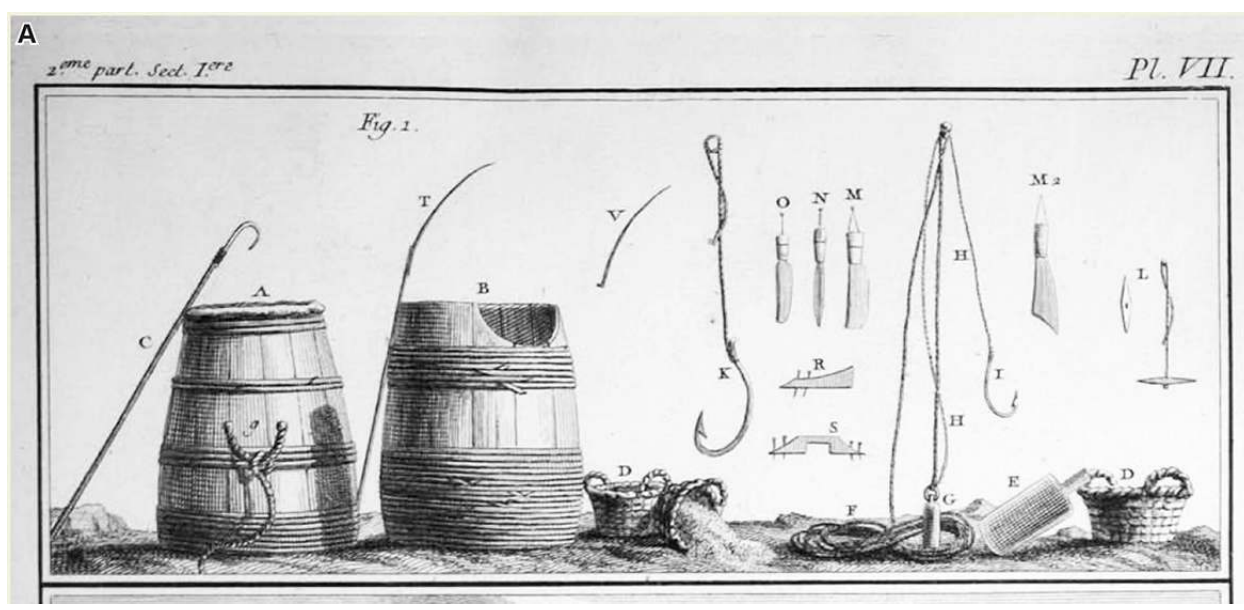

Vol. 2, planche VII, figure 1 : « Une partie des ustensiles nécessaires pour faire la pêche de la Morue sur le grand Banc ».

$A$ : baril dans lequel se mettent les pêcheurs : $B$ : baril qu'on met auprès des habilleurs pour y mettre les foies ; C : gaffe qui sert aux habilleurs pour attirer les morues ; D : panier pour transporter «les breuilles, les langues, les foies, le sel, \&c. »; E : petite pelle pour le service des saleurs dans la calle; K : hain (hameçon)F, G, H, I : ligne, hain et plomb; $T$ : piquoir pour pousser les morues vers les habilleurs. " M, PI. VII, est une espèce de couperet qu'on nomme Couteau à Habiller. Chaque Habilleur doit avoir le sien : assez souvent ce couteau est fait comme M2. N, PI. VII, est un couteau à deux tranchants, qui sert à emporter les têtes, \& qu'on nomme pour cette raison, Etêteur.

$\mathrm{O}, \mathrm{PI}$. VII, autre couteau nommé Nautier, parce qu'il sert à détacher les noues². »

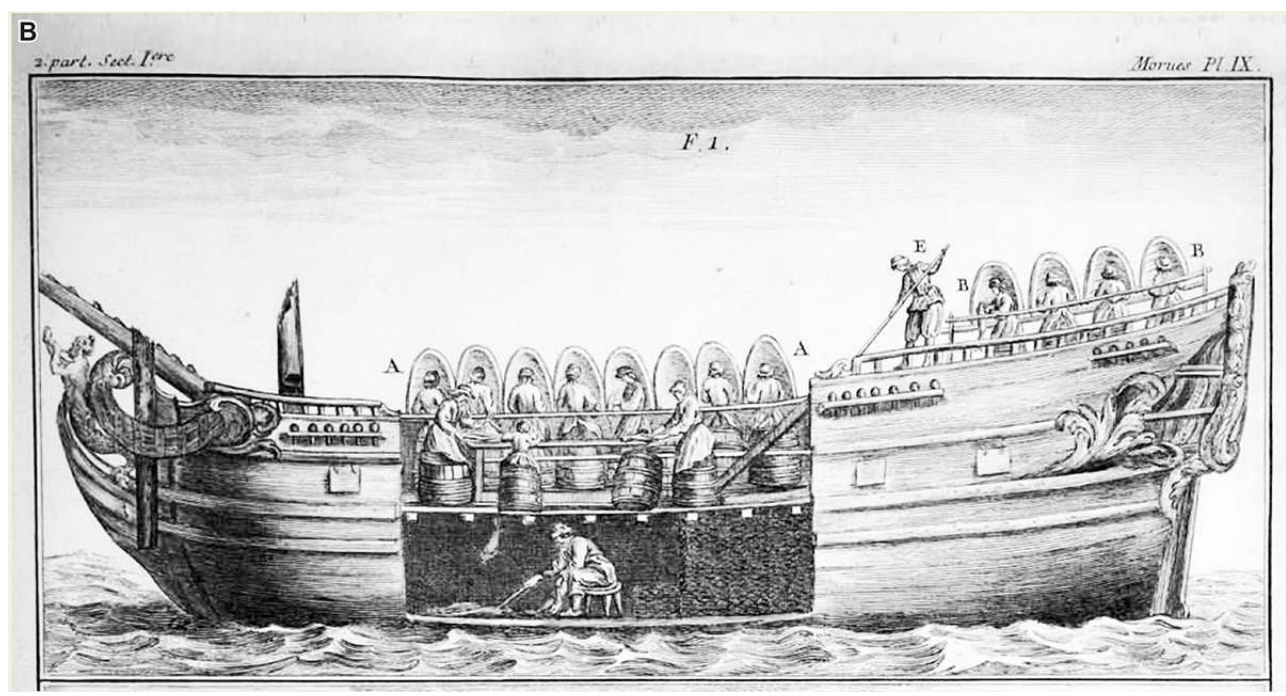

Planche IX, figure 1 : Bâtiment appareillé suivant l'usage de Normandie pour la pêche de la Morue sur les Bancs. A et B : les pêcheurs (ligneurs), protégés du vent par des niches, le theu. $\mathrm{E}$ : garçon de bord qui pousse les poissons pris par les pêcheurs vers la table de travail. Au centre du bateau : l'étal: ... « une table de bois (...) à un des bouts de laquelle est un Décoleur, \& à l'autre un Habilleur, chacun dans son barril ; au milieu est aussi dans son barril un Mousse, qu'on nomme Nautier, parce que sa fonction est de détacher les noues ou nauts. On voit encore un barril incliné (...) destiné à recevoir les foies ; dans la cale est un Saleur avec des tas de Morues salées en grenier. » 


\section{Pour conclure}

\section{Symbolique}

\section{4) Les pez-diablo du Mexique}

Figure 132 : Raies sèches PEZ DIABLO ETB-MX-SB-2010A-012 recto et verso, et détail de la tête " diabolique »

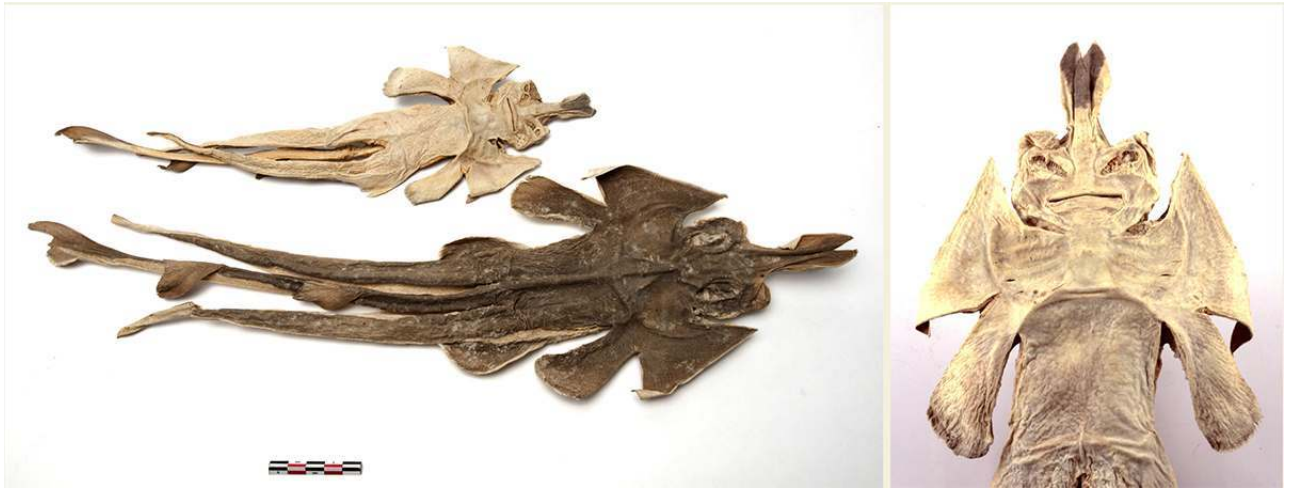

(C) J.C. Domenech et S. Bahuchet

260 - Mexique ; Guadalajara, État du Jalisco ; acheté au marché Corona

- Pez diablo " poisson-diable ». Deux raies (Squatinidés) sèches et préparées de façon à évoquer une représentation du diable, sur la face ventrale. Se suspend dans la maison, comme charme de protection

- 09/04/2010 ; collecteur Serge Bahuchet

- La plus grande : $34 \mathrm{~cm}$ de longueur

- ETB-MX-SB-2010A-012

Représentation artistique

95) Tableau de Bass «Pêcheurs lebous» 


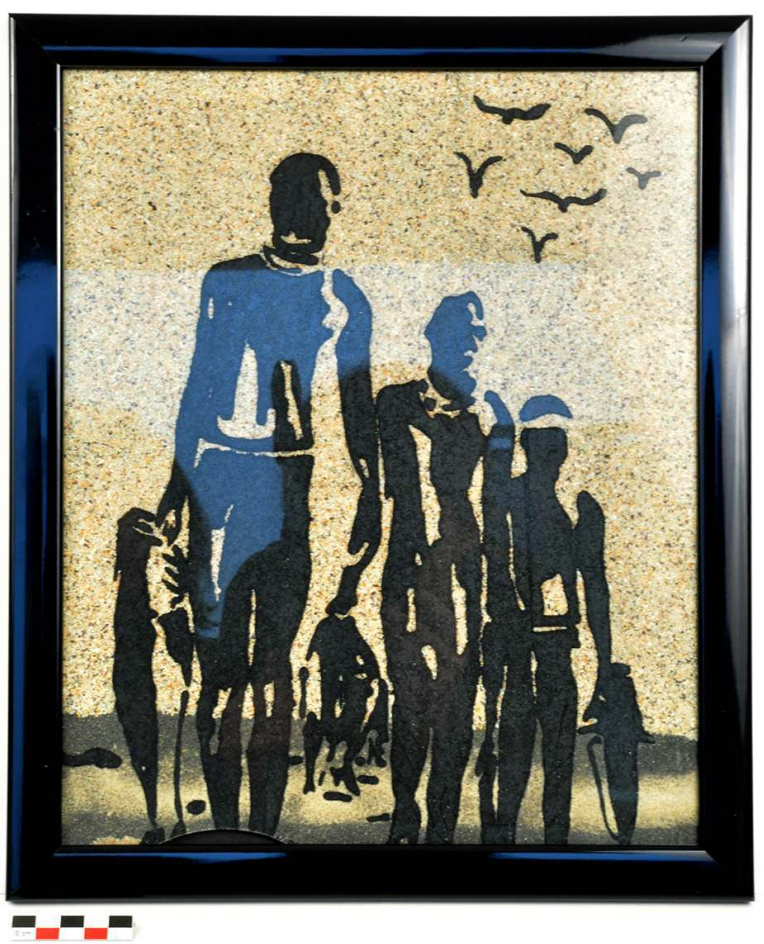

(c) D. Brabant

- Sénégal ; île de Gorée, Dakar

- Tableau dit «Pêcheurs lébous» par Bassirou Sidy N'Diaye dit «Bass ». Fixé sous verre, dessin à l'encre de Chine, sur fond de sable coquillier aux grains de différentes couleurs (noir, beige, rose et blanc). Trois pêcheurs se trouvent au centre du tableau, chacun dispose d'un ou plusieurs poissons tenus dans leurs mains. En haut à droite de l'œuvre y figure un vol d'oiseaux. A l'arrière-plan, on aperçoit la mer

- 1999 ; donné par Monique Camus

$\cdot 38 \times 32 \mathrm{~cm}$

- MNHN-E-2017.12.2

\section{Liste des objets par pays}

Algérie

Dessin d'enfant : MNHN-E-2013.8.136(2)

Bénin

Panier-cloche : MNHN-E-2009.19.101

Brésil

Épuisette : ETB-BR-SB-2009-010

Filet : ETB-BR-MC-2013-001

Flèche-harpon : MNHN-E-2012.3.19

Foënes : ETB-BR-MC-2013-005, ETB-BR-SB-2009-005

Nasse : ETB-BR-PL-2014-001 
266

268

269

270

Chine

Jouet : MNHN-E-2011.9.52

Croatie

Foëne : ETB-HR-VB-2016-001

Équateur

Filet-épuisette : ETB-EC-GZB-2012-005

Espagne

Turlutte : ETB-ES-FB-2018-001

France

Calibreurs (mesures) : ETB-FR-SB-2016-001, ETB-FR-SB-2016-002

Coupelles pour les huîtres : ETB-FR-SB-2018-006

Couteau à huîtres : ETB-FR-CDNR-2012-001, ETB-FR-CDNR-2012-002

Couteaux à morues : ETB-FR-RD-2018-001, ETB-FR-RD-2018-002

Crochet pour crabe : ETB-FR-RP-2011-046

Épervier : ETB-FR-RP-2011-072

Flotteurs : ETB-FR-RP-2012-040, ETB-FR-RP-2012-041, ETB-FR-RP-2012-055

Flotteurs : ETB-FR-SB-2009-004, ETB-FR-SB-2018-004, ETB-FR-SB-2018-005

Foënes : ETB-FR-RP-2011-069, ETB-FR-RP-2011-070, ETB-FR-RP-2011-071

Grattoir-épuisette : ETB-FR-RP-2011-052

Hameçons : ETB-FR-RP-2012-056, ETB-FR-SB-2016-003, ETB-FR-MC-2018-001

Mouches : ETB-FR-NR-2015-001, ETB-FR-NR-2015-002

Nasses : ETB-FR-RP-2011-073, ETB-FR-RP-2011-074

Paniers à coquillages : ETB-FR-JLD-2016-001 ETB-FR-SLB-2016-001

Pot à poulpe : ETB-FR-SB-2018-005

Sac à poisson sur cadre : ETB-FR-RP-2011-004

Trousse de 10 turluttes : ETB-FR-SB-2018-003

Turlutte-cage et appât : ETB-FR-SB-2018-001et 002

Verveux : ETB-FR-NR-2018-001

Groenland

Embarcation : MNHN-E-2016.6.87

Filets : MNHN-E-2016.6.80, MNHN-E-2016.6.81

Hameçons : MNHN-E-2016.6.23, MNHN-E-2016.6.89

Guyane française

Arc : ETB-GF-HP-2000-005

Épuisette : ETB-GF-HP-2000-008

Flèches : ETB-GF-HP-2000-001, ETB-GF-HP-2000-002, ETB-GF-HP-2000-003

Nasses : ETB-GF-SB-2014-15, ETB-GF-SB-2014-42

Pique à main : ETB-GF-HP-2000-007

273

donésie

Pagaies : ETB-IN-ALB-2013-001, ETB-IN-ALB-2013-002

274 Italie

Filet tellinier : ETB-IT-NR-2015-004

Nasses : ETB-IT-NR-2015-001, ETB-IT-NR-2015-002

Palangre : ETB-IT-NR-2015-003 
275 Japon

Étendards d'embarcation : ETB-JP-NC-2013-01, ETB-JP-NC-2013-02, ETB-JP-NC-2013-03, ETB-JP-NC-2013-04

Hameçon : ETB-JP-SB-2018-002

276 Laos

Panier à poissons : ETB-LA-FA-2017-015

277 Libéria

Crochet pour ravauder les filets : MNHN-E-2013.1.7

Filet : MNHN-E-2012.6.14

Nasses : MNHN-E-2012.6.13, MNHN-E-2014.9.5

278 Mexique

Hameçons : MNHN-E-2002.2.153, ETB-MX-FA-2009-12

Leurre : ETB-MX-FA-2009-010

Raies sèches pez diablo : ETB-MX-SB-2010A-012

279 Papouasie-Nouvelle Guinée

Haveneau : MNHN-E-2006.1.28

Pagaie : MNHN-E-2006.1.35

Nasse : MNHN-E-2012.1.8

280 Pérou

Embarcations : MNHN-E-2010.1.1, ETB-PE-MQ-2009-008, ETB-PE-MQ-2009-023

Filets : ETB-PE-MQ-2009-027, ETB-PE-MQ-2009-028

Hameçons : MNHN-E-2001.40.403, MNHN-E-2001.40.299, ETB-PE-MQ-2009-030, ETB-PEMQ-2009-044

Harpon : MNHN-E-2001.40.278

Ligne avec son plioir : ETB-PE-MQ-2009-029

Pagaie : ETB-PR-ERO-2017-009

Piège à crabe : ETB-PE-MQ-2009-026

Représentation de la pêche : ETB-PE-MQ-2009-009

281 République dominicaine

Massue de pêche : MNHN-E-2010.3.1

282 Salomon (Iles)

Appeau à requins : MNHN-E-2002.15.19

Hameçon : ETB-NC-FA-2018-001

Pagaie : MNHN-E-2009.15.6, MNHN-E-2009.15.6 7

283 Sénégal

Tableau sous verre : MNHN-E-2017.12.2

284 Surinam

Pagaie : ETB-SR-MCC-2003-032

285 Tunisie

Nasse : MNHN-E-2014.17.8

286 Vanuatu

Embarcation : MNHN-E-2012.8.1

Hameçon : ETB-VU-FB-2006-001

287 Venezuela

Embarcation : MNHN-E-2012.7.6 
Flèches : MNHN-E-2012.7.11(2,3), MNHN-E-2012.7.13(3)

Foëne : MNHN-E-2012.7.13(4)

Ligne flottante : MNHN-E-2012.7.7

Vietnam

Nasses : ETB-VN-SBF-2016-001, ETB-VN-SBF-2016-002

Paniers à poissons : ETB-VN-ALB-2009-003(a, b)

Sans origine

Panier de capture : MNHN-E-2009.30.85

Les auteurs remercient Myriam Kourdourli, technicienne des collections d'ethnologie du Muséum, pour leur avoir communiqué les fiches techniques et les photographies des objets de celles-ci. Ils remercient également les collecteurs qui leur ont aimablement confié des photographies prises sur leurs terrains: Ariadna Burgos, Pauline Laval, Marina Quiñe, Emmanuelle Ricaud Oneto, Pierre Robbe, Pascale de Robert, Gabriela Zurita et tHélène Pagezy.

\section{BIBLIOGRAPHIE}

Bahuchet S., Rameau P. 2016 - Quelques engins de pêche en eau douce d'Afrique centrale. Revue d'ethnoécologie 10. URL : http://ethnoecologie.revues.org/2877

Banack S. A., Rondón X. J., Diaz-Huamanchumo W. 2004 - Indigenous Cultivation and Conservation of Totora (Schoenoplectus californicus, Cyperaceae) in Peru. Economic botany 58 (1) : 11-20.

Bataille-Benguigui M.-C. 1981 - La capture au requin du nœud coulant aux îles Tonga : persistance et changements dans l'observation des interdits. Journal de la Société des océanistes 37 (72-73) : 239-250.

Burgos A. L. 2013 - Ethnoécologie d'une société mentawai : femmes, mangroves et coquillages de l'île de Siberut (Indonésie). Thèse, Paris, MNHN, 502 p.

Claridge G.F., Sorangkhoun T., Baird I.G. 1997 - Community fisheries in Lao PDR: a survey of techniques and issues. Vientiane, IUCN, $70 \mathrm{p}$

Davy D. 2007 - «Vannerie et vanniers »; approche ethnologique d'une activité artisanale en Guyane française. Thèse, Université d'Orléans, $526 \mathrm{p}$.

Duhamel du Monceau H. L. 1769-1772 - Traité général des pesches et histoire des poissons qu'elles fournissent. Paris, Saillant \& Nyon

Fouchier D., Chevalier J.-P. 1973 - L'anguille dans le marais poitevin. Science et Nature.

L'environnement 120 : 15-26.

Grenand P. 1995 - De l'arc au fusil. Un changement technologique chez les Wayãpi de Guyane. In : Grenand F., Randa V. (Ed.) Transitions plurielles, exemples dans quelques sociétés des Amériques. Paris, Peeters/Selaf : 23-53

Hérisset R. 2014 - La vannerie en Bretagne. Presses de l'Université de Rennes, 408 p. 
Hurault J.-M., Grenand F., Grenand P. 1998 - Indiens de Guyane. Wayana et Wayampi de la forêt. Paris, Autrement, $200 \mathrm{p}$.

Laval P. 2016 - Captures estuariennes. Une ethnoécologie de la pêche sur le bas Oyapock (frontière francobrésilienne). Thèse, Paris, MNHN, $532 \mathrm{p}$.

Lecointre G., Le Guyader H. 2001 - Classification phylogénétique du vivant. Paris, Belin, 559 p.

Lecuyer J., Pujol R. 1975 - L'oie plumassière du Poitou, utilisation des peaux et des plumes. In :

L'homme et l'animal, premier colloque d'ethnozoologie. Paris, Institut international d'ethnosciences : 205-216.

Leroi-Gourhan A. 1973 [1945] - Milieu et techniques. Paris, Albin Michel, 475 p.

Meunier F. J. (éd.) 2004 - Piranhas enivrés, des poissons et des hommes en Guyane. Paris, SFI/RMN, $128 \mathrm{p}$.

Monod T. 1973 - Contribution à l'établissement d'une classification fonctionnelle des engins de pêche. Bulletin du Muséum national d'Histoire naturelle, écologie générale, 156 (12) : 205-231.

Moretti C. \& Grenand P. 1982 - Les nivrées ou plantes ichtyotoxiques de la Guyane française. Journal of Ethnopharmacology 6 : 139-160.

Pagezy H., Jégu M. 2004 - Le contexte de la pêche : techniques et saisons. In : Meunier (Ed.) Piranhas envivrés : 43-54.

Pagezy H., Jégu M. 2010 - Guyane française. Les indiens Wayana du Haut Maroni. In : Pagezy H., Carrière S., Sabinot C. (Ed.) Nature du monde. Dessins d'enfants. Paris, CTHS : 148-163.

Quiñe Fernandez M. 2009 - Sur le dos d'un « caballito de totora » : étude d'une pratique de pêche artisanale et millénaire au futur incertain. Huanchaco, la Libertad, Pérou. Mémoire de Master 2, EDTSMNHN, $79 \mathrm{p}$.

Recher J. 1977 - Le grand métier. Paris, Plon, 497 p.

Reyes N. 2016 - Aux confins des mesures internationales, une gestion locale. Itinéraire des pêcheurs d'anguilles en Méditerranée. In : Robert S., Melin H. (Ed.) Habiter le littoral ; enjeux contemporains. Aix-en-Provence, Presses universitaire de Provence : 257-274.

Reyes N. 2017 - À l'aune des politiques internationales et européennes. Pratiques des pêcheurs méditerranéens et systèmes de gestion communautaire. Catalogne-Occitanie-Sicile. Thèse, Paris, MNHN, $442 \mathrm{p}$.

Robbe B. 2010 - Les Tunumiit de la côte Est du Groenland. In : Pagezy H., Carrière S., Sabinot C. (Ed.) Nature du monde. Dessins d'enfants. Paris, CTHS : 22-43.

Robbe P. 1994 - Les Inuit d'Ammassalik, chasseurs de l'Arctique. Paris, Éditions du Muséum national d'Histoire naturelle, $390 \mathrm{p}$.

Romdhane M. S. 1998 - La pêche artisanale en Tunisie. Évolution des techniques ancestrales. Mélanges de l'École française de Rome. Antiquité 110 (1) : 61-80.

Rondelet G. (1558) 2002 - L'histoire entière des poissons. Paris, CTHS, 181 p.

Rondón X. J., Banack S. A., Diaz-Huamanchumo W. 2003 - Ethnobotanical investigation of caballitos (Schoenoplectus californicus: Cyperaceae) in Huanchaco, Peru. Economic Botany, 57 (1) : 35-47.

Suarez M. M. 1966 - Les utilisations du palmier "Moriche" (Mauritia flexuosa L. f.) chez les Warao du delta de l'Orénoque (territoire delta Amacuro, Venezuela). Journal d'agriculture tropicale et de botanique appliquée 13 (1-3) : 33-38. 
Suarez M. M. 1968 - Los Warao: indígenas del delta del Orinoco. Caracas, Departamento de Antropologia, Instituto Venezolano de Investigaciones Cientificas, $311 \mathrm{p}$.

Vuković I. 2014 - Man and the sea: fishing. Fishing items from the collection of the Split Ethnographic Museum. Etnografski Muzej Split 198 p.

Zurita Benavides M. G. 2014 - Du «temps du tapir » à nos jours : les marques du temps dans le paysage. Perspectives de deux villages waorani sur les relations entre les espaces forestiers et le temps en Amazonie équatorienne. Thèse, Paris, MNHN, 365 p.

\section{NOTES}

1. L'ensemble d'ethnologie est issu de l'ancien laboratoire d'ethnologie du MNHN et résulte des activités de ses chercheurs et de dons d'amateurs. Il comporte environ 4000 objets, dont une partie est inscrite au patrimoine au terme de la loi relative aux Musées de France. L'ensemble d'ethnobiologie résulte des recherches de l'ancien laboratoire d'ethnobiologie-biogéographie, puis de celles de l'Unité mixte de recherche éco- anthropologie et ethnobiologie (UMR 7206). Il s'agit d'une "collection d'étude", au sens du règlement des collections du MNHN, d'environ 3800 objets.

2. Noues, nauts : les vessies natatoires.

\section{RÉSUMÉS}

Nous décrivons 95 objets ou lots d'objets se rapportant aux techniques de pêche, conservés dans les collections d'anthropologie culturelle du Muséum national d'histoire naturelle, au Musée de l'Homme : 77 engins de pêche, et des objets accessoires (liés aux embarcations, à la manipulation des prises ou à leur préparation après la capture), recueillis dans 26 pays différents. Cet ensemble est présenté selon la classification fonctionnelle élaborée par Théodore Monod (1973), que nous avons modifiée pour les pêches avec des hameçons. Nous pouvons ainsi illustrer et commenter 17 techniques de pêche différentes. Ce catalogue complète celui publié en 2016 et consacré à 22 objets d'Afrique centrale.

\section{AUTEURS}

\section{SERGE BAHUCHET}

Professeur d'ethnobiologie du Muséum, laboratoire Eco-anthropologie et ethnobiologie et responsable scientifique des collections d'anthropologie biologique et culturelle (MNHN-CNRSParis Diderot)

\section{PAULINE RAMEAU}

Chercheur associée et assistante de gestion des collections d'ethnobiologie, laboratoire Ecoanthropologie et ethnobiologie (MNHN-CNRS-Paris Diderot) 


\section{NASTASSIA REYES}

Chercheure en anthropologie, UMR 7206 Éco-anthropologie et Ethnobiologie (CNRS-MNHN), UMR MARBEC (IRD-IFREMER-UM2-CNRS)

Reyes.nastassia@gmail.com 